\title{
Comparative Analysis of Uninhibited and Constrained Avian Wing Aerodynamics
}

Jordan A. Cox

Follow this and additional works at: https://researchrepository.wvu.edu/etd

\section{Recommended Citation}

Cox, Jordan A., "Comparative Analysis of Uninhibited and Constrained Avian Wing Aerodynamics" (2016). Graduate Theses, Dissertations, and Problem Reports. 5404.

https://researchrepository.wvu.edu/etd/5404

This Thesis is protected by copyright and/or related rights. It has been brought to you by the The Research Repository @ WVU with permission from the rights-holder(s). You are free to use this Thesis in any way that is permitted by the copyright and related rights legislation that applies to your use. For other uses you must obtain permission from the rights-holder(s) directly, unless additional rights are indicated by a Creative Commons license in the record and/ or on the work itself. This Thesis has been accepted for inclusion in WVU Graduate Theses, Dissertations, and Problem Reports collection by an authorized administrator of The Research Repository @ WVU. For more information, please contact researchrepository@mail.wvu.edu. 


\title{
Comparative Analysis of Uninhibited and Constrained Avian Wing Aerodynamics
}

\author{
Jordan A. Cox
}
Thesis submitted to the
College of Engineering and Mineral Resources
at West Virginia University in partial fulfillment of the requirements
for the degree of

\author{
Master of Science \\ in \\ Aerospace Engineering \\ Patrick Browning, Ph.D., Chair \\ Wade Huebsch, Ph.D. \\ Peter Gall, Ph.D. \\ Yogendra Panta, Ph.D.
}

Department of Mechanical and Aerospace Engineering

Morgantown, West Virginia

2016

Keywords: Biometrics, Avian, Wing, Aerodynamics, Experimental

Copyright 2016 Jordan Cox 


\section{ABSTRACT \\ Comparative Analysis of Uninhibited and Constrained Avian Wing Aerodynamics}

\section{Jordan A. Cox}

The flight of birds has intrigued and motivated man for many years. Bird flight served as the primary inspiration of flying machines developed by Leonardo Da Vinci, Otto Lilienthal, and even the Wright brothers. Avian flight has once again drawn the attention of the scientific community as unmanned aerial vehicles (UAV) are not only becoming more popular, but smaller. Birds are once again influencing the designs of aircraft. Small UAVs operating within flight conditions and low Reynolds numbers common to birds are not yet capable of the high levels of control and agility that birds display with ease. Many researchers believe the potential to improve small UAV performance can be obtained by applying features common to birds such as feathers and flapping flight to small UAVs. Although the effects of feathers on a wing have received some attention, the effects of localized transient feather motion and surface geometry on the flight performance of a wing have been largely overlooked. In this research, the effects of freely moving feathers on a preserved red tailed hawk wing were studied. A series of experiments were conducted to measure the aerodynamic forces on a hawk wing with varying levels of feather movement permitted. Angle of attack and air speed were varied within the natural flight envelope of the hawk. Subsequent identical tests were performed with the feather motion constrained through the use of externally-applied surface treatments. Additional tests involved the study of an absolutely fixed geometry mold-and-cast wing model of the original bird wing. Final tests were also performed after applying surface coatings to the cast wing. High speed videos taken during tests revealed the extent of the feather movement between wing models. Images of the microscopic surface structure of each wing model were analyzed to establish variations in surface geometry between models. Recorded aerodynamic forces were then compared to the known feather motion and surface geometry to correlate the performance to these two features. The results of this study revealed that the performance of the bird wing was directly affected by feather motion. It was also found that the motion of covert and secondary covert feathers had the greatest influence on the performance. Increased coefficients of lift and drag were found when higher frequencies of these feathers were observed. Noticeable reductions in the coefficient of drag were found to be associated with micron level variations in the depth of surface features on the wing. 


\section{Dedication}

While I performed the hours of work and research required to complete this thesis, I kept who it should be dedicated to. By looking at theses written by those before me, I noticed most were dedicated to just one person, usually the authors' mother, a relative, or a mentor who had served as an influential part of their life.

As I continued to perform hours of the often tedious work, I realized, no one would ever see the amount of behind the scenes work I had conducted on late nights after work or classes, those terrible Mondays after drill, and the good times I missed during my education with friends and co-workers who would eventually fade from my life. It was then that I realized I couldn't dedicate this work to just one person. My life's accomplishments (to include this thesis) and who I have grown to become are not the results of just one person or even a small group of people. Like this thesis, all these things are the results of the extra efforts people have invested in me that no one ever saw.

In a way these people's efforts were the same as all those late night and extra sessions I put into this work. Performing these acts on just one occasion has a minimal impact on the big picture. The message $I$ have been building up to deliver is, no one act of kindness to educate or motivate is enough. Great things are the results of continuous work on one project or one person over period of time.

Finally, I would like to dedicate this thesis to the following individuals and groups:

- My mother, Pam, who has by far invested the most in me and provided constant support and encouragement when I needed it the most.

-My father, Andrew, who provided the best example possible of how a man should live his life and how an officer should act, but how to treat those around you with respect.

-All of my former and current companions in the Air National Guard, Kevin Wolfe, Leonard Dyer, and Mathew Ford, who helped me to better understand the importance of not only hard work, but precise work and the patience required to be successful.

-My friends at 4D Tech Solutions, Bradley DeRoos and Kiril Speransky, thank you for your patience and for mentoring me in realm of design, development, and testing. My time working with you and the rest of the 4D team has been the best part of my experience in Morgantown. 


\section{Acknowledgements}

I would like to thank the West Virginia Space Grant Consortium (WVSGC) for their support. By awarding me with a graduate fellowship, the WVSGC made it possible for me to purchase the materials needed for this research and allowed me the financial freedom to devote the required time to this project. Furthermore, I would like to thank Mrs. Candy Cordwell for all of her help during my graduate studies. It was through her willingness to help that I became aware of the opportunities that the WVSGC offered.

I would also like to thank my research advisor, Dr. Patrick Browning, for his guidance during my graduate studies. Dr. Browning was always willing to provide the support needed to keep my project on track. Together, we spent many late nights and university holidays when no other professor was in sight, collecting data, building models, or working out what the next step for my project should be. By answering several late night phone calls and quickly responding emails late at night and on weekends, Dr. Browning helped me to keep this project moving. 


\section{Table of Contents}

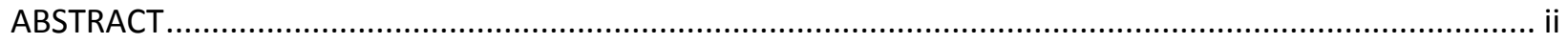

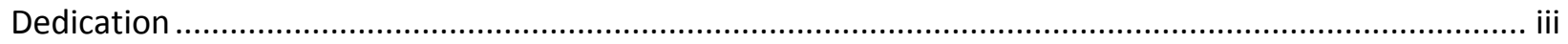

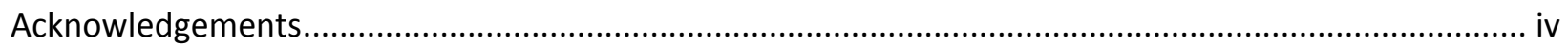

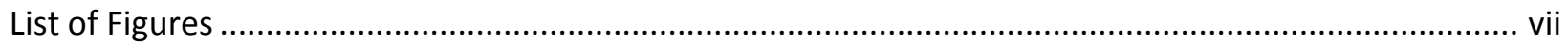

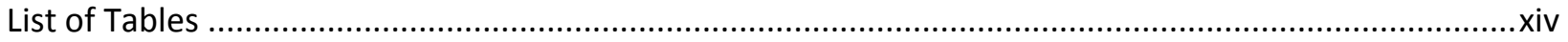

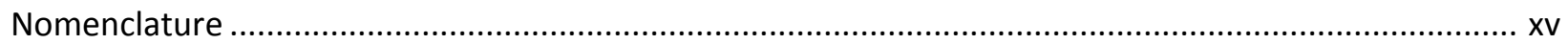

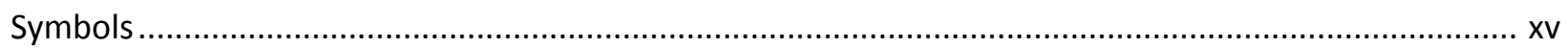

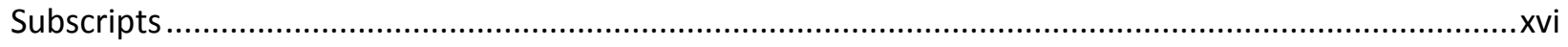

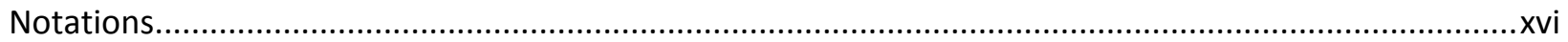

Chapter 1: Problem Statement and Research Objectives................................................................. 1

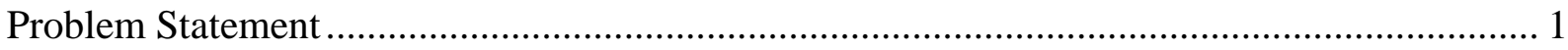

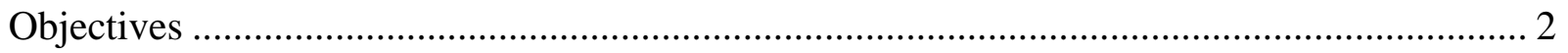

Chapter 2: Review of Relevant Literature.......................................................................................... 3

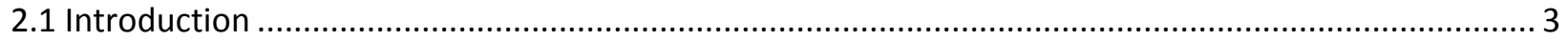

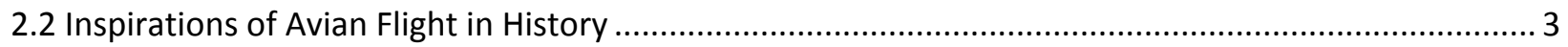

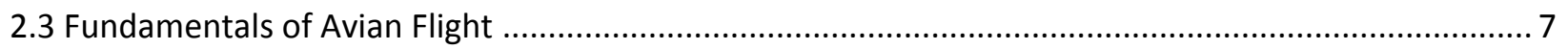

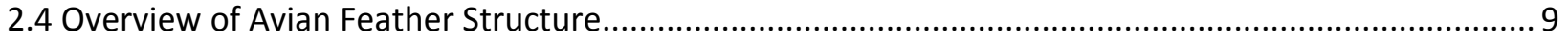

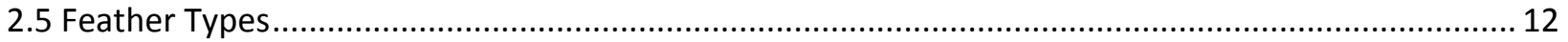

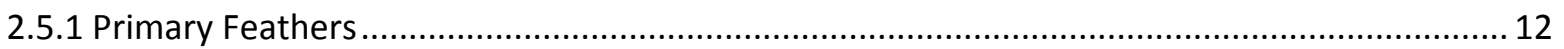

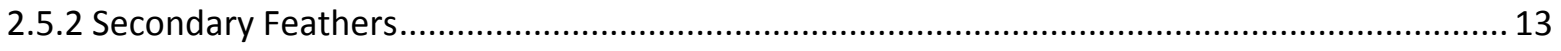

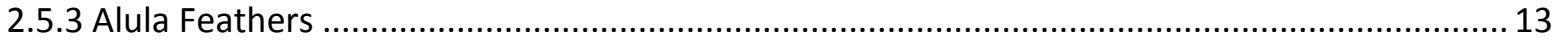

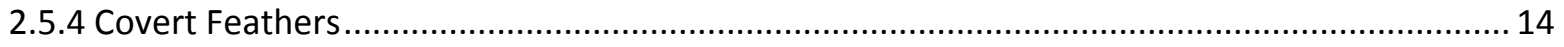

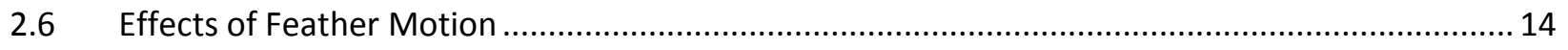

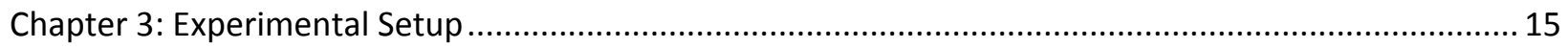

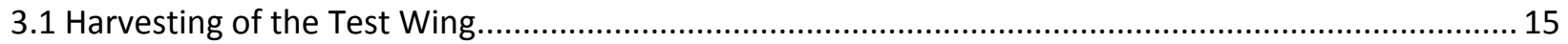

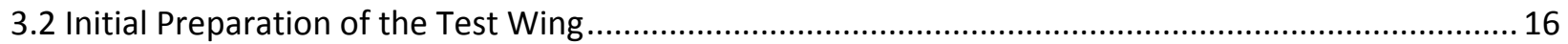

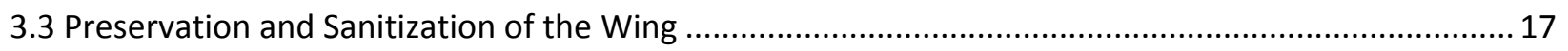

3.4 Surface Applications to Reduce Feather Motion ............................................................................. 21

3.5 Replication of the Natural Wing through a Mold and Cast Process ...................................................24

3.5.1 Development of the Mold and Cast Process................................................................................ 24 
3.5.2 Replication of a Wing Using the Developed Mold and Cast Process ....................................... 30

3.5.3 Preparation of the Cast Wing Model for Testing ….............................................................. 38

3.6 Application to Reduce Surface Geometry of the Cast Wing .......................................................... 41

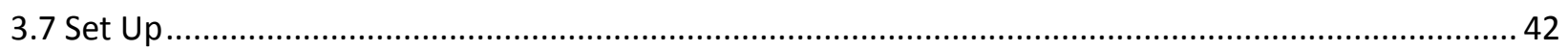

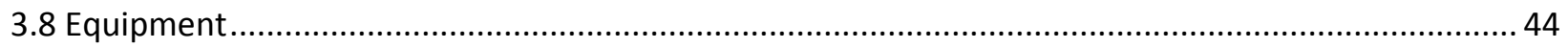

3.8.1 WVU Low Speed Closed-Loop Wind Tunnel ........................................................................... 44

3.8.2 AeroLab Six-Component Internal Strain Gage Force and Moment Balance............................. 45

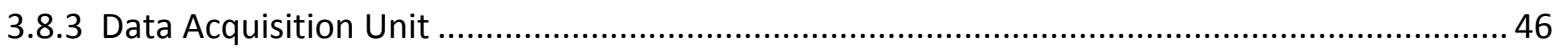

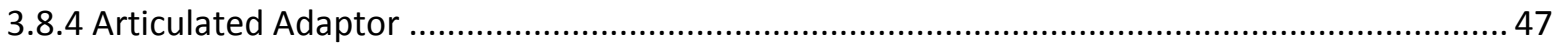

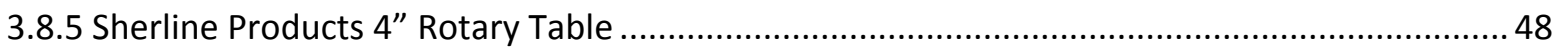

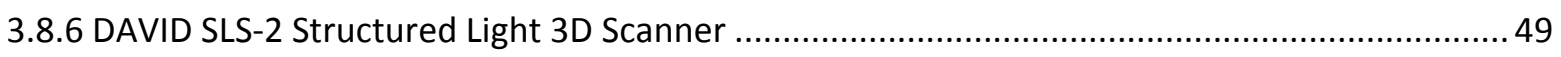

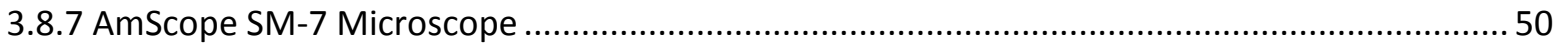

Chapter 4: Method

4.1 Calculation the Mean Chord Length, Area, and Aspect Ratio of the Wing Models............................51

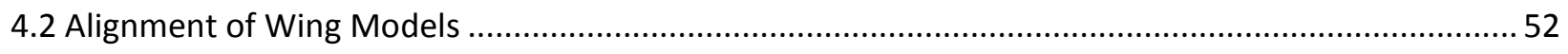

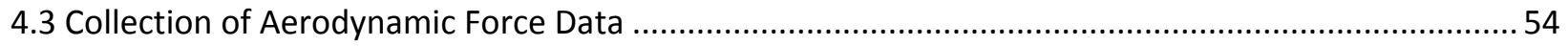

4.4 Comparison of the Macroscopic Shape of the Cast and Biologic Wing Models ................................. 55

4.5 Evaluation of Surface Geometry Differences Across the Wing Models............................................. 56

4.6 Finding the Feather Frequency and Amplitude of Wing Models ...................................................5 57

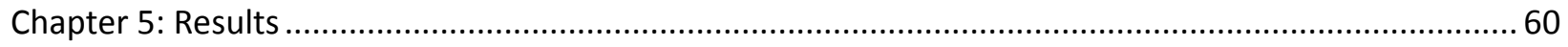

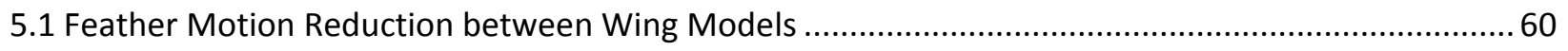

5.2 Surface Geometry Variations Resulting from Model Preparations ...................................................6 68

5.3 Influence of Treatment on Feather Material Properties ................................................................. 75

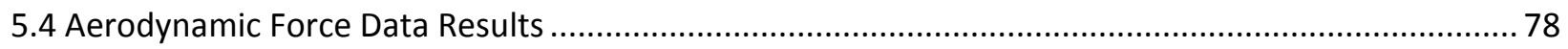

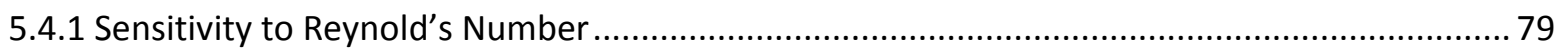

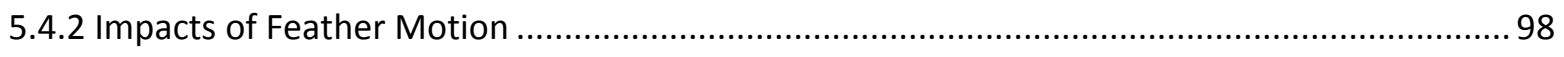

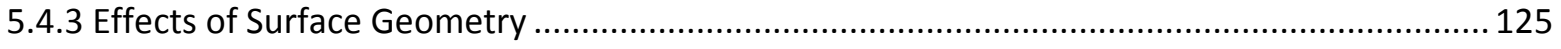

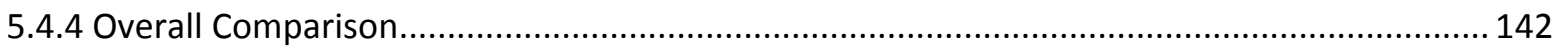

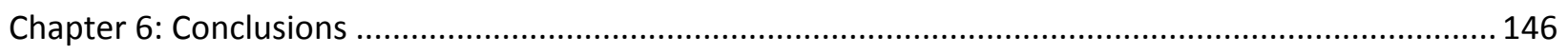

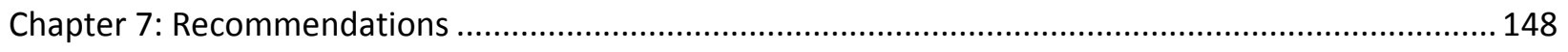

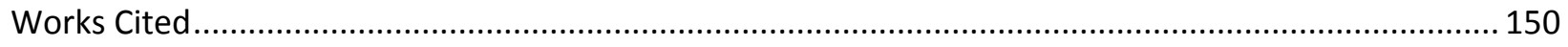

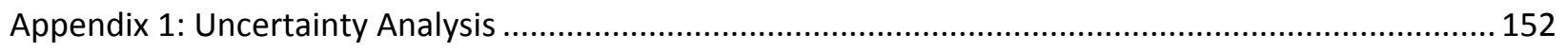




\section{List of Figures}

Figure 1: A Sketch of the Da Vinci Flying Machine [6]

Figure 2: One of Lilienthal's Final Glider Designs Inspired by Birds [2] ___ 5

Figure 3: A Model of the 1903 Wright Flyer at the Smithsonian National Air and Space Museum [9] ___ 6

Figure 4: The Major Wing Regions as Seen on a Red Tailed Hawk Wing [13] ___ 8

Figure 5: The Major Feather Structures as Seen on the Feather of a Finch ___ 9

Figure 6: The Barbs of a Red Tailed Hawk Feather's Vane as Seen Under a Microscope ___ 11

Figure 7: The Major Feather Groups of a Bird [15] ___ 12

Figure 8: The Aluminum Mounting Fixture Attached to the Base of the Wing for Testing___ 16

Figure 9: A Red Tailed Hawk Wing Fix to Wire Mesh with Fishing Line Restraints to Control the Wing Shape __ 18

Figure 10: A Wing Covered in the Descant Mixture during the Preservation Process ___ 19

Figure 11: An Image of the Rigid and Sanitary Wing Resulting from the Preservation Process___ 20

Figure 12: Hair Spray being applied to the Preserved Wing without Disassembling the Test Setup ___ 22

Figure 13: The Polyurethane Wing Mounted for Testing in the Same Way as the W1 and W2___ 23

Figure 14: AeroMarine AM 125 Silicon Mold Used with W2 [21] ___ 25

Figure 15: Wing Particulate Trapped in a Mold Created from an Untreated Feather ___ 26

Figure 16: Test Casts of Feather Groups Using Different Release Agents____ 29

Figure 17: The first Stages of Preparing W2 for Molding in the Mold Box ___ 30

Figure 18: L Shaped Corners and Clamps used to maintain the Mold Box's Dimensions ___ 31

Figure 19: Modeling Clay and Play Doh used to Seal the Parting Plain of the Mold __ 32

Figure 20: The First Part of the Two Part Mold during Removal from the Mold Box __ 33

Figure 21: Pouring the Second Part of the Mold onto the Prepared Wing ___ 34

Figure 22: The Completed Two Part Wing Mold ___ 35

Figure 23: Preparing the Two Part Mold for Casting____ 36

Figure 24: The Wing Mold Ready for the Casting Resin to be Poured___ 37

Figure 25: The First Look at the Cast Wing Model____ 38

Figure 26: An Early Comparison of the Original and Cast Wings __ 39

Figure 27: The Final Cast Wing Model Prepared for Testing___ 40

Figure 28: The Cast Wing Model with a Thick Coat of Plasti Dip to Reduce the Surface Geometry___ 41 
Figure 29: A Schematic Representation of the Experimental Test Setup (left) and the Actual Setup Used for Testing

(right) 42

Figure 30: The Experimental Set Up as Seen During Operation ___ 43

Figure 31: An External View of the WVU Closed-Loop Wind Tunnel____ 44

Figure 32: The AeroLab Six-Component Internal Balance Used for Testing ___ 45

Figure 33: Nation Instruments Data Acquisition Unit ___ 46

Figure 34: The Articulated Adaptor Used to Mount the Wing Models to the Six-Component Balance ___ 47

Figure 35: An Image of the Sherline Products Rotary Indexer Used in the Experimental Set Up ___ 48

Figure 36: A DAVID SLS-2 3D Scanner Assembled for Use [24] ___ 49

Figure 37: The AmScope SM-7 Microscope Used to Document the Surface Geometry of the Wing Models [25] _ 50

Figure 38: The Trace of the Cast Wing Model Used to Find the Area and Mean Chord Length___ 51

Figure 39: The Preserved Wing Model Aligned and Prepared for Testing ___ 52

Figure 40: A Comparison of Wing Model Pictures used for Alignment ___ 53

Figure 41: Merged 3D Scans of the Cast (blue) and Polyurethane Coated (green) Wing Models ___ 55

Figure 42: An example of the Distance between Barbs Measured on the Preserved Wing Model using Image Manipulation Software __ 56

Figure 43: Examples of Surface Geometry Measurements ___ 57

Figure 44: An Example of Feather Amplitude and Frequency Measurements Taken from the Preserved Wing Model

- 58

Figure 45: Changes in Covert Feather Amplitude ___ 60

Figure 46: Changes in Secondary Covert Feather Motion ___ 61

Figure 47: Changes in Primary Feather Amplitude ___ 61

Figure 48: Changes in Secondary Feather Amplitude ___ 62

Figure 49: Effects of surface applications on Covert Feather Frequency ___ 63

Figure 50: Effects of Surface Applications on Secondary Covert Feather Frequency ___ 63

Figure 51: Effects of Surface Applications on Primary Feather Frequency ___ 64

Figure 52: Effects of Surface Applications on Secondary Feather Frequency ___ 65

Figure 53: Variations Barb Spacing between Wing Models ___ 68

Figure 54: Variations in Barb Angle Between Wing Models ___ 69

Figure 55: Changes in the Length of Barbules Between Wing Models___ 70

Figure 56: Differences in Barbule Angles Between Wing Models ___ 70

Figure 57: The Changes in Barb and Barbule angles between the Natural (left) and Hair Sprayed (right) Wing Models __ 71

Figure 58: Variations in the Depth of Surface Features Between Wing Models 
Figure 59: A Comparison of the Surfaces of the Cast, Plasti Dipped, Natural, Hair Sprayed and Polyurethane Coated Wing Models 74

Figure 60: Average Resonant Frequencies the Test Feathers with Each Surface Treatment 75

Figure 61: The Deflection of Feathers of Each Treatment Caused by a Known Load 76

Figure 62: Feather Mass after Each Surface Treatment 77

Figure 63: The Average Modulus of Elasticity of Tested Feathers 78

Figure 64: $C_{L}$ Sensitivity to Reynolds Number for the Preserved Wing Model Below 80,000 Re 79

Figure 65: $C_{L}$ Sensitivity to Reynolds Number for the Preserved Wing Model Above 80,000 Re 80

Figure 66: $C_{D}$ Sensitivity to Reynolds Number for the Preserved Wing Model Below 80,000 Re 81

Figure 67: $C_{D}$ Sensitivity to Reynolds Number for the Preserved Wing Model Above 80,000 Re 82

Figure 68: $C_{L}$ Sensitivity to Reynolds Number for the Hair Sprayed Wing Model Below 80,000 Re 83

Figure 69: $C_{L}$ Sensitivity to Reynolds Number for the Hair Sprayed Wing Model Above 80,000 Re 84

Figure 70: $C_{D}$ Sensitivity to Reynolds Number for the Hair Sprayed Wing Model Below 80,000 Re 85

Figure 71: $C_{D}$ Sensitivity to Reynolds Number for the Hair Sprayed Wing Model Above 80,000 Re 86

Figure 72: $C_{L}$ Sensitivity to Reynolds Number for the Polyurethane Coated Wing Model Below 80,000 Re 87

Figure 73: $C_{L}$ Sensitivity to Reynolds Number for the Polyurethane Coated Wing Model Above 80,000 Re 88

Figure 74: $C_{D}$ Sensitivity to Reynolds Number for the Polyurethane Coated Wing Model below 80,000 Re 89

Figure 75: $C_{D}$ Sensitivity to Reynolds Number for the Polyurethane Coated Wing Model Above 80,000 Re 90

Figure 76: Cast Wing Model $C_{L}$ Sensitivity to Reynolds Number Below 80,000 Re 91

Figure 77: Cast Wing Model $C_{L}$ Sensitivity to Reynolds Number Above 80,000 Re 92

Figure 78: Cast Wing Model $C_{D}$ Sensitivity to Reynolds Number Below 80,000 Re 93

Figure 79: Cast Wing Model $C_{D}$ Sensitivity to Reynolds Number Above 80,000 Re 94

Figure 80: Effects of the Reynolds Number on $C_{L}$ of the Plasti Dipped Wing Model Below 80,000 Re 95

Figure 81: Effects of the Reynolds Number on $C_{L}$ of the Plasti Dipped Wing Model Above 80,000 Re 96

Figure 82: Effects of the Reynolds Number on $C_{D}$ of the Plasti Dipped Wing Model Below 80,000 Re 97

Figure 83: Effects of the Reynolds Number on $C_{D}$ of the Plasti Dipped Wing Model Above 80,000 Re 98

Figure 84: Comparison of the Differences in $C_{L}$ between the Preserved and Hair Sprayed Wing Models at 64,000 $\boldsymbol{R e}$ 100

Figure 85: Comparison of the Differences in $C_{D}$ between the Preserved and Hair Sprayed Wing Models at 64,000 Re 100

Figure 86: Comparison of the Differences in $C_{L}$ between the Preserved and Hair Sprayed Wing Models at 78,000 Re

Figure 87: Comparison of the Differences in $C_{D}$ between the Preserved and Hair Sprayed Wing Models at 78,000 $\boldsymbol{R e}$ 101 
Figure 88: Comparison of the Differences in $C_{L}$ between the Preserved and Hair Sprayed Wing Models at 93,000 Re 102

Figure 89: Comparison of the Differences in $C_{D}$ between the Preserved and Hair Sprayed Wing Models at 93,000 Re

Figure 90: Comparison of the Differences in $C_{L}$ between the Preserved and Hair Sprayed Wing Models at 100,000 Re 103

Figure 91: Comparison of the Differences in $C_{D}$ between the Preserved and Hair Sprayed Wing Models at 100,000 Re 103

Figure 92: Comparison of the Differences in $C_{L}$ between the Preserved and Hair Sprayed Wing Models at 110,000 $R e$ 104

Figure 93: Comparison of the Differences in $C_{D}$ between the Preserved and Hair Sprayed Wing Models at 110,000 Re 104

Figure 94: Comparison of the Differences in $C_{L}$ between the Preserved and Polyurethane Coated Wing Models at $64,000 R e$ 106

Figure 95: Comparison of the Differences in $C_{D}$ between the Preserved and Polyurethane Coated Wing Models at $64,000 \mathrm{Re}$ 106

Figure 96: Comparison of the Differences in $C_{L}$ between the Preserved and Polyurethane Coated Wing Models at

78,000 Re 107

Figure 97: Comparison of the Differences in $C_{D}$ between the Preserved and Polyurethane Coated Wing Models at 78,000 Re 107

Figure 98: Comparison of the Differences in $C_{L}$ between the Preserved and Polyurethane Coated Wing Models at 93,000 Re 108

Figure 99: Comparison of the Differences in $C_{D}$ between the Preserved and Polyurethane Coated Wing Models at 93,000 Re 108

Figure 100: Comparison of the Differences in $C_{L}$ between the Preserved and Polyurethane Coated Models at 100,000 Re 109

Figure 101: Comparison of the Differences in $C_{D}$ between the Preserved and Polyurethane Coated Models at $100,000 \mathrm{Re}$ 109

Figure 102: Comparison of the Differences in $C_{L}$ between the Preserved and Polyurethane Coated Models at 110,000 Re

Figure 103: Comparison of the Differences in $C_{D}$ between the Preserved and Polyurethane Coated Models at $110,000 \mathrm{Re}$

Figure 104: Comparison of the Differences in $C_{L}$ between the Hair Sprayed and Cast Wing Models at 64,000 Re 112 Figure 105: Comparison of the Differences in $C_{D}$ between the Hair Sprayed and Cast Wing Models at 64,000 Re 
Figure 106: Comparison of the Differences in $C_{L}$ between the Hair Sprayed and Cast Wing Models at 78,000 Re

Figure 107: Comparison of the Differences in $C_{D}$ between the Hair Sprayed and Cast Wing Models at 78,000 Re_114 Figure 108: Comparison of the Differences in $C_{L}$ between the Hair Sprayed and Cast Wing Models at 93,000 Re

Figure 109: Comparison of the Differences in $C_{D}$ between the Hair Sprayed and Cast Wing Models at 93,000 Re_115 Figure 110: Comparison of the Differences in $C_{L}$ between the Hair Sprayed and Cast Wing Models at 100,000 Re

Figure 111: Comparison of the Differences in $C_{D}$ between the Hair Sprayed and Cast Wing Models at 100,000 Re 116 Figure 112: Comparison of the Differences in $C_{L}$ between the Hair Sprayed and Cast Wing Models at 110,000 Re 117

Figure 113: Comparison of the Differences in $C_{D}$ between the Hair Sprayed and Cast Wing Models at 110,000 Re 118

Figure 114: Comparison of the Differences in $C_{L}$ between the Hair Sprayed and Polyurethane Coated Wing Models at 64,000 Re 119

Figure 115: Comparison of the Differences in $C_{D}$ between the Hair Sprayed and Polyurethane Coated Wing Models at 64,000 Re

Figure 116: Comparison of the Differences in $C_{L}$ between the Hair Sprayed and Polyurethane Coated Wing Models at 78,000 Re

Figure 117: Comparison of the Differences in $C_{D}$ between the Hair Sprayed and Polyurethane Coated Wing Models at $78,000 \mathrm{Re}$

Figure 118: Comparison of the Differences in $C_{L}$ between the Hair Sprayed and Polyurethane Coated Wing Models at 93,000 Re

Figure 119: Comparison of the Differences in $C_{D}$ between the Hair Sprayed and Polyurethane Coated Wing Models at $93,000 \mathrm{Re}$ 122

Figure 120: Comparison of the Differences in $C_{L}$ between the Hair Sprayed and Polyurethane Coated Wing Models at $100,000 \mathrm{Re}$ 122

Figure 121: Comparison of the Differences in $C_{D}$ between the Hair Sprayed and Polyurethane Coated Wing Models at $100,000 \mathrm{Re}$ 123

Figure 122: Comparison of the Differences in $C_{L}$ between the Hair Sprayed and Polyurethane Coated Wing Models at $110,000 \mathrm{Re}$

Figure 123: Comparison of the Differences in $C_{D}$ between the Hair Sprayed and Polyurethane Coated Wing Models at $110,000 \mathrm{Re}$

Figure 124: Comparison of the Differences in $C_{L}$ between the Cast and Plasti Dipped Wing Models at 64,000 Re_125 Figure 125: Comparison of the Differences in $C_{D}$ between the Cast and Plasti Dipped Wing Models at 64,000 Re_ 125 
Figure 126: Comparison of the Differences in $C_{L}$ between the Cast and Plasti Dipped Wing Models at 78,000 Re

Figure 127: Comparison of the Differences in $C_{D}$ between the Cast and Plasti Dipped Wing Models at 78,000 Re

Figure 128: Comparison of the Differences in $C_{L}$ between the Cast and Plasti Dipped Wing Models at 93,000 Re_127 Figure 129: Comparison of the Differences in $C_{D}$ between the Cast and Plasti Dipped Wing Models at 93,000 Re_128 Figure 130: Comparison of the Differences in $C_{L}$ between the Cast and Plasti Dipped Wing Models at 100,000 Re

Figure 131: Comparison of the Differences in $C_{D}$ between the Cast and Plasti Dipped Wing Models at 100,000 Re

Figure 132: Comparison of the Differences in $C_{L}$ between the Cast and Plasti Dipped Wing Models at 110,000 Re 129

Figure 133: Comparison of the Differences in $C_{D}$ between the Cast and Plasti Dipped Wing Models at 110,000 Re 130

Figure 134: Comparison of the Differences in $C_{L}$ between the Polyurethane Coated and Cast Wing Models at 64,000 $R e$

Figure 135: Comparison of the Differences in $C_{D}$ between the Polyurethane Coated and Cast Wing Models at 64,000 $R e$

Figure 136: Comparison of the Differences in $C_{L}$ between the Polyurethane Coated and Cast Wing Models at $78,000 \mathrm{Re}$

Figure 137: Comparison of the Differences in $C_{L}$ between the Polyurethane Coated and Cast Wing Models at $78,000 \mathrm{Re}$

Figure 138: Comparison of the Differences in $C_{L}$ between the Polyurethane Coated and Cast Wing Models at 93,000 Re

Figure 139: Comparison of the Differences in $C_{D}$ between the Polyurethane Coated and Cast Wing Models at 93,000 Re 134

Figure 140: Comparison of the Differences in $C_{L}$ between the Polyurethane Coated and Cast Wing Models at $100,000 \mathrm{Re}$ 134

Figure 141: Comparison of the Differences in $C_{D}$ between the Polyurethane Coated and Cast Wing Models at $100,000 \mathrm{Re}$ 135

Figure 142: Comparison of the Differences in $C_{L}$ between the Polyurethane Coated and Cast Wing Models at $110,000 \mathrm{Re}$

Figure 143: Comparison of the Differences in $C_{D}$ between the Polyurethane Coated and Cast Wing Models at $110,000 \mathrm{Re}$

Figure 144: Comparison of the Differences in $C_{L}$ between the Polyurethane Coated and Plasti Dipped Wing Models at 64,000 Re 
Figure 145: Comparison of the Differences in $C_{D}$ between the Polyurethane Coated and Plasti Dipped Wing Models at 64,000 Re

Figure 146: Comparison of the Differences in $C_{L}$ between the Polyurethane Coated and Plasti Dipped Wing Models at $93,000 \mathrm{Re}$ 138

Figure 147: Comparison of the Differences in $C_{D}$ between the Polyurethane Coated and Plasti Dipped Wing Models at $93,000 \mathrm{Re}$ 138

Figure 148: Comparison of the Differences in $C_{L}$ between the Polyurethane Coated and Plasti Dipped Wing Models at $100,000 \mathrm{Re}$ 139

Figure 149: Comparison of the Differences in $C_{D}$ between the Polyurethane Coated and Plasti Dipped Wing Models at $100,000 \mathrm{Re}$ 139

Figure 150: Comparison of the Differences in $C_{L}$ between the Polyurethane Coated and Plasti Dipped Wing Models at 110,000 Re 140

Figure 151: Comparison of the Differences in $C_{D}$ between the Polyurethane Coated and Plasti Dipped Wing Models at $110,000 \mathrm{Re}$ 140

Figure 152: A Comparison of the $C_{L}$ Curve for each Model at 64,000 Re 142

Figure 153: A Comparison of the $C_{D}$ Curve for each Model at 64,000 Re 143

Figure 154: A Comparison of the L/D Curve for each Model at 64,000 Re 143

Figure 155: A Comparison of the $C_{L}$ Curve for each Model at 110,000 Re 144

Figure 156: A Comparison of the $C_{D}$ Curve for each Model at 110,000 Re 145

Figure 157: A Comparison of the L/D Curve for each Model at 110,000 Re 145 


\section{List of Tables}

Table 1: Feather Mold Release Agents and Their Test Results ...................................................................27

Table 2: Photo Documentation of Release Agent Test Results ....................................................................28

Table 3: Change in Feather Motion with the Application of Hair Spray ..........................................................65

Table 4: Feather Motion Reductions Caused by the Application of Polyurethane Spray.........................................67

Table 5: Measured Changes in Surface Geometry between Wing Models ...................................................... 73

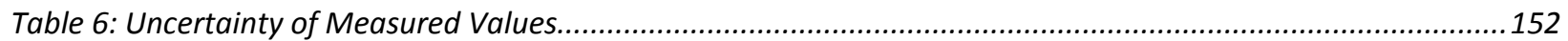

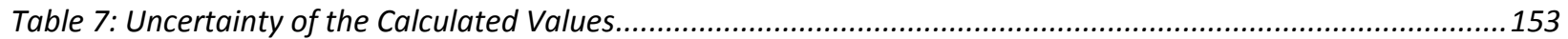

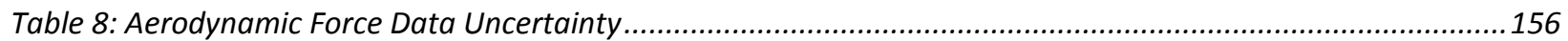

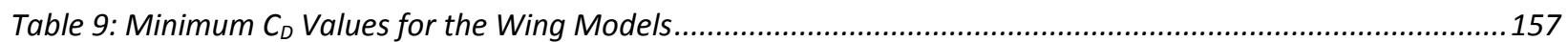

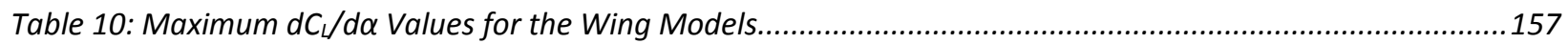

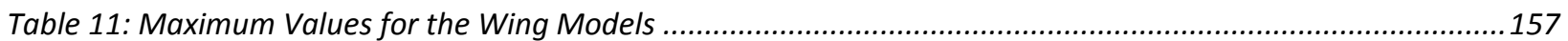




\section{Nomenclature}

\section{Abbreviations}

$\begin{array}{ll}\text { Amp } & \text { Amplitude } \\ \text { AR } & \text { Aspect Ratio } \\ \text { CNC } & \text { Computer Numerical Control } \\ \text { DAQ } & \text { Data Acquisition Unit } \\ \text { FSI } & \text { Fluid Structure Interaction } \\ \text { STL } & \text { Stereo Lithography } \\ \text { W1 } & \text { Preserved wing model } \\ \text { W2 } & \text { Hair Sprayed Wing Model } \\ \text { W3 } & \text { Polyurethane Coated Wing Model } \\ \text { W4 } & \text { Cast Wing Model } \\ \text { W5 } & \text { Plasti Dip Coated Cast Wing Model } \\ \text { WVU } & \text { West Virginia University } \\ \text { 3D } & \text { Three Dimensional }\end{array}$

\section{Symbols}
A
Area
B
Wing Span
$\mathrm{C}_{\mathrm{L}}$
Coefficient of Lift
$\mathrm{C}_{\mathrm{D}}$
Coefficient of Drag
$\mathrm{H}$
Height
L
Length
$\operatorname{Re}_{\mathrm{x}}$
Reynolds number with respect to generic length quantity $\mathrm{x}$ 


\begin{tabular}{ll}
$\mathrm{T}$ & Temperature \\
$\mathrm{P}$ & Pressure \\
$\mathrm{V}$ & Individual Gas Constant \\
$\mathrm{W}$ & Velocity \\
$\alpha$ & Width \\
$\delta$ & Angle of Attack \\
$\mu \mathrm{m}$ & Depth of Surface Features \\
$\zeta$ & Micron \\
$\beta$ & Barb to Barb Distance \\
$\Gamma$ & Barb Angle \\
$\lambda$ & Barbule Length \\
$\exists$ & Barbule to Barbule Distance \\
$\zeta$ & Barbule Angle \\
\hline
\end{tabular}

\section{Subscripts}

$\operatorname{Max}$

Maximum Value

Min

Minimum Value

\section{Notations}

$\Delta$

Difference 


\section{Chapter 1: Problem Statement and Research Objectives}

\section{Problem Statement}

At some point, every child has laid on his or her backs in a field on a sunny day and gazed at the sky and the things that fill it. Most children pay attention to the clouds and what objects their shapes resemble. However, a few children take notice of the birds in the sky and wonder how they fly. This scenario has happened in one way or another throughout the development of mankind. However, one question about how those birds we watch fly continues to go unanswered: What effects do the feathers of a bird have?

The world of unmanned aerial vehicles (UAVs) and micro aerial vehicles (MAVs) is continuing to develop. This has resulted in the demand for new and revolutionary methods to increase the performance of these vehicles operating in the same low Reynolds number (Re) range $[1,2]$. As a result, researchers are once again looking to characteristics of avian flight for design inspirations, with the end goal of improving the performance of UAVs operating at the same Reynolds numbers.

Birds are often seen flying through the air, performing spectacular maneuvers with ease. Once characteristic of birds that may contribute to the high levels of control and agility casually displayed by birds is feathers. Although some attention has been given to the overall effects of feathers, the effects of free feather motion and the millions of tiny grooves and surface texture resulting from the feather structure have yet to gain the spotlight.

In order to shed light on the effects of freely moving feathers, the research and results described herein work to explain the effects of freely moving feathers on a wing. As a result, new 
theories to answer the question, "How important are freely-moving feathers for biomimetic flight?” can be found.

\section{Objectives}

The primary objective of this study was to understand the performance effects of feathers on a bird's wing. However, in order to complete this task the work was divided into two the following two separate, interdependent underlying objectives.

1. Determine if fluid-structure interaction (FSI) in the form of the passive movement of feathers attached to a bird wing has a measurable effect on the performance of that wing.

2. Determine the influence of microscopic surface geometry on the aerodynamic performance of a bird wing. 


\section{Chapter 2: Review of Relevant Literature}

\subsection{Introduction}

Animals, as well as other fauna and even flora, have long been the inspiration for the inventions of humans. By observing the natural behaviors and physical characteristics of nature’s creatures, some of the world's most impacting and inspiring inventions have been made.

“The genius of man may make various inventions, encompassing with various instruments one and the same end; but it will never discover a more beautiful, a more economical, or a more direct one than nature's, since in her inventions nothing is wanting and nothing is superfluous."-Leonardo Da Vinci [3]

Nature can be thought of as an excellent inventor and was referred to as "the great master teacher” by Heinrich Hertel when explaining the relation between technology and biology [2]. Through many generations of trial and error, plants and animals have been adapted or naturally designed to be highly efficient biological machines [4, 5]. This process has resulted in generations of trial and error to serve as design iterations to develop unique adaptations to give animals various advantages. One such result of natural adaptations is the flight of birds.

\subsection{Inspirations of Avian Flight in History}

The ability of birds to soar through the skies, while man was forced to observe from the ground has baffled mankind for centuries. Great thinkers and inventors, like Leonardo da Vinci and Otto Lilienthal, attempted to learn the secrets of avian flight through observation. These observations were made in hopes of applying what they could learn to their own inventions. 
Da Vinci spent a considerable amount of time observing and analyzing the flight of birds and bats. He was even known to purchase and release birds so that he could watch as they took off and flew away. Eventually, Da Vinci attempted to apply his observations of bird flight to design the world's first known flying machine concept shown in Figure 1.

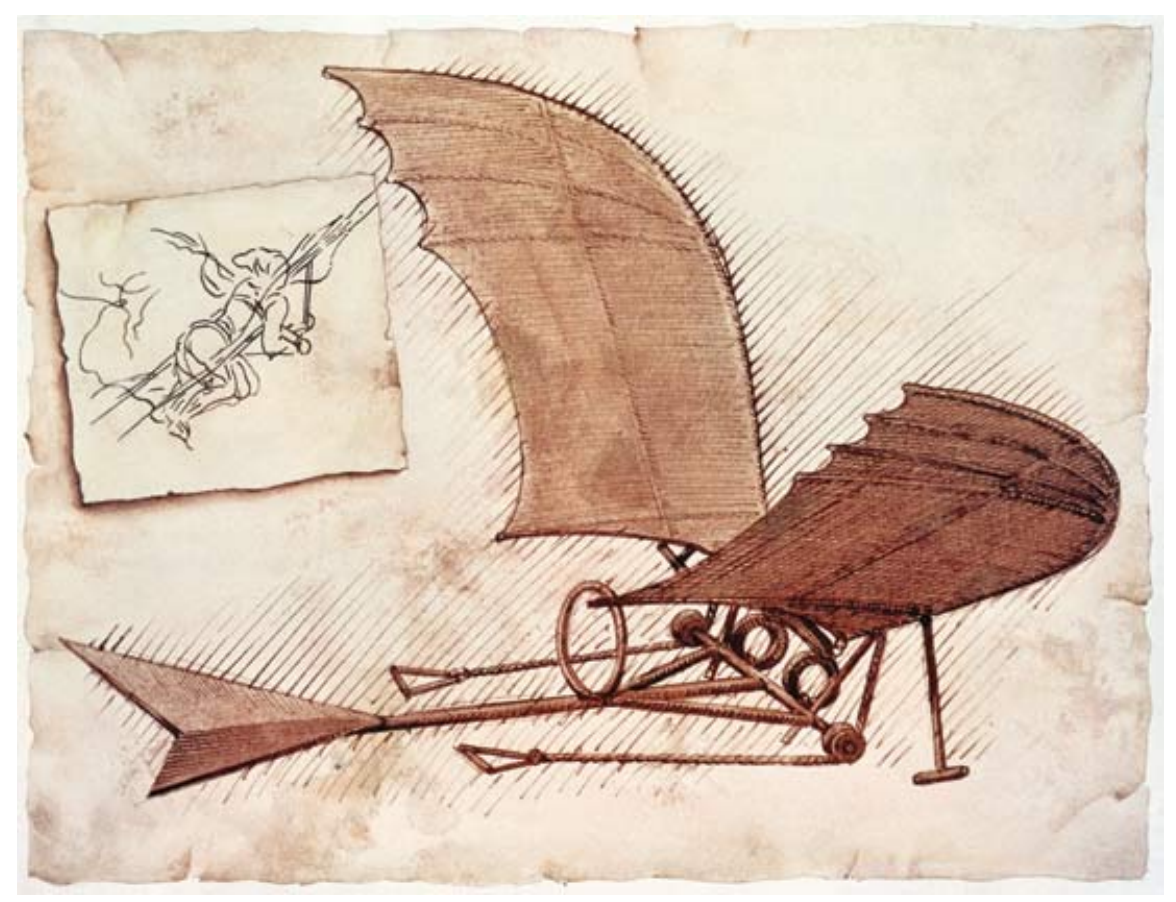

Figure 1: A Sketch of the Da Vinci Flying Machine [6]

The Da Vinci flying machine resembled birds and bats in many aspects of its design. The machine was designed as an ornithopter. By using this ornithopter design, the shape of the wings and their support structure were modeled after those of a bat. In this system the wings were "flapped" to propel the vehicle in flight. Leonardo da Vinci based his design on bats due to their lack of feathers since he knew he could not reproduce the feathers of a bird. Therefore, he based most of his design on a similar flying creature that did not have feathers [3].

Several hundred years later, Otto Lilienthal was similarly inspired by the flight of biological organisms. Like Da Vinci, Lilienthal (also known as "the bird man") applied 
observations of bird flight to the design of eighteen different flying machines resembling modern hang gliders [7]. Lilienthal was the first to understand the importance of the shape of the wing section. Through his work he realized that the aerodynamics of a wing were improved when the correct amount of camber and thickness were applied to a wing [2]. He applied this knowledge to build a series of gliders. Using one of these gliders, Lilienthal was able to climb to an altitude of 80 meters. As a result of his research's success, Otto Lilienthal's book "Der Vogelflug als Grundlage Der Fliegekunst" or "The Flight of Birds as the Basis of Aviation" has become a fundamental component of modern aviation design and education [2].

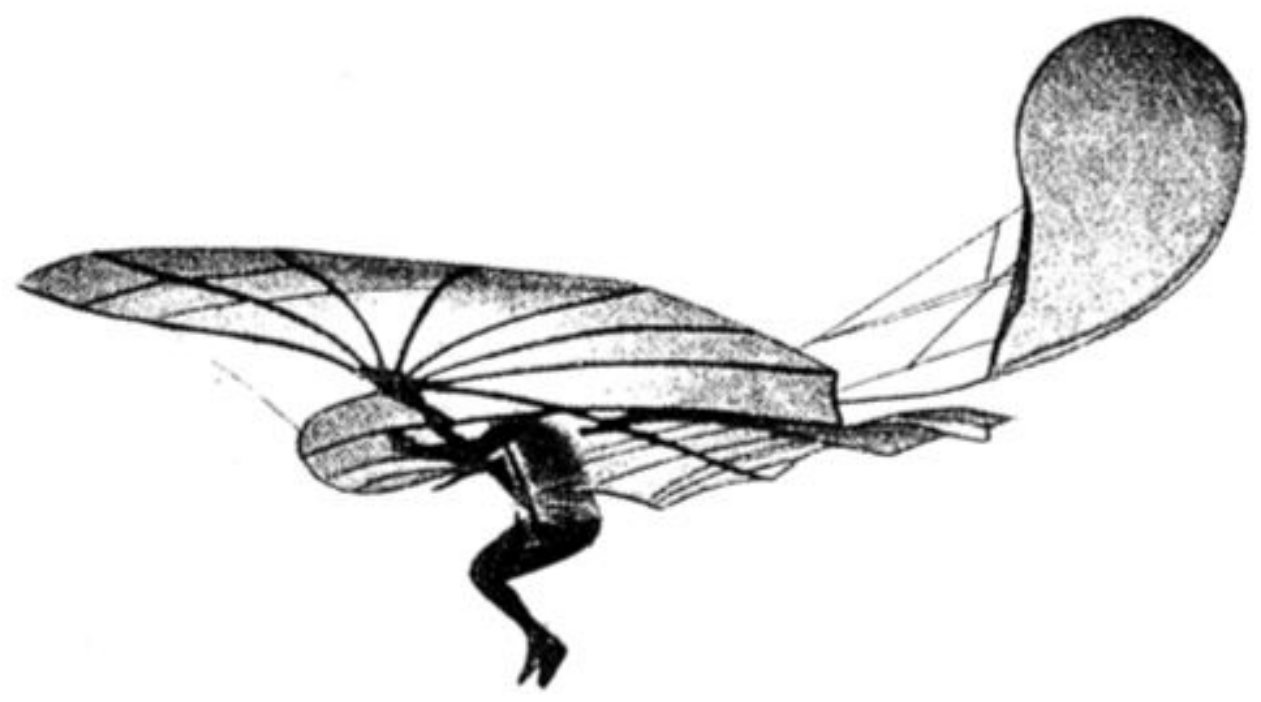

Figure 2: One of Lilienthal's Final Glider Designs Inspired by Birds [2]

Lilienthal's observations of birds served as his inspiration. Later, the results of those observations would serve as his demise. After building several successful gliders, Lilienthal applied a motor to a design [2]. However, he failed to develop a method to steer and control his aircraft. As a result, his glider stalled during flight causing him to crash. Lilienthal later died of 
the wounds he received during the crash [8]. His research is commonly believed to have directly inspired Orville and Wilber Wright who are documented to have made the first successful manned and motorized flight [7].The design the Wright brothers used for their bi-plane incorporated a cambered wing profile modeled after Lilienthal's glider design [2, 7].

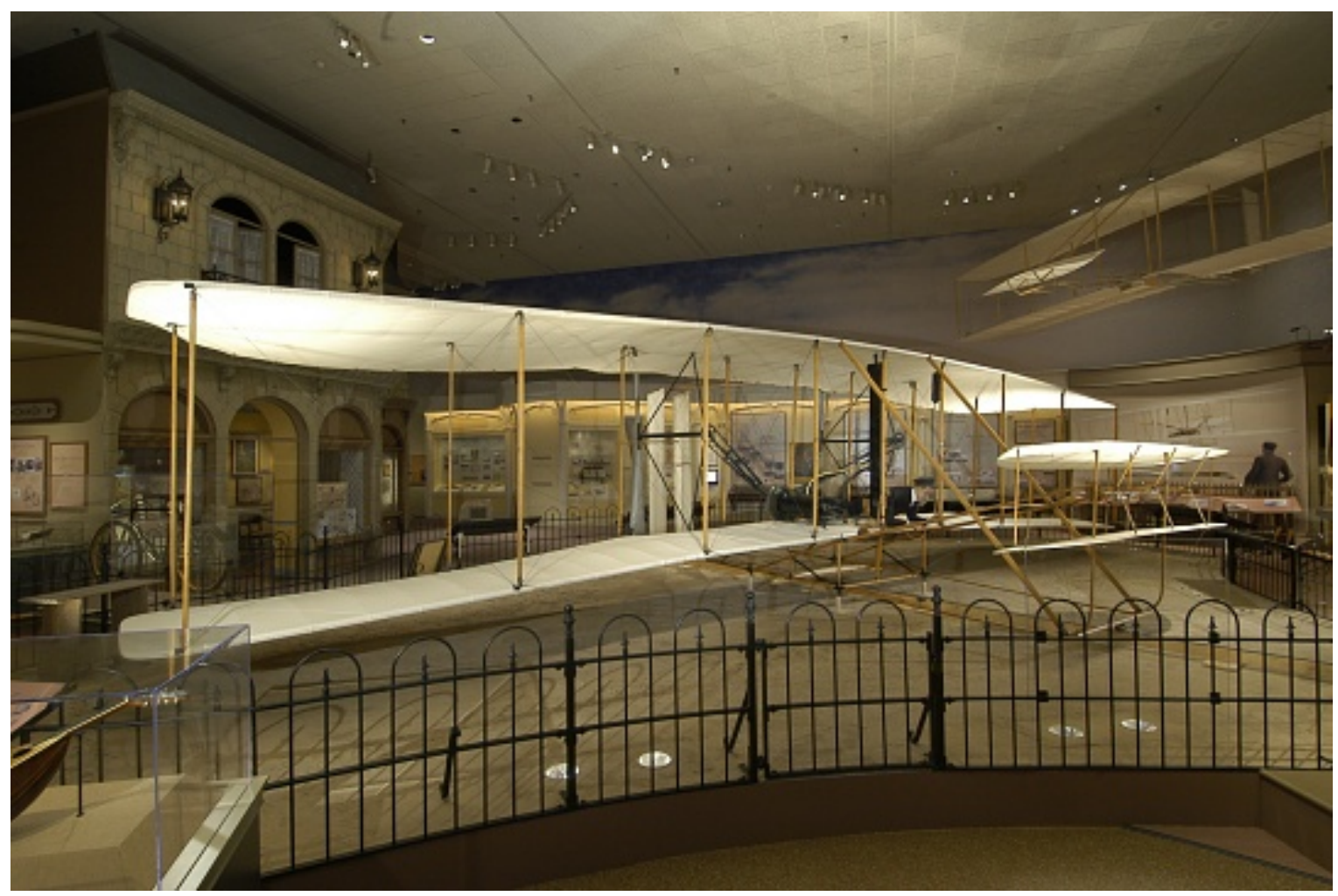

Figure 3: A Model of the 1903 Wright Flyer at the Smithsonian National Air and Space Museum [9]

The key to the Wright brothers' success was their ability to control the aircraft in flight. They accomplished this using a wing warp system. The Wright brothers were able to develop this system though observations of buzzards flying in their home state of Ohio [8].

As with Leonardo da Vinci, Otto Lilienthal, the Wright brothers, and other pioneers of technology, the flight of birds and other flying species have directly influenced and inspired research that has helped to shape our modern world. However, as modern aviation has 
progressed, the main focus of aircraft has been set on flight conditions that birds and other natural fliers cannot attain such as high altitudes, and speeds.

Small unmanned aerial vehicles (UAV) have recently been the subject of numerous news headlines. As unmanned technology has developed, resulting designs have become smaller and smaller over time. As a result, the flight conditions many of these small UAV are intended for are the same as natural flyers. The capabilities of small UAVs operating within the same Reynolds numbers are birds drastically decreased when compared to those of birds. Therefore, researchers are now turning their attention back to natural fliers for inspiration. Their hope is that hidden somewhere in the fundamental flight characteristics and features of a bird, lay the necessary keys that will unlock more efficient and more advanced unmanned aerial vehicles and micro aerial vehicles $[1,10]$.

\subsection{Fundamentals of Avian Flight}

The mechanics of bird flight can be broken into the following primary phases: flapping,

gliding, maneuvering, and in some cases hovering $[11,12]$. The flapping phase derives its name from the flapping motion of a bird's wing during this phase. By flapping its wings, a bird can propel itself through the air. However, this flapping process is more complex than it initially sounds.

Each flap of a bird's wings can be broken into two strokes: the downstroke, and the upstroke. Forward thrust can be produced during either stroke [11]. Avian accomplish this by manipulating the shape and pitch of the wing throughout the flapping cycle $[11,12]$. The downstroke is the primary producer of both lift and forward thrust, whereas the upstroke 
produces primarily lift. The upstroke produces little to no forward thrust due to the required shape of the wing during upstroke.

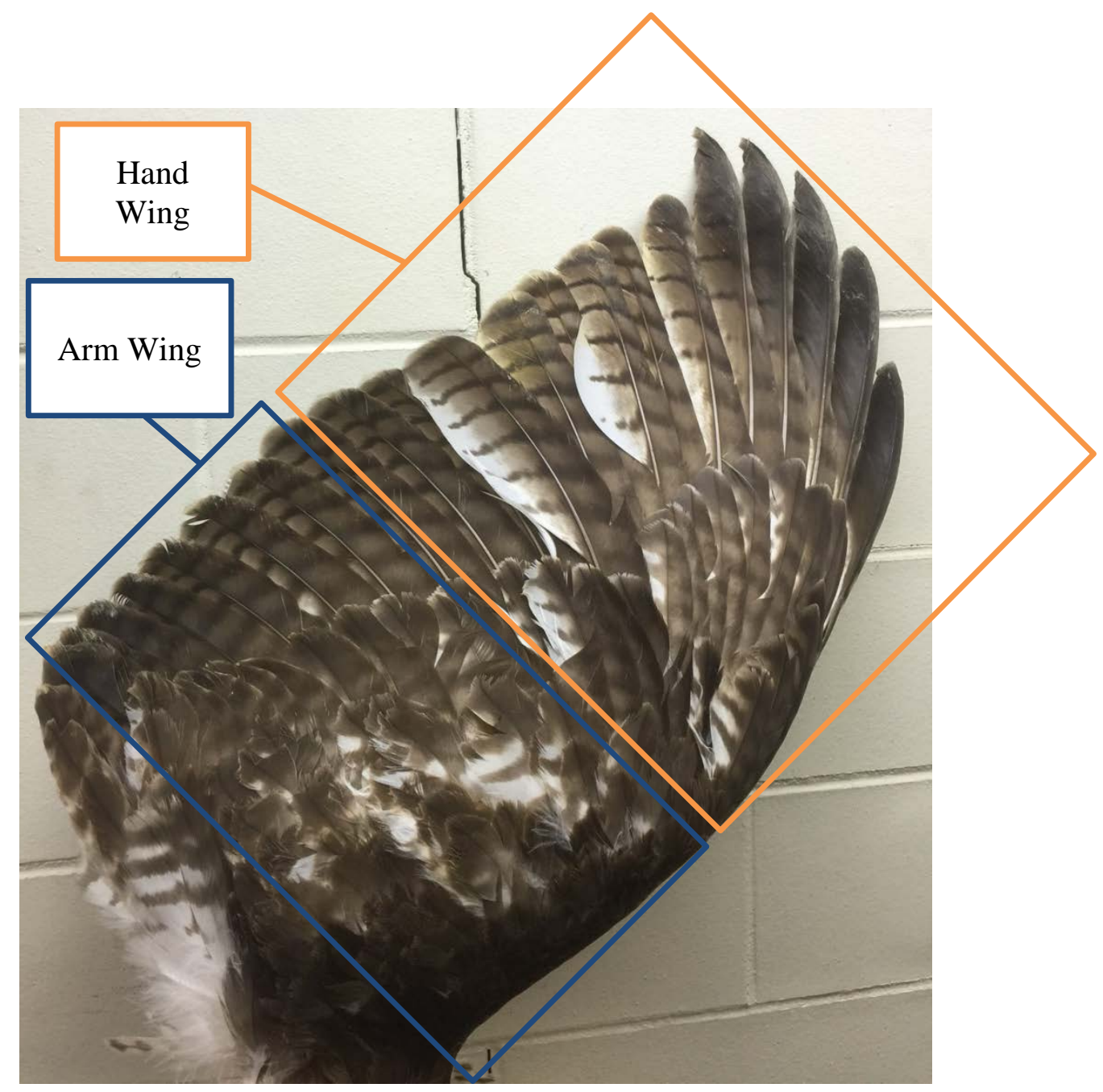

Figure 4: The Major Wing Regions as Seen on a Red Tailed Hawk Wing [13]

The tip of the wing beyond the elbow is called the hand wing and produces the most forward thrust [13]. During the upstroke portion of the flapping cycle, this portion of the wing is withdrawn. Only the portion of the wing from the shoulder to the elbow or the arm wing is known to produce primarily lift, is extended and effective [12, 13]. Gliding or soaring is the 
phase of flight in which the bird is doing little or no flapping. This phase is employed usually to reserve energy or to rest.

\subsection{Overview of Avian Feather Structure}

Avian feathers have different shapes, colors, and specialized structures that can vary from species to species. The major variations between the feathers of different species depend on the natural adaptations the species has developed. However, the basic structure of feathers remains the same.

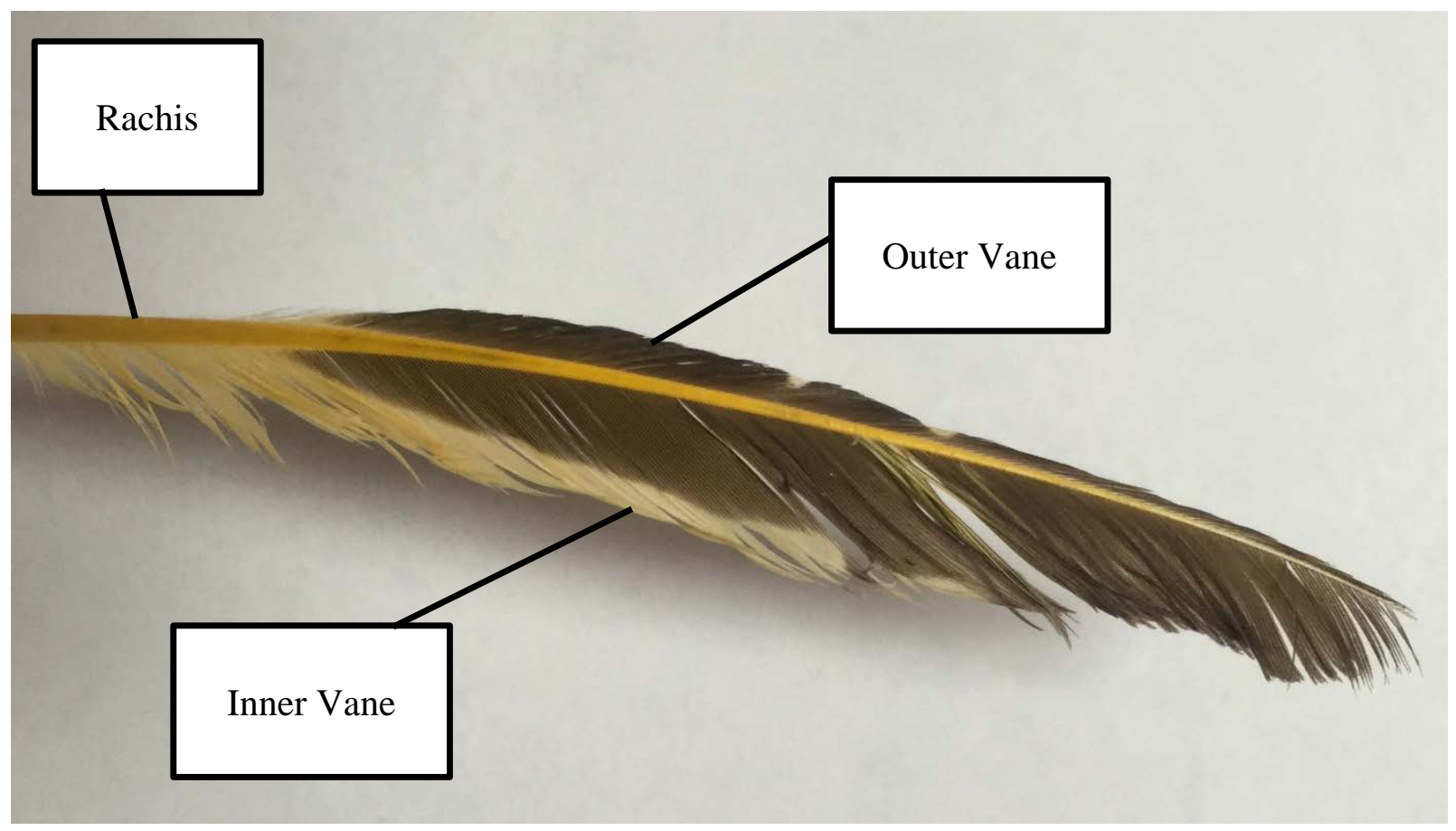

Figure 5: The Major Feather Structures as Seen on the Feather of a Finch

Feathers such as the one seen in Figure 6 are primarily made up of the protein keratin. They are also considered to be dead and not a living organ, like the epidermis, or skin, of an 
animal [12]. Therefore, the feathers of a bird do not repair themselves and must be replaced at various intervals in a process called molting [14].

The primary shaft of the feather is separated into two parts, the calamus and the rachis. The calamus is the hollow portion of the shaft nearest the base of the feather. When attached to the bird, the calamus is the portion of the feather emerging from the follicle in the skin of the bird. Farther from the base of the feather, the hollow cavity of the calamus is filled with a spongey substance. The beginning of this portion of the feather to the tip of the shaft is called the rachis. The rachis portion of the shaft consists of two parts. One part is the hard outer shell of the shaft. It is called the cortex. The inner part of the rachis consists of a spongey material called pith. This fills the inner cavity of the cortex [12].

Extending from the rachis are the inner and outer vanes. The inner and outer vanes are named based upon their orientation. The vane protruding toward the leading edge of the bird's wing is called the outer vane. The inner vane is the vane that protrudes toward the trailing edge. Vanes are rarely straight but are more commonly curved downward or sideways. However, many vanes curve in more than one direction.

Vanes are composed of individual rows of parallel barbs as seen in Figure 7. Barbs are usually angled toward the feather tip. The stiffness of the vane is dependent upon the spacing of the barbs. In stiffer portions of the vane near the feather tip, the spacing between the barbs is the smallest. As the distance between the barbs increases, the flexibility of the vane also increases. 


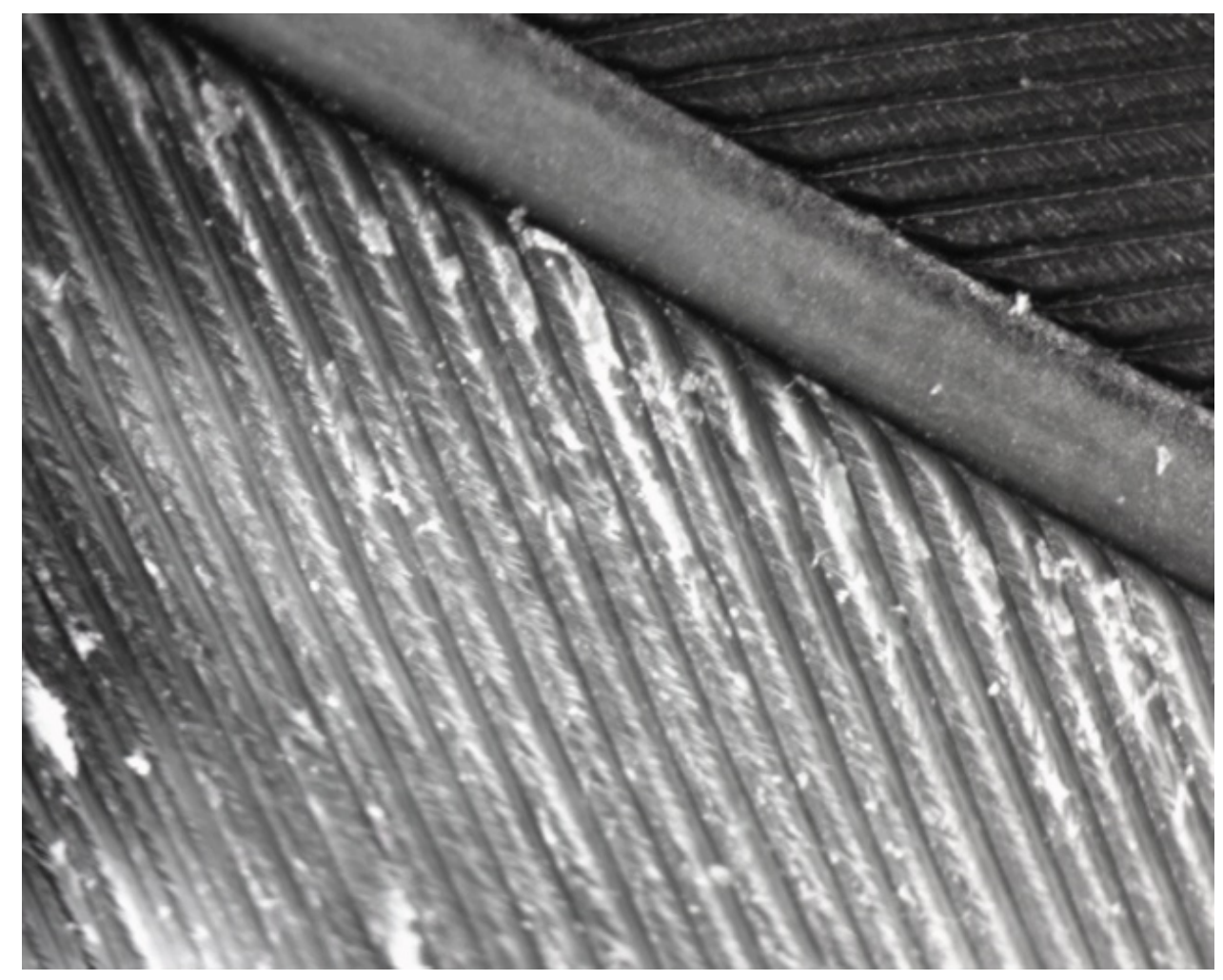

Figure 6: The Barbs of a Red Tailed Hawk Feather's Vane as Seen Under a Microscope

The basic structure of the feathers of a bird, such as the shape, types of barbules, and barb spacing, change. The variations in these parameters are based on the role and location of the feathers on a wing. Most flying birds have four major feather groups that impact flight: the coverts, primaries, secondaries, and alula as seen in Figure 7 [10, 11, 13]. 


\subsection{Feather Types}

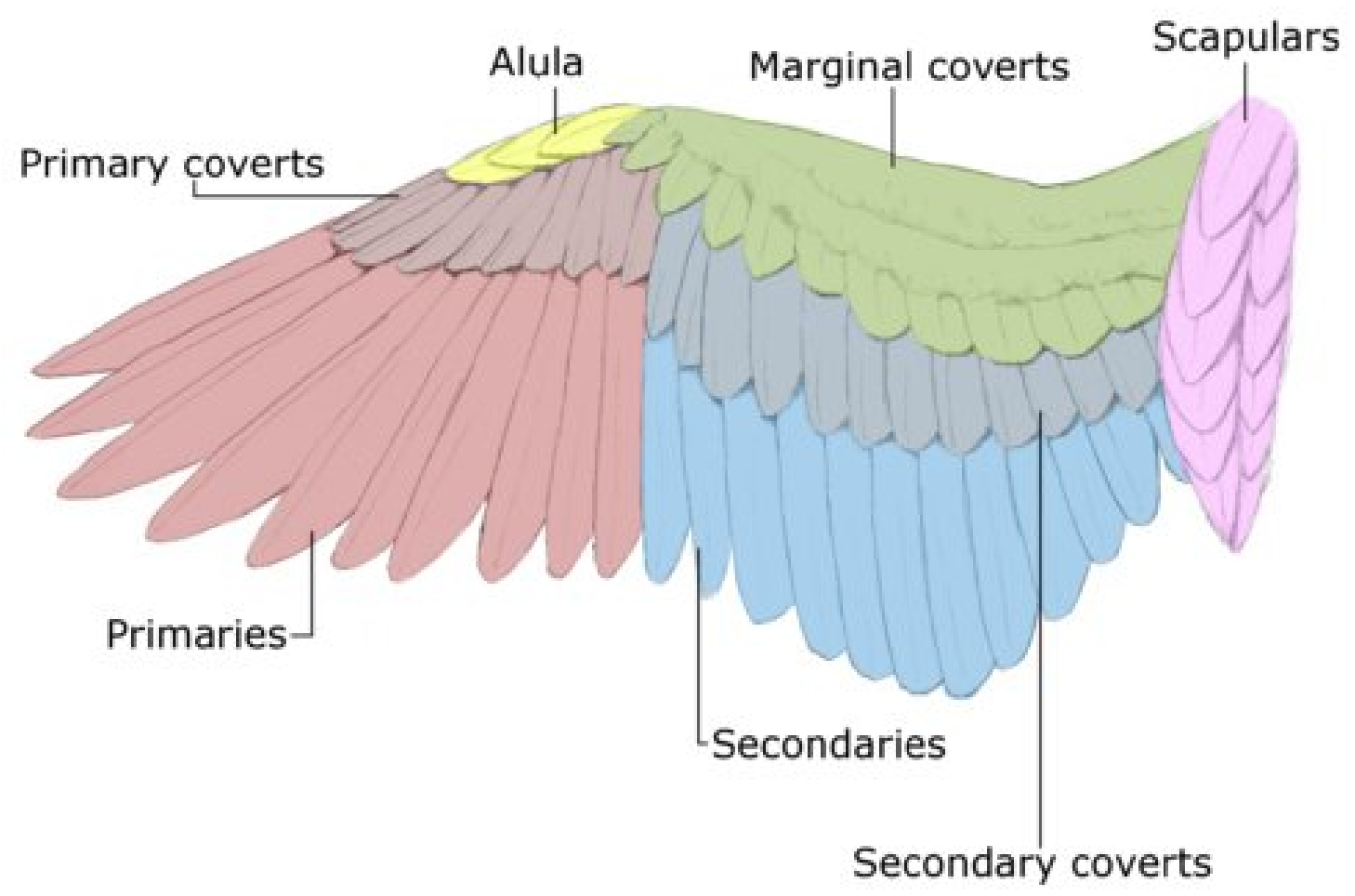

Figure 7: The Major Feather Groups of a Bird [15]

\subsubsection{Primary Feathers}

Primary feathers are located on the hand wing. These feathers are sometimes thought of as the fingers. As shown in the differences between land soaring and sea soaring birds, the shape and spacing of the primaries have an effect on the wing tip vortices. As a result, the shape and spacing of the primary feathers can cause a reduction in drag. This is especially noticeable with the slotted wing tips of land soaring birds $[11,16]$. 


\subsubsection{Secondary Feathers}

The secondary feathers are located along the trailing edge of the wing between the primaries and the scapulars. These feathers help the bird to generate lift and sustain itself during flight [17]. However, experiments have been conducted with secondary feathers removed or restrained on a bird. The flights of these birds showed that flight was still possible, but some control was lost $[12,17]$.

\subsubsection{Alula Feathers}

The alula feathers are located in a small section of feathers located between the coverts and the primaries at the leading edge of the wing. These feathers are the beginning of the hand wing and are often thought of as the thumb. Alula feathers are actively controlled by the bird [10]. Studies have shown that birds use the alula as a high-lift device. A high-lift device can refer to several different approaches to increase flight performance, such as increasing the airfoil camber, controlling the boundary layer, and increasing the airfoil chord. However, alulae serve as a high-lift device by controlling the boundary layer "making it more resistant to adverse pressure gradients” $[10,18]$.

High speed imagery of a Steppe Eagle has shown that the active extension of the alulae also affects the marginal coverts located at the leading edge of the arm wing. After alulae have been extended, cover feathers begin to extend. The coverts extend first nearest the alulae and cascades along the leading edge to the scapular feathers near the body of the bird. Together they work as a leading edge flap. This process repeatedly occurred during the final moments as the trained eagle used in the test performed a landing maneuver. However, only high speed video of this phenomenon was taken. Therefore, the researchers could only hypothesize actual effects of this process [10]. 


\subsubsection{Covert Feathers}

Covert feathers make up the center portion of the wing between the leading edge and the secondaries. These feathers are believed to act independent of a bird's nervous system. In other words, covert feathers are believed to be passively controlled during flight. This is attributed to the randomness of their recorded movement during flight scenarios in which they become active [10].

Through observations of birds in flight, it has been noticed that covert feathers are most active when the bird is gliding with its wings at high angles of attack. This observation has led many experts to believe these feathers act as a high-lift device or stabilizer during these situations. A similar hypothesis suggests these feathers act as a type of passive flap that works to keep air flow over the upper surface of the wing attached at increased angles of attack. As a result, similar systems have been investigated for use on MAVs. However, a measure of this effect on a bird's wing has not yet been found [1, 10].

\subsection{Effects of Feather Motion}

Although it has been shown that the shape, size, and make up of a feather affect the wing's overall performance, a deficiency in our knowledge still exists. We know how each feather group effects flight. However, the question "what effect does the free movement of these feathers have on the wing?” continues to go unanswered. 


\section{Chapter 3: Experimental Setup}

In order to complete the objectives of this study, several wing models had to be created to compare the effects of differences in surface geometry and feather motion. As a result, five variations of the same wing were tested in the WVU closed loop wind tunnel. Each of these wings was prepared or reproduced to exhibit varying levels of feather motion and variations in the surface geometry. The processes explained in this chapter cover how each wing was prepared and tested as well as the equipment used.

\subsection{Harvesting of the Test Wing}

To complete the objectives, a harvested wing was chosen to be the foundation of this research. The wing used was harvested from the cadaver of a young adult red-tailed hawk (Buteo jamaicensis) carcass. The cadaver was donated by a local rehabilitation center for wounded wildlife, the Avian Conservation Center of Appalachia [19]. No animals were harmed or euthanized for the purpose of this research.

The wing was separated from the body of the bird at the proximal end of the humorous bone, near the shoulder of the bird. In order to use the wing in any test set up, the aluminum mounting fixture shown in Figure 9 was then attached to the proximal end of the humorous bone. During this process all covert, primary, secondary, and alula feathers were kept intact. 


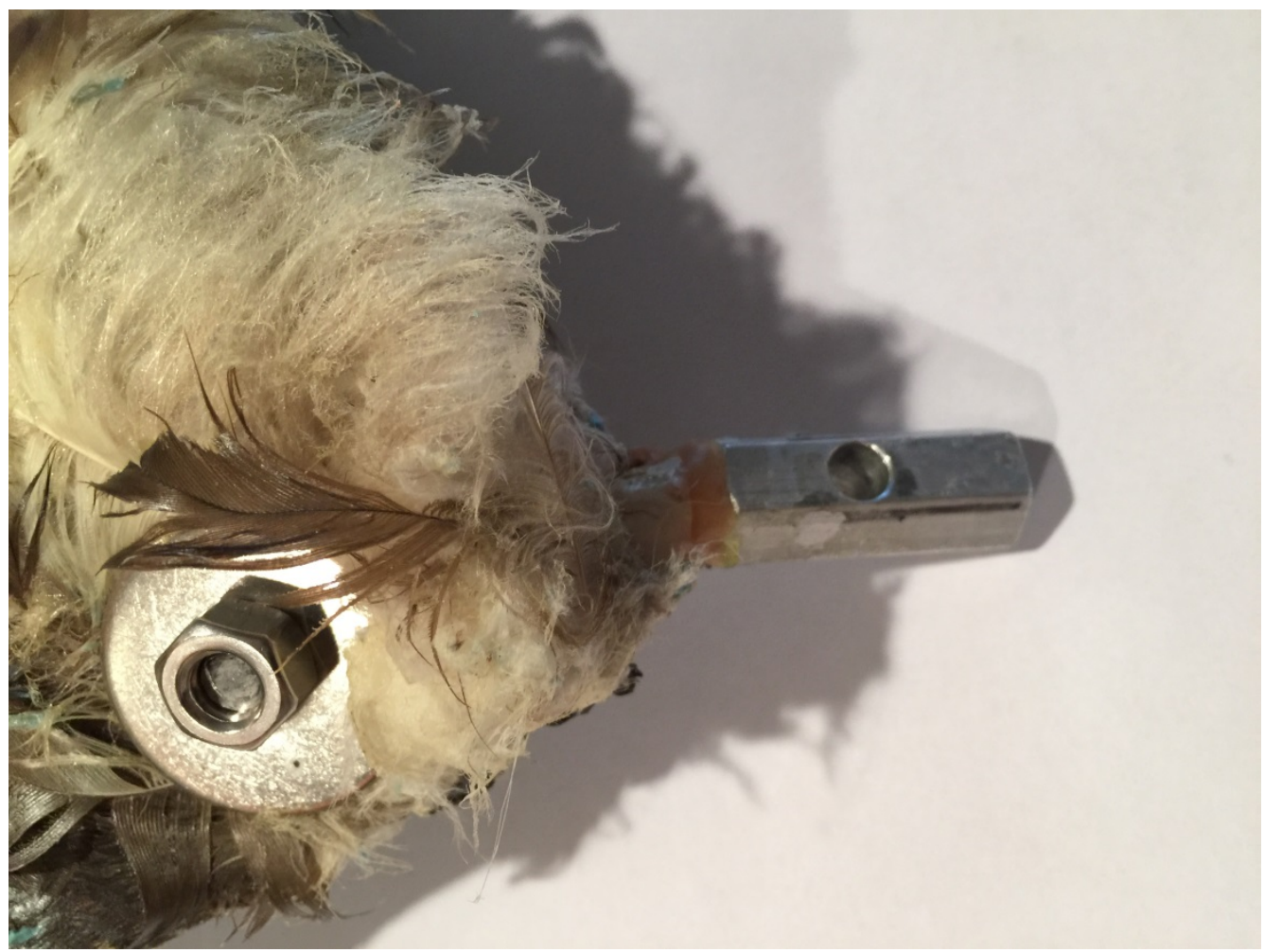

Figure 8: The Aluminum Mounting Fixture Attached to the Base of the Wing for Testing

\subsection{Initial Preparation of the Test Wing}

In order to use the wing for testing, several challenges had to be overcome. As with any wild animal, harmful parasites such as lice and fleas were likely to be living on the wing. The wing would also start to decay if left untreated at room temperature. Therefore, the first step in preparing the wing for testing was to sanitize and preserve the wing in order to make it safe for researchers to handle.

Another problem associated with using this natural wing in a thawed state was the flexibility of the wing. As with any deceased animal, the extremity was limp and could not hold a suitable shape for testing on its own. Yet another problem present was the decay of the wing. The more time the wing spent out of the freezer, the more the wing would decay. It was known 
that if this decay was allowed to continue, potentially harmful bacteria would begin to grow on the wing. The condition of the wing would also deteriorate over time.

\subsection{Preservation and Sanitization of the Wing}

To overcome these issues a natural method was developed to dry and preserve the wings. The method that was developed uses corn meal, dry rice, and disodium tetraborate in the form of borax laundry detergent as desiccants. All three agents actively absorb moisture. The disodium tetraborate also killed any parasites that may be on or in the wing. Together these materials acted to slowly dehydrate the wing without using arsenic or other harmful chemicals used by professional taxidermists. This preservation process involved two main phases. During the first phase, the wing was deep frozen below $-10^{\circ} \mathrm{F}$. The wing was frozen for two reasons. The first reason was for storage. This prevented the wing from decaying. The second reason was to begin killing parasites that may be living on or in the wings [20].

The final phase began when the wing was removed from the freezer. After thawing, the wing was attached to a supportive wire mesh as seen in Figure 9. During this step, the wire mesh acted as a mounting surface. This allowed a wide range of potential mounting points for the wire constraints to provide flexibility in both the location and direction of constraints. As a result, the wing could be conformed to a desired shape. For the purposes of this study, this permitted the wings to be dried in an outstretched position similar to the shapes of wings observed during the gliding phase of flight. 


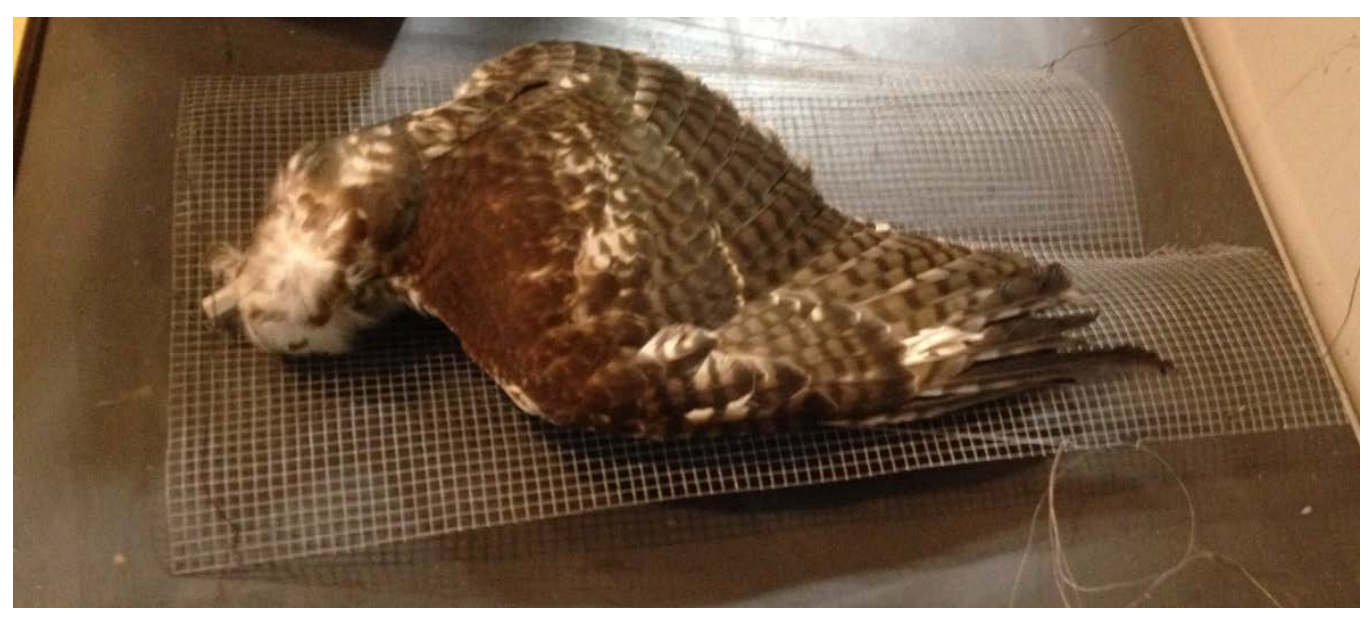

Figure 9: A Red Tailed Hawk Wing Fix to Wire Mesh with Fishing Line Restraints to Control the Wing Shape

For the desiccants to effectively remove a suitable amount of moisture from the wings, the wings were immersed in the materials, as seen in Figure 10. Here, it remained for just over thirty days. The preserved wing resulting from this process could then be left unrefrigerated for extended periods with almost no biologic degeneration. However, as with all taxidermy, some deterioration will occur over the course of time. The wings were also parasite free as a result of freezing the wing and the extended exposure to disodium tetraborate. 


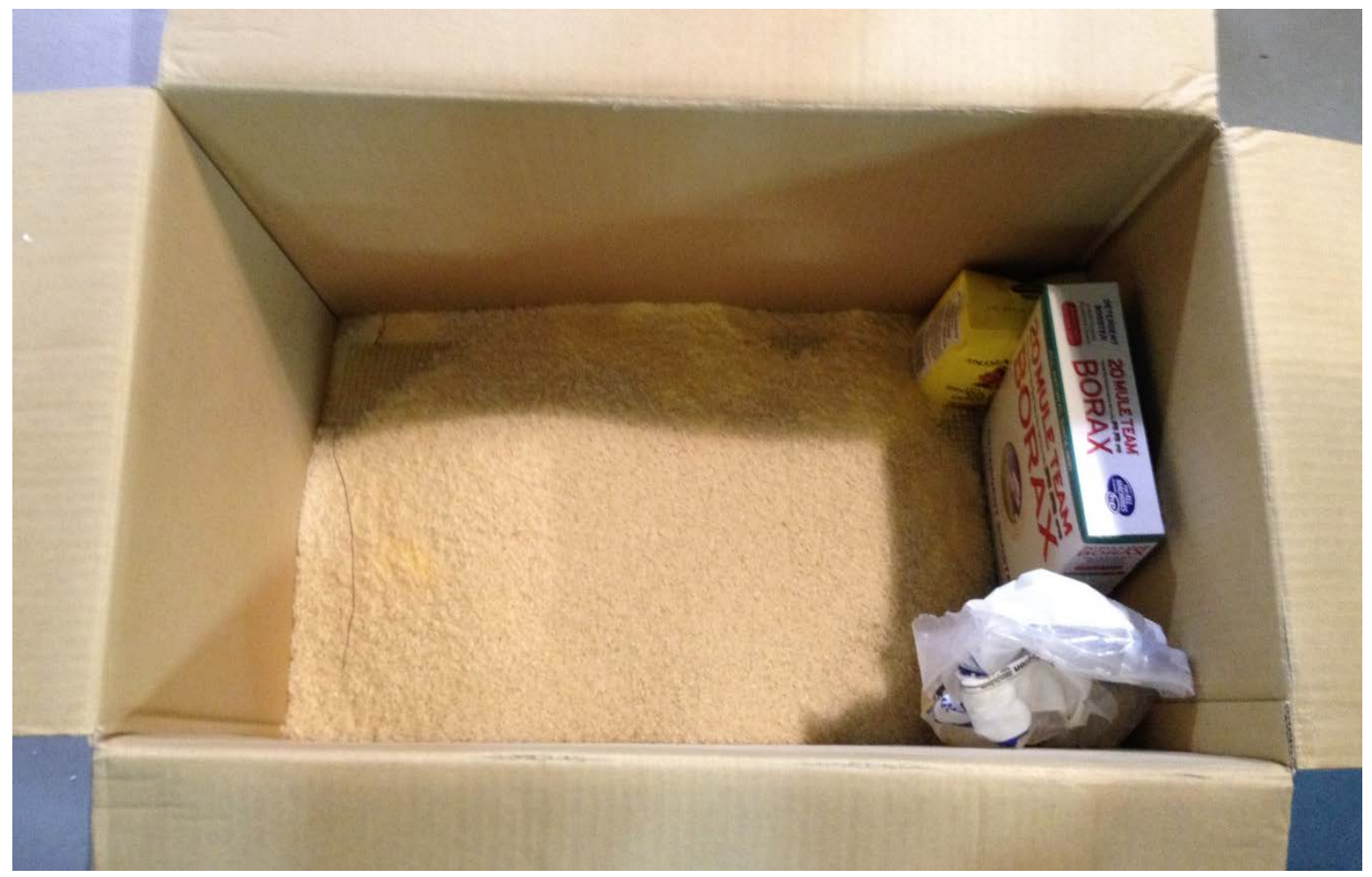

Figure 10: A Wing Covered in the Descant Mixture during the Preservation Process

The most important result of the preservation process is the rigidity of the final preserved wing model (W1) shown in Figure 11. The numerous bone joints became increasingly stiff even after the wing was removed from the desciants and placed in a climate controlled area. The skin, ligaments, and other tissue under the feathered surface also became very rigid. 


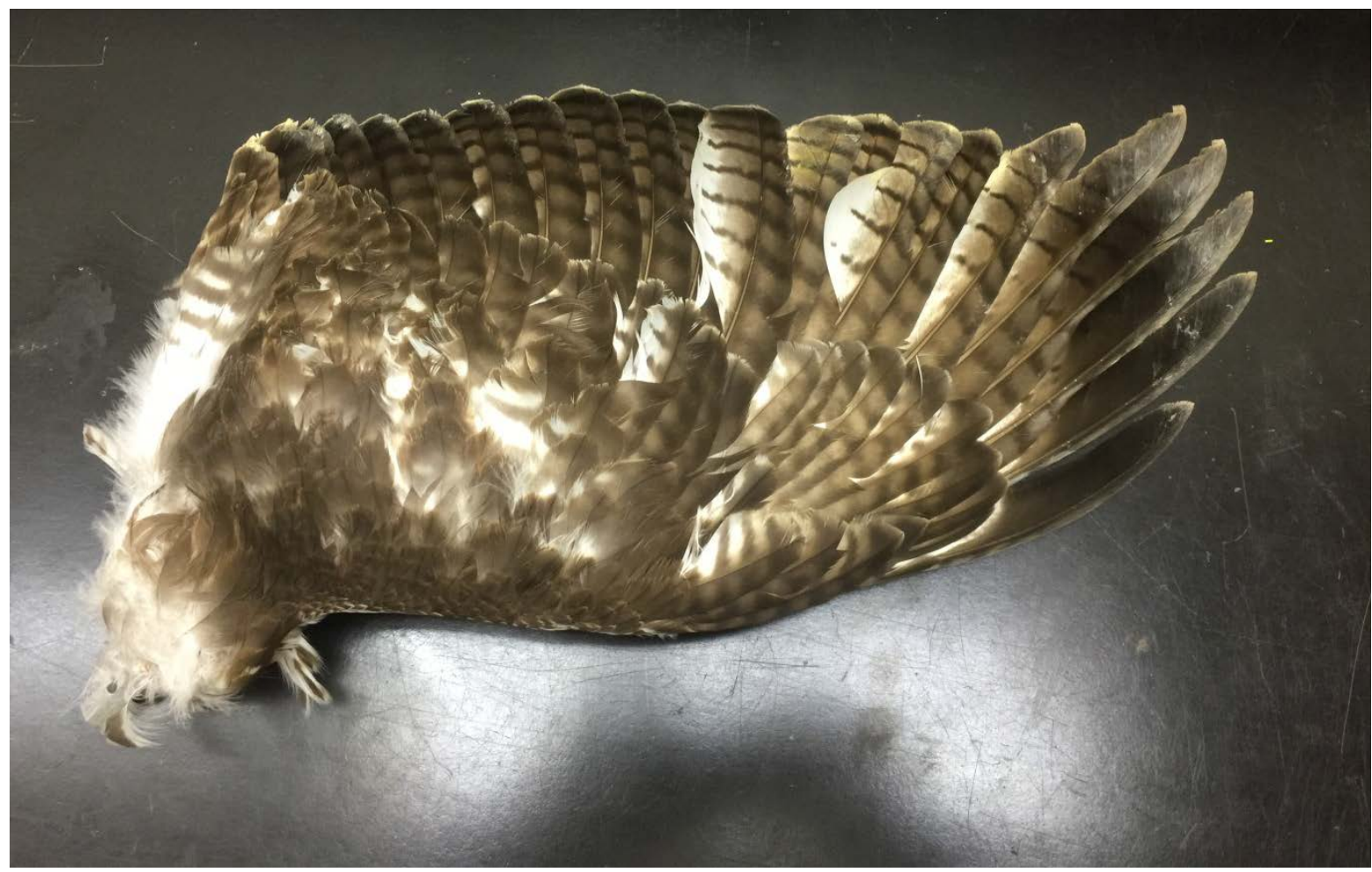

Figure 11: An Image of the Rigid and Sanitary Wing Resulting from the Preservation Process

Only a few negative results were noticed as effects of the preservation process. There was a slight reduction in the overall volume of the tissue under the feathers and skin due to the dehydrating the wing. This caused a slightly thinner build near the leading edge at the root of the wing. Preservation of the wing also caused the flesh underneath the feathers to feel stiff. This may have restricted the movement of the calamus or the portion of the feathers stem protruding from the flesh of the wing. However, these effects were acceptable and still allowed the primary objectives, to study the effects of feather motion and surface geometry, of this study to be completed.

Differences in the feathers before and after the preservation process were minimal. Feathers are not a living part of a bird and therefore, do not deteriorate at the same rate as flesh. 
They are also made of a protein called Keratin, similar to the finger nails [12]. As a result the feathers themselves did not noticeably differ from the feathers on newer donated specimen.

\subsection{Surface Applications to Reduce Feather Motion}

After the wing was preserved and aerodynamic force data was collected, three thick coats of Aqua Net hair spray were applied to the wing to reduce feather motion. Hair spray was chosen as the first surface application for several reasons. The first reason was that the hair spray would restrict, but not inhibit feather motion. Another reason hair spray was chosen, was the belief that it would not seriously alter the surface geometry of the wing. As seen in Figure 12, the hair spray was applied to the wing inside the wind tunnel and without disassembling any of the test equipment. This was done to prevent any changes in the mounting of the wing from occurring. 


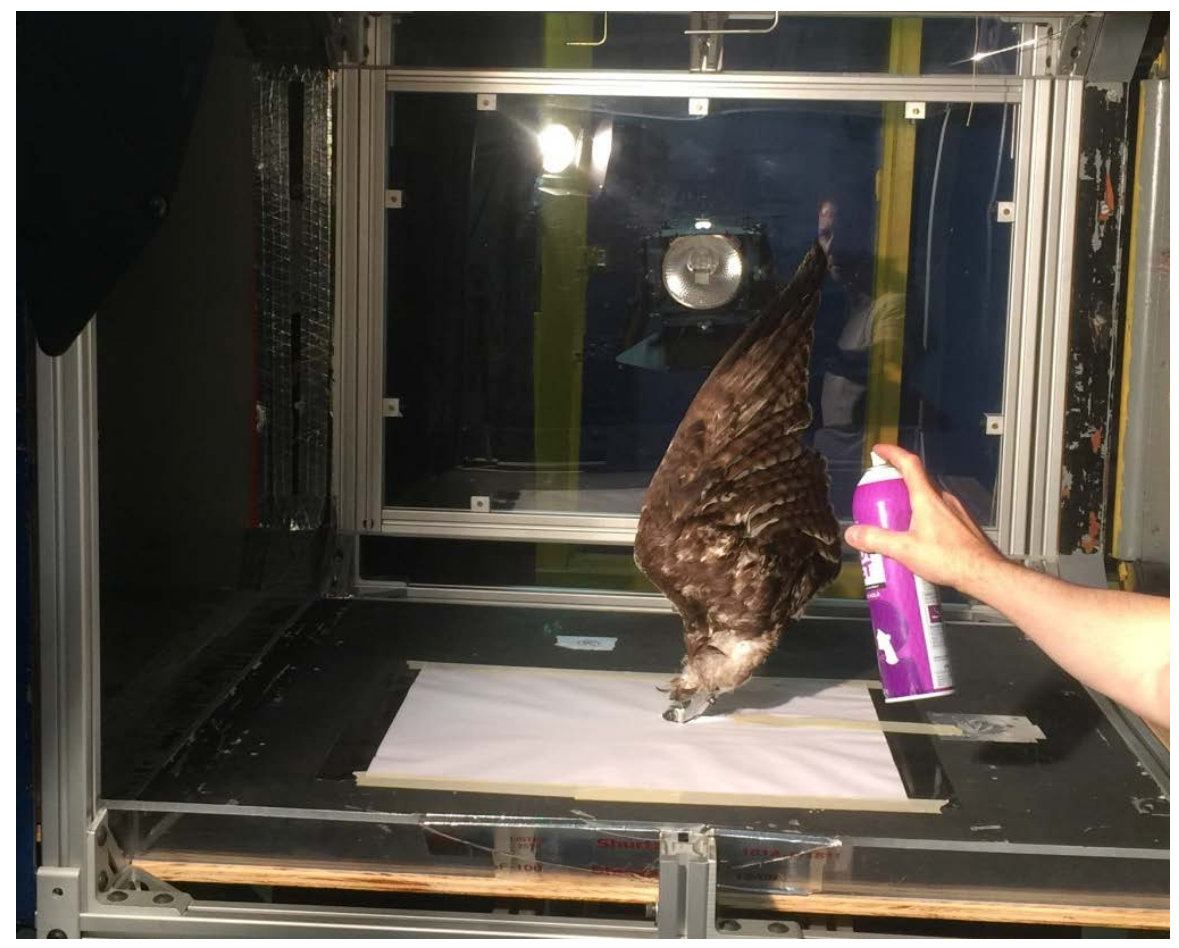

Figure 12: Hair Spray being applied to the Preserved Wing without Disassembling the Test Setup

After the required aerodynamic force data had been collected from the hair sprayed wing model (W2), the wing was ready for the next surface application to reduce feather motion. The next applicant was chosen to be Minwax Fast Drying Polyurethane. However, before polyurethane was applied to the wing, molds were taken of the wing.

It was believed that the polyurethane coating would severely alter the surface geometry of the wing. Therefore, molds of the wing were taken at this point. The blue residue seen on some feathers in Figure 13 is the result of the molding process. 


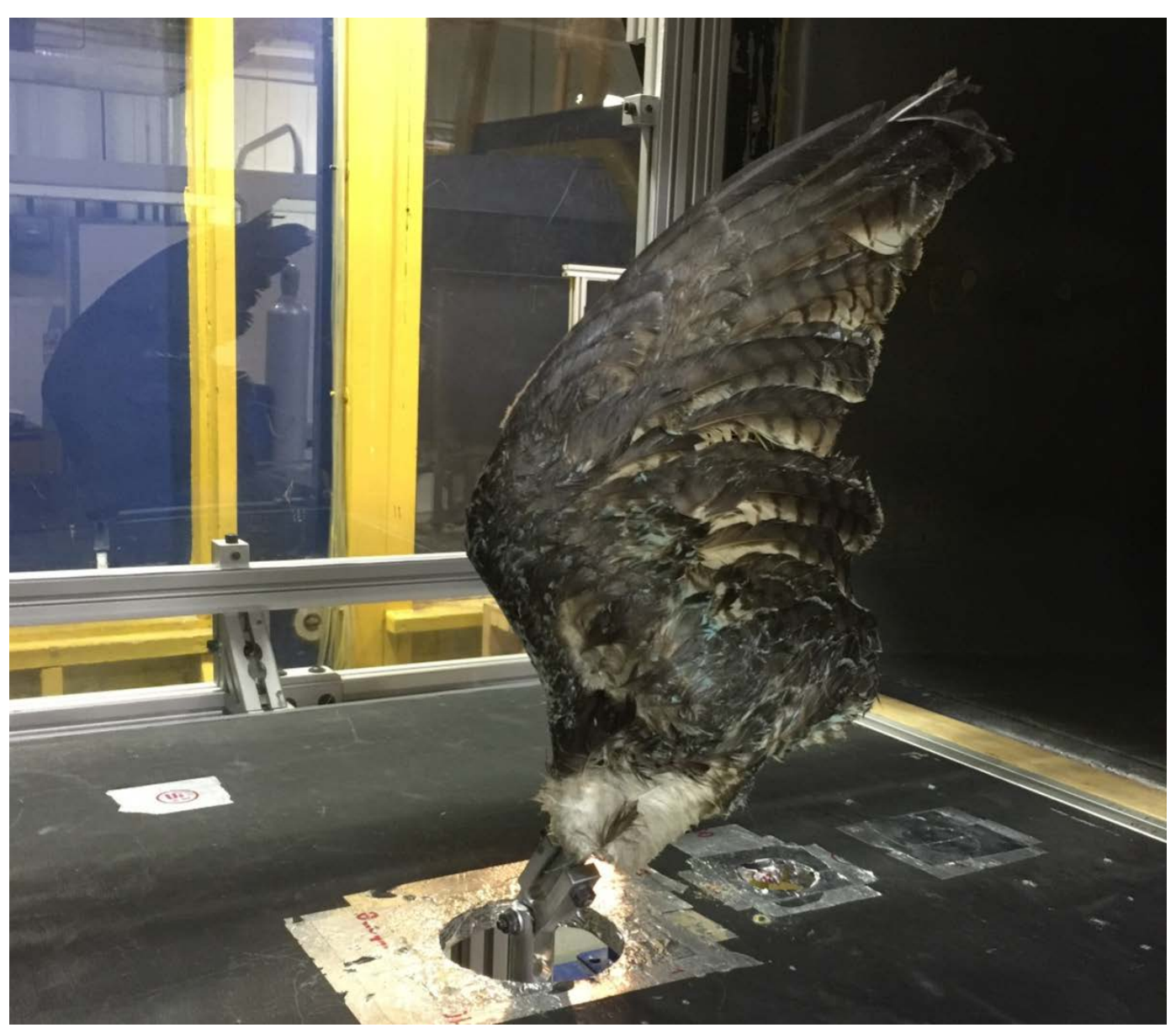

\section{Figure 13: The Polyurethane Wing Mounted for Testing in the Same Way as the W1 and W2}

Three coats of polyurethane were applied and allowed to dry before the wing was remounted in the WVU closed-loop wind tunnel. The wing was remounted in the test setup and aligned in the same orientation as W1 and W2. Alignment of the wing was checked using photographic documentation of the original set up. Aerodynamic force data was then collected from the polyurethane wing model (W3) in the same manner as the previous models. 


\subsection{Replication of the Natural Wing through a Mold and Cast Process}

\subsubsection{Development of the Mold and Cast Process}

Although very little feather motion was observed on W3, aerodynamic force data from a wing with no moving feathers was still required for comparison. It was determined; the best way to accomplish this was to reproduce a copy of the wing that still maintained the same macroscopic or overall shape and surface geometry. The final solution was to create a silicon rubber mold and polyurethane cast of the wing. However, a method to complete this task accurately needed to be developed.

Previous projects at WVU had used AeroMarine AM 125 Silicon Mold shown in Figure 14. It had been used to replicate items with surprising accuracy. Several bottles were still available. As a result, the residual supplies were the starting point for the development of the wing molding process. The development of the process began with determining if AM 125 could capture the microstructures of the feathers. However, it was soon noticed that hair sprayed feathers released from the dried mold much easier than natural feathers. The molds also degraded less due to easier removal of the feathers. 

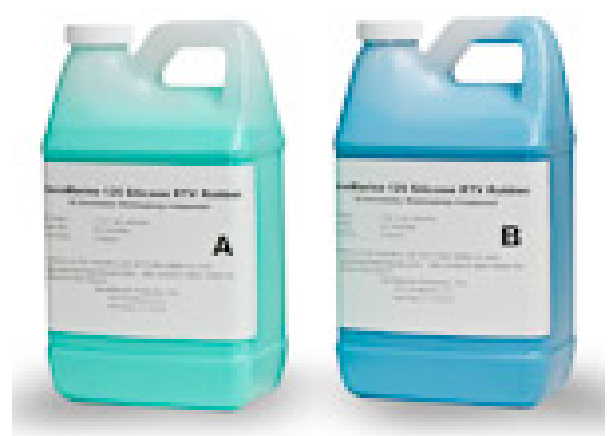

Figure 14: AeroMarine AM 125 Silicon Mold Used with W2 [21]

The significance of easier release was the improved condition of the mold and

feather after separation. Hair sprayed feathers left less feather particulate trapped in the mold, as well as a higher quality mold. Feathers that had been hair sprayed also took less AM 125 with them when they were removed. An example of these deficiencies can be seen in Figure 15. As the feather was removed from the mold, small pieces of the feather were left behind while small pieces of the mold were torn away in other areas. 


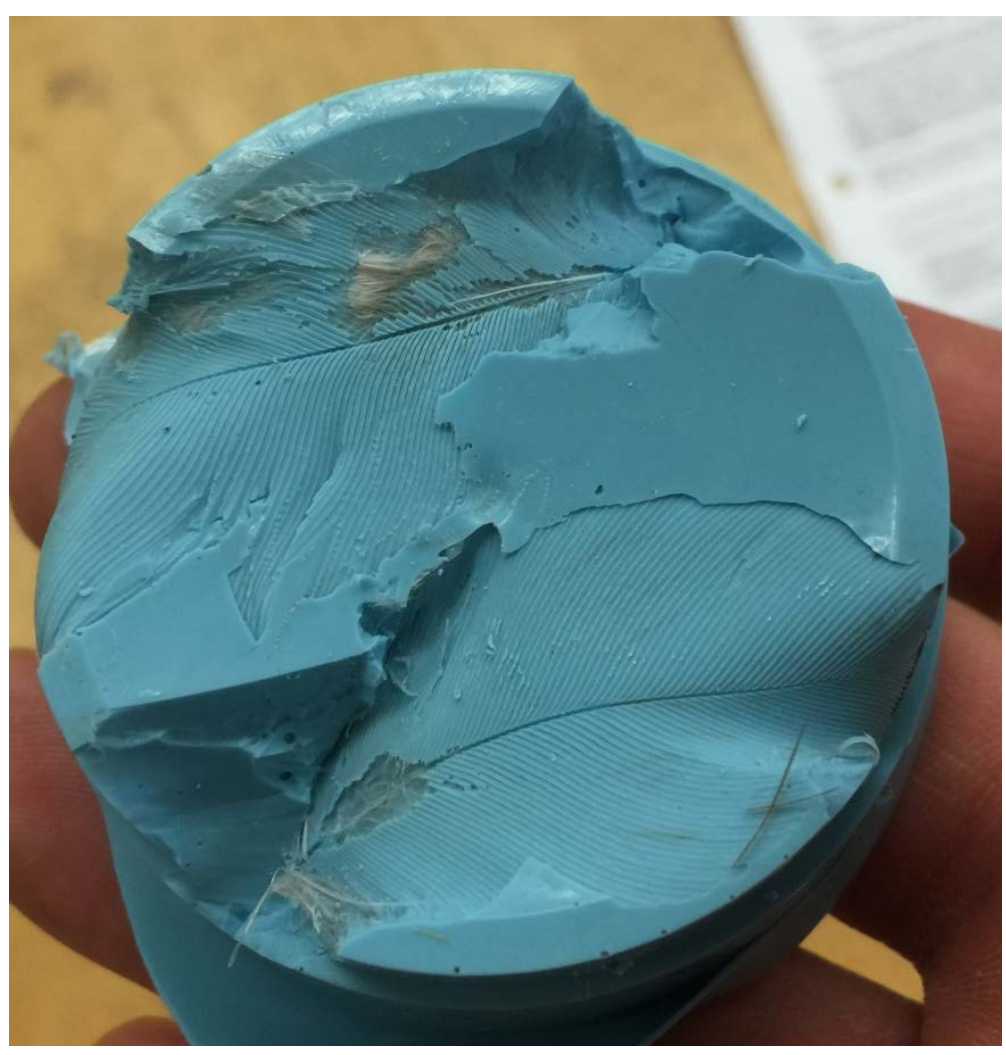

Figure 15: Wing Particulate Trapped in a Mold Created from an Untreated Feather

It was quickly determined that the first step was to find the best release agent reasonably available. This was accomplished by making small molds of individual feathers with different release agents applied. A comparison of the resulting molds is given in Table 1 and Table 2 below. 
Table 1: Feather Mold Release Agents and Their Test Results

\begin{tabular}{|c|c|c|c|c|}
\hline \multicolumn{5}{|c|}{ Feather Molding Test Matrix } \\
\hline \multicolumn{2}{|r|}{ Individual Feathers } & \multicolumn{3}{|c|}{ Results } \\
\hline Test & Release Agent & Ease of Release (\#/5) & Mold Quality & Notes \\
\hline 1 & Baby Powder & 4 & 4 & Smooth Release \\
\hline 2 & Petroleum Jelly & 5 & 0 & $\begin{array}{l}\text { Gaps in the mold and } \\
\text { clumping of the barbs }\end{array}$ \\
\hline 3 & Heated Petroleum Jelly & 5 & 1 & $\begin{array}{l}\text { Gaps in the mold and } \\
\text { clumping of the barbs }\end{array}$ \\
\hline 4 & Hair Spray & 3 & 4 & $\begin{array}{l}\text { Good mold quality } \\
\text { Acceptable release }\end{array}$ \\
\hline 5 & Soap + Isoprople Alcohol & 3 & 2 & $\begin{array}{c}\text { Some clumping of } \\
\text { the barbs }\end{array}$ \\
\hline 6 & Hair Spray + Petroleum Jelly & 5 & 1 & $\begin{array}{l}\text { Gaps in the mold and } \\
\text { clumping of the barbs }\end{array}$ \\
\hline 7 & $\begin{array}{c}\text { Hair Spray + Petroleum Jelly } \\
\text { Heated }\end{array}$ & 5 & 0 & $\begin{array}{c}\text { Gaps in the mold and } \\
\text { noticable clumping of } \\
\text { the barbs }\end{array}$ \\
\hline 8 & $\begin{array}{c}\text { Hair Spray + Soap + Isopropyl } \\
\text { Alcohol }\end{array}$ & 4 & 4 & $\begin{array}{l}\text { Small bubbles visible } \\
\text { in the mold, } \\
\text { possibly from alcohol } \\
\text { evaportation }\end{array}$ \\
\hline 9 & Hair Spray + Baby Powder & 4 & 5 & $\begin{array}{c}\text { Very good, minimal } \\
\text { damage to mold. } \\
\text { Mold is reusable }\end{array}$ \\
\hline
\end{tabular}

From the results shown in Tables 1 and 2, the combination of the same Aqua Net hair spray used to prepare W2 and baby powder performed the best. However, this was for single feather applications. The next step was to determine the best release agent for several feathers together as on an actual wing. 


\section{Table 2: Photo Documentation of Release Agent Test Results}

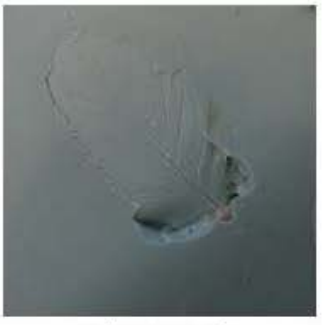

Baby Powder

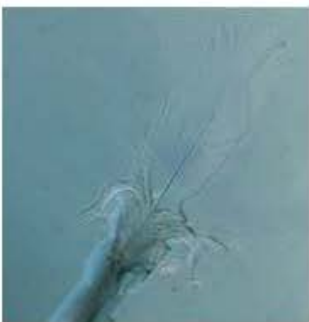

Hair Spray + Soap + Isopropyl Alcohol

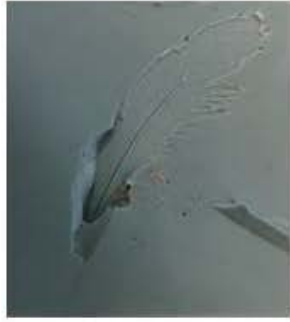

Hair Spray

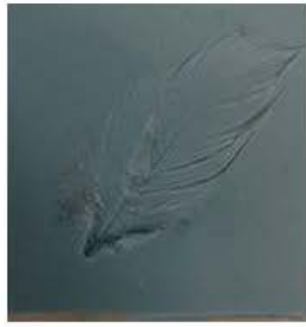

Hair Spray + Isopropyl Alcohol

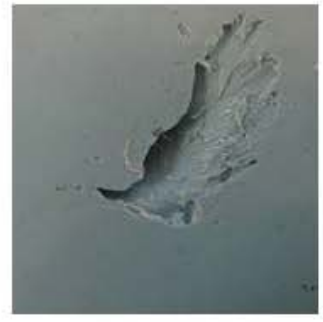

Petroleum Jelly

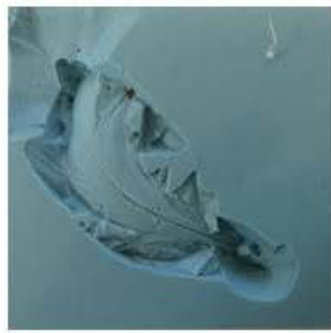

Hair Spray +

Petroleum Jelly

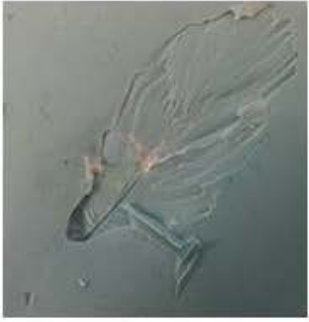

Heated Petroleum Jelly

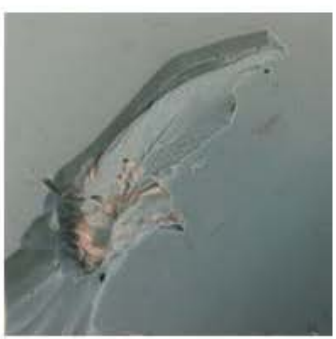

Hair Spray + Heated Petroleum Jelly

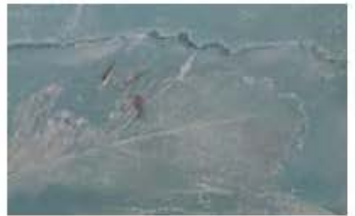

Hair Spray + Baby Powder

In order to further test the release agents, two part molds were made of two groups of feathers using the three most promising release agents used for the individual feather mold tests. Casts were then made from each mold using AeroMarine Polymer Casting Resin. The same release agent was also used in the preparation of the mold for casting. After completion, the resulting casts, shown in Figure 16, were then compared to determine the best release agent. 

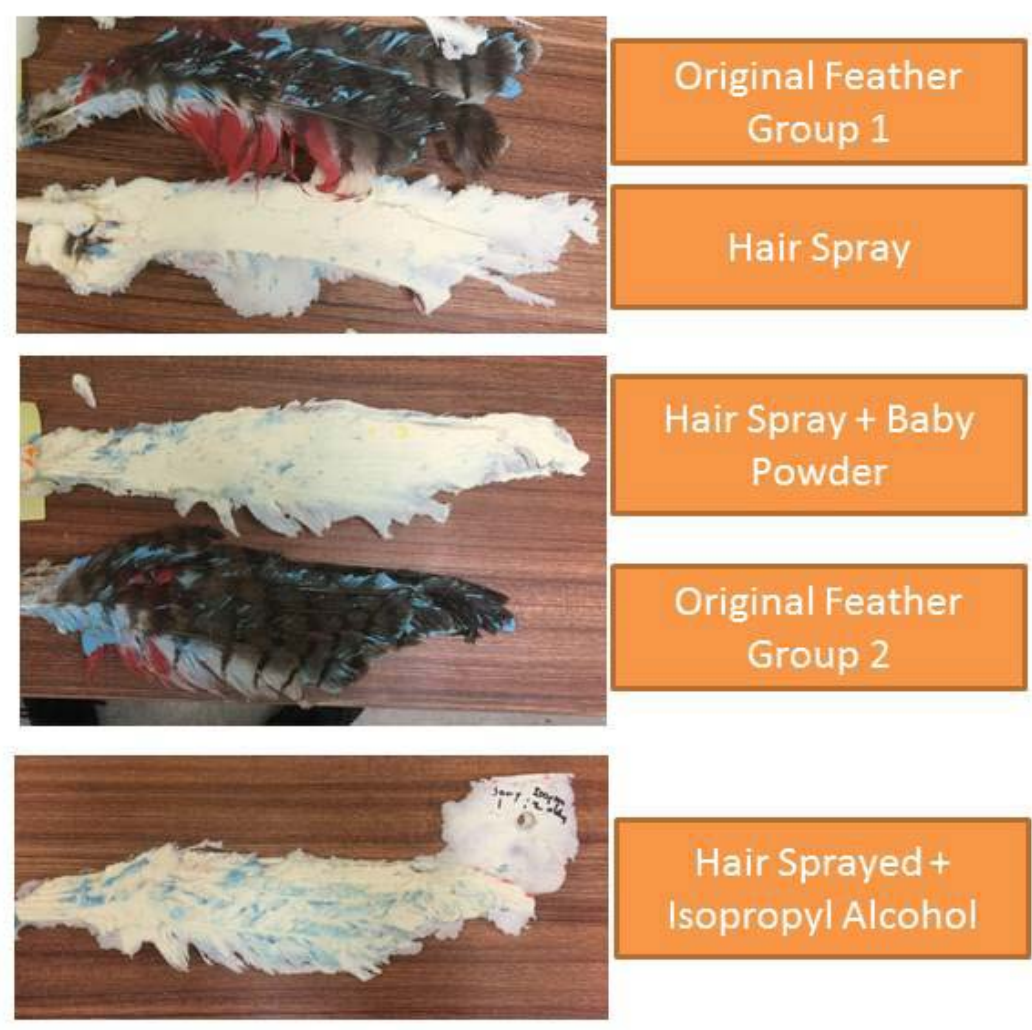

Hair Sprayed +

Isopropyl Alcohol

\section{Figure 16: Test Casts of Feather Groups Using Different Release Agents}

The resulting casts shown in Figure 16 help illustrate the test results. Although hairspray released fairly well, it did not reproduce thinner sections of the feather. The combination of isopropyl alcohol and hair spray provided the best reproduction of the entire feather group. However, removing the cast from the mold damaged parts of the mold by taking the cured silicon with it.

As a result, the combination of hair spray and baby powder was chosen to be used in the production of a full wing cast. This combination was less proficient at reproducing thin sections of the feathers. However, the resulting cast released much easier and without causing as much damage to the mold. Therefore, it allowed more casts of similar quality to be made from the same mold. 


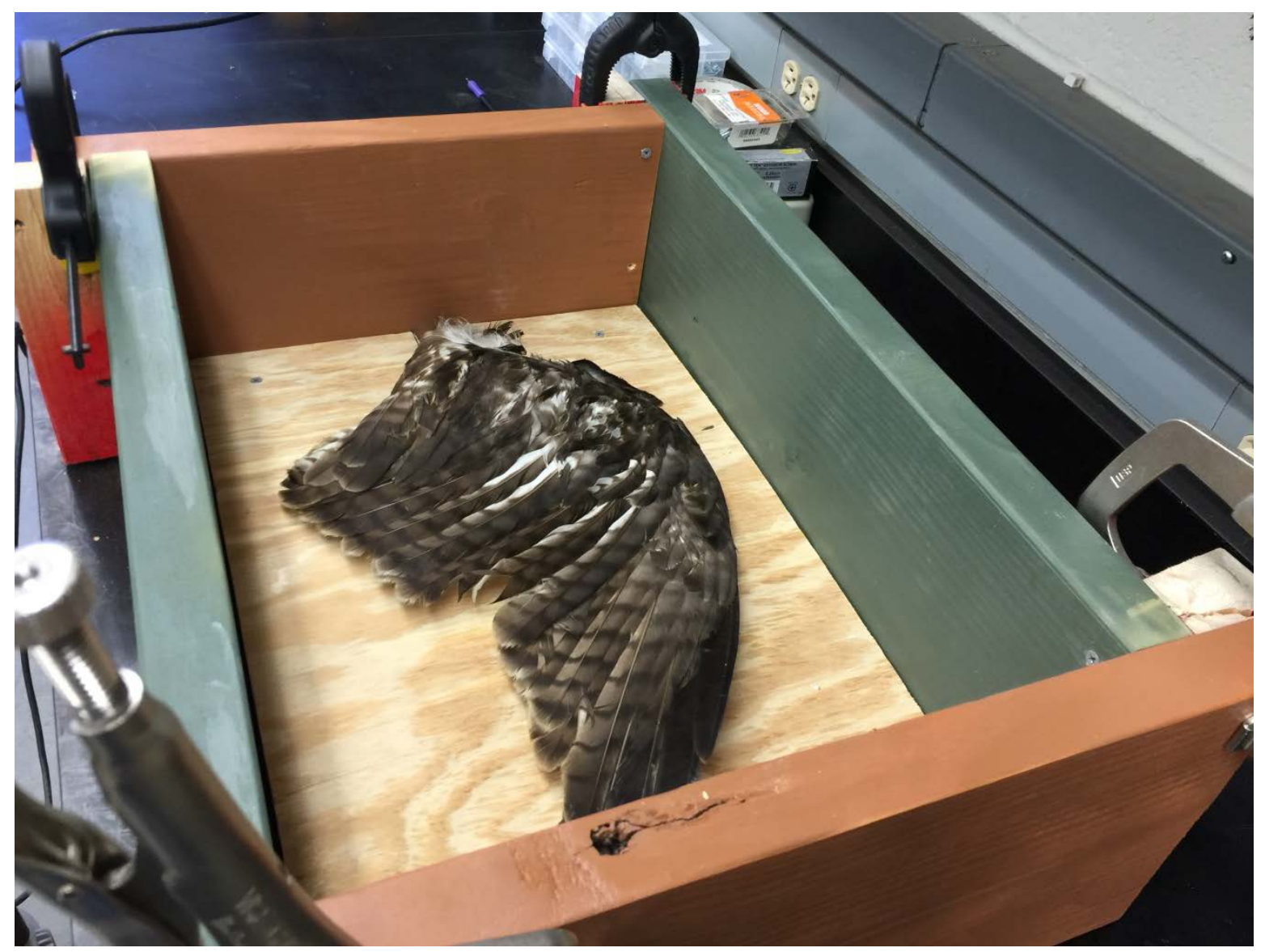

Figure 17: The first Stages of Preparing W2 for Molding in the Mold Box

After the best methods and materials were determined, the final process was put to the test. In order to create a mold of the entire wing, a 25” W x 15” $\mathrm{H}$ x 8” D mold box was built. The box was made using ten inch wide pine boards. The boards were painted using Krylon spray paint. The layer of paint served to seal the wood and prevent AM 125 or polyurethane resin from bonding with the wood during the process. 


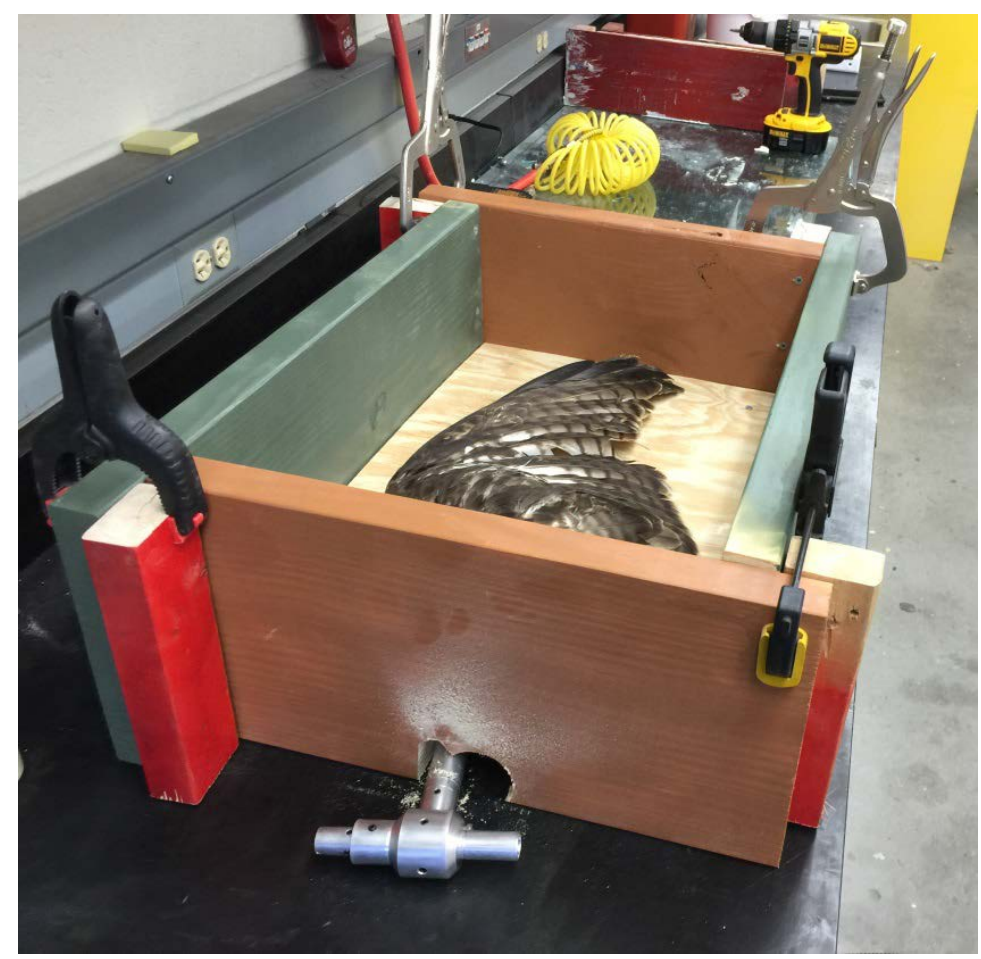

Figure 18: L Shaped Corners and Clamps used to maintain the Mold Box's Dimensions

2” x 4” boards were cut to length as needed and attached to one of each board. A clamp was then applied to the excess board of one side and the 2 ” $x 4$ ” portion of the adjacent board. Using this design, the sides of the mold box could be quickly removed and reattached as needed. This design was also used to accommodate future models with varying dimensions. 


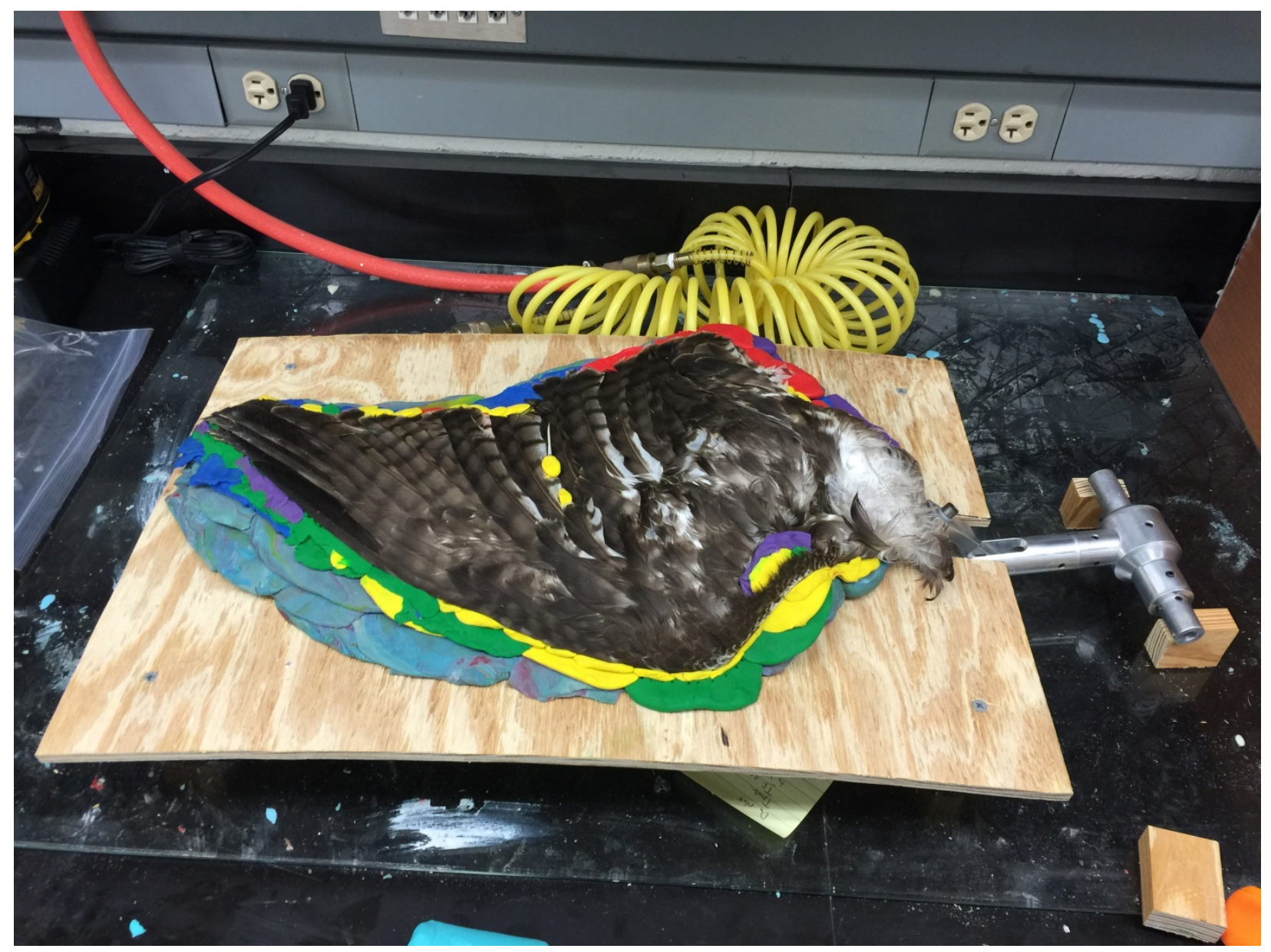

Figure 19: Modeling Clay and Play Doh used to Seal the Parting Plain of the Mold

After the mold box had been sized, it was removed to make the process of creating a barrier along the edges of the wing. This barrier or seal was to ensure only the AM 125 remained on one side of the wing. Modeling clay and Play Doh were used to form the barrier. Both materials were easily pliable and could be tightly formed to the shape of the wing.

Once a suitable barrier was created, the mold box was rebuilt around the wing. Modeling clay and Play Doh were used to seal the gap between the board serving as the bottom barrier and the mold box walls. In order to reduce the amount of AM 125 required, small boards and pieces of metal were used to prevent AM 125 from flowing into corners and other unused areas of the 
box. Metal acorn nuts were positioned a few inches from the wing around the box. The use of these nuts created keys for the two parts of the mold to be realigned during casting.

After the container had been prepared, the wing was coated with hairspray and baby powder to serve as a release agent. AM 125 was then mixed according to AeroMarine instructions and poured over the wing. Approximately one gallon of AM 125 was used to provide a half inch minimum depth on all parts of the wing.

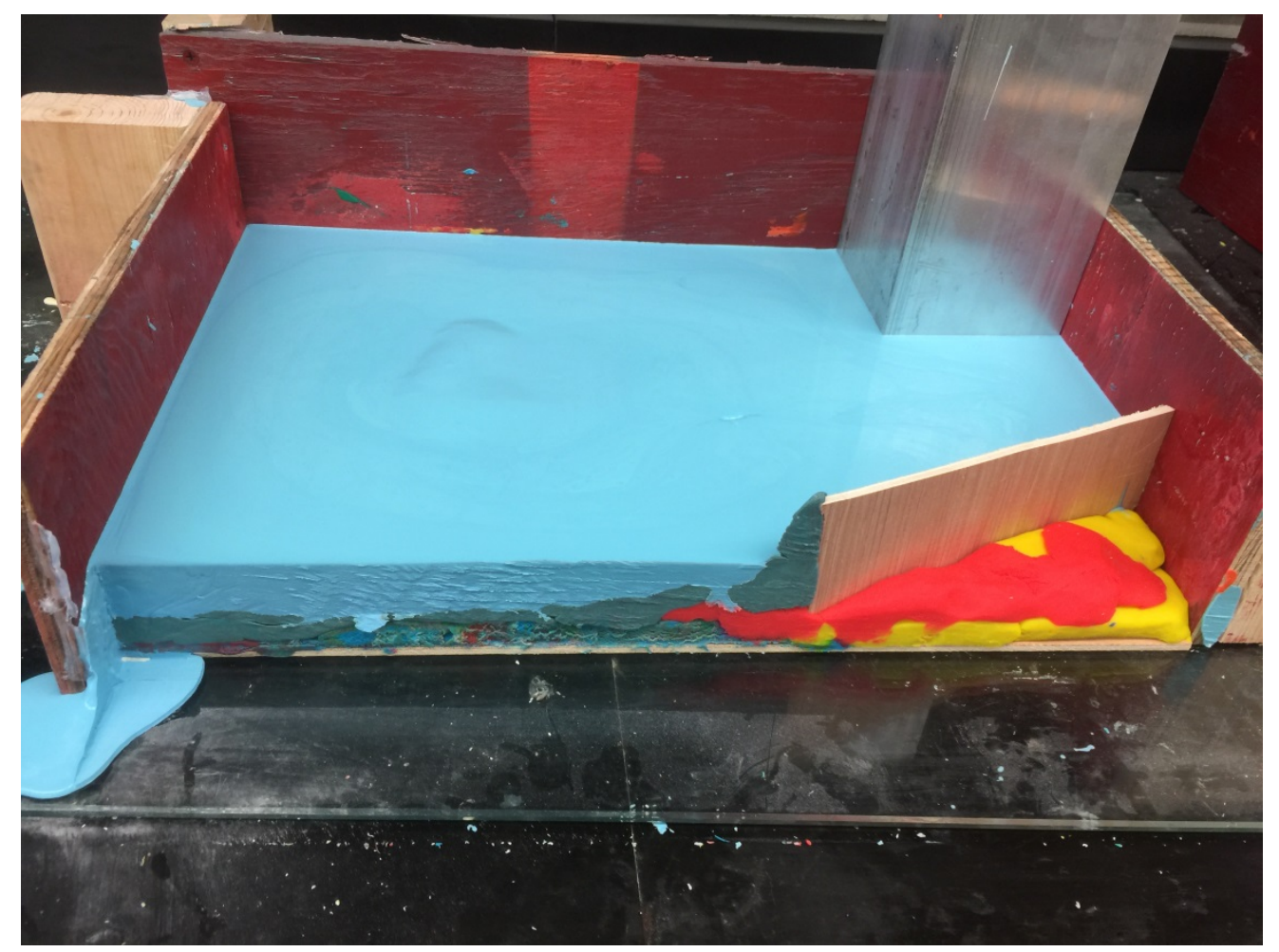

Figure 20: The First Part of the Two Part Mold during Removal from the Mold Box

The AM 125 was allowed to cure for twenty four hours. After this period, the mold was gently removed from the box by removing the walls as seen in Figure 20. However, the wing was not separated from the AM 125. The acorn nuts were removed at this point. 
The wing was flipped to the opposite side. AeroMarine Eject It was applied to the surface of the first part of the mold. Eject It was recommended by AeroMarine to prevent the two halves of the mold from adhering [21]. The mold box was then rebuilt around the wing. Modeling clay and Play Doh were used as needed to seal off unused areas of the box. Hairspray and baby powder were lightly applied to the wing. AM 125 was then mixed and poured over the wing as seen in Figure 21.

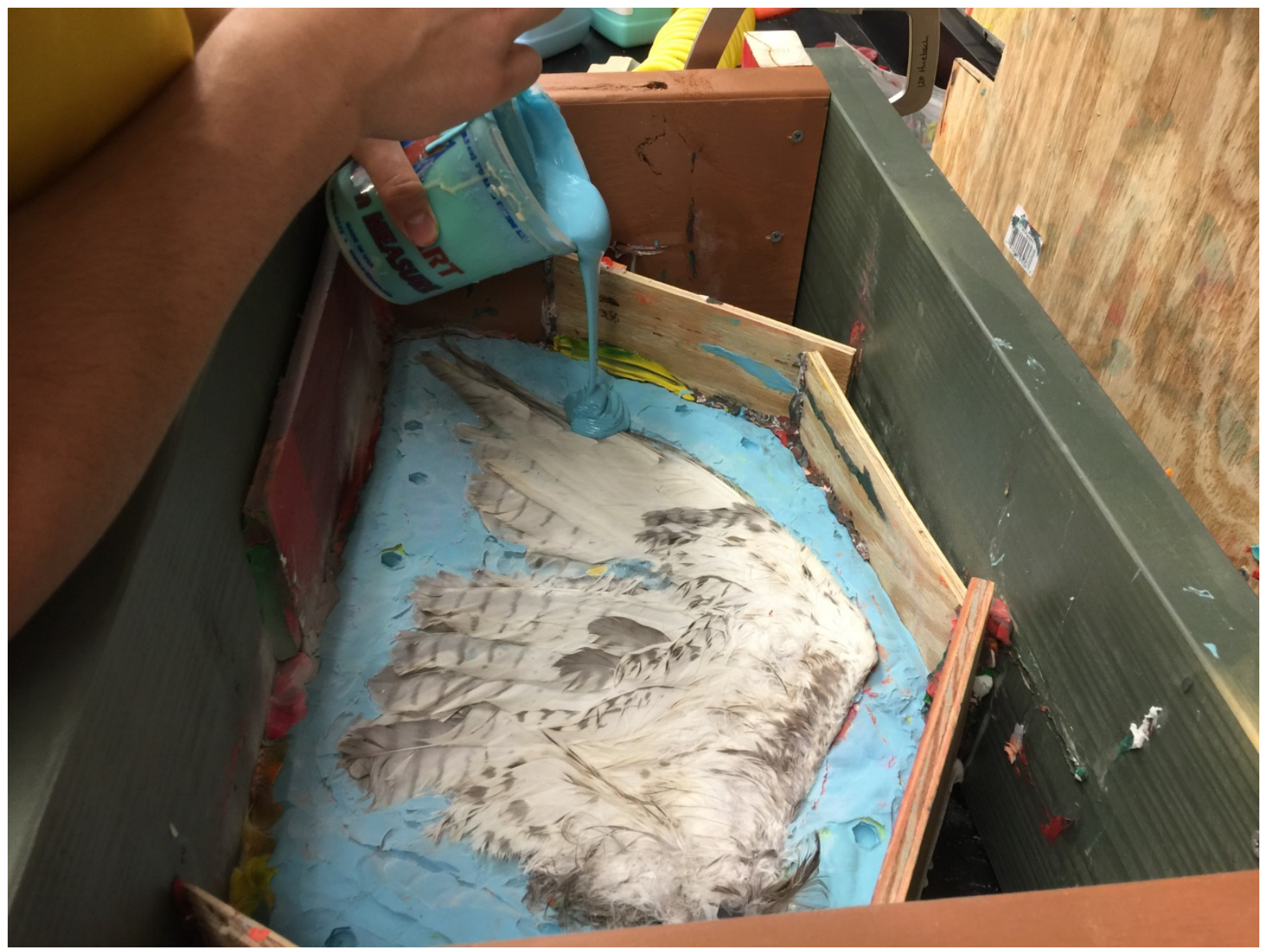

Figure 21: Pouring the Second Part of the Mold onto the Prepared Wing

After the second part of the mold had cured for twenty four hours, the mold box was disassembled. The first half of the mold was separated from the wing through a delicate and 
time consuming process. Even with the use of a release agent, special care must be used to remove the mold by gently pulling the mold away with the grain of the wing. Once the first half of the mold had been removed, the shape and surface texture of the mold cavity were revealed as seen in Figure 22. Some feathers were trapped in the mold. These were removed without damaging the mold wherever possible using tweezers.

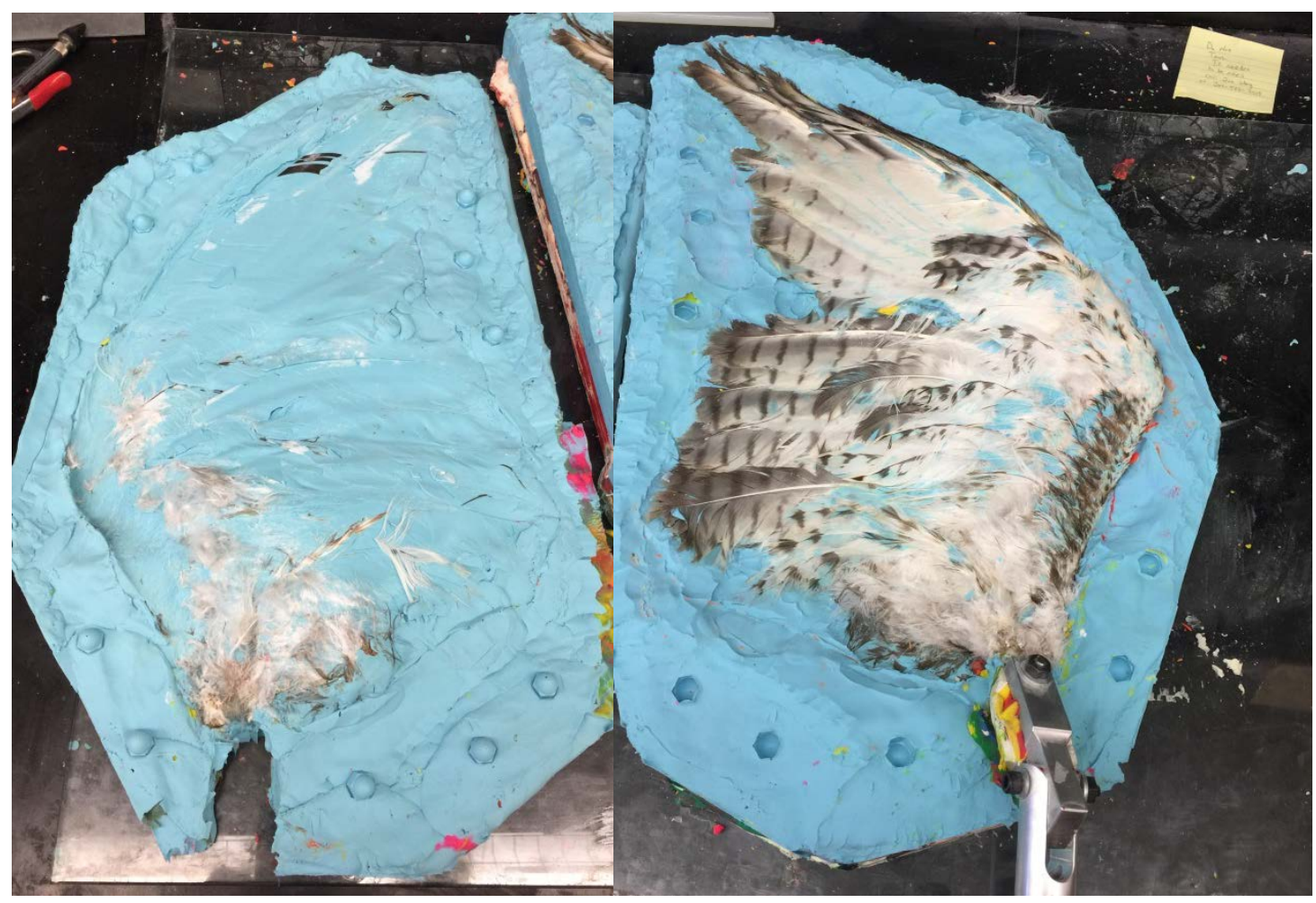

Figure 22: The Completed Two Part Wing Mold 
After both halves of the mold had been separated from the wing, and feather residue had been removed, the mold was prepared casting. Baby Powder was brushed onto any part of the mold that was expected to come in contact with the casting resin as seen in Figure 23. Hair Spray was then lightly applied to the same surfaces.

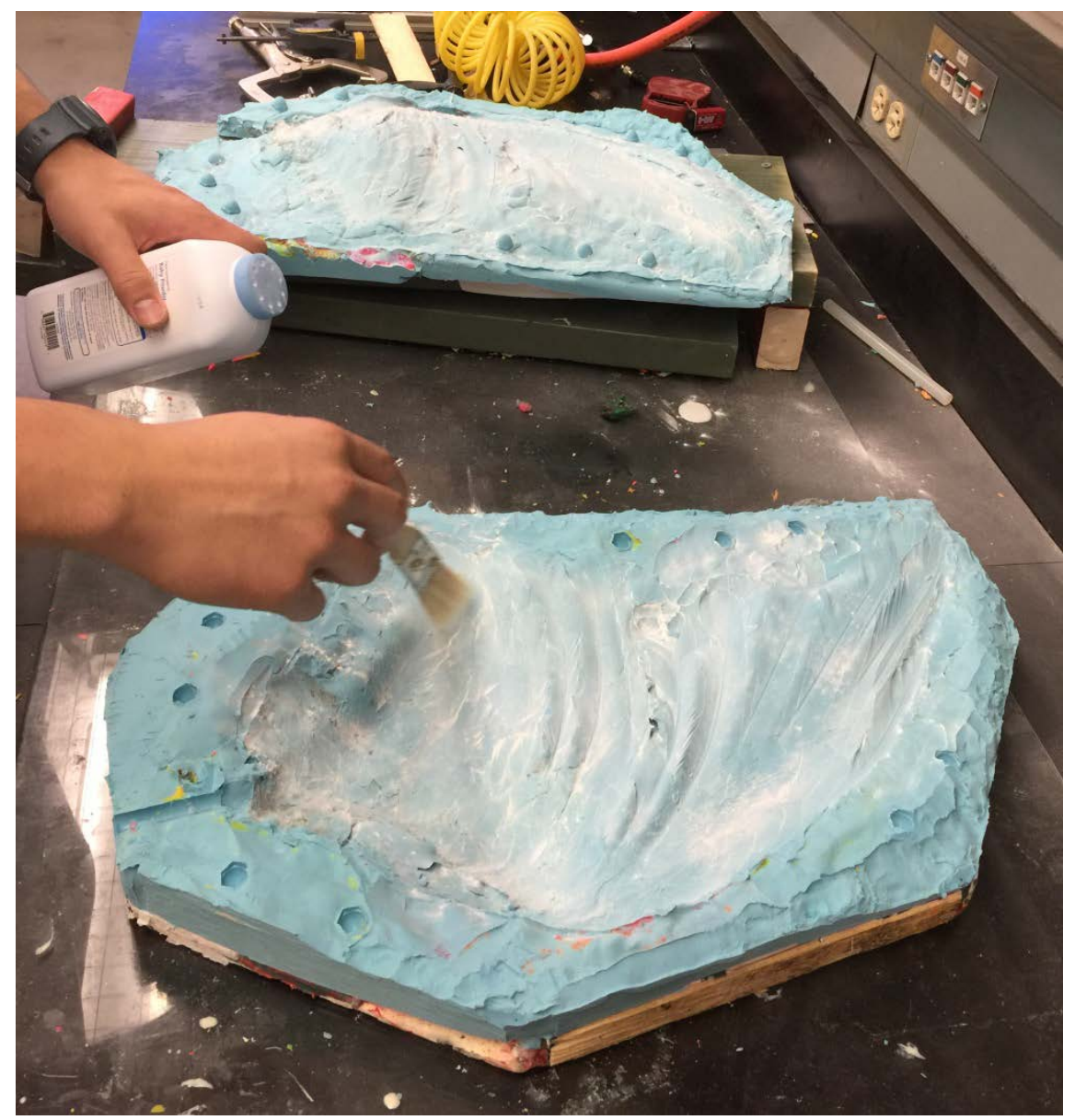

Figure 23: Preparing the Two Part Mold for Casting

Two walls from the mold box were secured on either side using a common high strength temporary adhesive material, duct tape. Duct tape was wrapped around the mold to provide the compressive force needed to seal the mold. Figure 24 shows the mold once it had been stood upright and secured. AeroMarine Polymer Casting Resin was mixed and poured into the mold 
half a quart at a time until the mold was full. Casting resin was then mixed and added as needed to replace material leaking from the mold for the first few minutes. This material cures quickly and soon sealed the mold leaks.

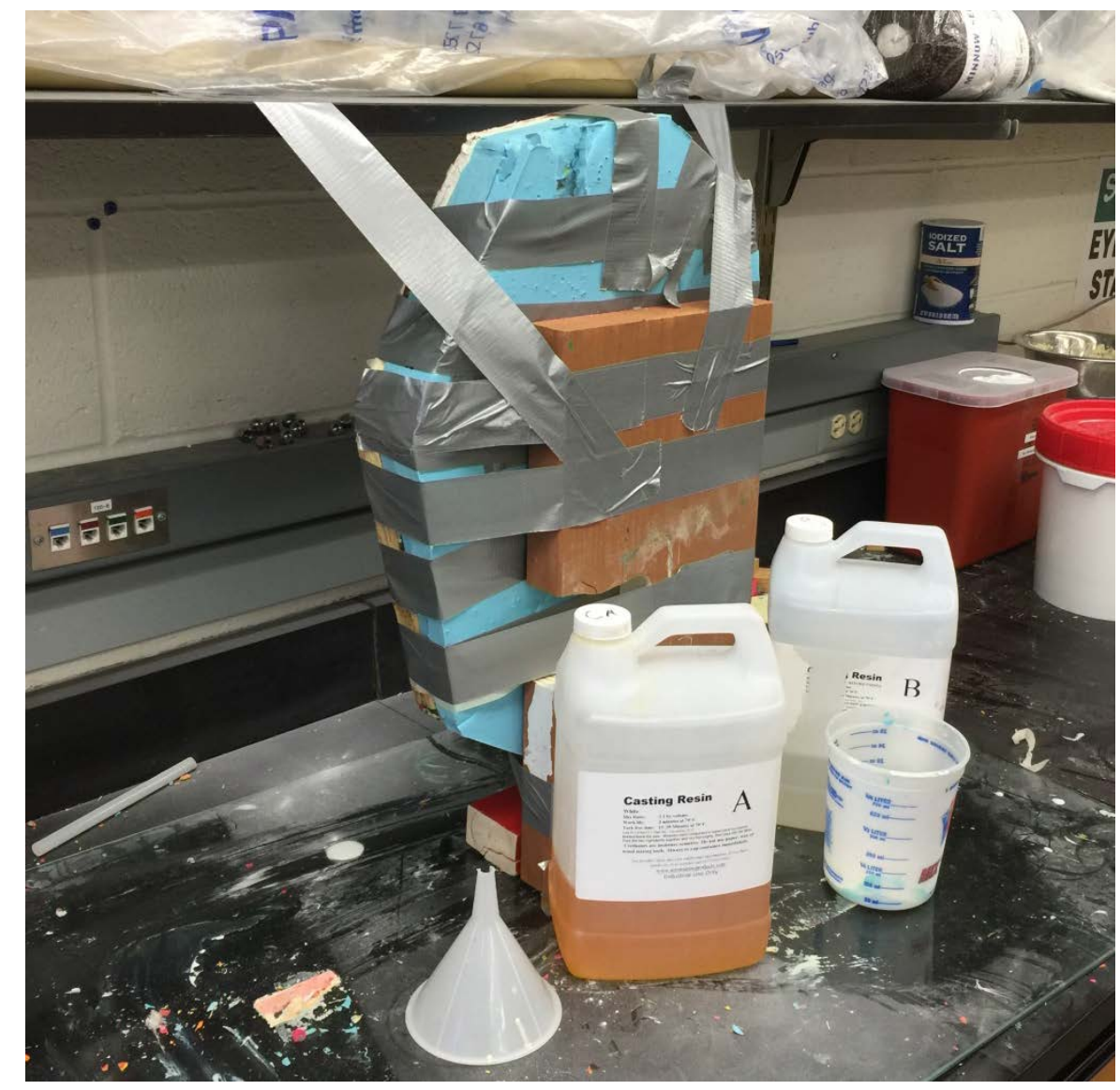

Figure 24: The Wing Mold Ready for the Casting Resin to be Poured

The casting resin was allowed to cure for twenty four hours. After this period had elapsed, the first part of the mold was removed revealing the model shown in Figure 25. The same care used to separate the mold from the wing was also required to separate the cast wing model (W4) from the mold. If done incorrectly, the mold would have been seriously damaged. 


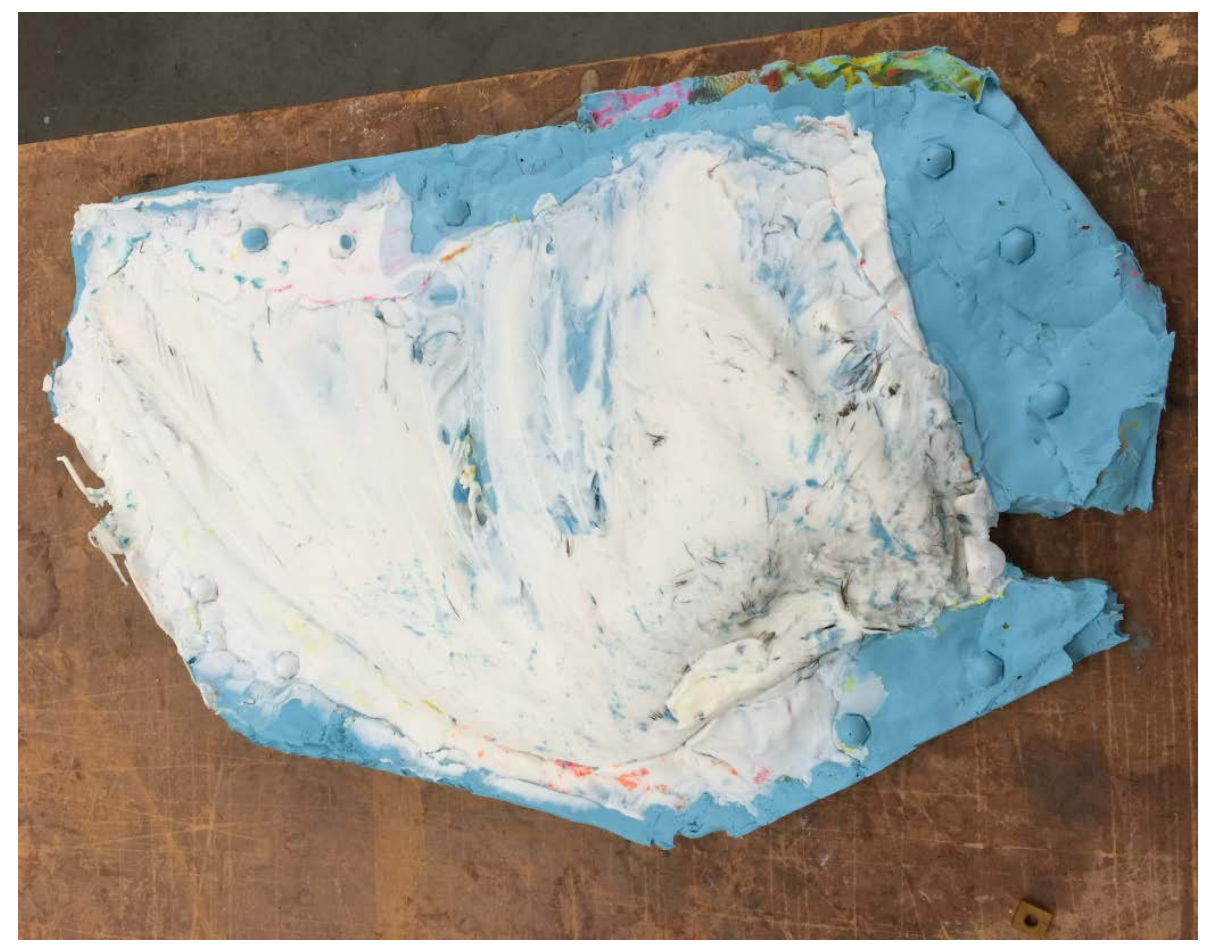

Figure 25: The First Look at the Cast Wing Model

\subsubsection{Preparation of the Cast Wing Model for Testing}

As seen in Figure 26, the cast wing resulting from the mold and cast process still required work before it was to be used for testing. Excess resin protruded from both the leading and trailing edges of the wing. Small chunks AM 125 were struck the surface of the cast. 


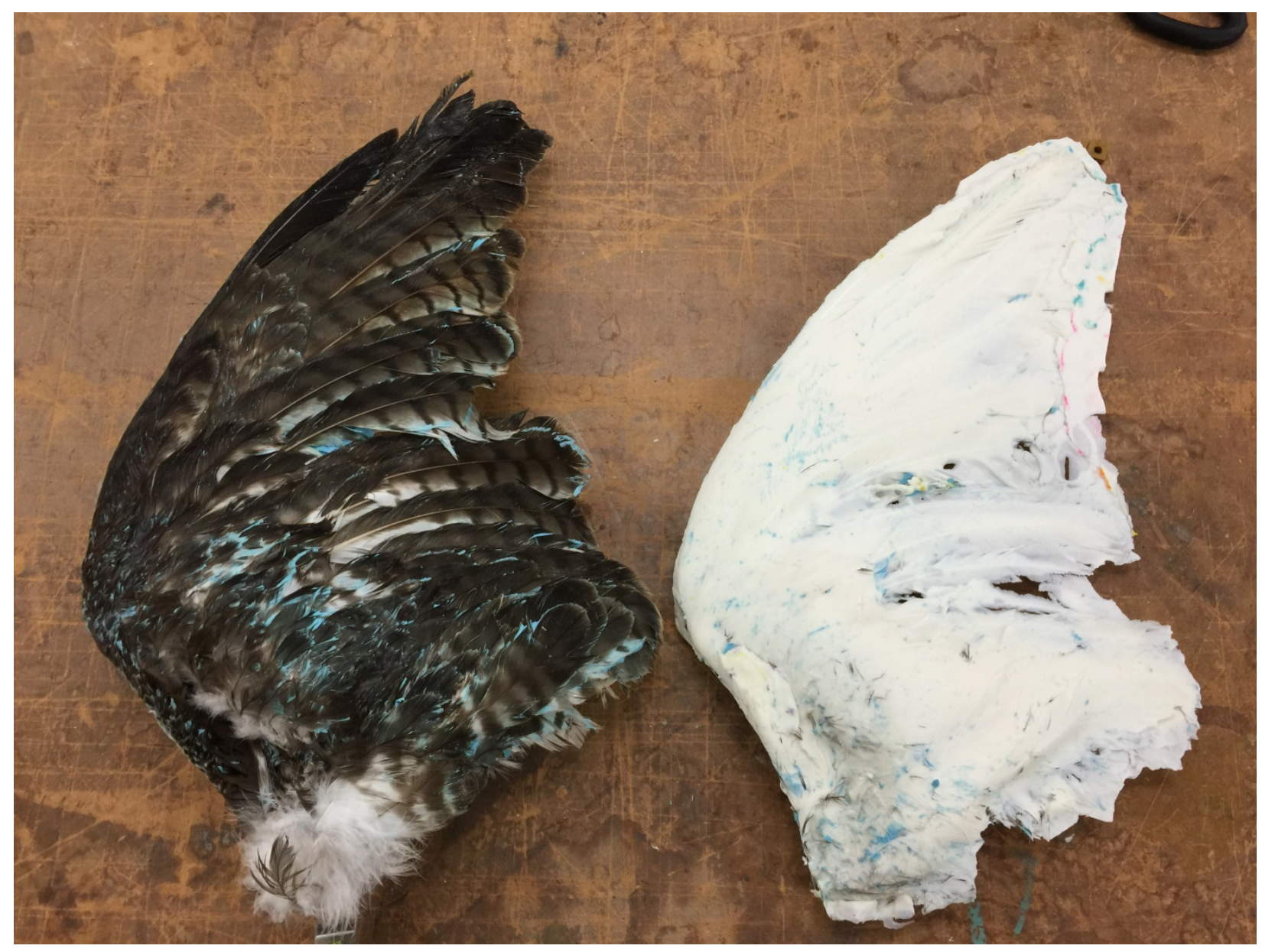

Figure 26: An Early Comparison of the Original and Cast Wings

In order to finish the wing model, a Dremel rotary tool was used to file away the excess casting resin along the wing perimeter. The leading and trailing edges were also sanded smooth and shaped to resemble W2. Tweezers were used to remove as much residual AM 125 as possible. 
Once W4 was shaped, a hole was drilled and threaded at the base of the model. This allowed an aluminum mounting fixture to be attached in the same location as on the original wing. W4, seen in Figure 27, was then tested in the WVU closed-loop wind tunnel using the same method as W1, W2, and W3.

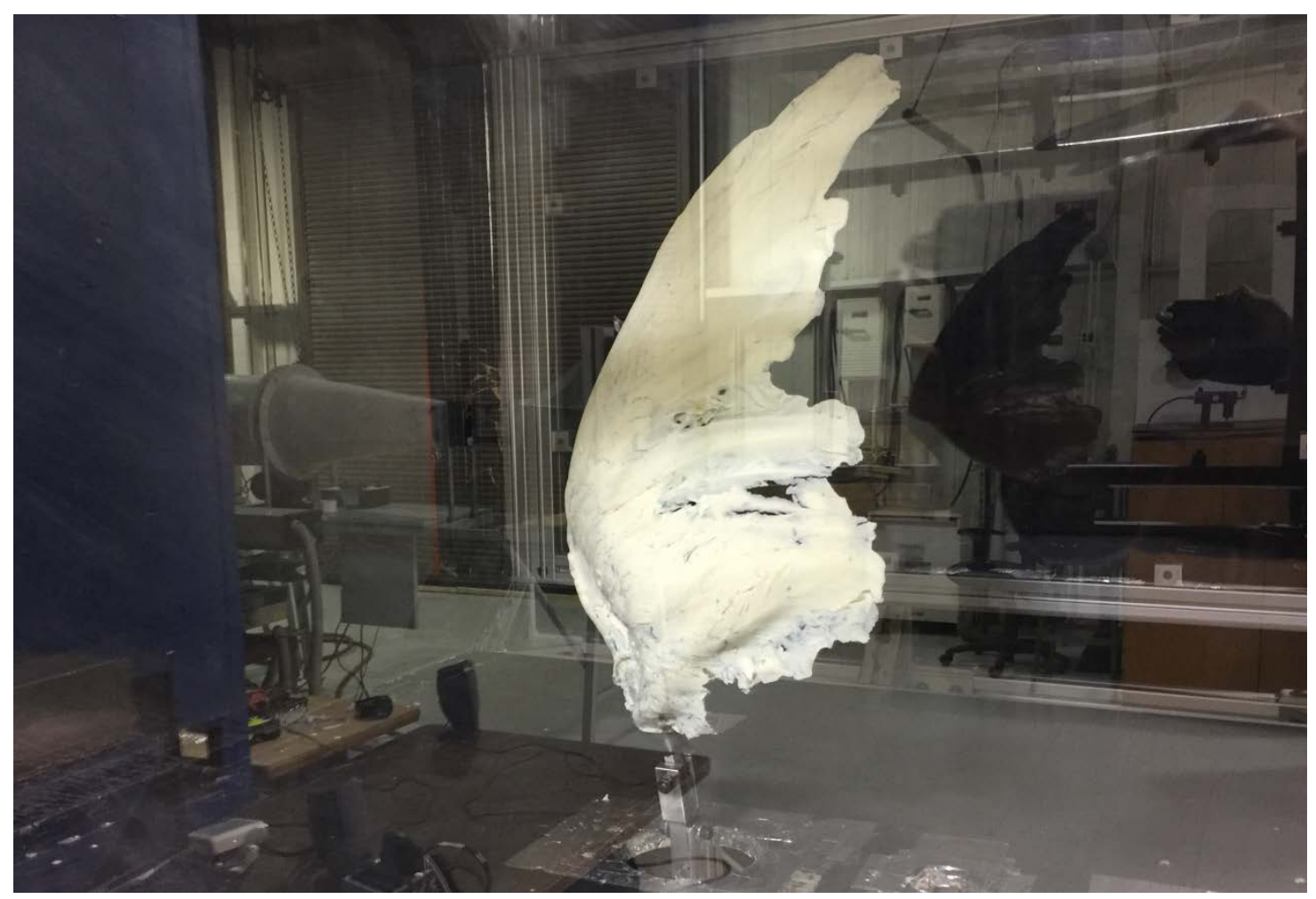

Figure 27: The Final Cast Wing Model Prepared for Testing 


\subsection{Application to Reduce Surface Geometry of the Cast Wing}

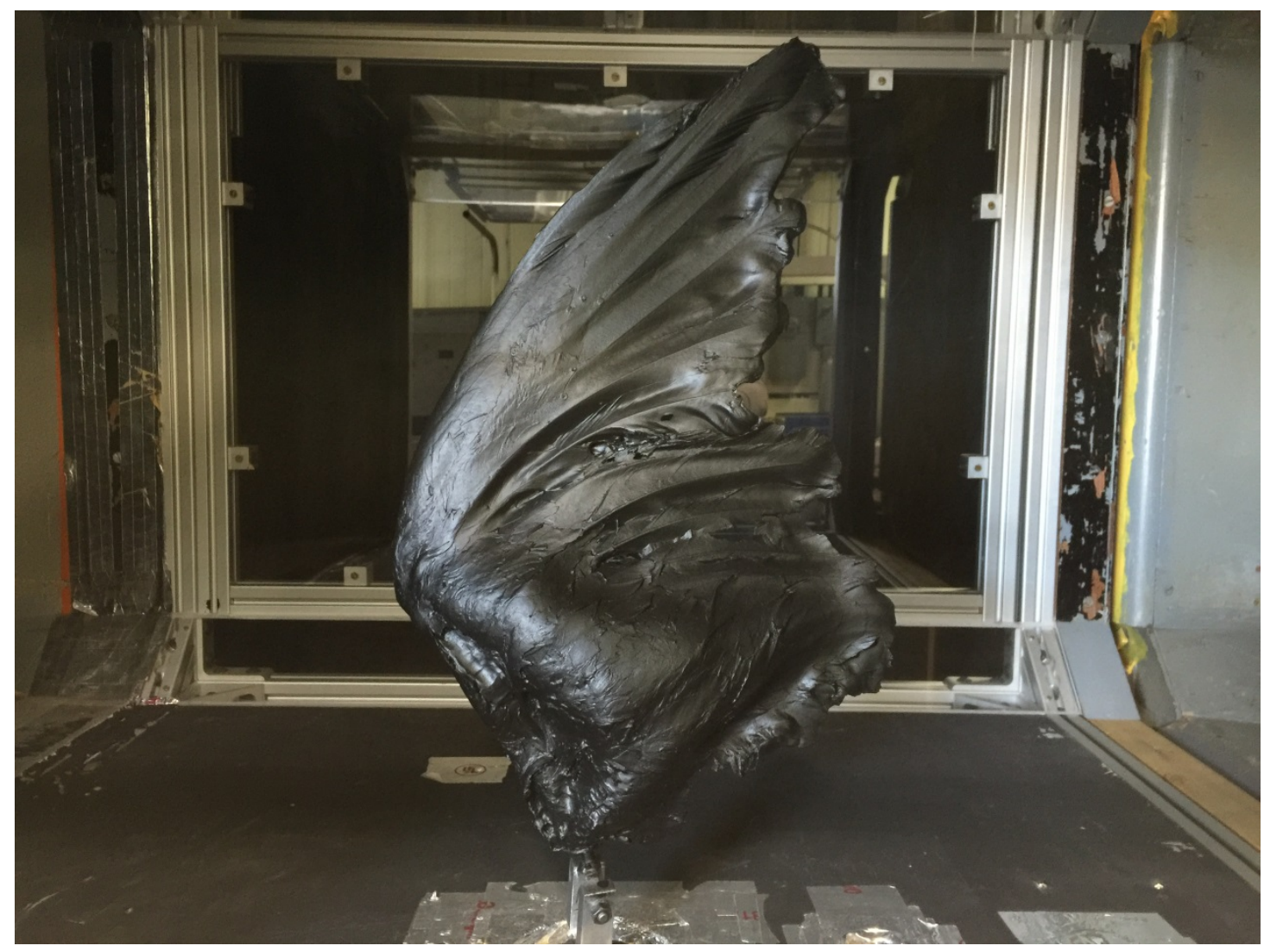

Figure 28: The Cast Wing Model with a Thick Coat of Plasti Dip to Reduce the Surface Geometry

After the required aerodynamic force data had been collected from W4, the wing was coated with Performix Plasti Dip Multi-Purpose Rubber Coating. Applying Plasti Dip to the surface of the wing reduced the surface geometry of the cast wing by filling in the gaps between the barbs and barbules on the model's surface. Feather movement was not possible on W4. Therefore, the resulting Plasti Dip wing model (W5) allowed the effects of the surface geometry to be isolated from the effects of feather movement. 


\subsection{Set Up}

The test setup shown in Figure 29 was developed to facilitate the collection of the aerodynamic force data required to complete the objectives of this study. This system made use of a Sherline Products Motion Controller, a form of computer numerical control (CNC) rotary indexer. This device was used to adjust the angle of attack of the wing models during testing. An AeroLab six-component internal strain gage force and moment balance was used to sense forces applied to the wing during testing.
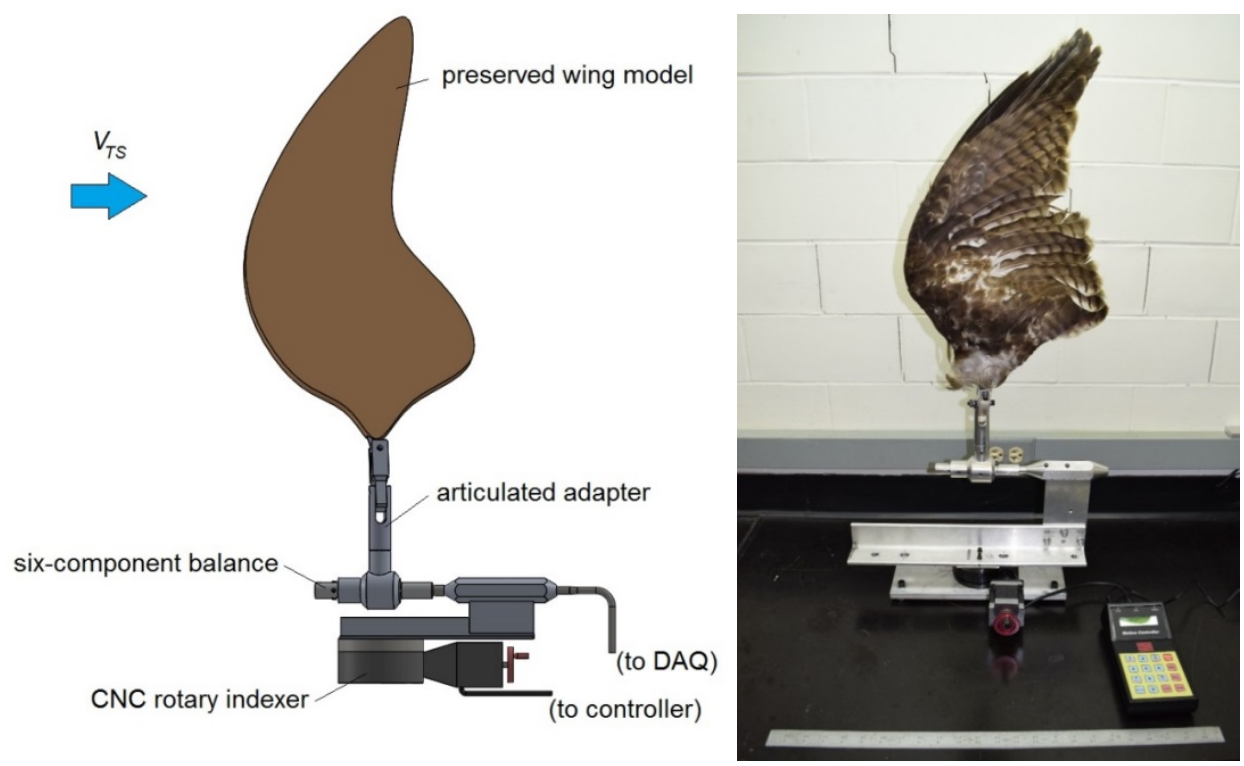

Figure 29: A Schematic Representation of the Experimental Test Setup (left) and the Actual Setup Used for Testing (right)

A National Instruments data acquisition unit (DAQ) was used to collect the aerodynamic force data throughout testing. The wing model was mounted to the six component balance using an articulated adapter. This adapter allowed the orientation of the wing to be adjusted. 
The complete setup was installed using a modular support system externally attached to the floor to the WVU closed-loop wind tunnel. Aerodynamic force data provided through the DAQ was recoded using LABVIEW software installed on a standard laptop. An example of this setup during testing can be seen in Figure 30.

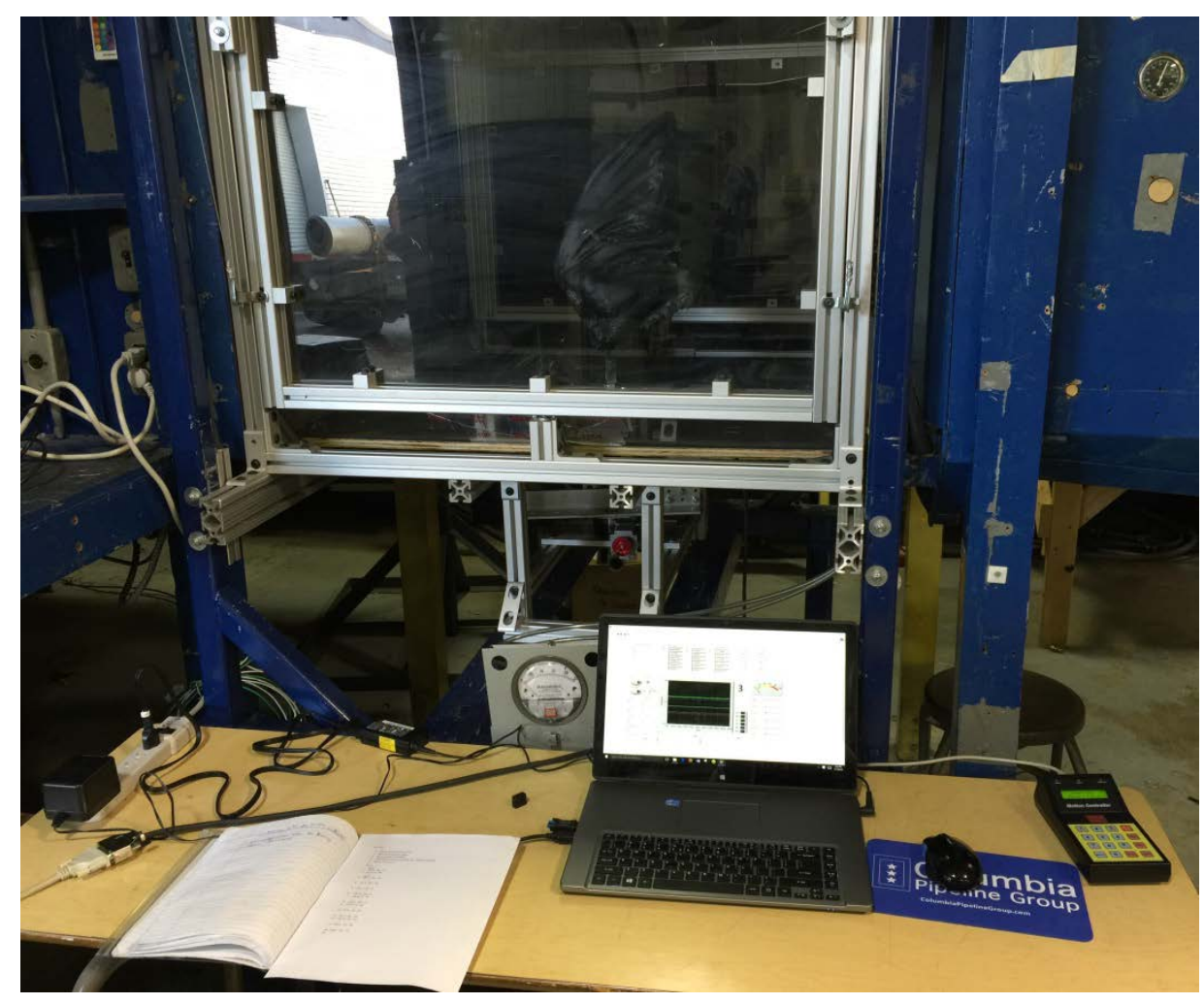

Figure 30: The Experimental Set Up as Seen During Operation 


\subsection{Equipment}

\subsubsection{WVU Low Speed Closed-Loop Wind Tunnel}

The WVU low speed closed-loop wind tunnel offered a 45” W x 32” H test section. The wind tunnel is capable of producing speeds up to 250 feet per second with a turbulence intensity (TI) of $0.2 \%$. However, for this study a maximum speed of only 34.12 feet per second was required [22]. An image of the test section can be seen in Figure 31.

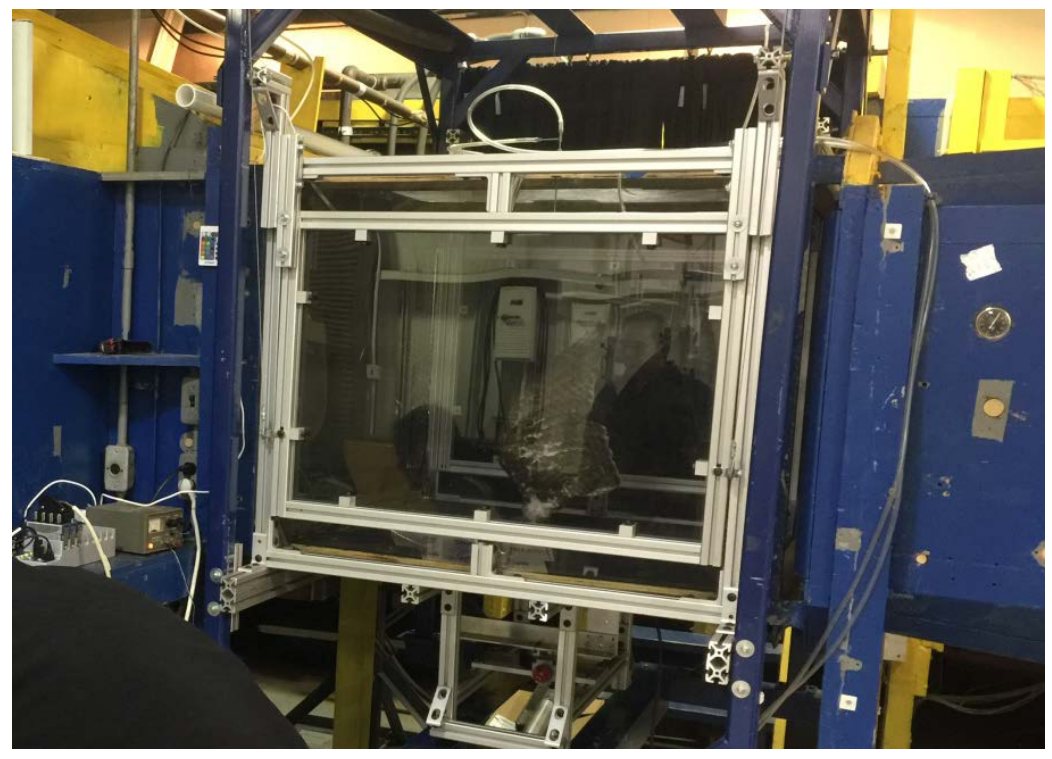

Figure 31: An External View of the WVU Closed-Loop Wind Tunnel 


\subsubsection{AeroLab Six-Component Internal Strain Gage Force and Moment Balance}

An AeroLab Six-Component Internal Strain Gage Force / Moment Balance was used to measure aerodynamic force and moment data during wind tunnel tests. This balance was mounted between the wing model being tested and the articulated adapter. The balance shown in Figure 32 was specifically manufactured by AEROLAB LLC to meet test requirements of WVU.

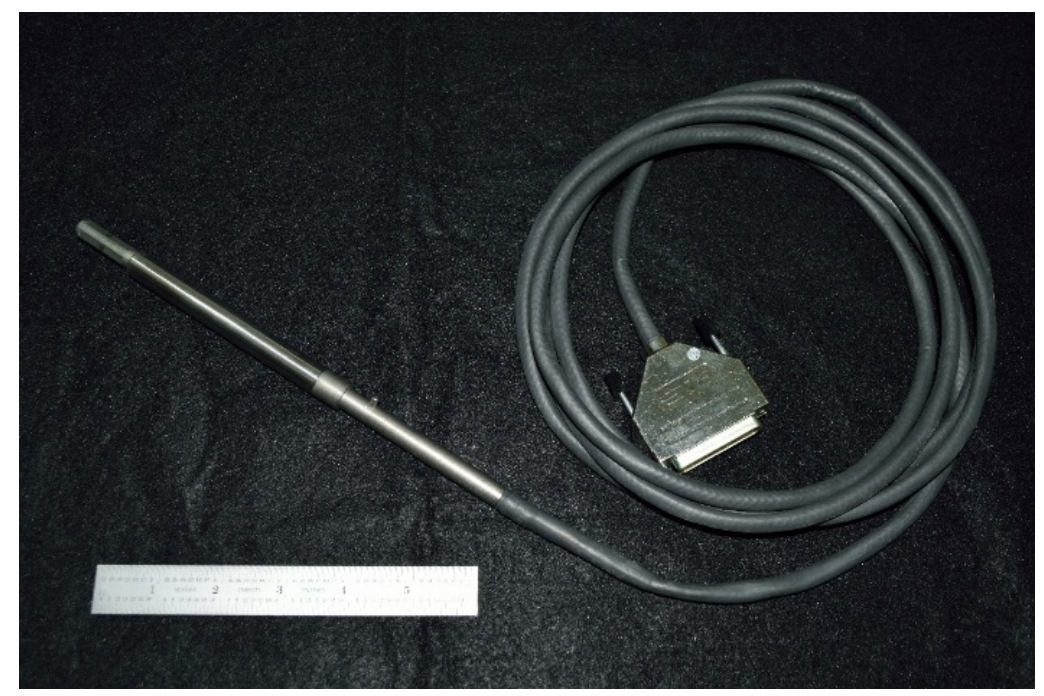

Figure 32: The AeroLab Six-Component Internal Balance Used for Testing 


\subsubsection{Data Acquisition Unit}

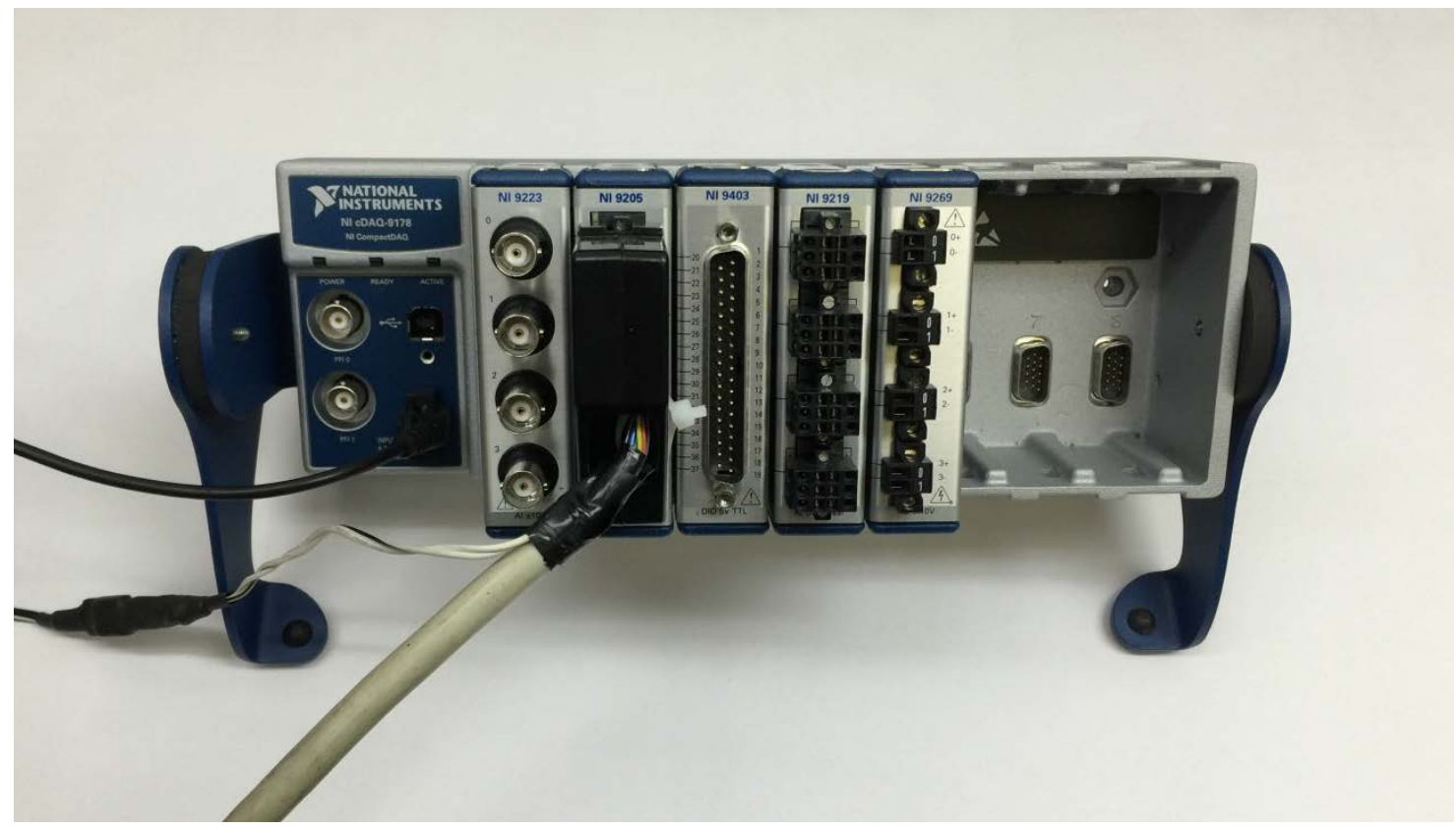

Figure 33: Nation Instruments Data Acquisition Unit

Data from the six-component balance was collected using a 14 bit National Instruments NI xDAQ-9174 data acquisition unit. During testing, this device was connected to the sixcomponent balance using a modified NI 9923 37-pin terminal block. This set up allowed the force and moments measurements of the six-component balance to be continuously streamed to a laptop computer. The laptop computer was then able to interpret and record these measurements through LabVIEW System Design Software. 


\subsubsection{Articulated Adaptor}

An image of the articulated adapter used to mount the wing models to the six component balance is shown in Figure 34. This adaptor allowed the sweep, dihedral, and incident angles of the wing to be adjusted. Through the adjustment of these angles the orientation of the wing was set to resemble the natural cruise position of the wing.

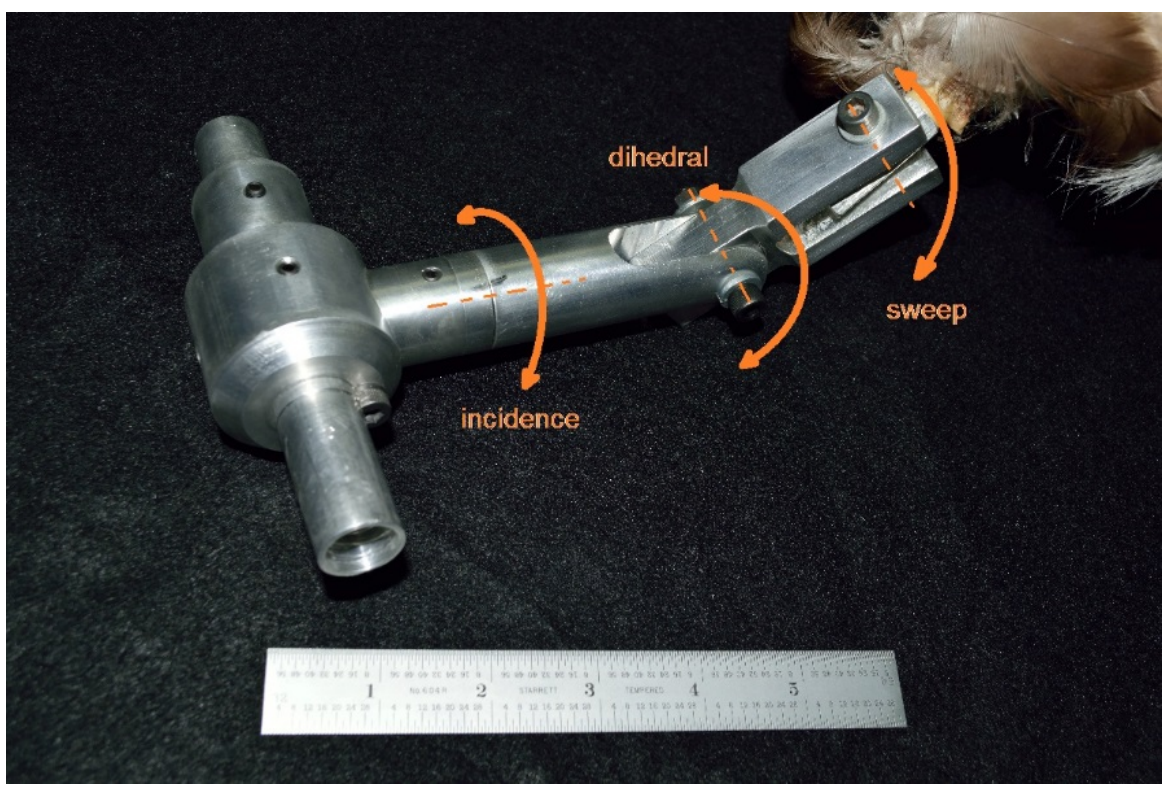

Figure 34: The Articulated Adaptor Used to Mount the Wing Models to the SixComponent Balance 


\subsubsection{Sherline Products 4" Rotary Table}

The Sherline Products 4" Rotary Table shown in Figure 35 was used in the experimental set up to provide an easy and accurate way to quickly adjust the angle of attack of wing models. This device allowed the angle of attack of the wing models to be electronically adjusted in increments as small as six thousandths of a degree. The rotary table also incorporated a locking mechanism to prevent the positon of the table from drifting during operation [23].

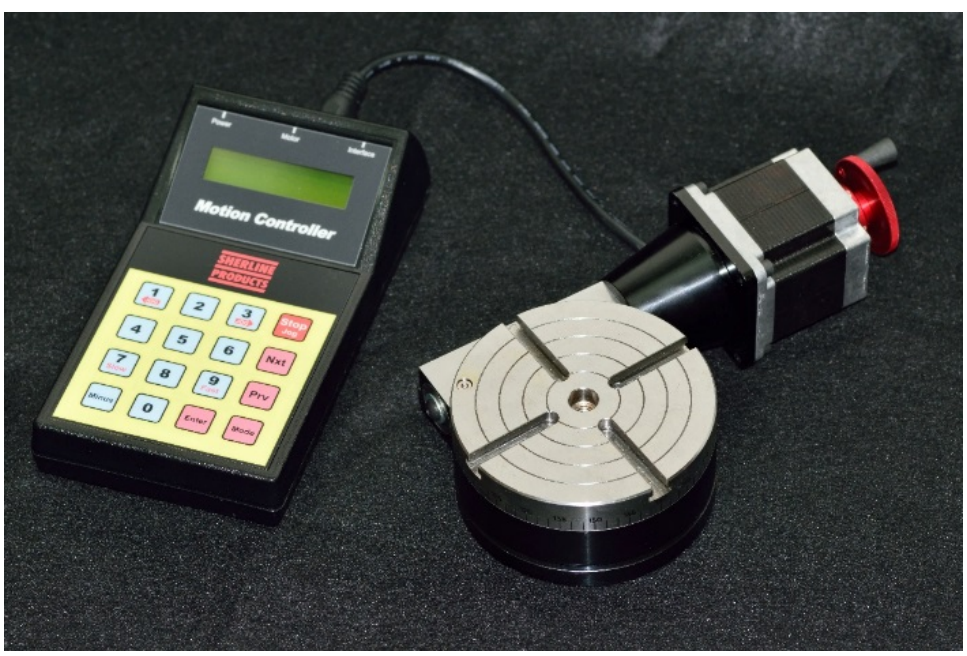

Figure 35: An Image of the Sherline Products Rotary Indexer Used in the Experimental Set Up 


\subsubsection{DAVID SLS-2 Structured Light 3D Scanner}

In order to evaluate the macroscopic differences in shape between the natural and cast wings, three dimensional (3D) scans of W3 and W4 were taken using a DAVID SLS-2 Structured Light 3D Scanner shown in Figure 36. This device allowed each model to be scanned with a resolution of up to $0.06 \mathrm{~mm}$. The resulting scans were then exported to Windows 3D Builder as Stereo Lithography or STL files. STL is a format commonly used for rapid prototyping and 3D printing. This allowed the 3D models to be generated from the scans.

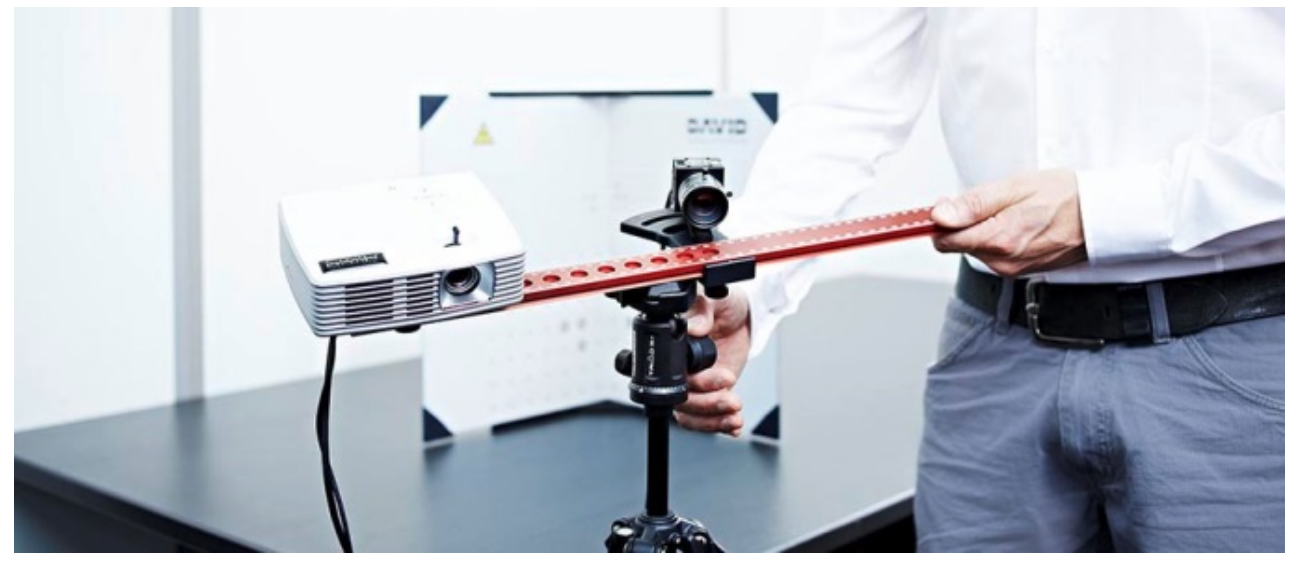

Figure 36: A DAVID SLS-2 3D Scanner Assembled for Use [24] 


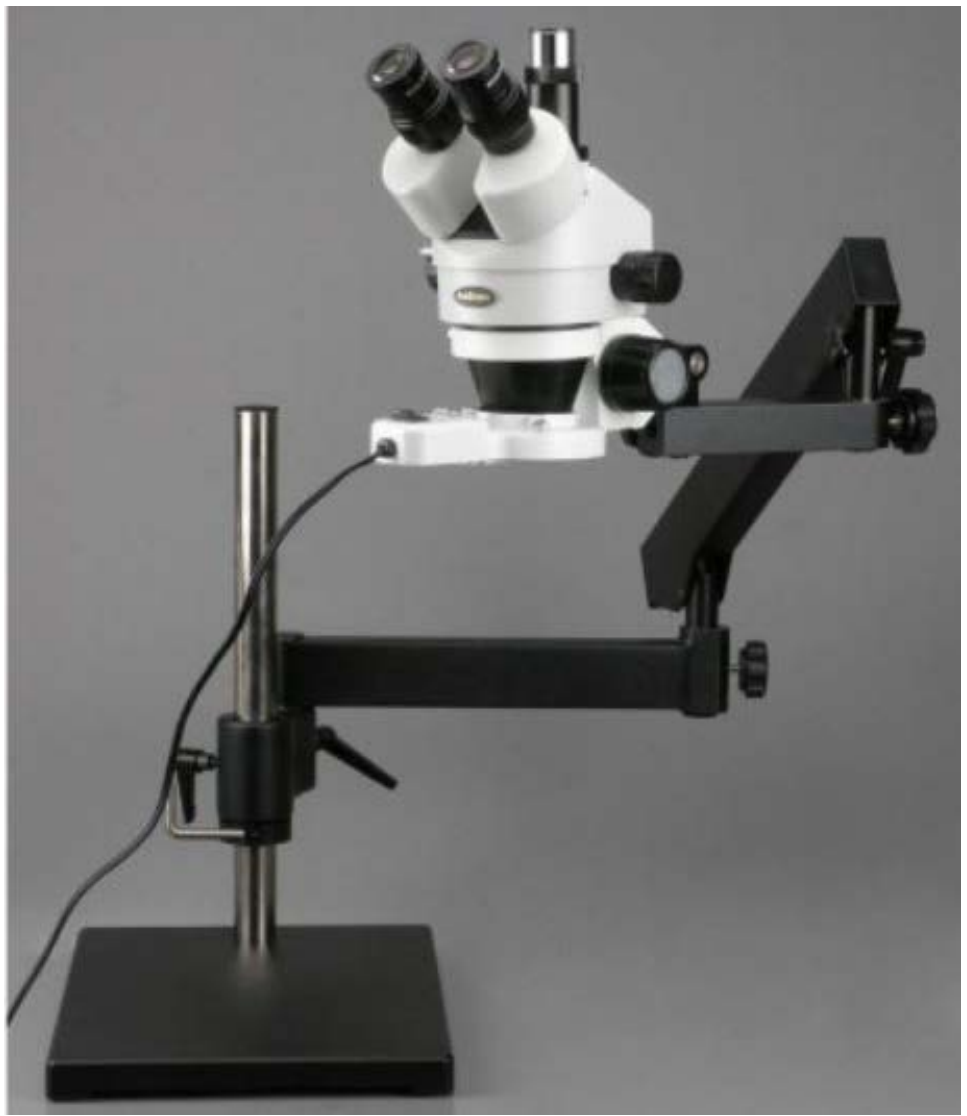

Figure 37: The AmScope SM-7 Microscope Used to Document the Surface Geometry of the Wing Models [25]

Photographic documentation of the surface geometry of each wing model was collected using an AmScope SM-7 trinocular zoom microscope shown in Figure 37. This microscope allowed pictures of the wing surface to be taken between $7 \mathrm{x}$ and $180 \mathrm{x}$ magnification. Surface depth measurements were also recorded by measuring the change in microscope's focal length between the highest and lowest spots on the wing. 


\section{Chapter 4: Method}

\subsection{Calculation the Mean Chord Length, Area, and Aspect Ratio of the Wing Models}

The area and arithmetic mean chord length of the models was found using a simple piece wise approach. It is recognize that the mean aerodynamic chord is normally used for similar studies. However, due to the complex shape of the wing, no closed form solution could be found. Traces of W1 and W4, shown in Figure 38, were made on large sheets of paper. The chord length of the wing was measured at one inch intervals along the span of the each wing trace.

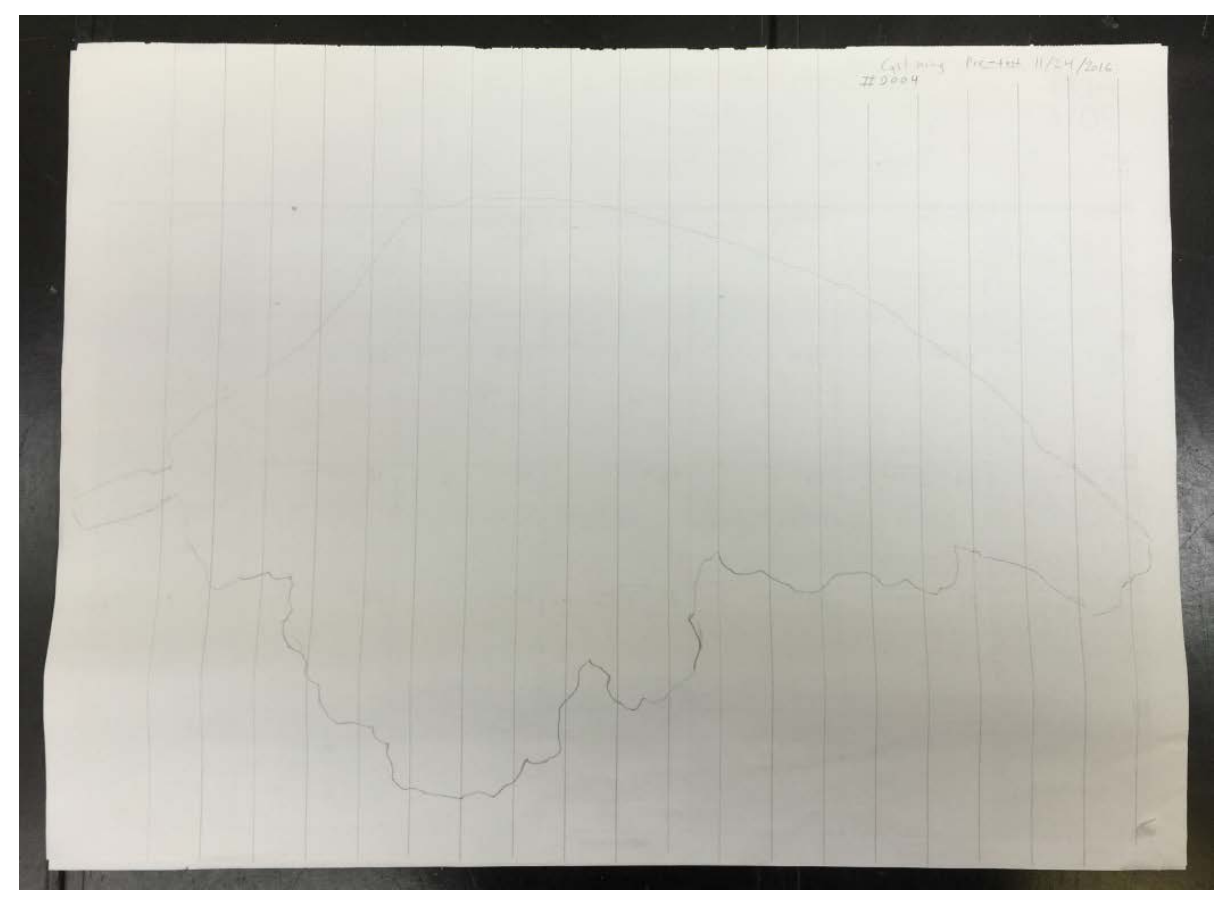

Figure 38: The Trace of the Cast Wing Model Used to Find the Area and Mean Chord Length 
From the recorded chord lengths, the mean chord length of each of the two models was found. Since the chord length was taken at one inch intervals, the area of the each wing was the sum of the areas of the rectangles separated by the location of each chord length measurement.

Through this method, the mean chord length was found to be 6.993 in or $0.583 \mathrm{ft}$. The wing area (A) was then found to be $133.056 \mathrm{in}^{2}$ or $0.924 \mathrm{ft}^{2}$. When measured from the base to the wing tip, the span $(B)$ of the wing was 18.5 in or $1.542 \mathrm{ft}$. Once these values were obtained, the aspect ratio (AR) of 2.573 was calculated using the following equation.

$$
A R=\frac{B^{2}}{A}
$$

\subsection{Alignment of Wing Models}

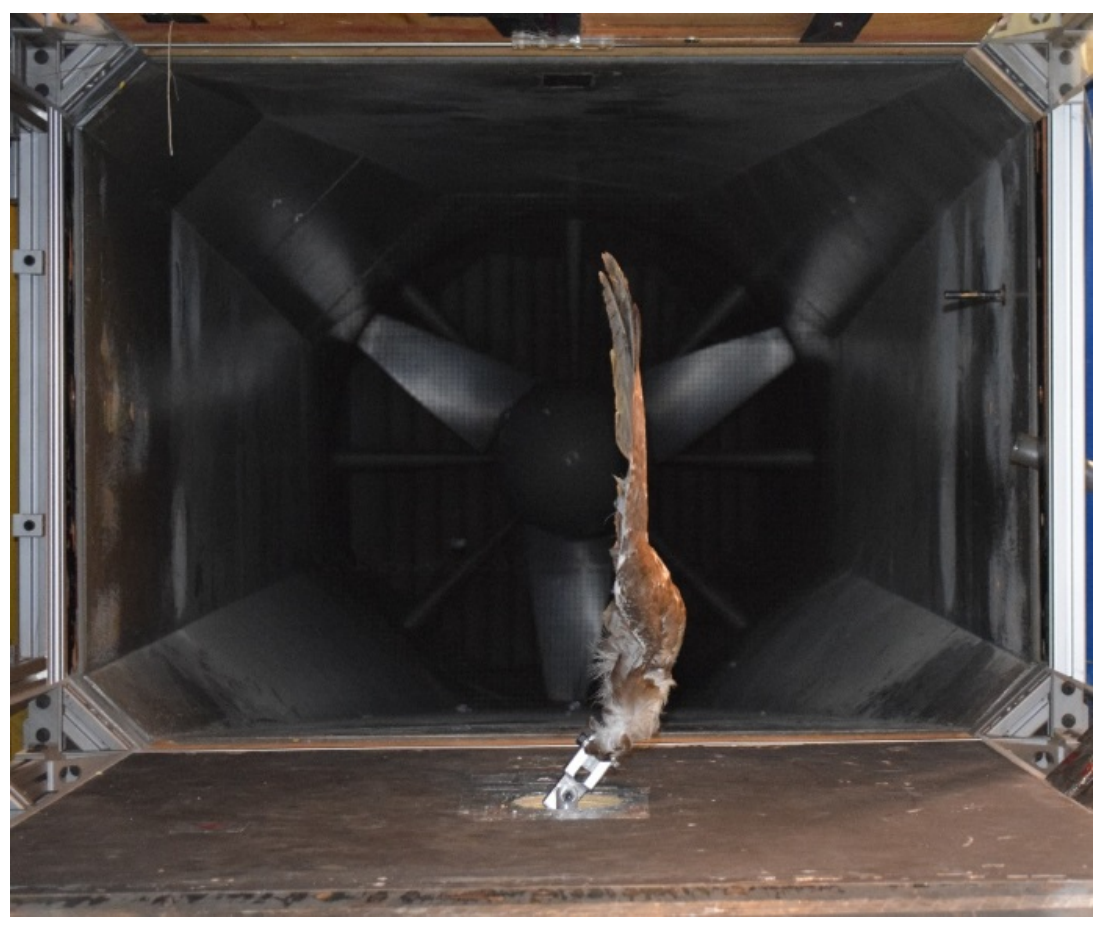

Figure 39: The Preserved Wing Model Aligned and Prepared for Testing 
Before tests to collect aerodynamic force data could be conducted, the wing model being tested had to be aligned properly. Using the articulated adapter the sweep and dihedral angles of the wing were set to resemble a wing in the gliding phase. The incident angle was set to best exhibit zero degrees angle of attack. The wing model was only removed from the articulated adapter after tests were conducted on W3. Therefore, these angles were only required to be set for W1 and W4.

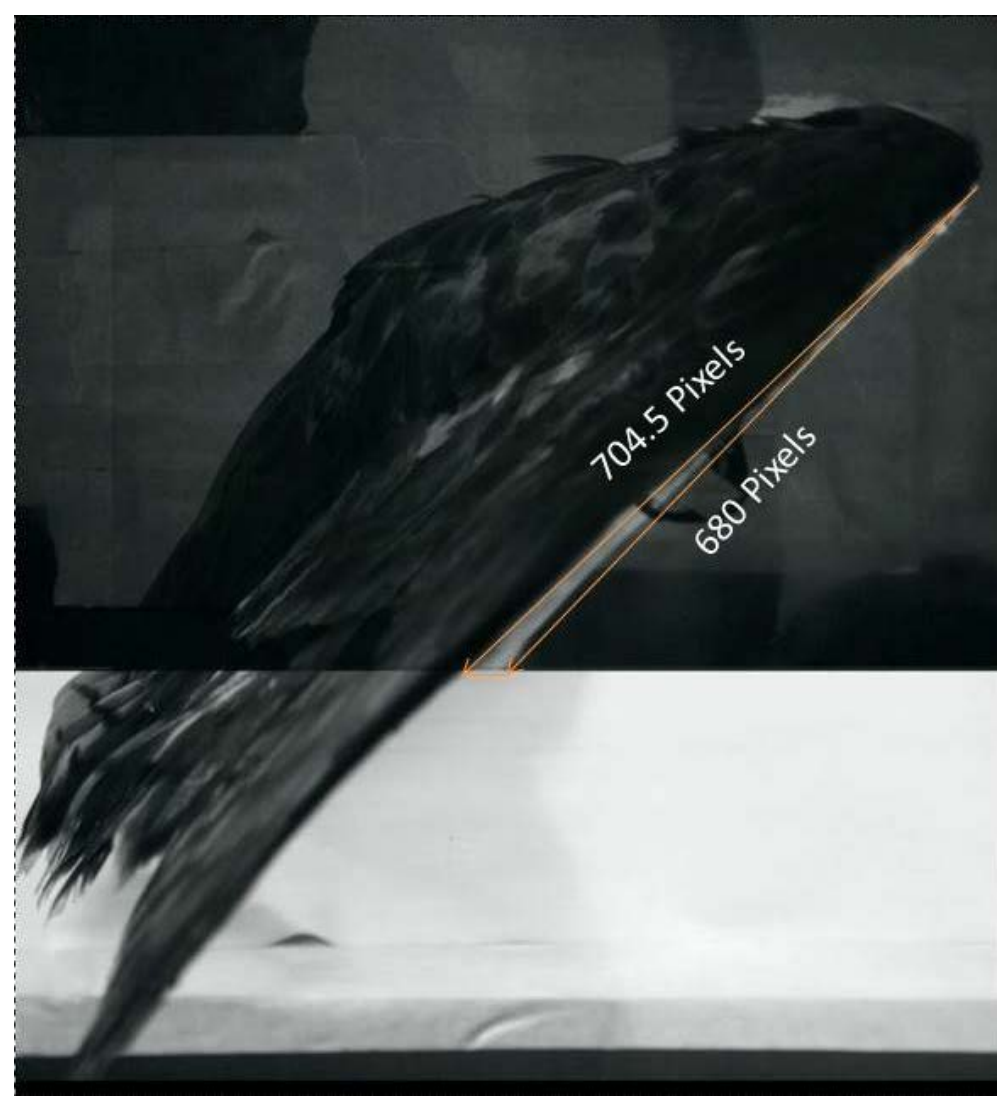

Figure 40: A Comparison of Wing Model Pictures used for Alignment

In order to reorient W4 with the alignment of W1, photographic documentation was used to compare the orientation of two wings. Adjustments to the sweep and dihedral angles of W4 were made using the articulated adaptor. However, the incident angle was compensated for 
during testing by offsetting the angle of attack by $15.15^{\circ}$ in the negative or clockwise direction as seen in Figure 40 . The $15.15^{\circ}$ offset was found using the distance measured between two common points on each wing in the following calculation.

$$
\cos ^{-1}\left(\frac{680}{704.5}\right)=15.15^{\circ}
$$

\subsection{Collection of Aerodynamic Force Data}

Aerodynamic force data was collected using the described experimental set up and equipment. For each wing model the angle of attack was changed in increments of primarily

four degrees. Aerodynamic force data was recorded at each of the determined increment as the angle of attack ranged between $-40^{\circ}$ to $40^{\circ}$. This procedure was repeated for several runs at 10 test section head pressures between .065 and .205 inches of water. Based on the mean chord lengths of the models and local atmospheric conditions, $6.13 \times 10^{4}$ to $1.09 \times 10^{\wedge} 4$ was the resultant Reynolds number test range. 


\subsection{Comparison of the Macroscopic Shape of the Cast and Biologic Wing Models}

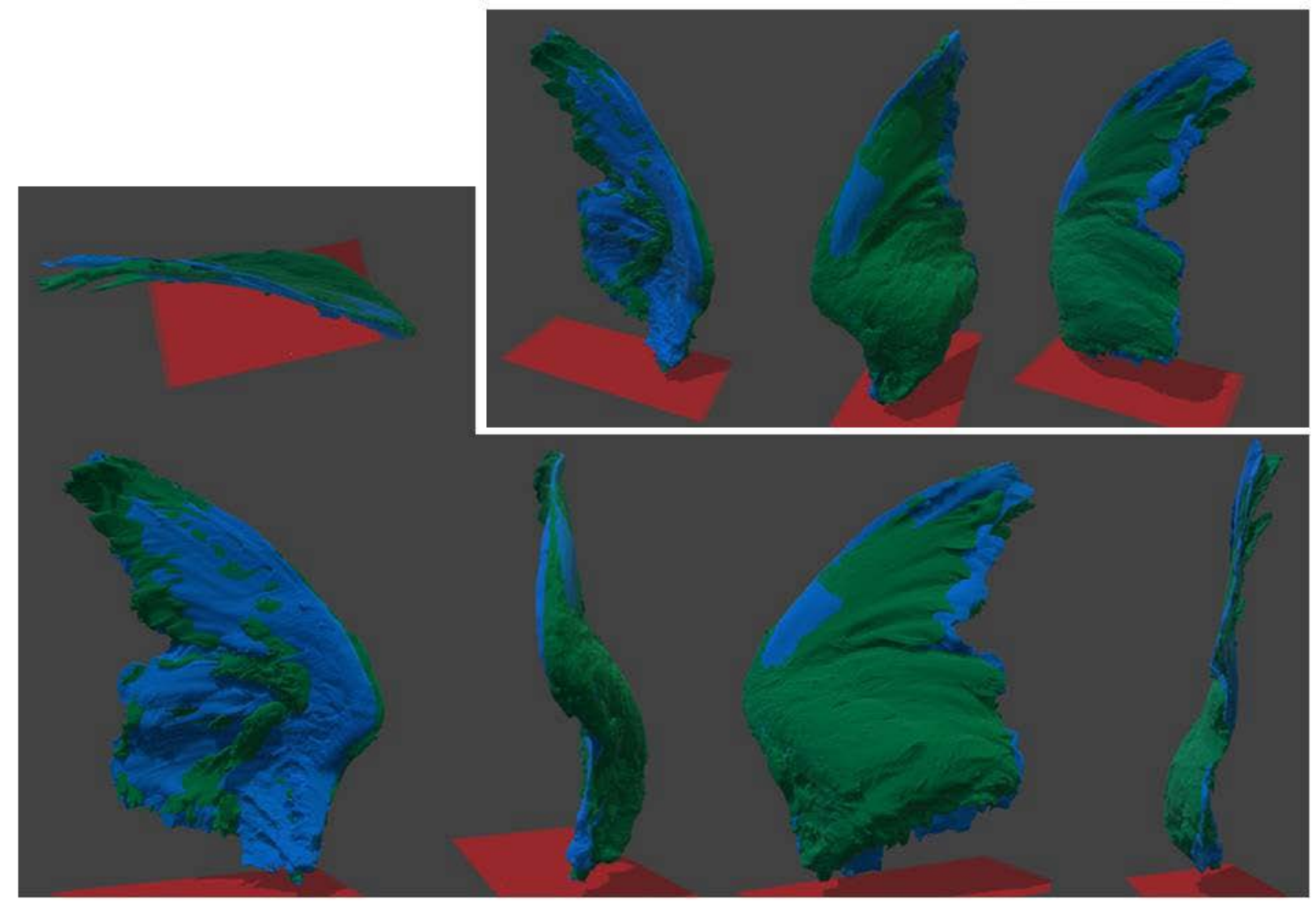

Figure 41: Merged 3D Scans of the Cast (blue) and Polyurethane Coated (green) Wing Models

3D scans taken of W3 and W4 were used to compare the macroscopic shapes of the wings. Using Windows 3D Builder software, the 3D scans of the wings were converted to a solid 3D model. The color blue was assigned to the scan of W4. Green was assigned to the scan of W3. The volumes of the two model scans were compared. W3 had a volume of $63 \mathrm{in}^{3}$. The volume of W4 was $41.26 \mathrm{in}^{3}$. As a result, the natural wing was found to have a $34.6 \%$ larger volume. However, there was a much smaller difference in the area of the two wings. The area of W3 was $313.08 \mathrm{in}^{2}$. W4 had an area of $281.26 \mathrm{in}^{2}$. As a result W3 only a $10.1 \%$ larger area. 
The 3D models were then merged together and aligned to form the final model as seen in Figure 41. From this final model, differences between the macroscopic shapes of W3 and W4 could easily be seen and evaluated qualitatively. By examining the final model, it was determined that the macroscopic shape of the wings was sufficiently similar to support the completion of the primary objectives.

\subsection{Evaluation of Surface Geometry Differences Across the Wing Models}
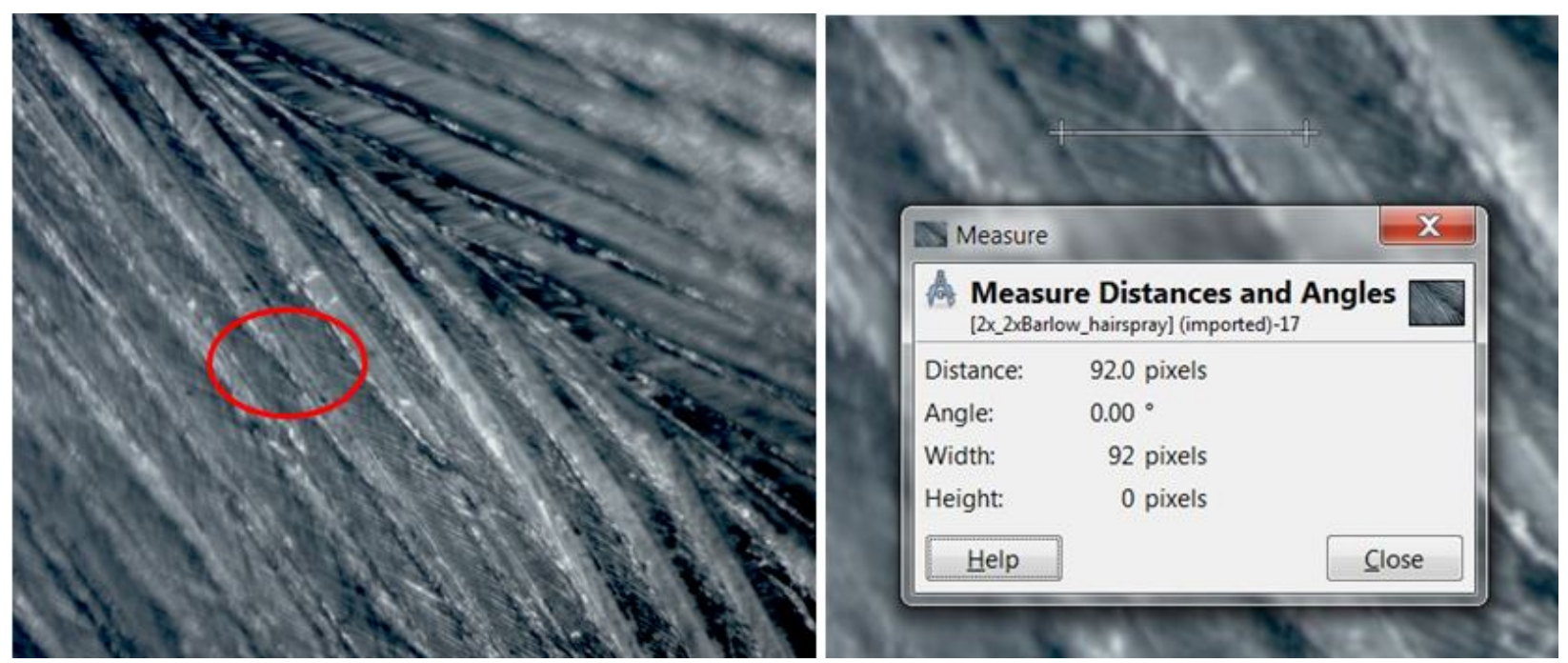

Figure 42: An example of the Distance between Barbs Measured on the Preserved Wing Model using Image Manipulation Software

Alterations in the surface geometry between the wings were evaluated using pictures taken with the AmScope SM-7 microscope at 14, 40, and 80 times magnification. Although the microscope was capable of a maximum magnification of 90x, the quality of the image at the maximum magnification were too poor to be used. Therefore, pictures taken at this magnification were not used. 


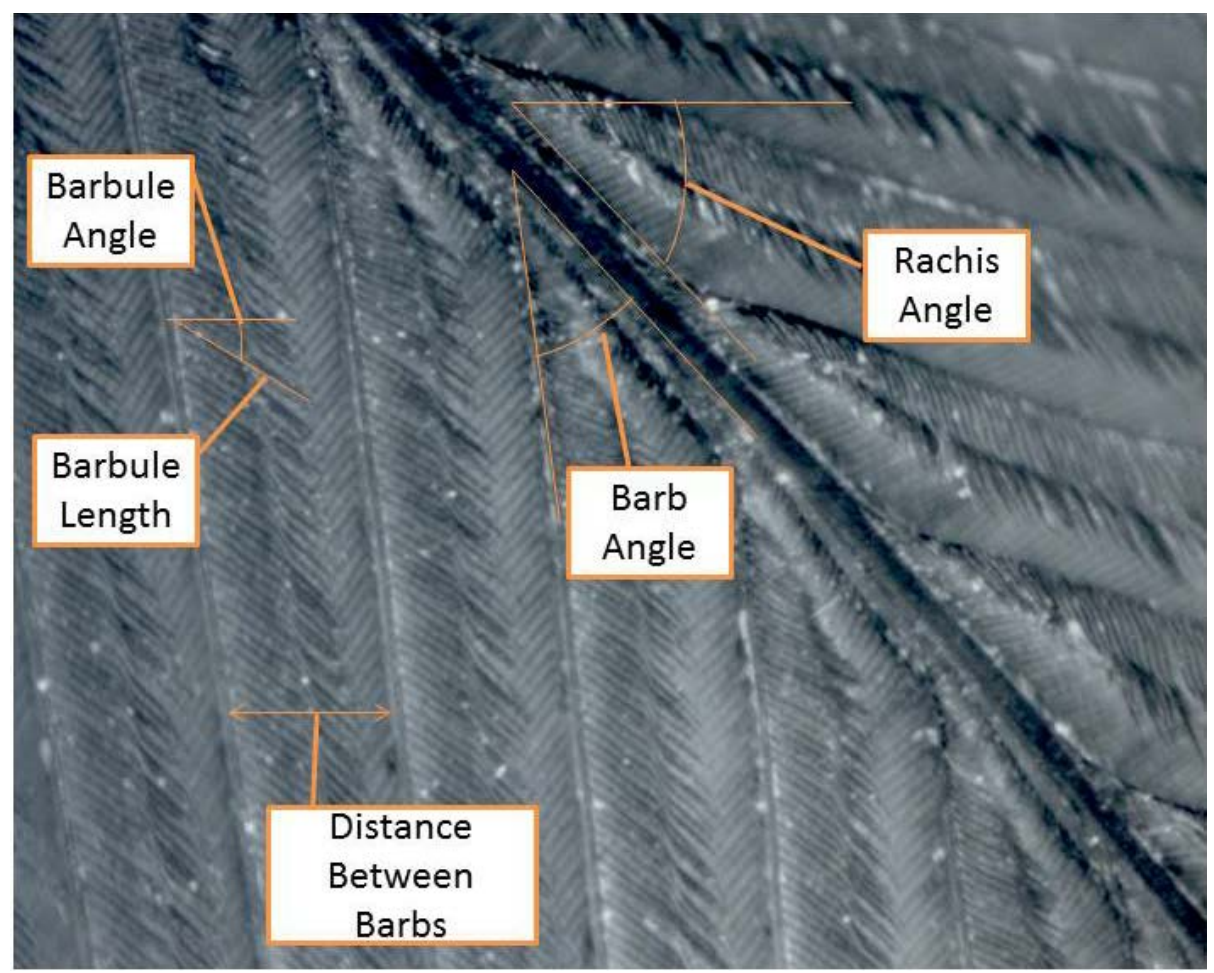

Figure 43: Examples of Surface Geometry Measurements

Figure 43 provides an example of the measurements taken from the microscope images. Gimp, a common image manipulation program, was used to measure the distances and angles. Measurements made using Gimp were given in pixels. Therefore, a conversion factor found through calibration of the software, was used to convert the measurements from pixels to microns. The rachis angle ( $\varsigma)$, barb angle $(\beta)$, barb to barb distance $(\zeta)$, barbule length $(\Gamma)$, and barbule to barbule distance $(\lambda)$ were all measured using this technique.

\subsection{Finding the Feather Frequency and Amplitude of Wing Models}

The frequency and amplitude of four types of feathers from $\mathrm{W} 1, \mathrm{~W} 2$, and $\mathrm{W} 3$, were found from high speed videos taken of each model. The four types of feathers studied were the 
primaries, secondaries, coverts, and secondary coverts. For each wing these videos were taken at $-40^{\circ}, 0^{\circ}$, and $40^{\circ}$ angle of attack. A head pressure of 0.125 inches of water was applied to each wing while the high speed videos were taken.

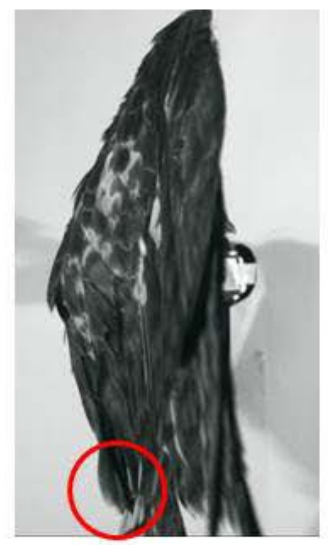

Image 00028

$\mathrm{T}=.28 \mathrm{sec}$

Max Amplitude

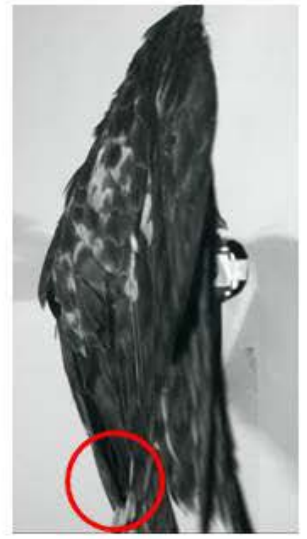

Image 00043

$\mathrm{T}=.43 \mathrm{sec}$

Min Amplitude

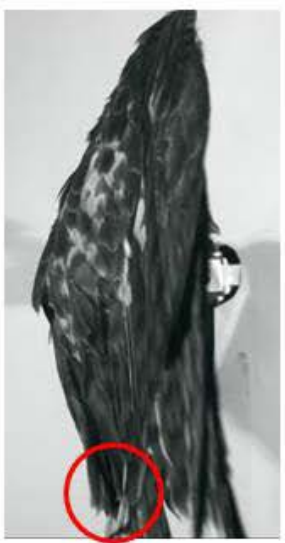

Image 00059 $\mathrm{T}=.59 \mathrm{sec}$ Max Amplitude

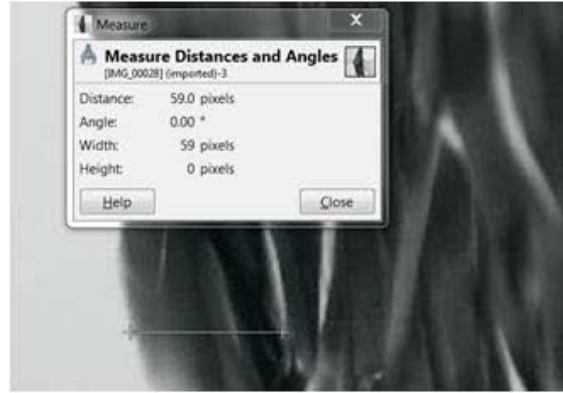

Image 00028 Measurement $59 x=.400^{\prime \prime}$

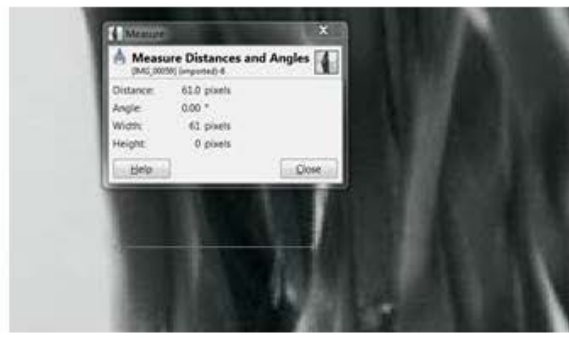

Image 00059 Measurement

$61 x=.413^{\prime \prime}$

Figure 44: An Example of Feather Amplitude and Frequency Measurements Taken from the Preserved Wing Model

Using Adapter, a common video, image, and audio converter, the high speed videos were converted into as many as 100 high resolution images per second of video. From the resulting images, the time at which the maximum and minimum deflection of a feather occurred could be found. Maximum and minimum displacement of the selected feathers was measured using Gimp software.

Once the maximum and minimum displacement and the time between the two images were known, the amplitude and frequency of that feather could be found. Feather displacement 
measurements were originally found in pixels. The distance in pixels was then converted to inches using a conversion factor found by measuring a known distance on the wing. An example of the measurements used for this process can be seen in Figure 44. When possible, this process was completed using the same feathers and reference points on each wing. The following equations were then used to find the frequency and amplitude of the feathers.

$$
\begin{gathered}
\text { Period }=\text { Time }_{\text {Max amp }}-\text { Time }_{\text {Min amp }} \\
\text { Frequency }=\frac{1}{\text { Period }} \\
\text { Amplitude }=\frac{\text { Avg. Max Displacement }}{2}
\end{gathered}
$$




\section{Chapter 5: Results}

\subsection{Feather Motion Reduction between Wing Models}

The following figures show that the amplitude of the feather motion on W2 and W3 was reduced with the application of hair spray or polyurethane. In many cases as much as $100 \%$ was found. One example of this was the covert feathers as seen in Figure 45.
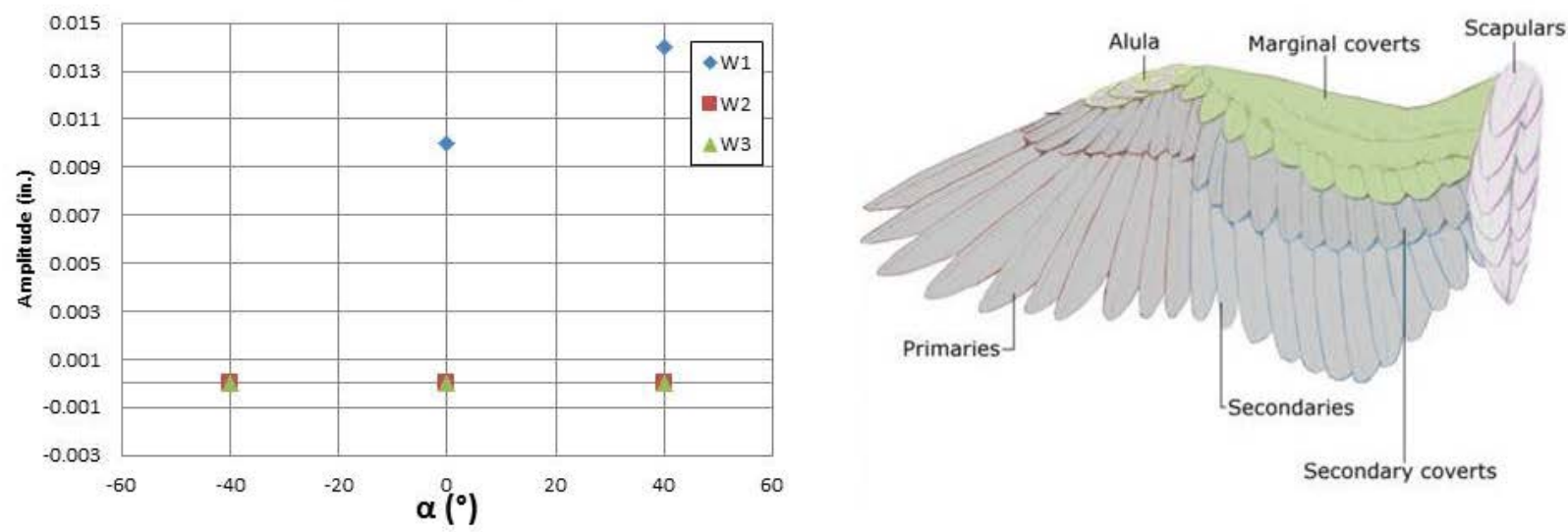

Figure 45: Changes in Covert Feather Amplitude

Figure 46 shows that secondary covert feather motion was also drastically affected by either application. However, the secondary coverts of W2 were not completely inhibited at $\alpha=$ $40^{\circ}$ and $\alpha=0^{\circ}$. Although, the amplitude of these feathers on W2 was greatly reduced, some feather motion still occurred. 

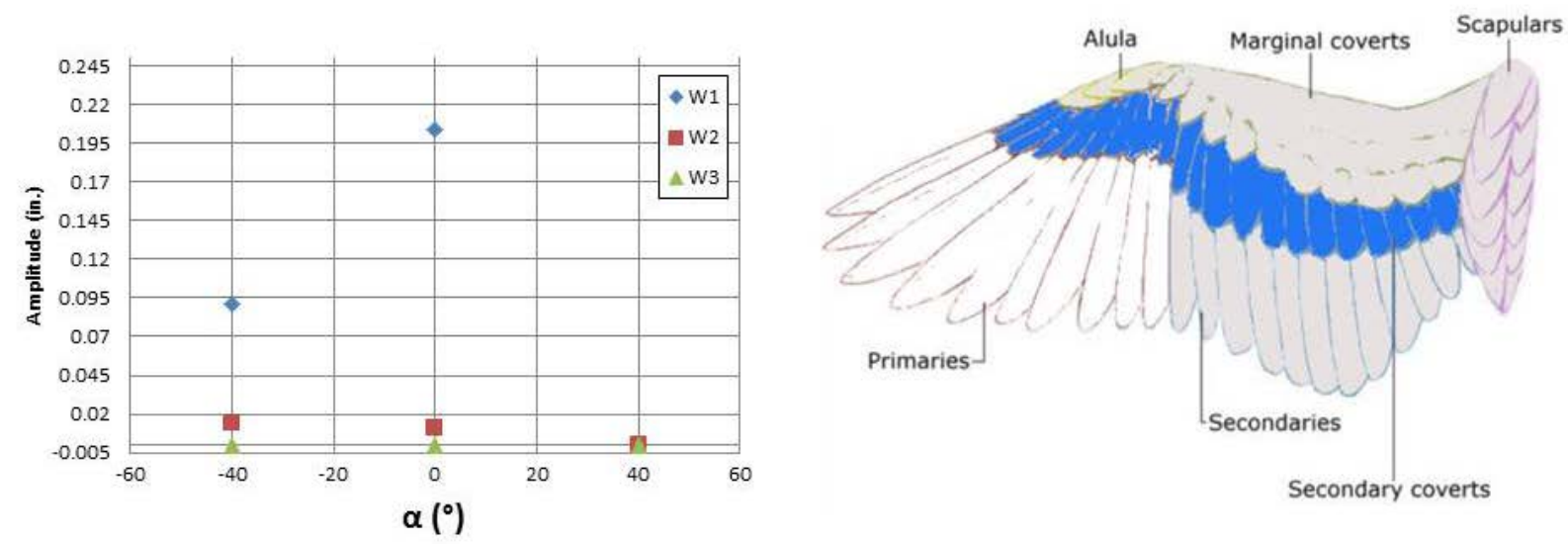

Figure 46: Changes in Secondary Covert Feather Motion

The amplitude of the primary feathers was less affected by these applicants as seen in Figure 47. When hair spray was applied, feather amplitude was noticeably decreased when the angle of attack was below $0^{\circ}$. The amplitude of the hair sprayed primaries then increased at $\alpha=$ $40^{\circ}$. The primary feathers of W3 reacted much differently. Feather amplitude was drastically decreased at $\alpha=40^{\circ}$, but the same amplitude as the primaries of W1 occurred at $\alpha=0^{\circ}$.
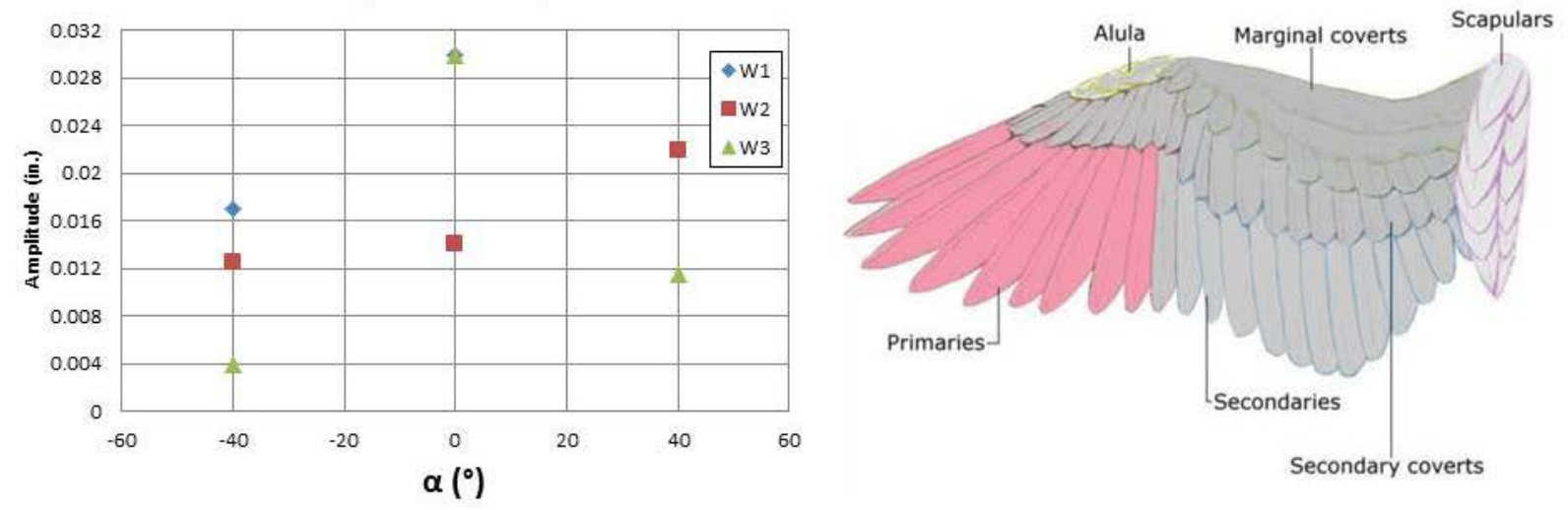

Figure 47: Changes in Primary Feather Amplitude 
Figure 48 shows that secondary feather amplitude was also decreased when hair spray or polyurethane were applied. The changes in secondary feather amplitude were similar that of the primaries in Figure 47. However, the amplitude of the primaries at $\alpha=40^{\circ}$ was higher than the W1.
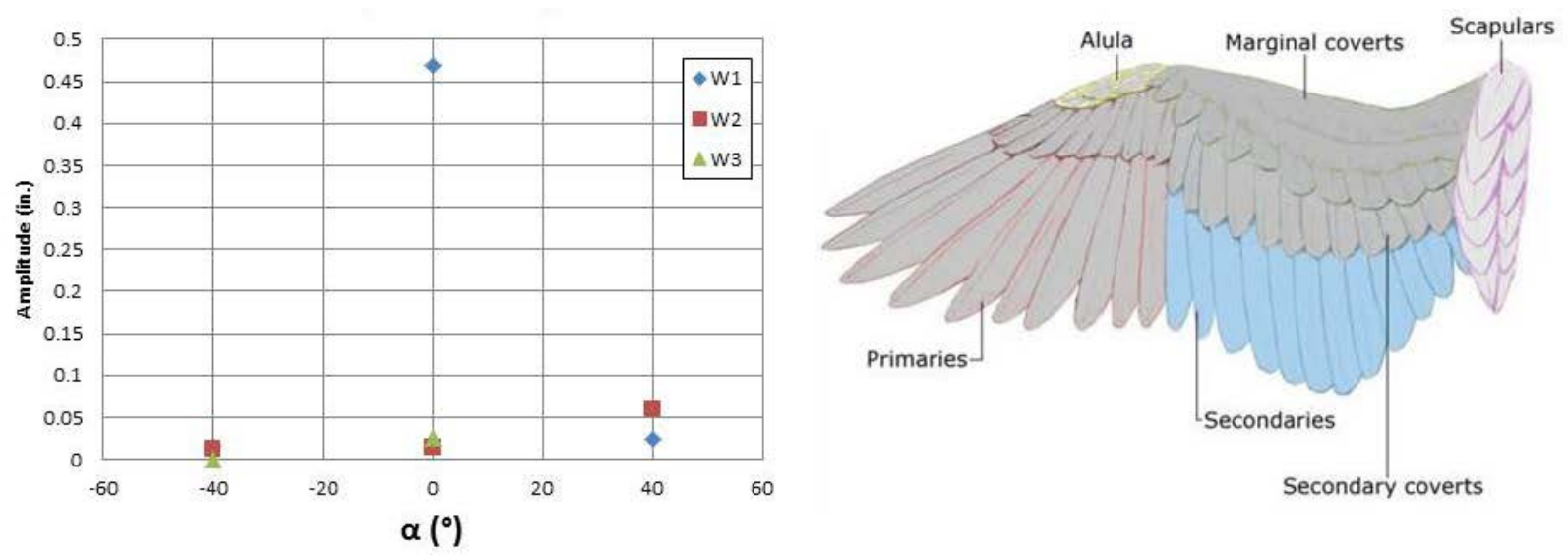

Figure 48: Changes in Secondary Feather Amplitude

The covert feather frequencies found in this study greatly differ from those observed on a trained American Kestrel in flight at WVU. Though a similar video analysis, researchers at WVU found that during the Kestrel's filmed flights, the covert feather frequency ranged from 14 $\mathrm{Hz}$ to $330 \mathrm{~Hz}$ [26]. The maximum covert feather frequency found in this study was $3 \mathrm{~Hz}$. The true cause in the frequency differences between this study and the American Kestrel observations at WVU may be the attributed to the static test conditions used for this study as compared to the dynamic conditions experienced by a live bird in flight [26].

The application of hair spray and polyurethane cause the frequencies of most feather groups on the test wings to reduce. Hairspray increased the frequency of the covert feathers at $\alpha$ 
$=40^{\circ}$ when compared to W1. Figure 49 shows that both surface applications inhibited covert feather motion when $\alpha$ was $-40^{\circ}$ or $0^{\circ}$.
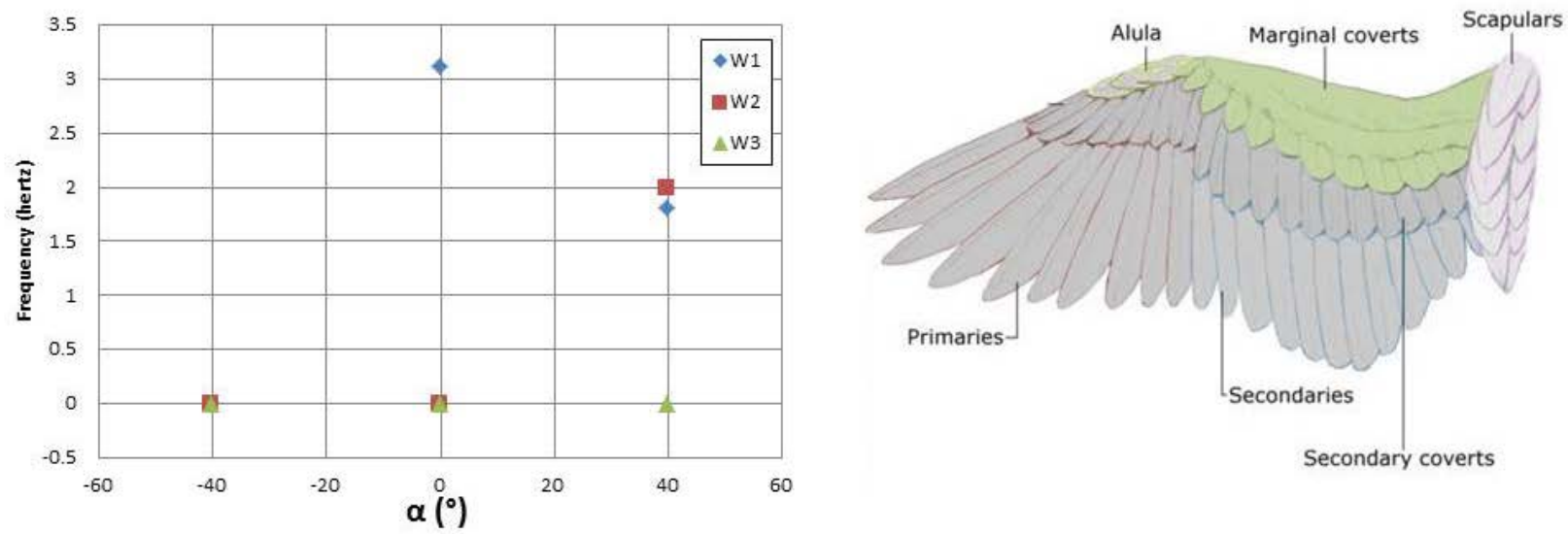

Figure 49: Effects of surface applications on Covert Feather Frequency

Secondary covert feather frequency was higher for W2 than for W1 or W3 at both $\alpha=-$ $40^{\circ}$ and $\alpha=0^{\circ}$ as seen in Figure 50. However, the application of polyurethane completely inhibited these feathers at every $\alpha$ test. At $\alpha=40^{\circ}$, secondary covert feather motion was inhibited for all three wings.
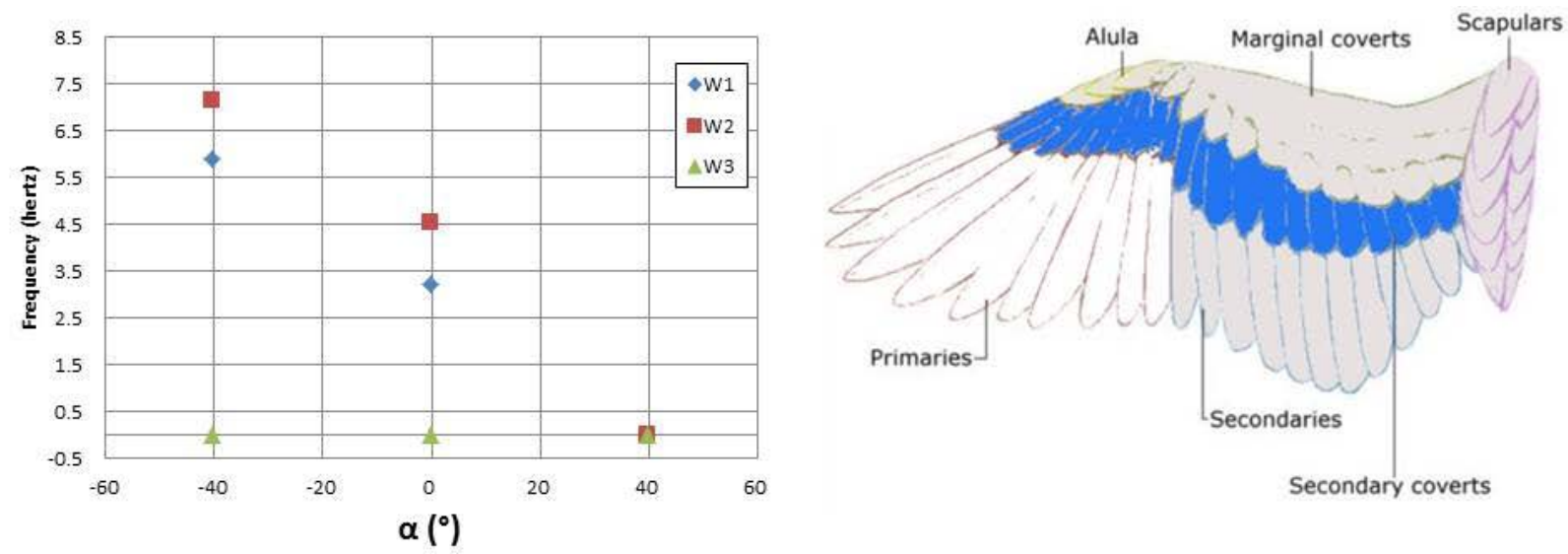

Figure 50: Effects of Surface Applications on Secondary Covert Feather Frequency 
The application of these hair spray and polyurethane had a more consistent effect on the frequency of the primary. Figure 51 shows that hair spray reduced the frequency of the primaries on W2. Polyurethane then further reduced the frequency of the primary feathers on W3.
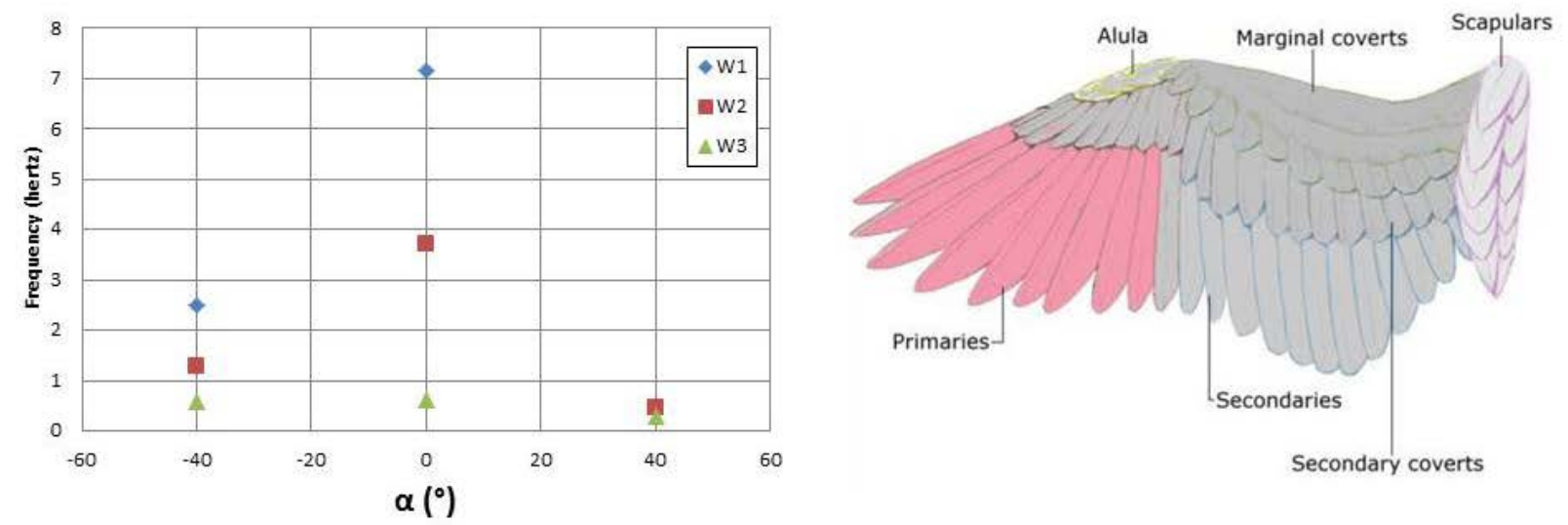

Figure 51: Effects of Surface Applications on Primary Feather Frequency

Hair spray and polyurethane had an effect on the secondary feathers similar to that of the primary feathers. Figure 52 shows that the application of hair spray caused W2 to have lower secondary feather frequencies than W1. Applying polyurethane then caused the frequency of the secondaries on W3 to be the lowest. 

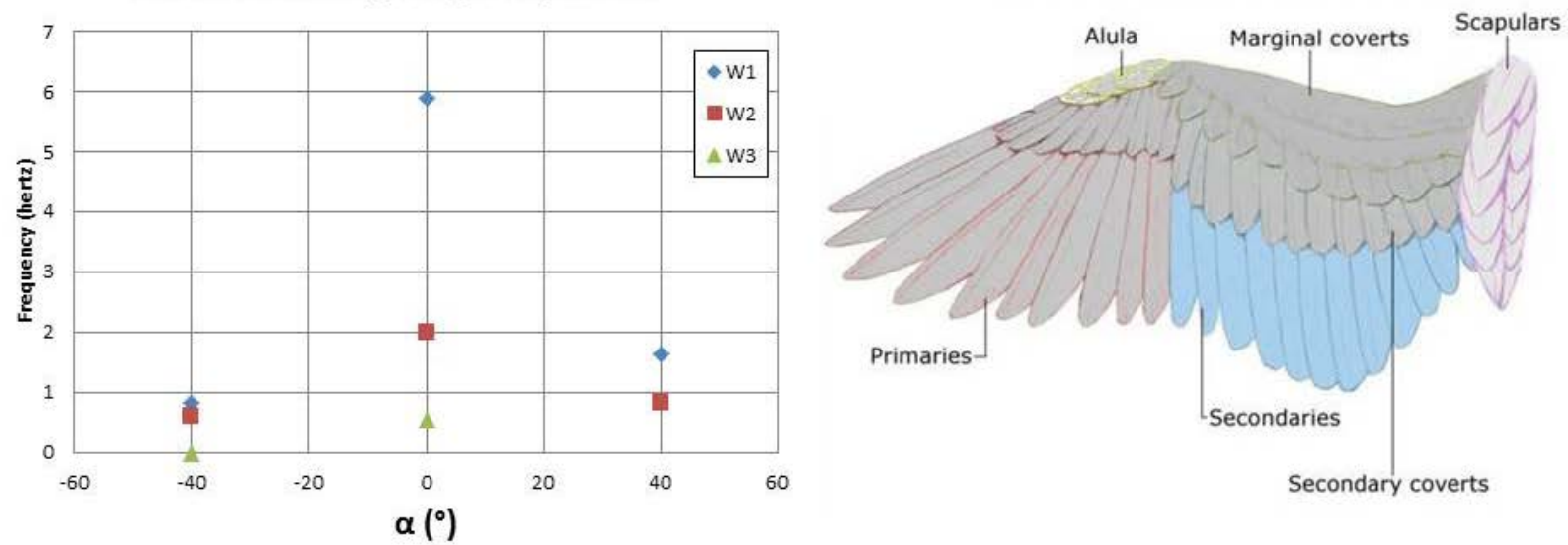

Figure 52: Effects of Surface Applications on Secondary Feather Frequency

A qualitative representation of the change in feather motion between W1 and W2 is provided in Table 3 . At $\alpha=40^{\circ}$ the view of the primaries was blocked by part of the equipment setup. Therefore, this information could not be provided. However, the frequency and amplitude reduction of the primaries on W2 was found to be about 50\% when the primaries were in view.

Table 3: Change in Feather Motion with the Application of Hair Spray

\begin{tabular}{|c|c|c|c|c|}
\hline \multicolumn{5}{|c|}{ W1 VS. W2 } \\
\hline $\boldsymbol{\alpha}\left(^{\circ}\right)^{\circ}$ & Head (in. H2O) & Feather Type & Frequency \% Decrease & Amplitude \% Decrease \\
\hline-40 & 0.125 & Primaries & $49 \%$ & $26 \%$ \\
\hline-40 & 0.125 & Secondaries & $25 \%$ & $25 \%$ \\
\hline-40 & 0.125 & Coverts & $0 \%$ & $0 \%$ \\
\hline-40 & 0.125 & Secondary Coverts & $-21 \%$ & $90 \%$ \\
\hline 0 & 0.125 & Primaries & $48 \%$ & $53 \%$ \\
\hline 0 & 0.125 & Secondaries & $66 \%$ & $97 \%$ \\
\hline 0 & 0.125 & Coverts & $100 \%$ & $100 \%$ \\
\hline 0 & 0.125 & Secondary Coverts & $-40 \%$ & $95 \%$ \\
\hline 40 & 0.125 & Primaries & Not Available & Not Available \\
\hline 40 & 0.125 & Secondaries & $49 \%$ & $-150 \%$ \\
\hline 40 & 0.125 & Coverts & $-10 \%$ & $57 \%$ \\
\hline 40 & 0.125 & Secondary Coverts & $0 \%$ & $0 \%$ \\
\hline
\end{tabular}


Although the motion of the secondary feathers of was reduced on W2, it was less consistent. The frequency and amplitude were both reduced when $\alpha=-40^{\circ}$ by $25 \%$. However, at $\alpha=0^{\circ}$, the frequency and amplitude reduction of the secondary feathers increased to $48 \%$ and $97 \%$, respectively. When $\alpha=40^{\circ}$ the frequency reduction returned to $48 \%$, but the amplitude of these feathers on W2 increased by $150 \%$ when compared to W1.

A pattern similar to the secondary feathers was observed in the covert feathers. Covert feather motion was completely inhibited on W2 except at $\alpha=40^{\circ}$. At this angle of attack, both the frequency of the coverts increased by $10 \%$ when compared to W1, but the amplitude of these feathers attached to W2 decreased by 57\% at this angle of attack.

The application of hair spray had the opposite effect on the motion of the secondary coverts. Although the amplitude decreases in every case, the frequency of these feathers increased. It first increased by $21 \%$ at $\alpha=-40^{\circ}$, then $40 \%$ at $\alpha=0^{\circ}$. Secondary feather motion was then inhibited on both $\mathrm{W} 1$ and $\mathrm{W} 2$ at $\alpha=40^{\circ}$.

In summary, applying hair spray reduced primary and secondary feather frequency on W2. The amplitude of these feather groups was also reduced in every case with the exception of the secondary feathers at $\alpha=40^{\circ}$. However, the coverts and secondary coverts reacted much differently.

The frequency of the secondary feathers located closer to the trailing edge of W2 increased by up to $40 \%$ when the angle of attack was $0^{\circ}$ or less. Surprisingly, the frequency of the covert feathers located closer to the leading edge was inhibited and the amplitude decreased at these same angles of attack. However, when the secondary covert feathers were inhibited at $\alpha$ $=40^{\circ}$, the frequency of the coverts on $\mathrm{W} 2$ increase by $10 \%$. 
The feather motion differences between W1 and W3 can be seen in Table 4. Unlike the application of hair spray, applying polyurethane to the wing did not cause any increases in feather amplitude or frequency. Covert and secondary covert feather motion was completely inhibited at every angle of attack.

Table 4: Feather Motion Reductions Caused by the Application of Polyurethane Spray

\begin{tabular}{|c|c|c|c|c|}
\hline \multicolumn{5}{|c|}{ W1 VS. W3 } \\
\hline $\boldsymbol{\alpha}\left(^{\circ}\right.$ ) & Head (in. H2O) & Feather Type & Frequency \% Decrease & Amplitude \%Decrease \\
\hline-40 & 0.125 & Primaries & $76 \%$ & $76 \%$ \\
\hline-40 & 0.125 & Secondaries & $100 \%$ & $100 \%$ \\
\hline-40 & 0.125 & Coverts & $0 \%$ & $0 \%$ \\
\hline-40 & 0.125 & Secondary Coverts & $100 \%$ & $100 \%$ \\
\hline 0 & 0.125 & Primaries & $91 \%$ & $0 \%$ \\
\hline 0 & 0.125 & Secondaries & $91 \%$ & $94 \%$ \\
\hline 0 & 0.125 & Coverts & $100 \%$ & $100 \%$ \\
\hline 0 & 0.125 & Secondary Coverts & $100 \%$ & $100 \%$ \\
\hline 40 & 0.125 & Primaries & Not Available & Not Available \\
\hline 40 & 0.125 & Secondaries & Not Available & Not Available \\
\hline 40 & 0.125 & Coverts & $100 \%$ & $100 \%$ \\
\hline 40 & 0.125 & Secondary Coverts & $0 \%$ & $0 \%$ \\
\hline
\end{tabular}

Primary and secondary feather motion data was only available when $\alpha$ was $-40^{\circ}$ and $0^{\circ}$. At $\alpha=-40^{\circ}$, secondary feather motion was completely inhibited. Primary feather frequency and amplitude were both reduced by $76 \%$ when compared to $\mathrm{W} 1$ at this angle of attack. When $\alpha=$ $0^{\circ}$, the frequency of both the primary and secondary feathers on W3 were reduced by $91 \%$. Secondary feather amplitude decreased by $94 \%$ at $\alpha=0^{\circ}$. However, the amplitude of the primaries did not change when compared to W1 at this angle of attack. 


\subsection{Surface Geometry Variations Resulting from Model Preparations}

The feather motion analysis showed significant differences between each wing model. However, the effects of hair spray, polyurethane, and Plasti Dip on the surface geometry of the wings needed to be evaluated as well. Understanding the differences in the surface geometry allowed the effects of the surface geometry to be considered along with the effects of feather reduction. This helped to understand how the overall performance effects of feathers on a wing were affected by both surface geometry and feather movement.

Figure 53 shows that the distance between the barbs of a feather decreased significantly when hair spray was applied. The application of polyurethane to the same feather caused this distance to decrease even more. However, applying Plasti Dip to W4 caused this distance to increase.
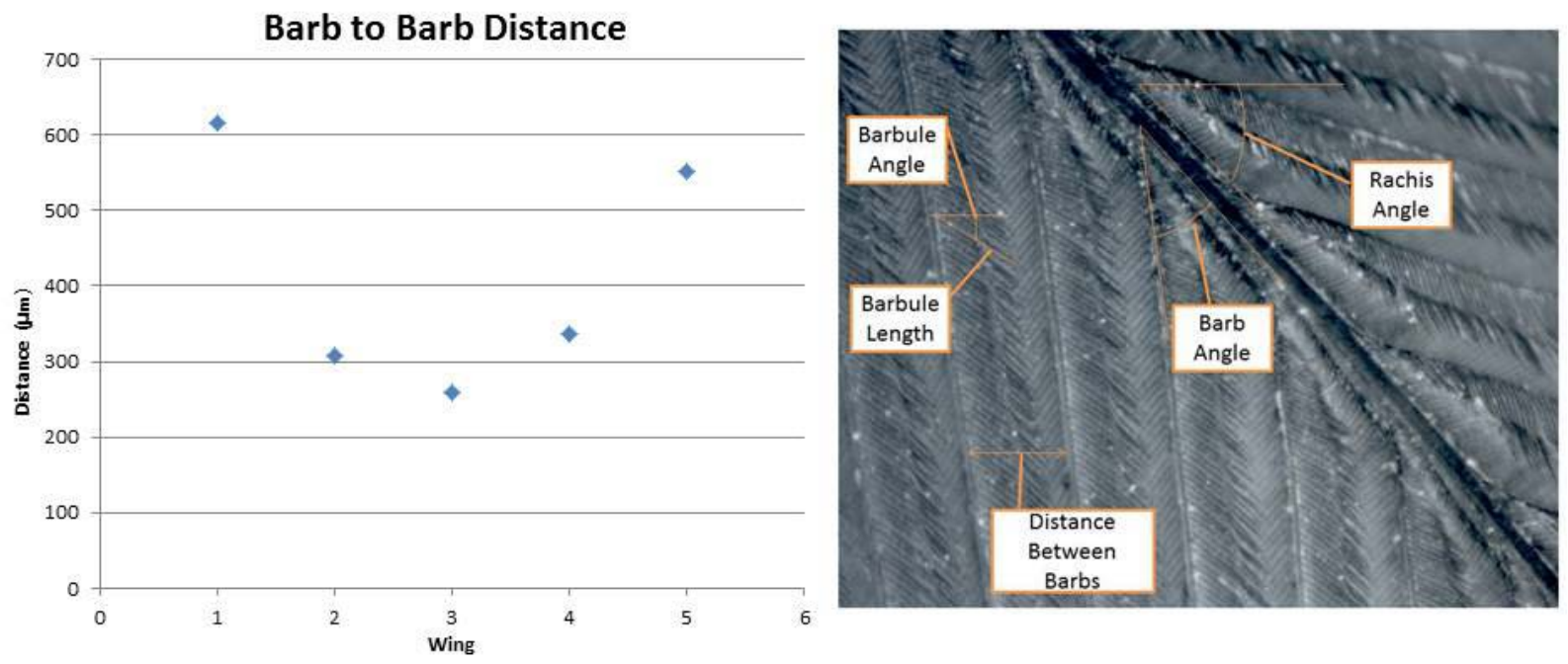

Figure 53: Variations Barb Spacing between Wing Models 
The results provided in Figure 54 show that the barb angle was also affected by each surface application. When hair spray was applied, the angle increased. However, the application of polyurethane caused the barb angle to decrease slightly. Applying Plasti Dip to W4 had almost no effect on the barb angle.
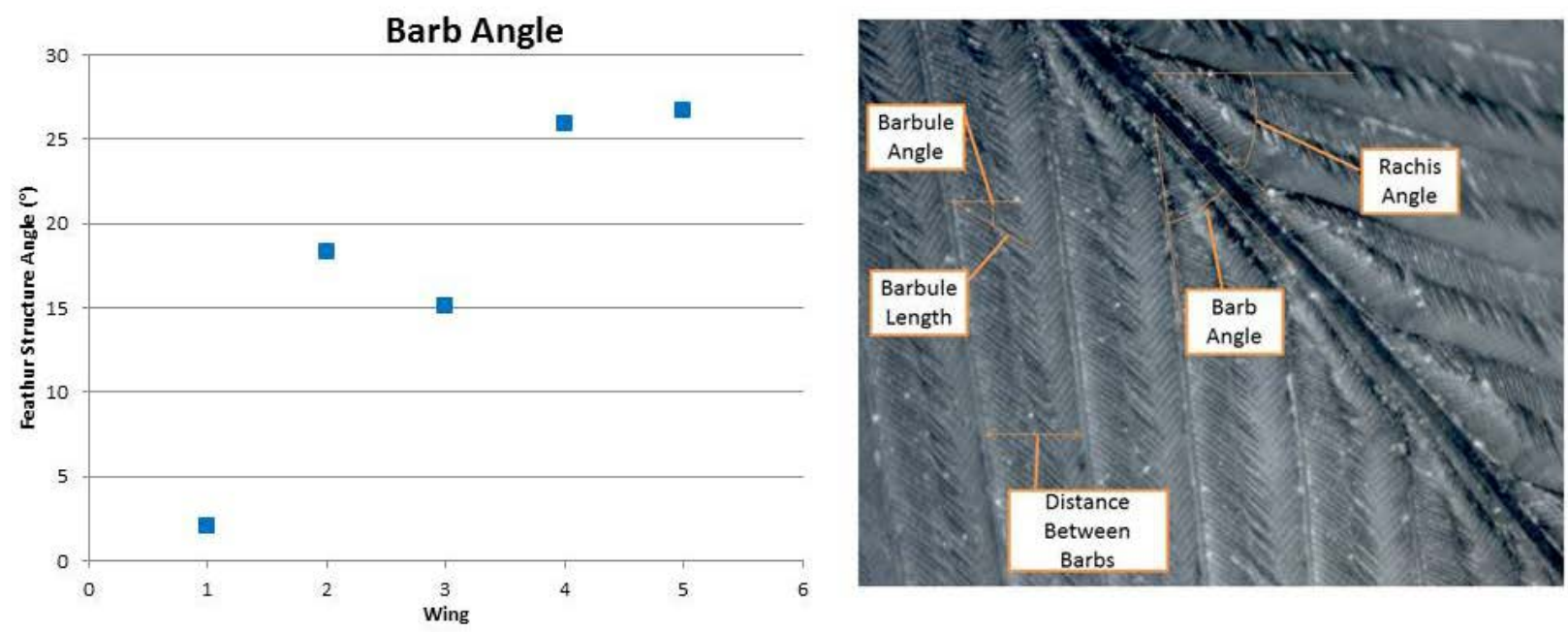

Figure 54: Variations in Barb Angle Between Wing Models

Figure 55 shows that the application of hair spray decreased the measured length of barbules. Applying polyurethane further decreased the length of the barbules. However, applying Plasti Dip to W4 increased the length of the barbules. 

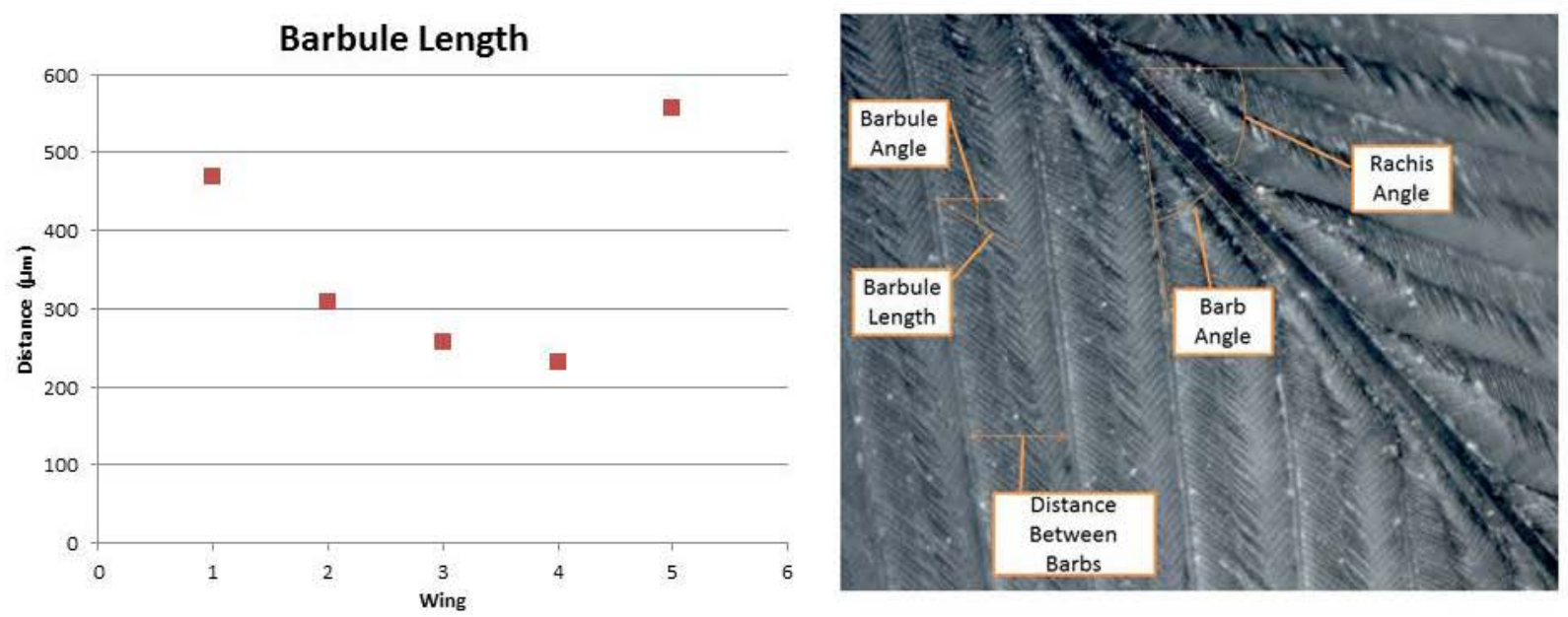

Figure 55: Changes in the Length of Barbules Between Wing Models

The application of hair spray caused the barbule angles of the feathers to increase as seen in Figure 56. The angle of the barbules was also affected by the application of polyurethane.

These changes in the angle of the barbs and barbules caused the features to retract closer to the larger structure to which they were attached.
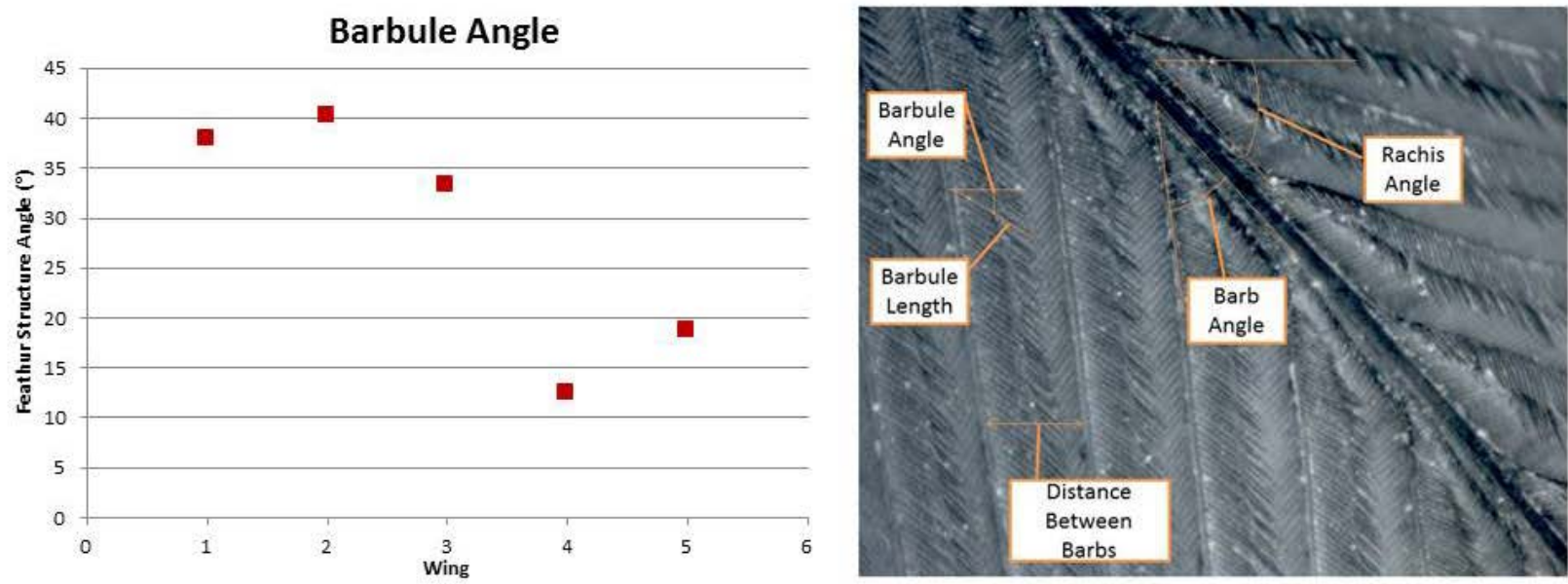

Figure 56: Differences in Barbule Angles Between Wing Models 
Figure 57 supports that the barbs and barbules retract closer to their respected base. This effect became less prominent when polyurethane was applied to the wing. However, applying polyurethane drastically reduced the depth of the surface features. As a result the surface of W3 was much smoother than the other wing models. The application of Plasti Dip to W4 also reduced the depth of this gap by a smaller amount.

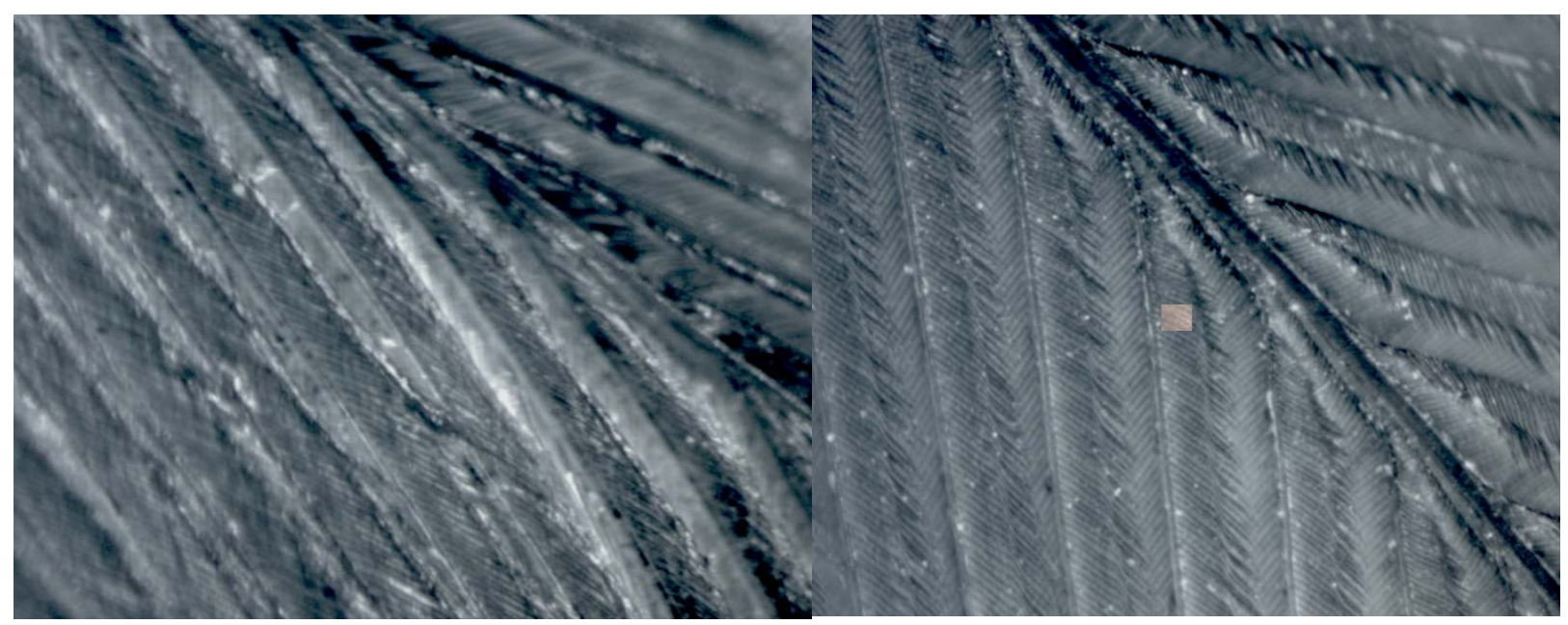

\section{Figure 57: The Changes in Barb and Barbule angles between the Natural (left) and Hair Sprayed (right) Wing Models}

Although hair spray affected the orientation of the barbs and barbules, Figure 58 shows that it had little effect on the depth of the surface features. On the other hand applying polyurethane drastically decreased this depth. Applying Plasti Dip to W4 also decreased the depth of the surface features, but the change was less severe. 


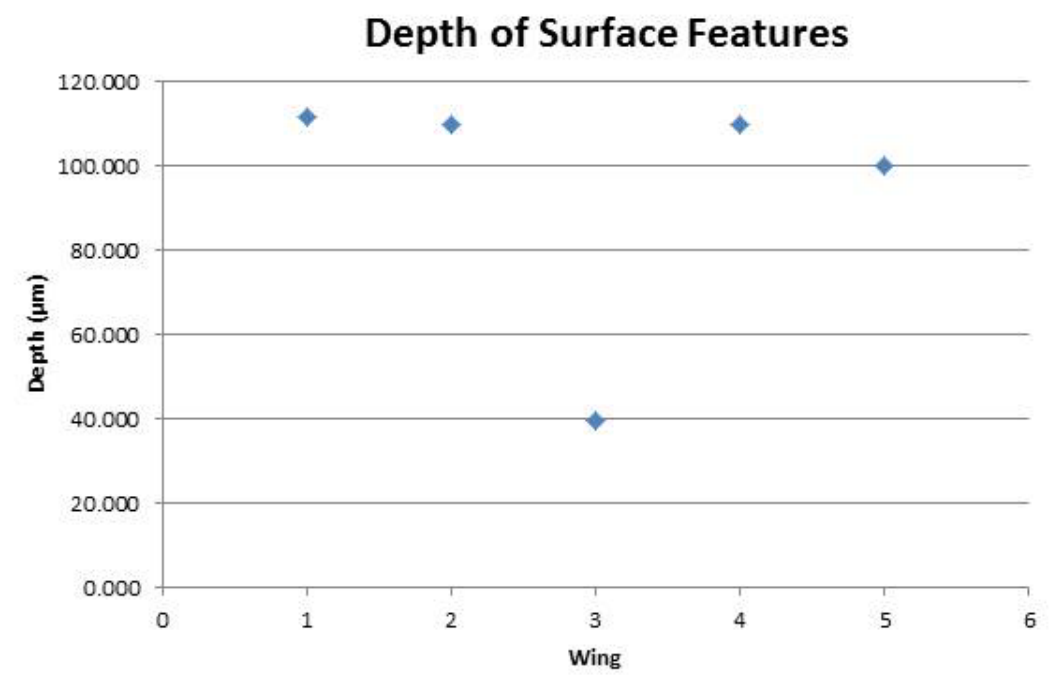

Figure 58: Variations in the Depth of Surface Features Between Wing Models

Applying polyurethane reduced the depth of surface features of W3 by 65\% when compared to W1. The application of Plasti Dip to W4 was expected to have a similar effect. Table 5 shows that the application of polyurethane caused the largest changes in most of the surface geometry of the natural wing. 
Table 5: Measured Changes in Surface Geometry between Wing Models

\begin{tabular}{|c|c|c|c|c|c|c|c|c|c|c|c|c|}
\hline \multirow{2}{*}{$\begin{array}{l}\text { Measurement } \\
\text { Barb Angle ( } \beta \text { ) }\end{array}$} & \multicolumn{2}{|c|}{ Wing 1 vs Wing 2} & \multicolumn{2}{|c|}{ Wing 1 vs Wing 3} & \multicolumn{2}{|c|}{ Wing 2 vs Wing 3} & \multicolumn{2}{|c|}{ Wing 4 vs Wing 5} & \multicolumn{2}{|c|}{ Wing 4 vs Wing 3} & \multicolumn{2}{|c|}{ Wing 5 vs Wing 3} \\
\hline & $89.0 \%$ & Increase & $86.7 \%$ & Increase & $17.6 \%$ & Decrease & $3.0 \%$ & Increase & $18.4 \%$ & Decrease & $33.0 \%$ & Decrease \\
\hline $\begin{array}{c}\text { Barb to Barb } \\
\text { Distance (Ъ) }\end{array}$ & $50.2 \%$ & Decrease & $57.8 \%$ & Decrease & $15.3 \%$ & Decrease & $58.3 \%$ & Increase & $22.6 \%$ & Decrease & $53.4 \%$ & Decrease \\
\hline $\begin{array}{c}\text { Barbule Length } \\
\text { (Г) }\end{array}$ & $34.0 \%$ & Decrease & $45.1 \%$ & Decrease & $16.8 \%$ & Decrease & $58.3 \%$ & Increase & $10.8 \%$ & Increase & $54.0 \%$ & Decrease \\
\hline $\begin{array}{c}\text { Barbule Angle } \\
\text { (3) }\end{array}$ & $53.6 \%$ & Decrease & $30.9 \%$ & Decrease & $32.9 \%$ & Increase & $23.8 \%$ & Increase & $55.1 \%$ & Decrease & $66.2 \%$ & Decrease \\
\hline $\begin{array}{c}\text { Barbule to } \\
\text { Barbule } \\
\text { Distance }(\lambda)\end{array}$ & $45.8 \%$ & Decrease & $33.3 \%$ & Decrease & $18.8 \%$ & Increase & Not A & vailable & $100.0 \%$ & Increase & Not & ailable \\
\hline $\begin{array}{c}\text { Depth of } \\
\text { Surface } \\
\text { Features ( } \delta)\end{array}$ & $1.5 \%$ & Decrease & $64.7 \%$ & Decrease & $64.1 \%$ & Decrease & $8.6 \%$ & Decrease & $64.1 \%$ & Decrease & $60.7 \%$ & Decrease \\
\hline
\end{tabular}

As seen in Figure 59, W5 also had a smother surface than W4as a result of the Plasti Dip. However, unlike W3, only the gaps between the barbs and barbules were filled in. The $\delta$ remained within $8.5 \%$ of W4. Therefore, only the smaller, less prominent surface structures, such as the barbules were smoothed over. 


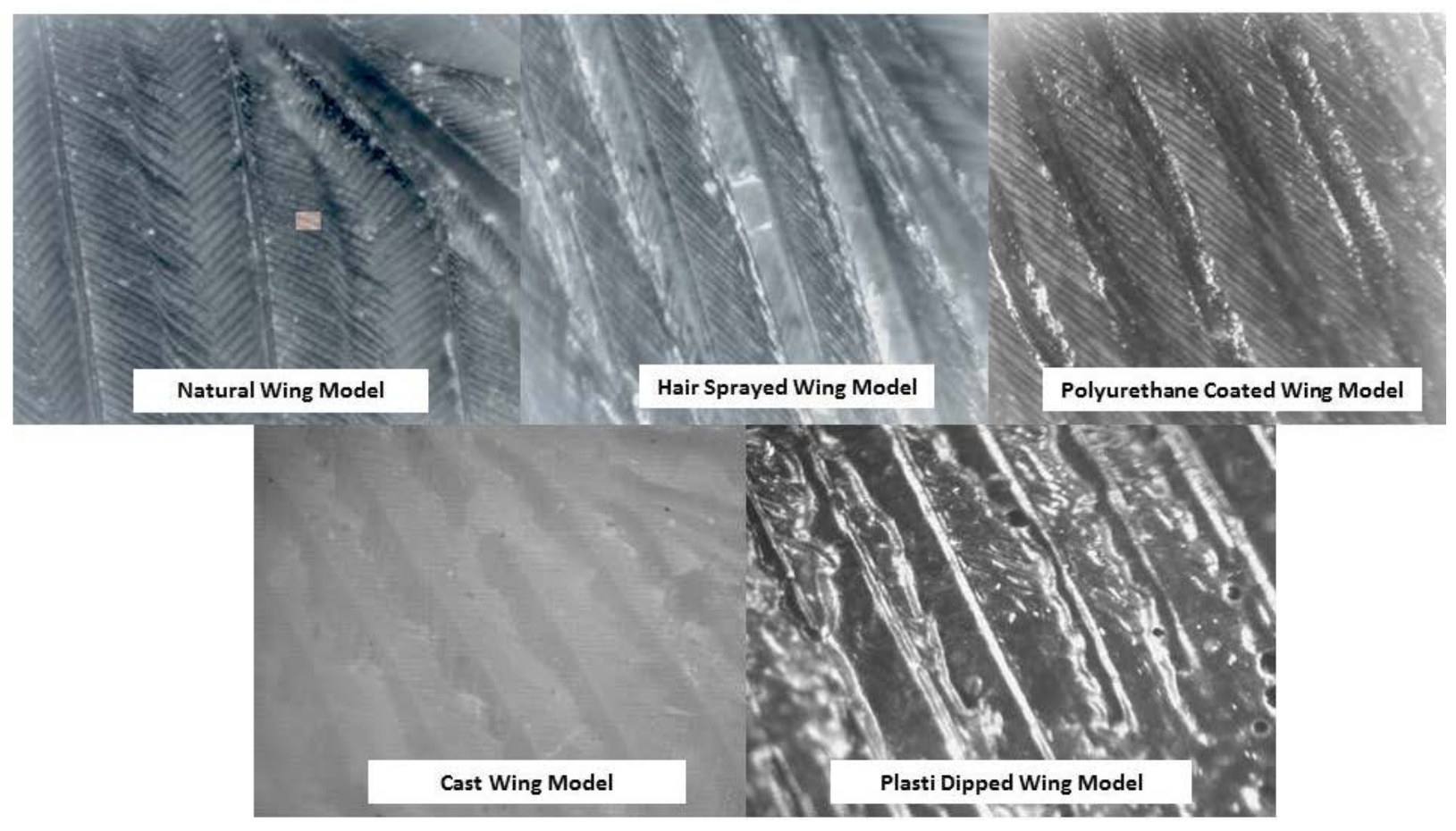

Figure 59: A Comparison of the Surfaces of the Cast, Plasti Dipped, Natural, Hair Sprayed and Polyurethane Coated Wing Models 


\subsection{Influence of Treatment on Feather Material Properties}

In order to quantify the effects of treating feathers with hair spray and polyurethane on their material properties several tests were completed using individual feathers. The resonant frequencies of seven covert feathers in their natural, hair sprayed, and polyurethane coated forms were found by attaching the individual feathers to a shaker table and varying the frequency until the maximum feather displacement was found. In order to imitate the feathers of on the hair sprayed and polyurethane coated wings as closely as possible, the individual feathers used in these tests were coated with hair spray or polyurethane on only one side. Figure 60 shows that applying hair spray to the feathers reduced the average resonant frequency of the feathers. Applying polyurethane to the feathers further decreased the average resonant frequency of the feathers.

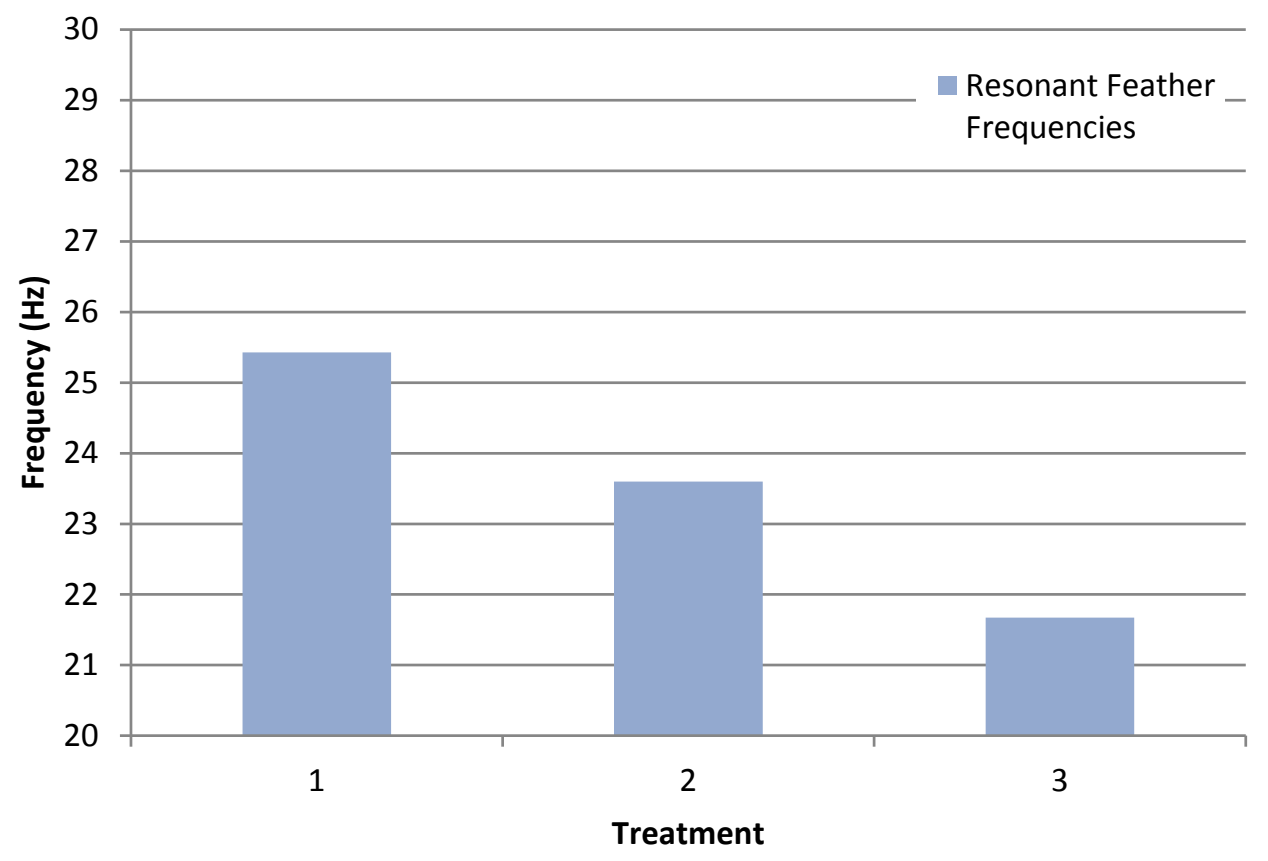

Figure 60: Average Resonant Frequencies the Test Feathers with Each Surface Treatment 
Further tests were conducted using the same seven covert feathers in a cantilever beam set up. The goal was to find the deflection caused by a $0.0172 \mathrm{lb}$. load applied six inches from the base of the feather. The results of these tests are shown in Figure 61. By applying hair spray, the average deflection of the feathers increased when compared to the preserved feather. Applying polyurethane caused the average deflection to further increase.

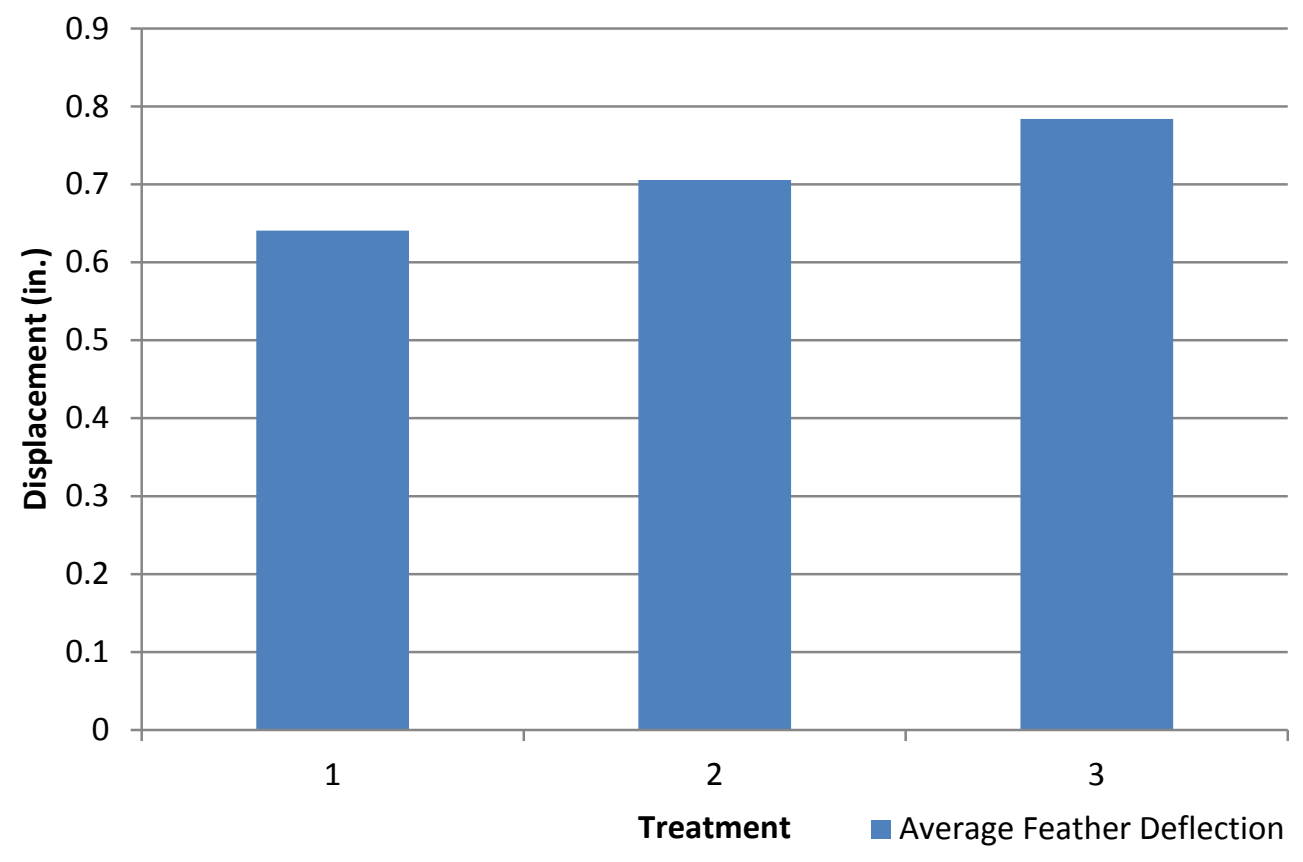

Figure 61: The Deflection of Feathers of Each Treatment Caused by a Known Load

The weight of the feathers was also measured after each application. As expected, Figure 62 shows that each treatment increased the weight of the feathers incrementally. On average the application of hair spray increased the mass of the feathers by $11 \%$ when compared to the untreated feathers. Applying polyurethane further increased the weight of the hair sprayed feathers by $18 \%$ when compared to the hair sprayed feathers or $40 \%$ when compared to the untreated feathers. 


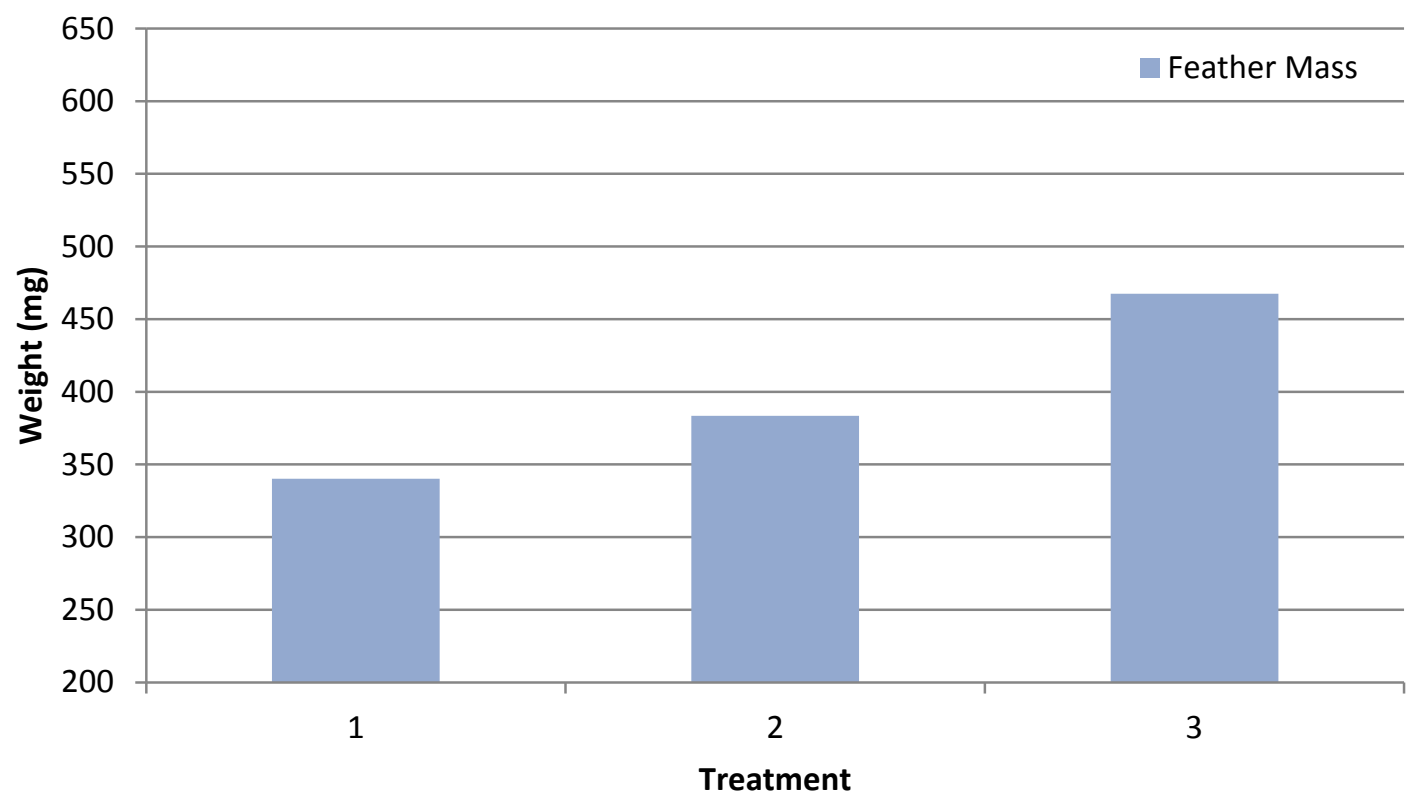

Figure 62: Feather Mass after Each Surface Treatment

Figure 63 shows that with each application the average modulus of elasticity of the feathers decreased. The application of hair spray to the untreated feathers caused the average modulus of elasticity to decrease by $14 \%$. Applying polyurethane to the hair sprayed feathers caused the average modulus of elasticity to decrease by $16 \%$ or $27 \%$ when compared to the untreated feathers. The modulus of elasticity of red tailed hawk feathers was not available for comparison. However, it was found that the average modulus of elasticity of a sparrow hawk or American kestrel feather is $3.5 \times 10^{9} \mathrm{psi}$ [27]. The calculated average modulus of elasticity of the untreated feathers used for this experiment was found to be $3.1 \times 10^{9}$. This resulting $12.9 \%$ difference between the two feathers was used to verify the calculated results. 


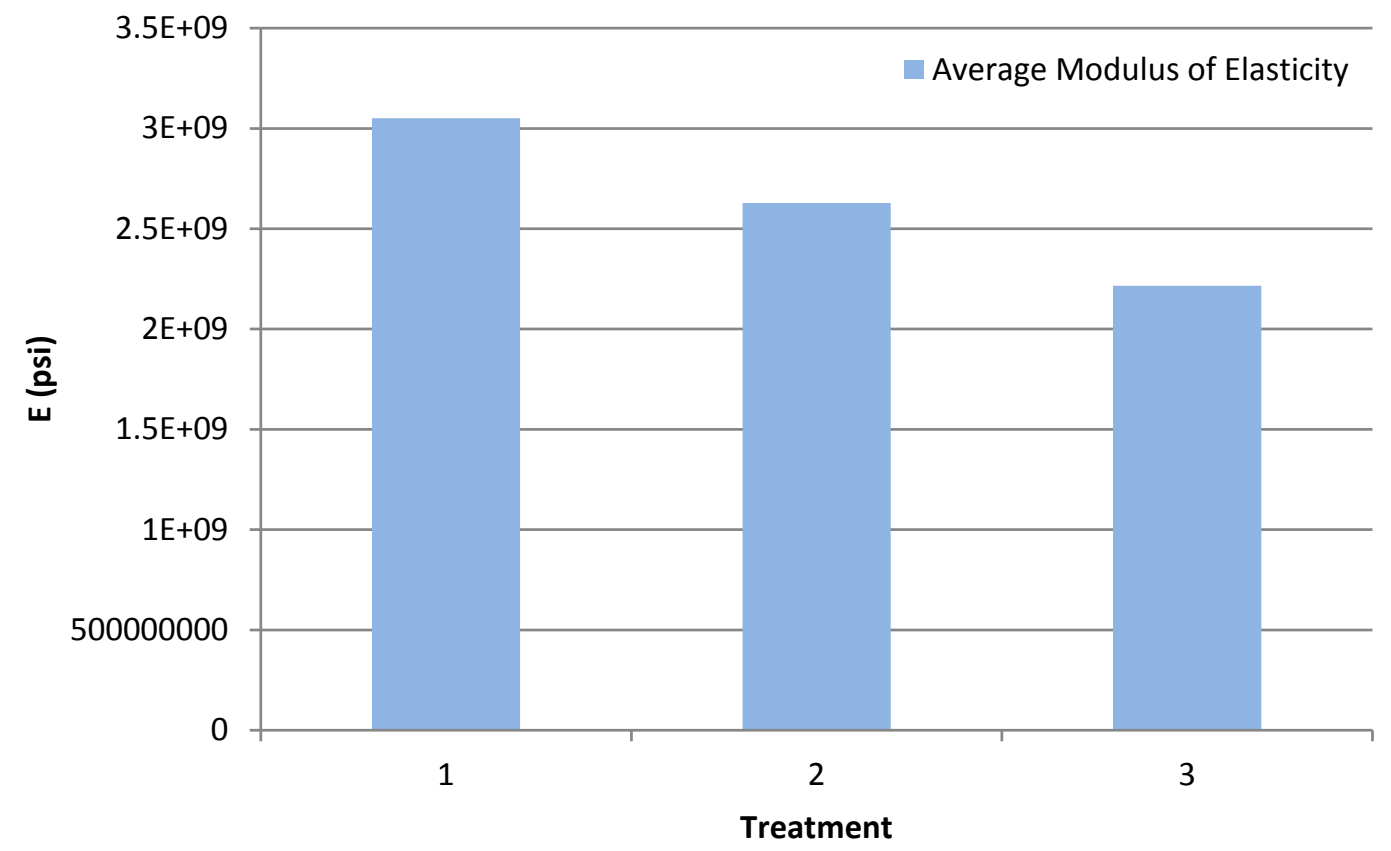

Figure 63: The Average Modulus of Elasticity of Tested Feathers

\subsection{Aerodynamic Force Data Results}

The impacts of feather motion were found through a comparative analysis of the aerodynamic force data collected from the five wing models. This analysis supports the completion of the objectives of this study to determine the effects of the feather motion and the influence of the surface geometry on the performance of a wing. Therefore, this analysis was split into the three sections to best explain the results; sensitivity to Reynolds number, impacts of feather motion, and effects of surface geometry. 


\subsubsection{Sensitivity to Reynold's Number}

The coefficient of lift $\left(C_{L}\right)$ curve of W1 was affected by the Reynold's number $(R e) . C_{L}$ decreased slightly as the $R e$ increased from 61,000 to 72,000 , then increased slightly as the $R e$ increased to 85,000 as seen in Figure 64 . When the $R e$ was increased to 78,000, a sudden increase in $C_{L}$ was noticed. This occurred between $12^{\circ}$ and $16^{\circ}$ angle of attack $(\alpha)$.

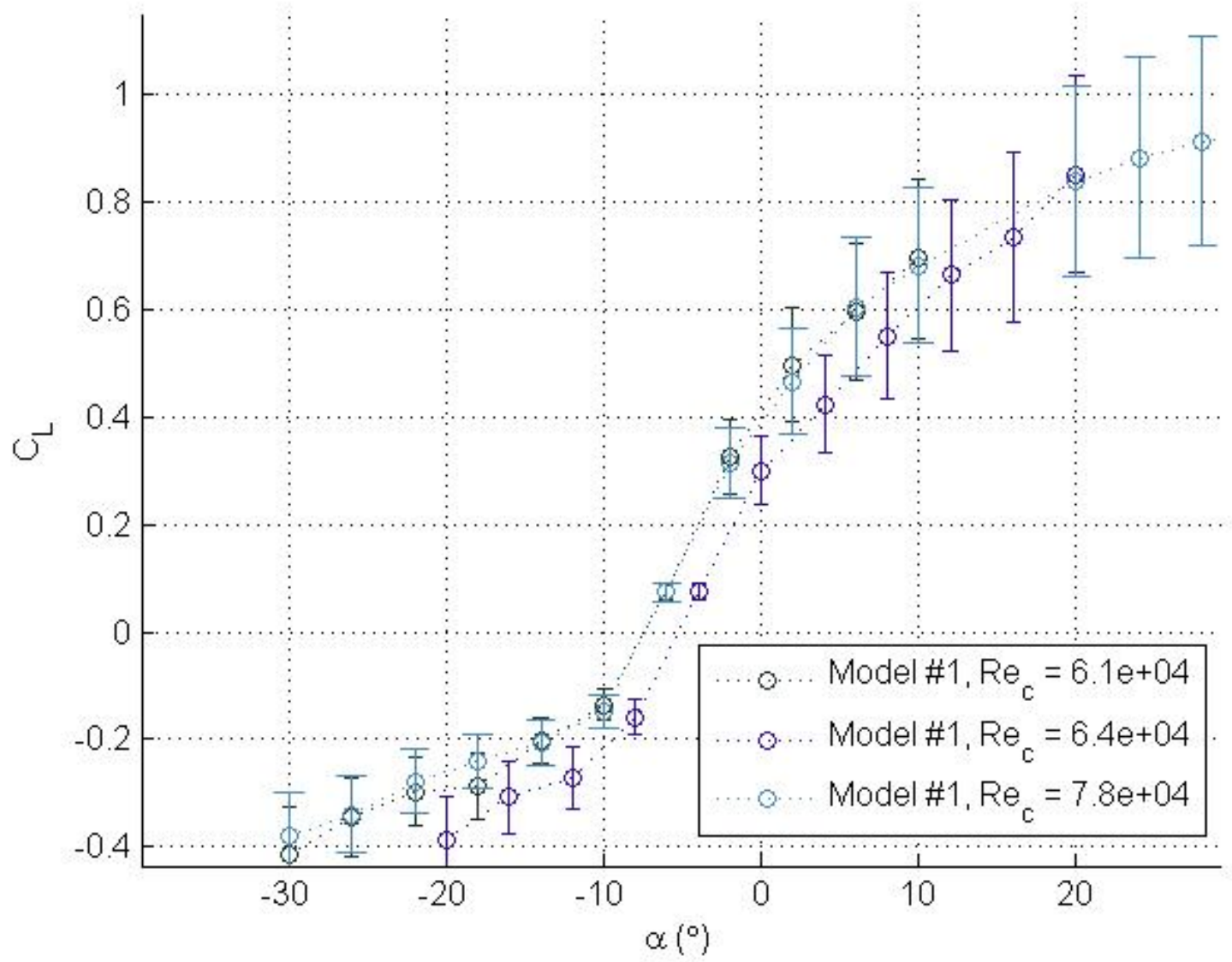

Figure 64: $C_{L}$ Sensitivity to Reynolds Number for the Preserved Wing Model Below 80,000 Re

Figure 65 shows that as $R e$ was increased beyond 78,000, the $C_{L}$ curve began to decrease. The curve continued to decrease as $R e$ was increased. Most of these changes in $C_{L}$ occurred within the uncertainty, except near $\alpha=-8^{\circ}$. 


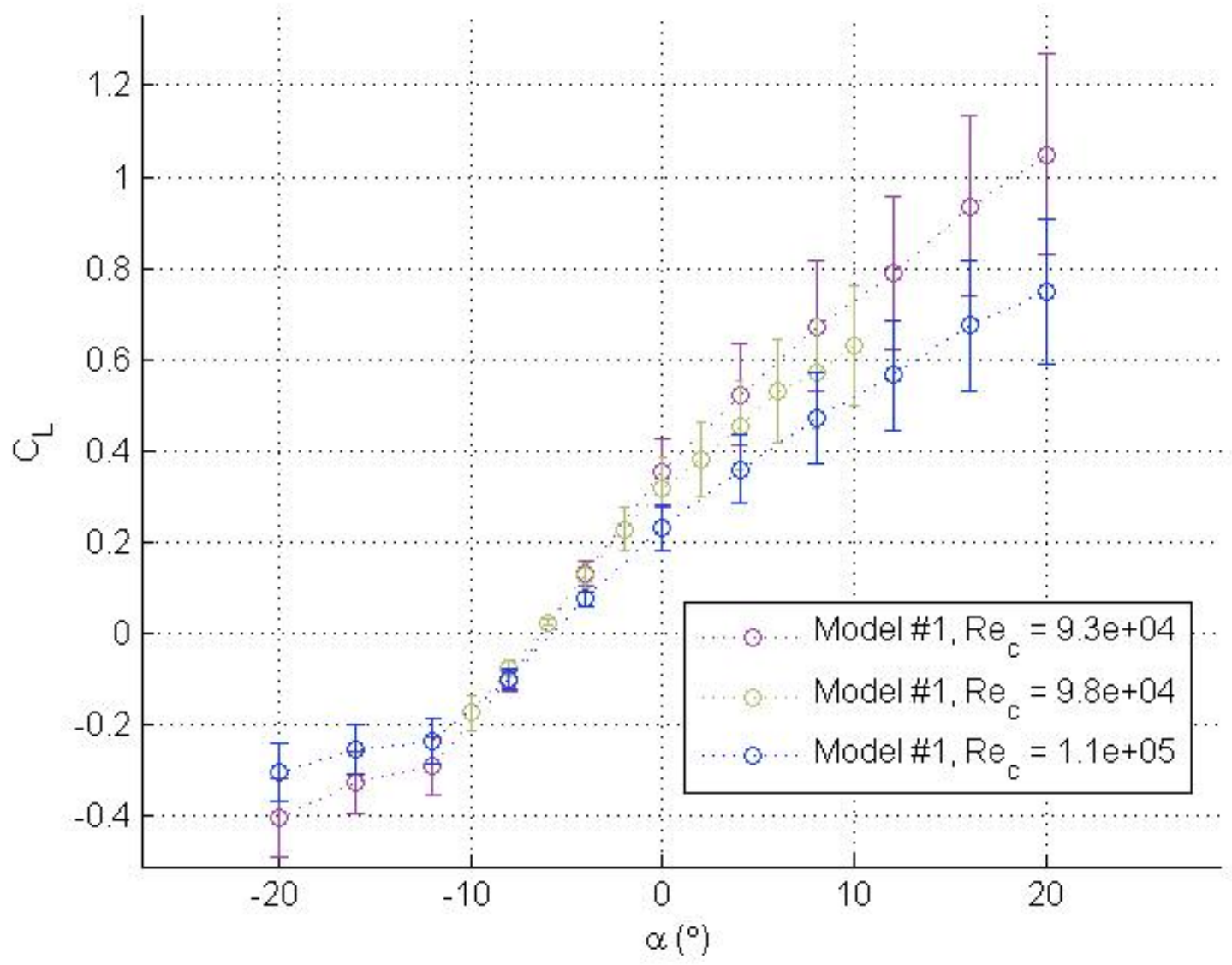

Figure 65: $C_{L}$ Sensitivity to Reynolds Number for the Preserved Wing Model Above 80,000 Re

$R e$ appeared to have less of an effect on the coefficient of drag $\left(C_{D}\right)$ between 61,000 and 78,000. Differences between the two curves were minimal. These small changes can be seen in Figure 66. 


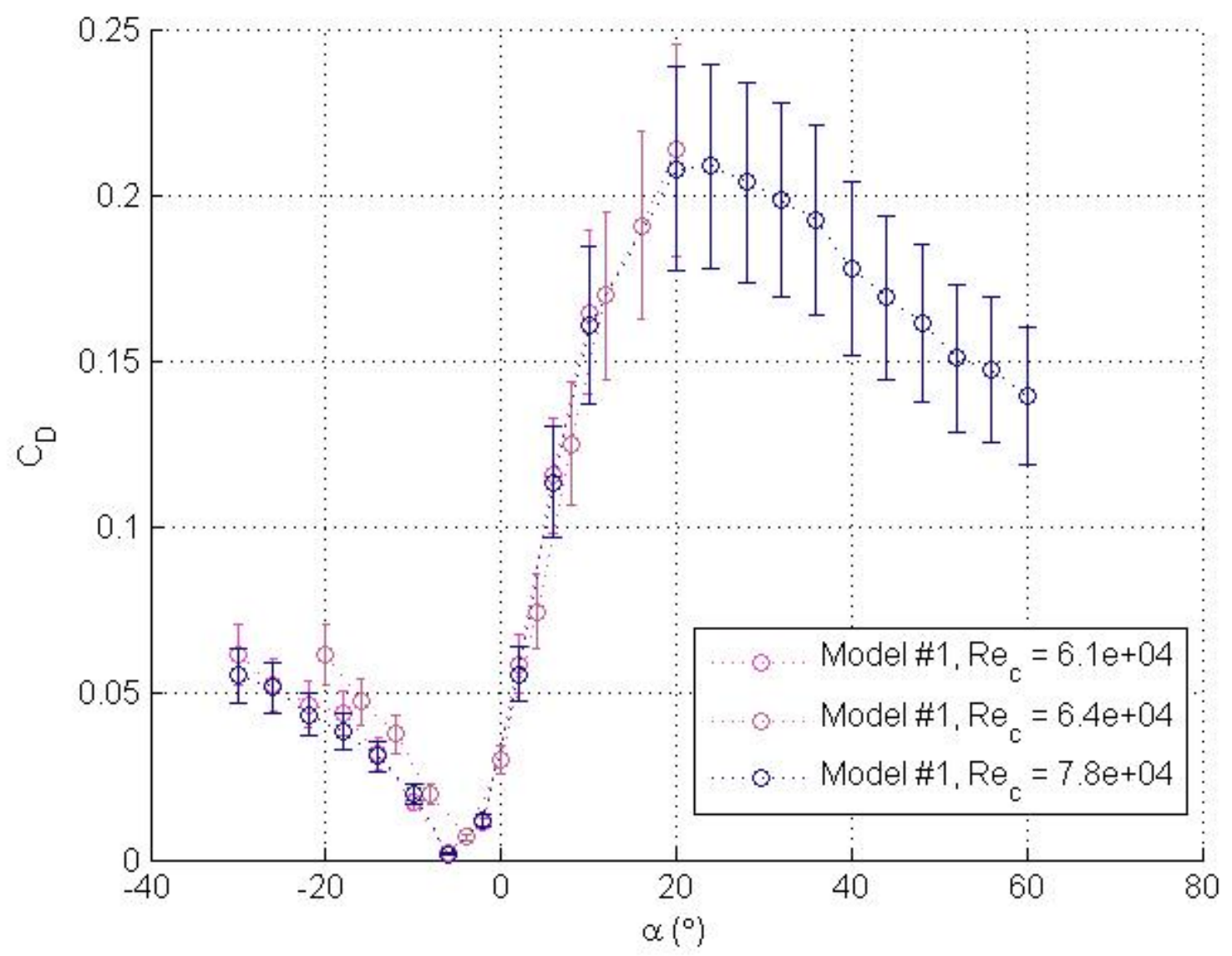

Figure 66: $C_{D}$ Sensitivity to Reynolds Number for the Preserved Wing Model Below 80,000 Re

Figure 67 shows the change in $C_{D}$ of W1 when $R e$ was increased above 80,000. However, the change in $C_{D}$ surpassed the uncertainty between $12^{\circ}$ and $20^{\circ} \alpha$. This occurred when $R e$ was increased from 85,000 to 105,000 . 


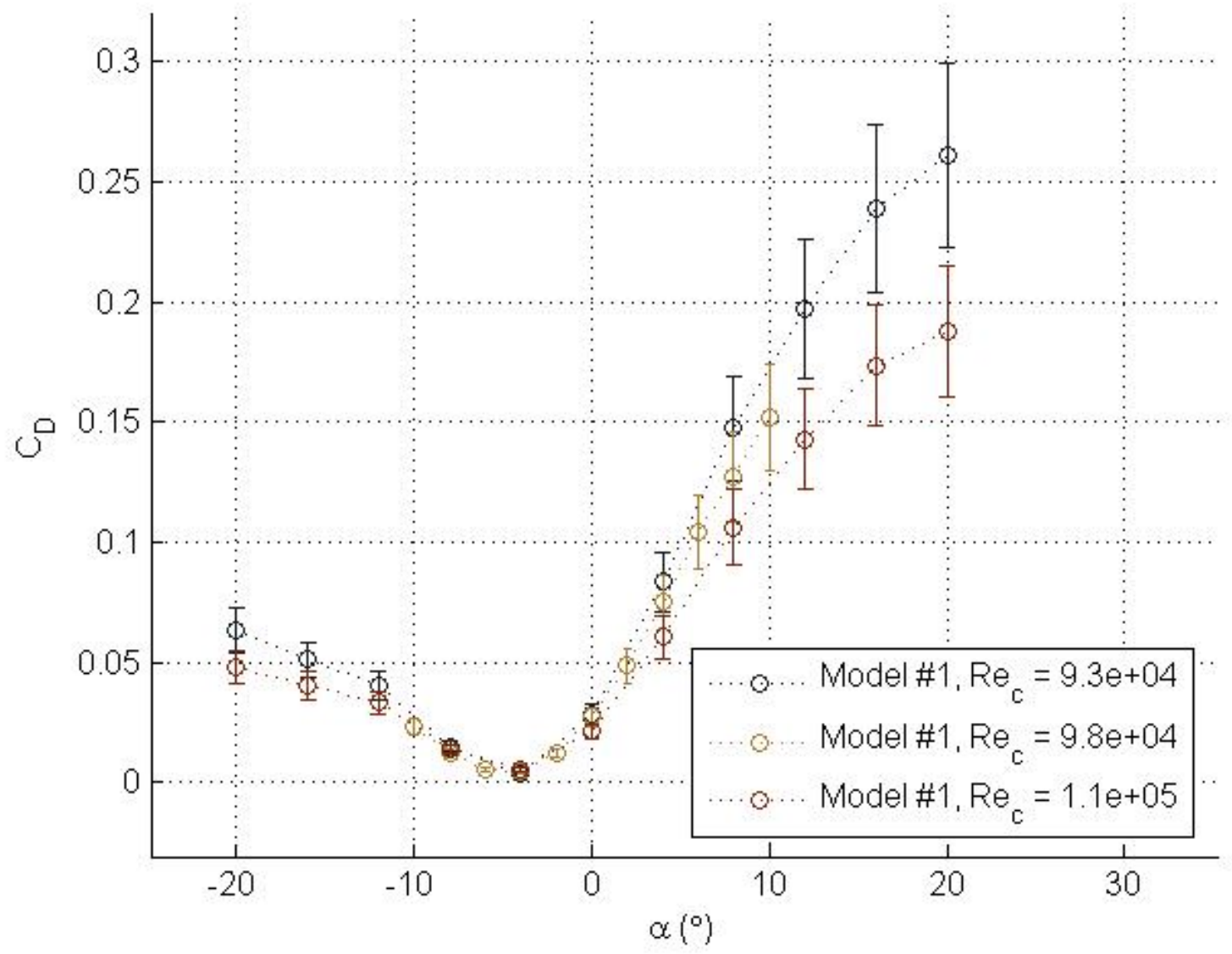

Figure 67: $C_{D}$ Sensitivity to Reynolds Number for the Preserved Wing Model Above 80,000 Re

Although some changes in $C_{L}$ occurred outside the uncertainty range near $\alpha=-8^{\circ}$, the same sensitivity to $R e$ was not exhibited by W2. Figure 68 shows that the $C_{L}$ curve of W2 was more constant than W1. Therefore, changing less throughout the Re test range. No sudden changes were observed. 


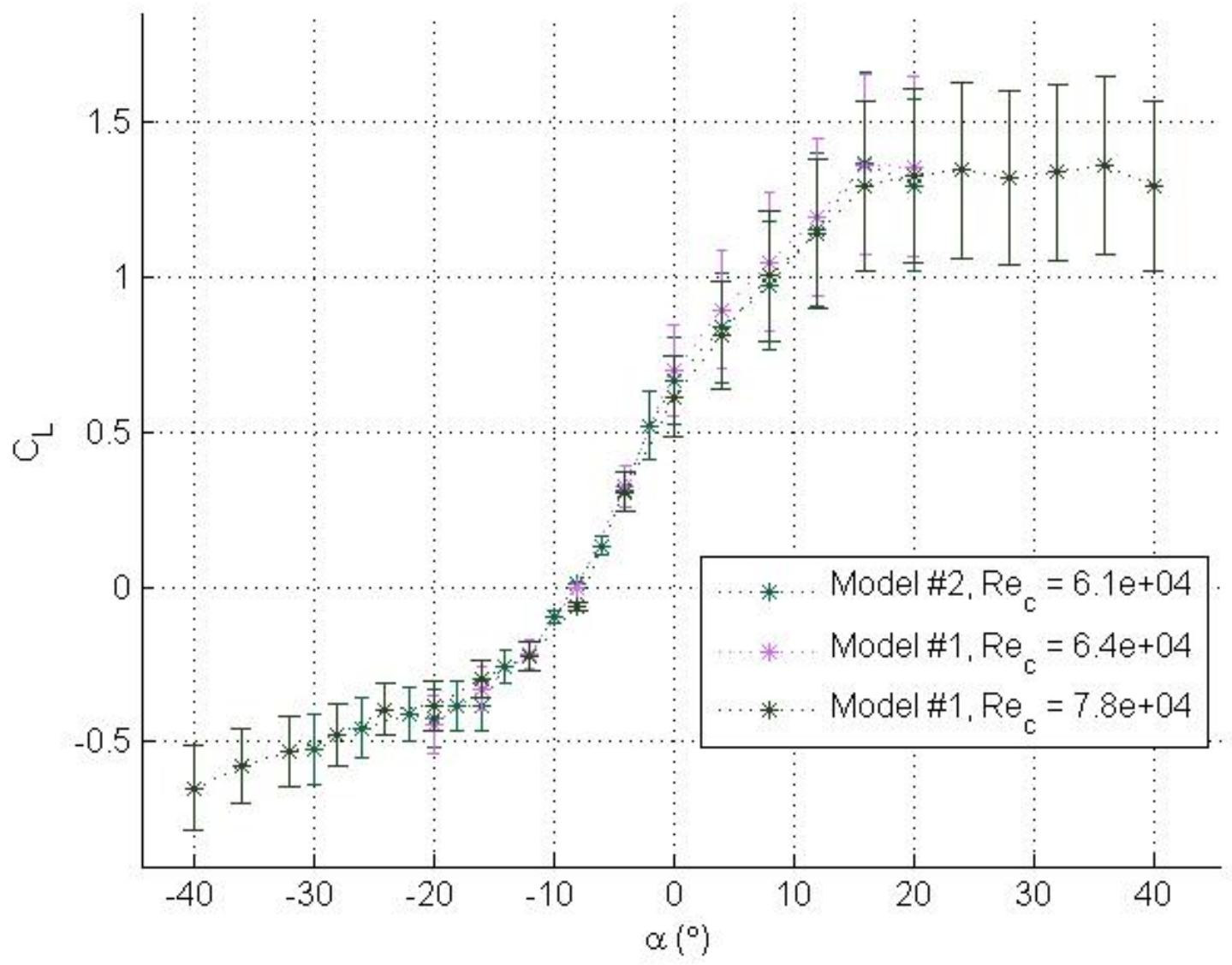

Figure 68: $C_{L}$ Sensitivity to Reynolds Number for the Hair Sprayed Wing Model Below 80,000 Re

The reduced influence of $C_{L}$ for W2 when compared to W1 can be seen in Figure 69. Some reduction in $C_{L}$ was observed at the $R e$ was increased above 80,000. However, this was still less of a change than W1. 


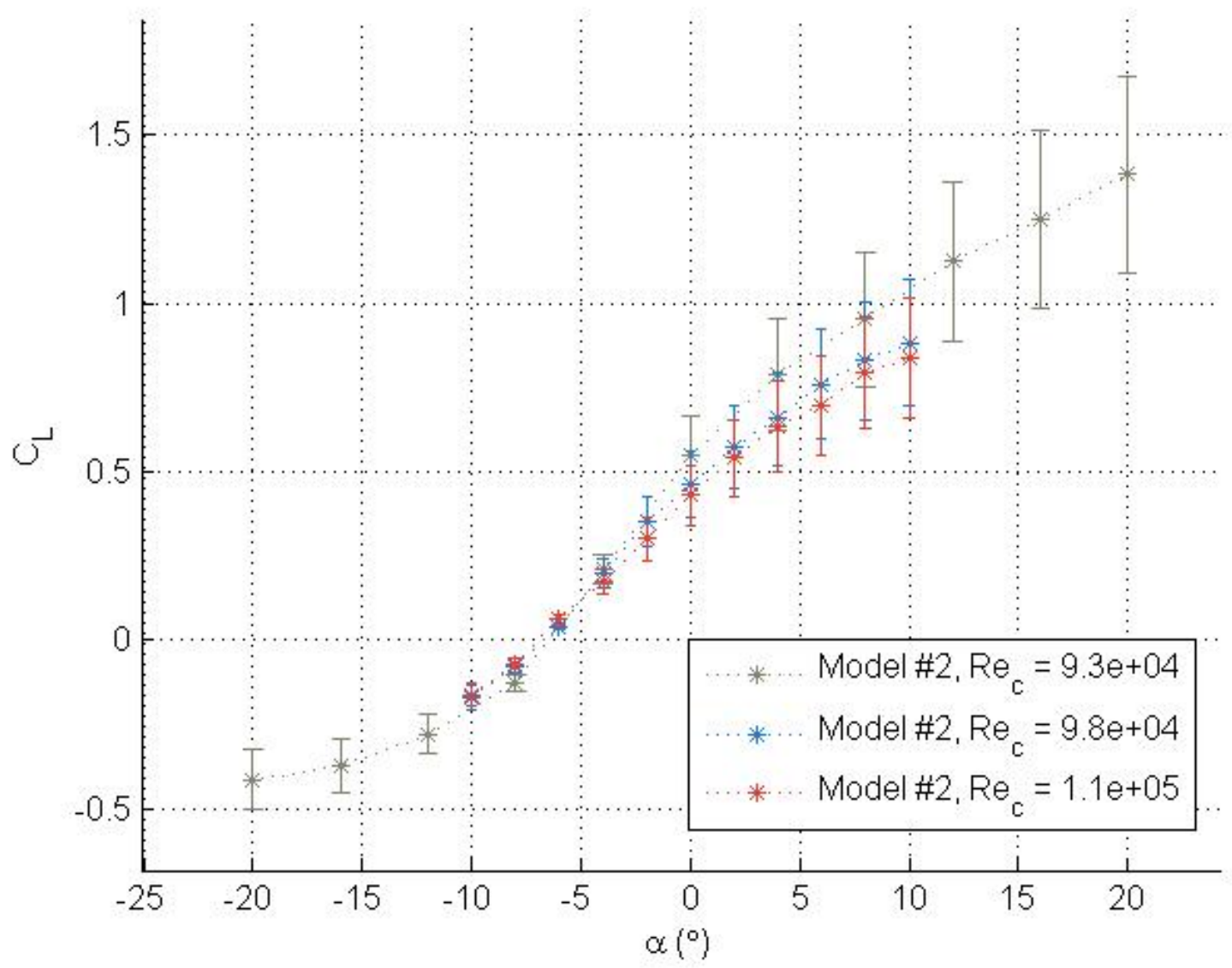

Figure 69: $C_{L}$ Sensitivity to Reynolds Number for the Hair Sprayed Wing Model Above 80,000 Re

Figure 70 shows that $C_{D}$ was also less affected by $R e$ for W2. Almost no differences between the $C_{D}$ curves of the wing below 80,000 Re were observed. Changes in $C_{D}$ for W2 were only outside of the uncertainty at $\alpha=0^{\circ}$ 


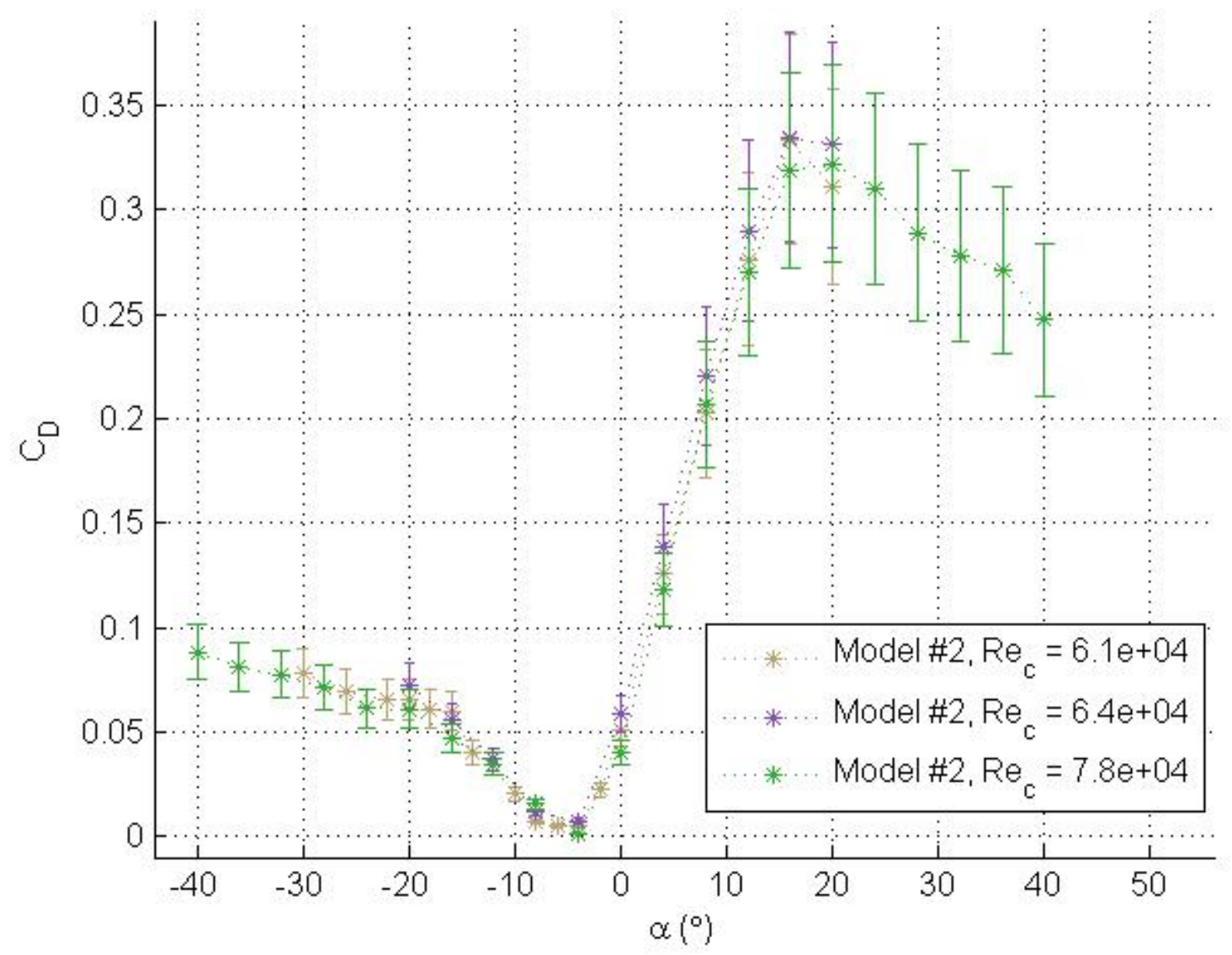

Figure 70: $C_{D}$ Sensitivity to Reynolds Number for the Hair Sprayed Wing Model Below 80,000 Re

As Re was increased above $80,000, C_{D}$ began to decrease as $R e$ was increased. This change can be seen in Figure 71. However, these decreases occurred at a lower angle of attack range than $\mathrm{W} 1$. 


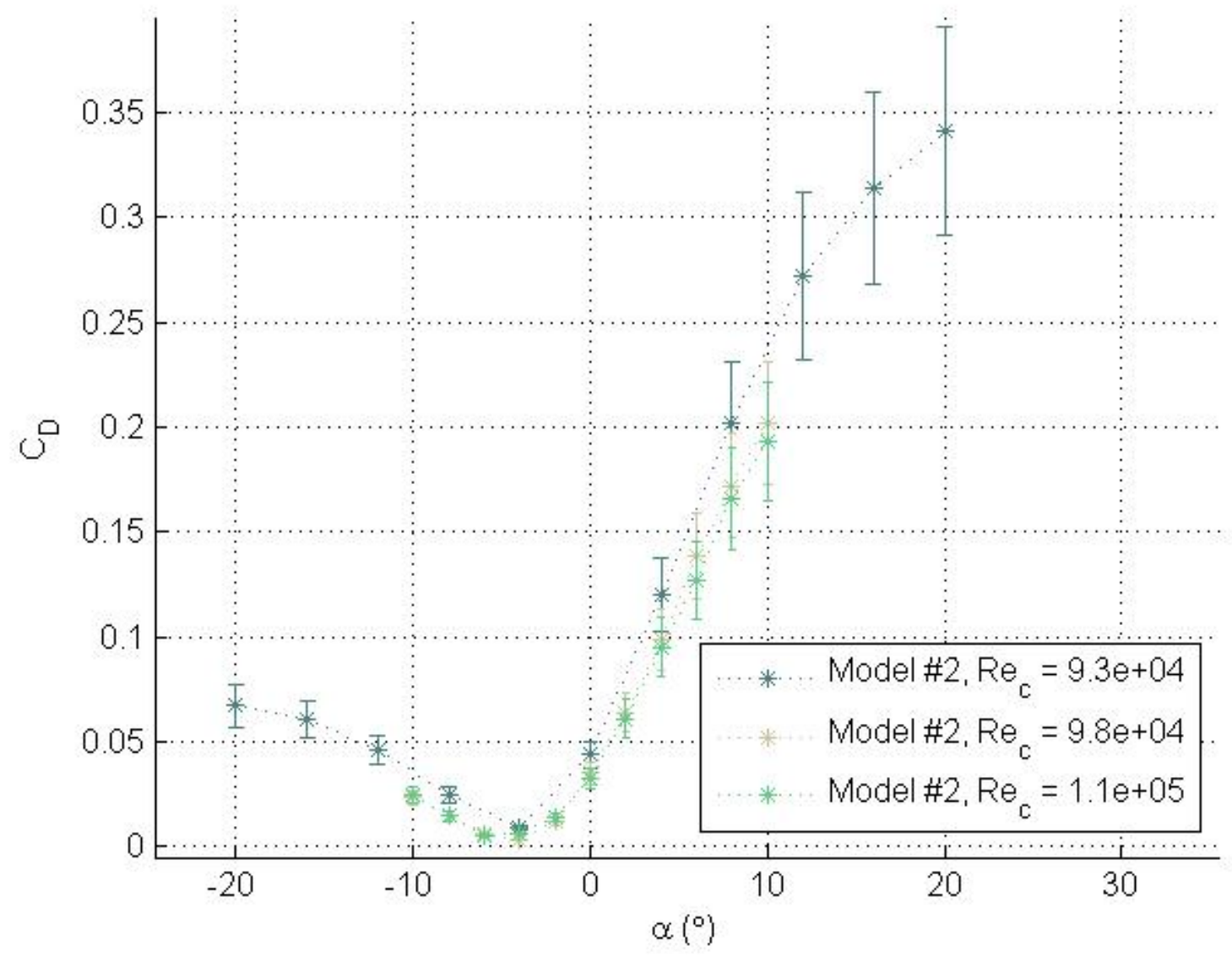

Figure 71: $C_{D}$ Sensitivity to Reynolds Number for the Hair Sprayed Wing Model Above 80,000 Re

$R e$ had even less effect on the performance of W3 than W2. Figure 72 shows changes in $C_{L}$ as the $R e$ was increased below 80,000. No fluctuation in $C_{L}$ occurred outside of the uncertainty. 


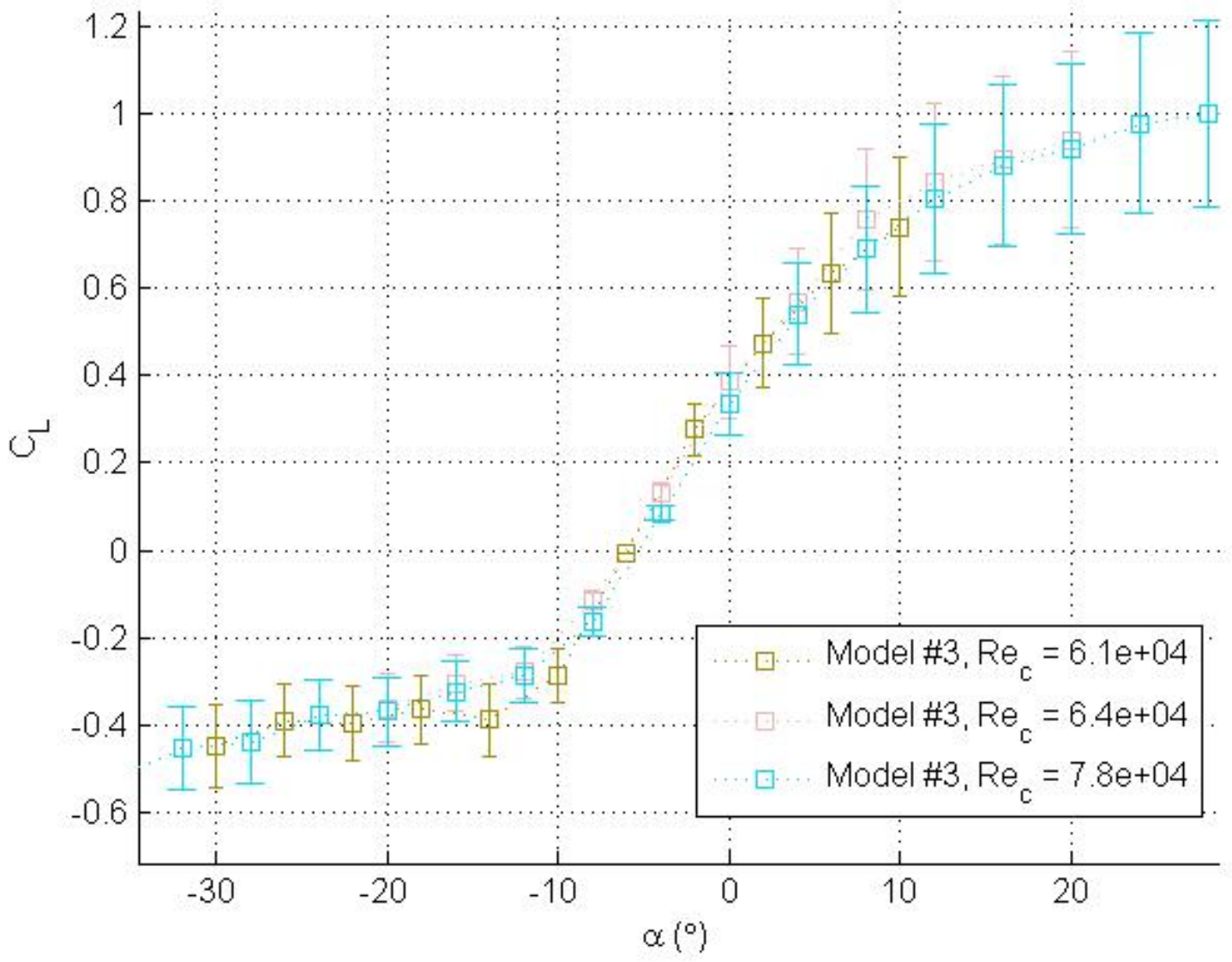

Figure 72: $C_{L}$ Sensitivity to Reynolds Number for the Polyurethane Coated Wing Model Below 80,000 Re

Figure 73 shows that as the Re was increased above 80,000, only small differences in the $C_{L}$ curves were found. However, none of these differences were outside of the uncertainty. The changes in $C_{L}$ for W3 continued to be less than those of both W1 and W2. 


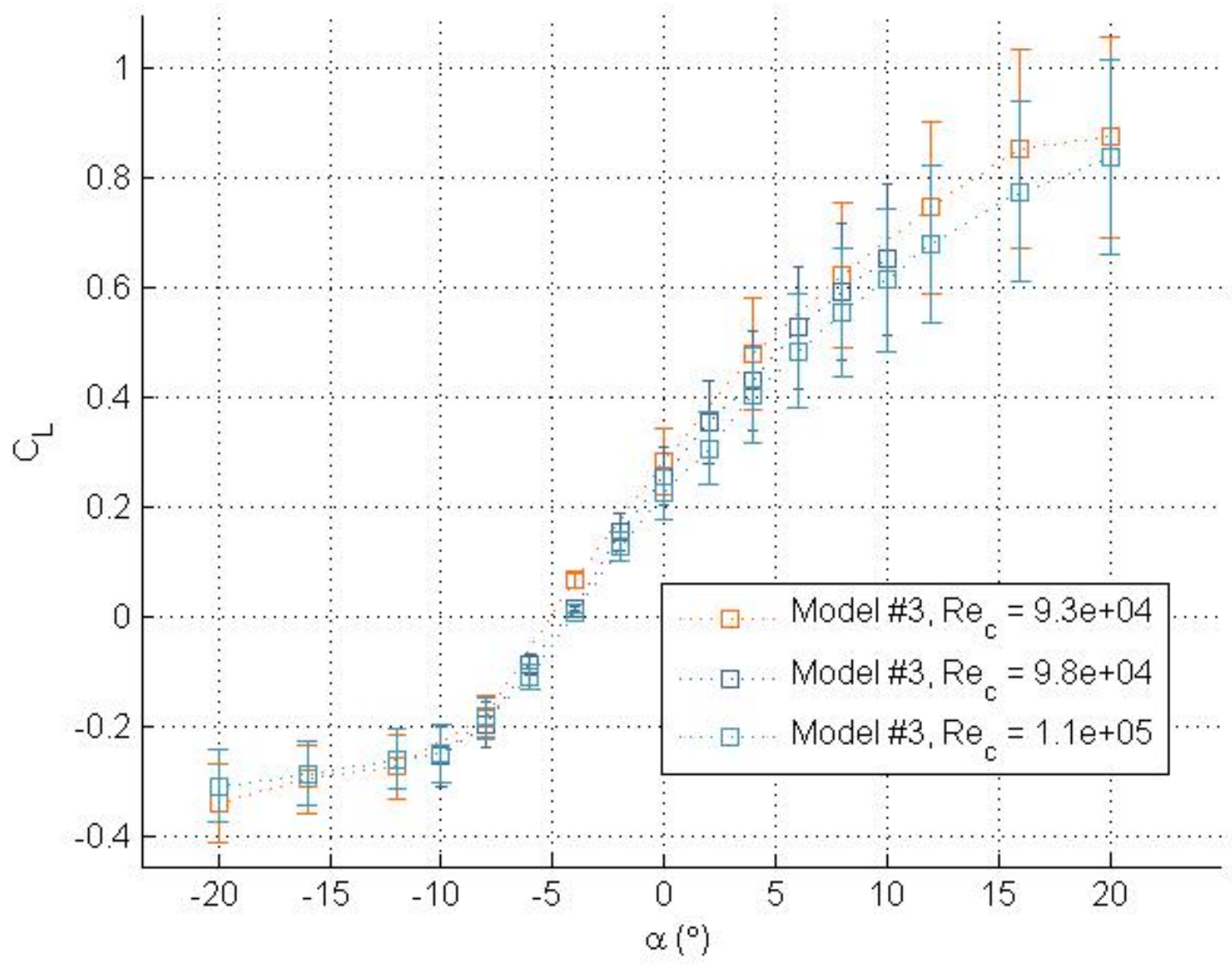

Figure 73: $C_{L}$ Sensitivity to Reynolds Number for the Polyurethane Coated Wing Model Above 80,000 Re

$C_{D}$ only changed outside of the uncertainty at $\alpha=-8^{\circ}$. When compared to W1 and W2, the performance of W3 was more consistent below 80,000 Re. No sudden changes occurred as with W1. 


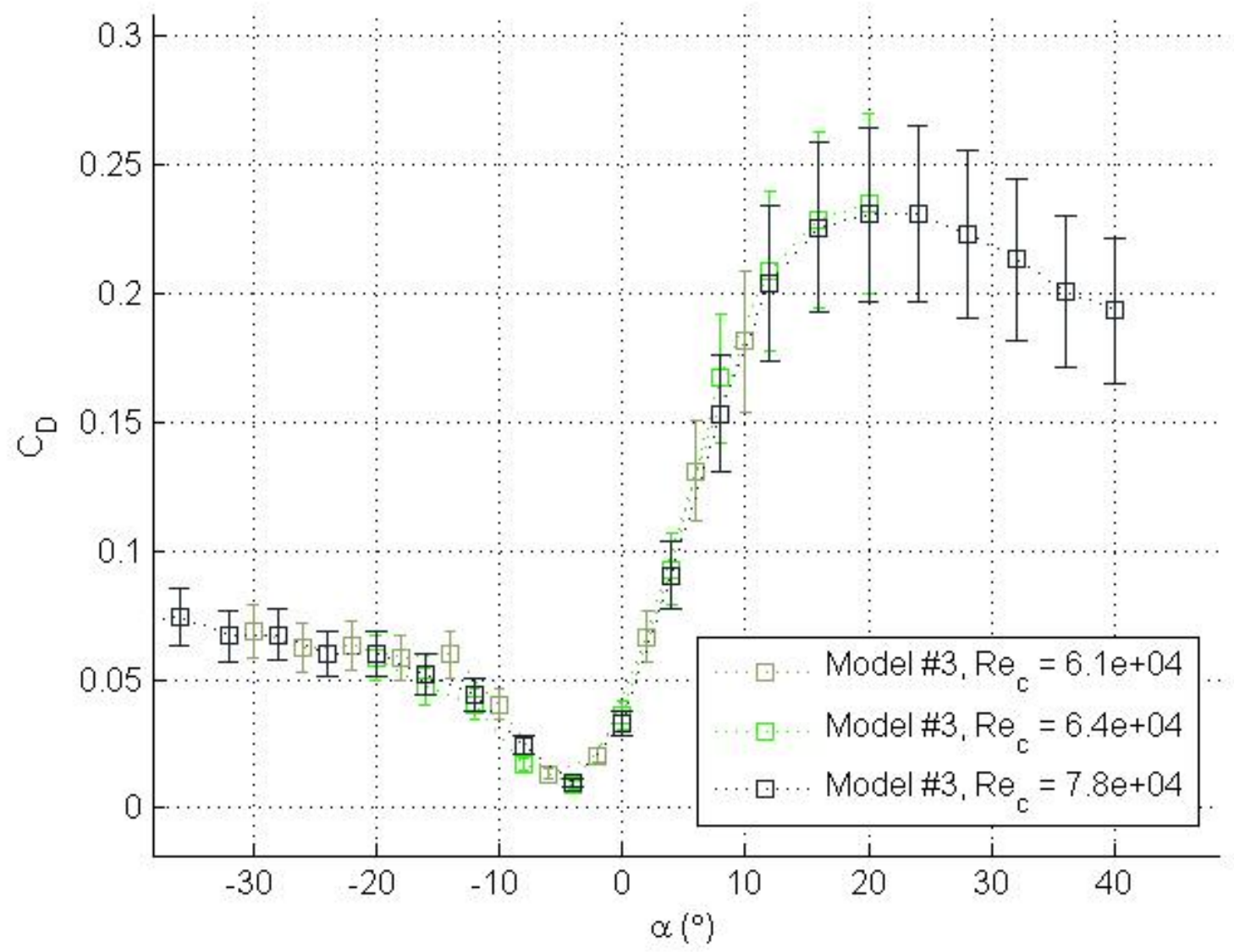

Figure 74: $C_{D}$ Sensitivity to Reynolds Number for the Polyurethane Coated Wing Model below 80,000 Re

The $C_{D}$ curves for W3 above 80,000 Re waivered little as the $R e$ was increased above 80,000. This can be seen in Figure 75. However, some decrease in $C_{D}$ occurred as the Re was increased. 


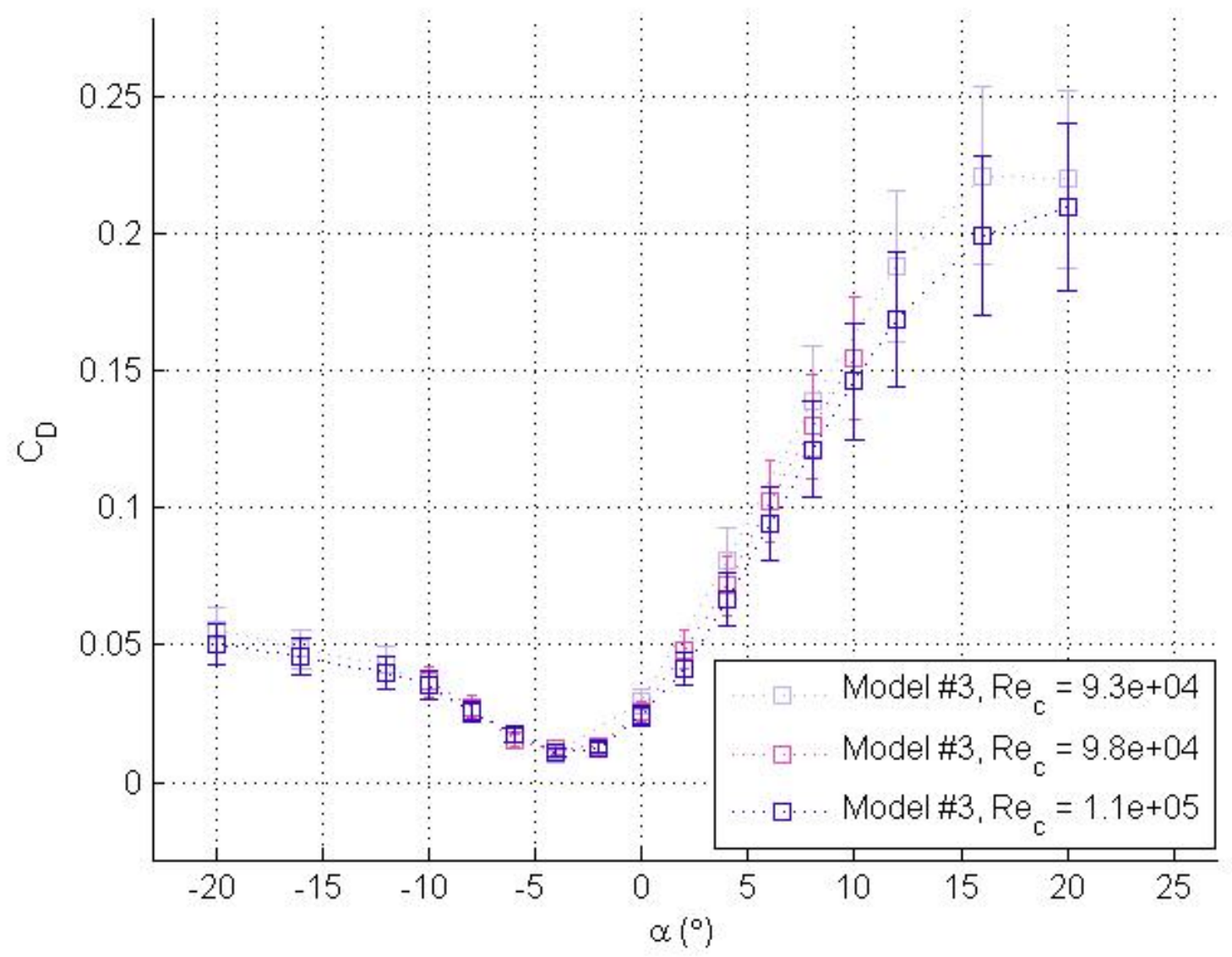

Figure 75: $C_{D}$ Sensitivity to Reynolds Number for the Polyurethane Coated Wing Model Above 80,000 Re

Figure 76 shows that $C_{L}$ of W4 decreased slightly as the Re increased below 80,000. Yet these changes in $C_{L}$ for W4 were within the uncertainty below 80,000 Re. However, the observed changes in $C_{L}$ were more noticeable than W2 or W3 when the Re was below 80,000. 


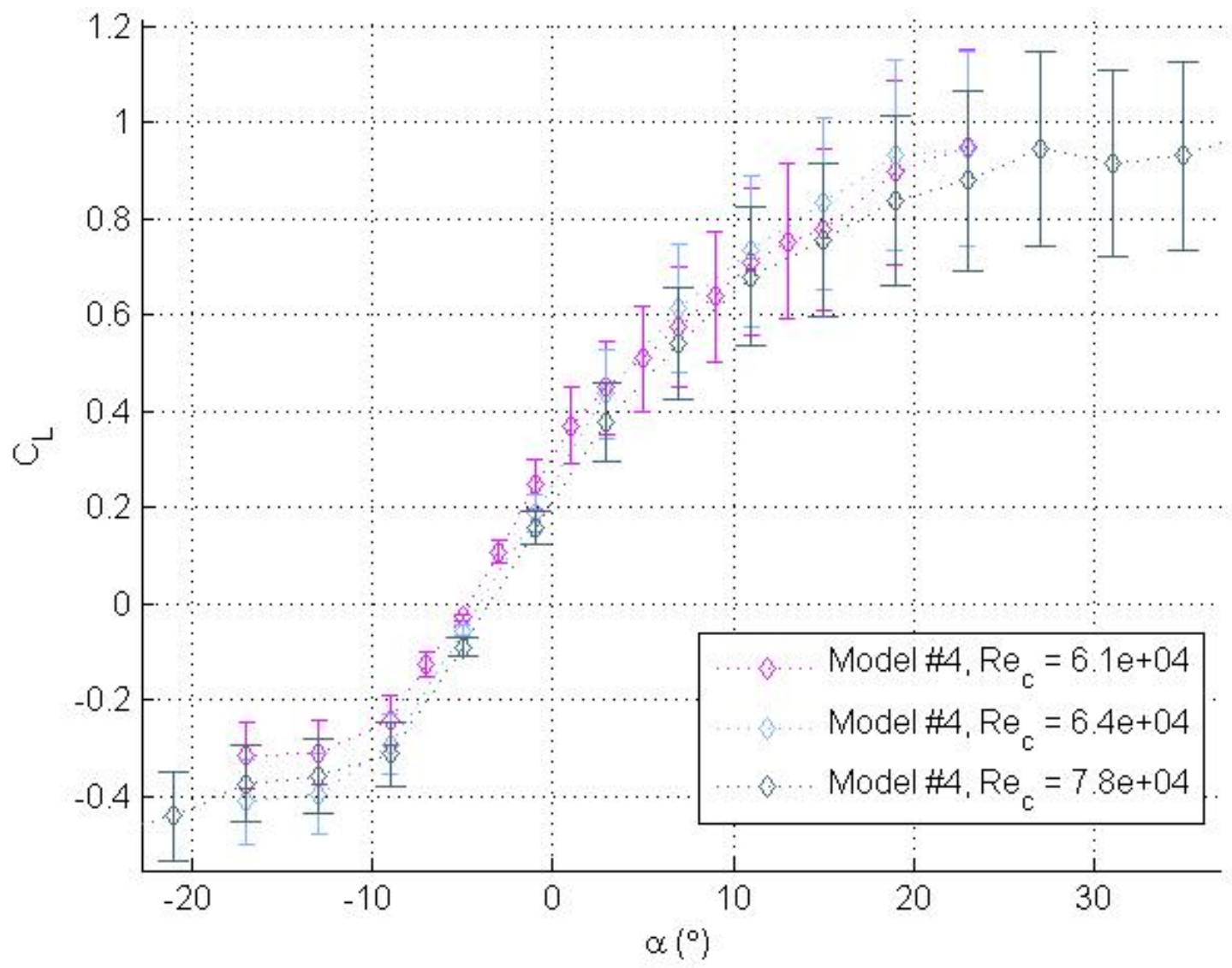

Figure 76: Cast Wing Model $C_{L}$ Sensitivity to Reynolds Number Below 80,000 Re

The $C_{L}$ of W4 became even more consistent when $R e$ was increased above 80,000. Figure 77 shows that no changes in $C_{L}$ occurred outside of the uncertainty under these conditions. 


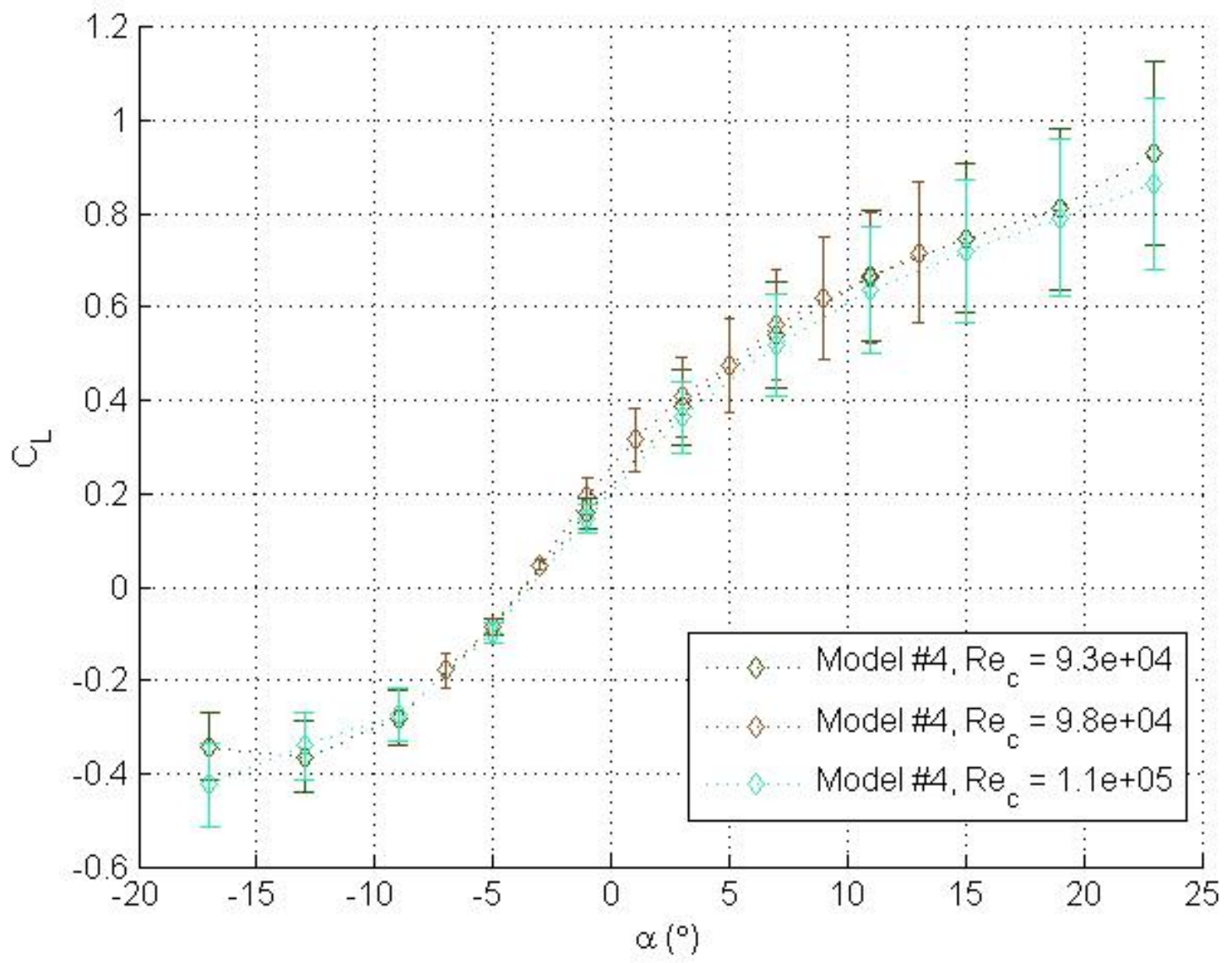

Figure 77: Cast Wing Model $C_{L}$ Sensitivity to Reynolds Number Above 80,000 Re

$R e$ was shown to have some effect on $C_{D}$ in the lower $R e$ range from 61,000 to 78,000 . An increase in $C_{D}$ outside of the uncertainty was observed between $-8^{\circ}$ and $-2^{\circ} \alpha$. This can be seen in Figure 78. 


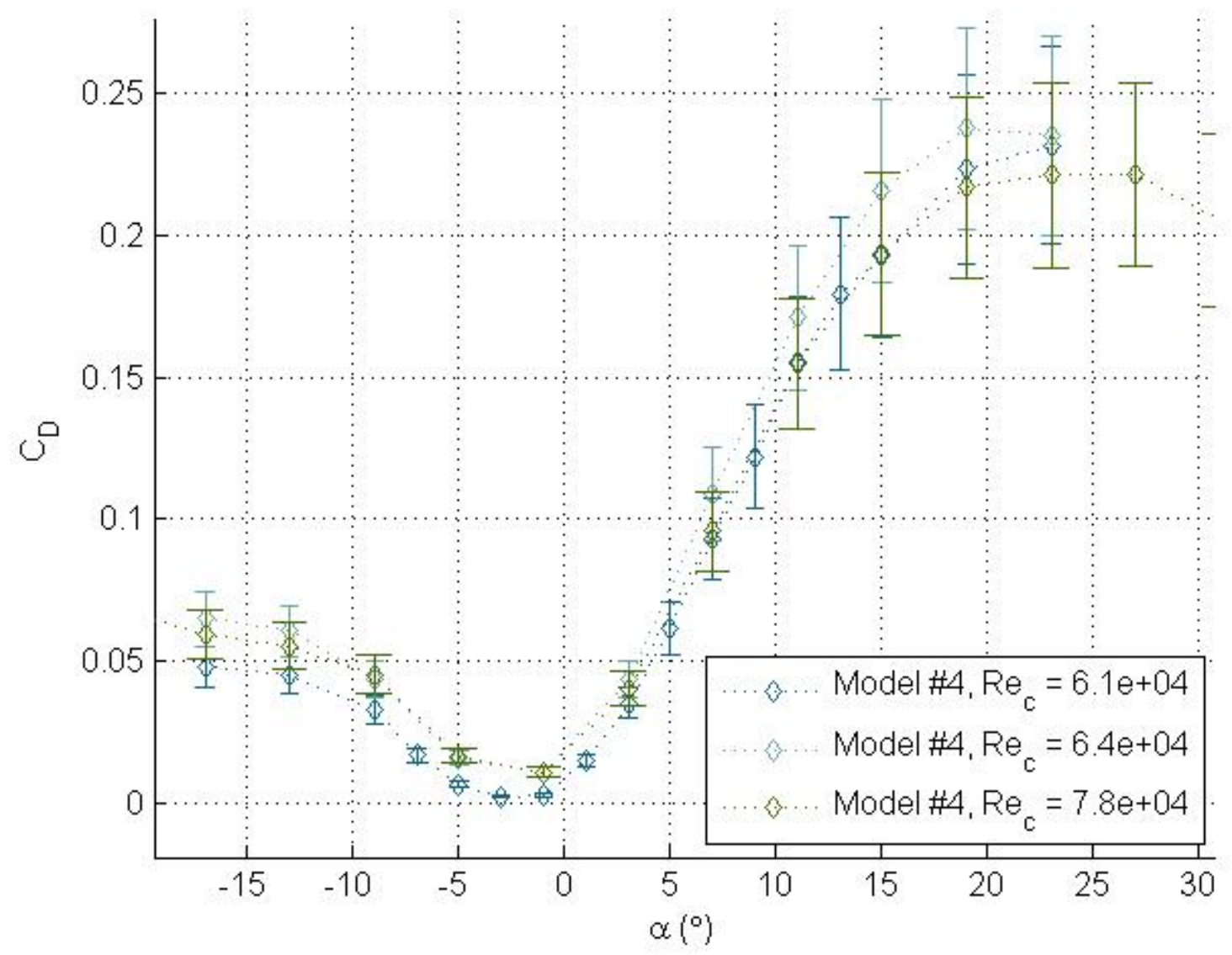

Figure 78: Cast Wing Model $C_{D}$ Sensitivity to Reynolds Number Below 80,000 Re

Figure 79 shows that the same consistency in $C_{D}$ continued into the higher $R e$ range.

Above 80,000 Re, the $C_{D}$ curves for $\mathrm{W} 4$ were almost the same. No differences resulting from changes in $R e$ above 80,000 occurred outside of the uncertainty. 


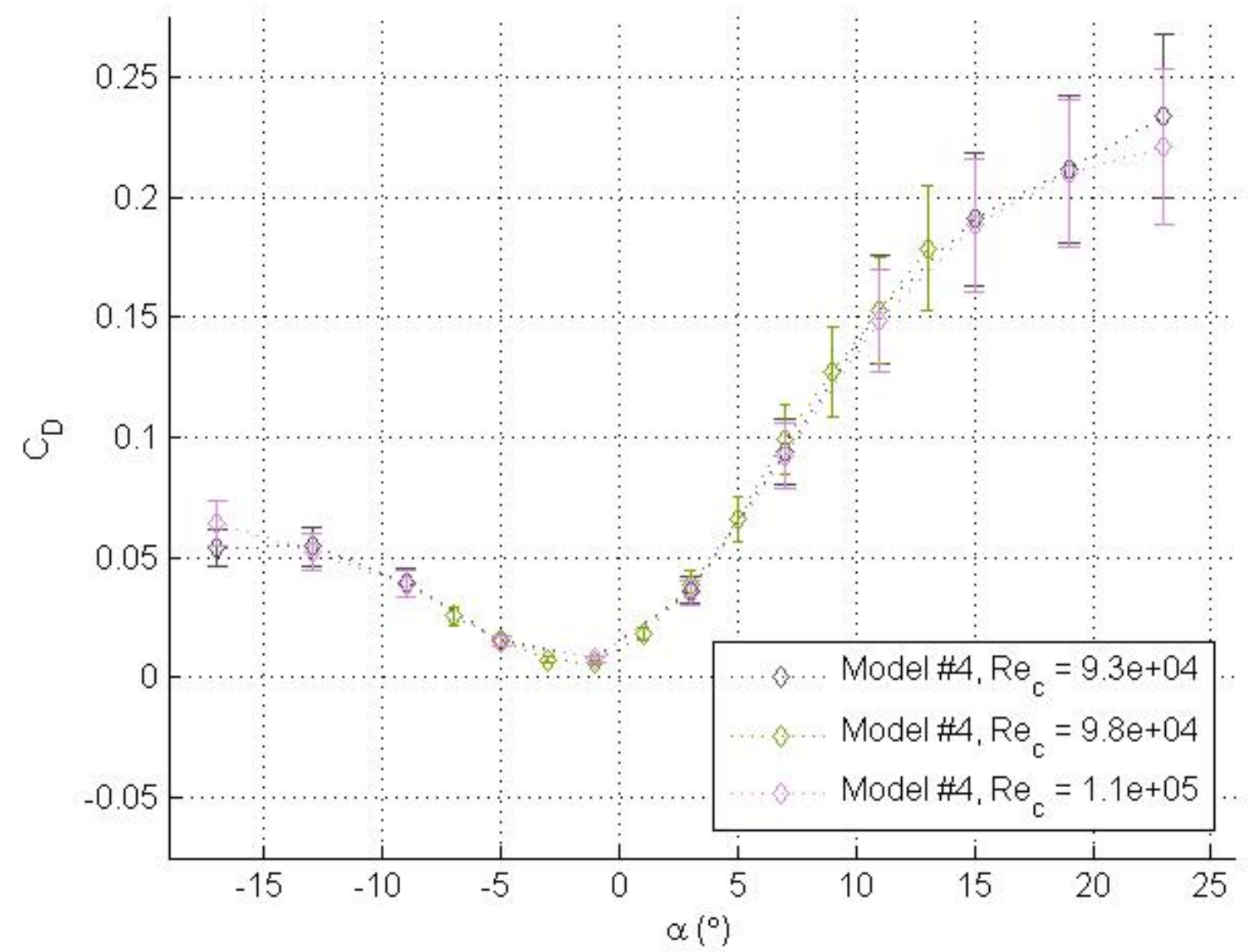

Figure 79: Cast Wing Model $C_{D}$ Sensitivity to Reynolds Number Above 80,000 Re

W5 was affected more by the Re than W2, W3, and W4. One example of this can be seen in Figure 80. $C_{L}$ increased between $-6^{\circ}$ and $-2^{\circ} \alpha$ as the $R e$ was increased to 78,000. 


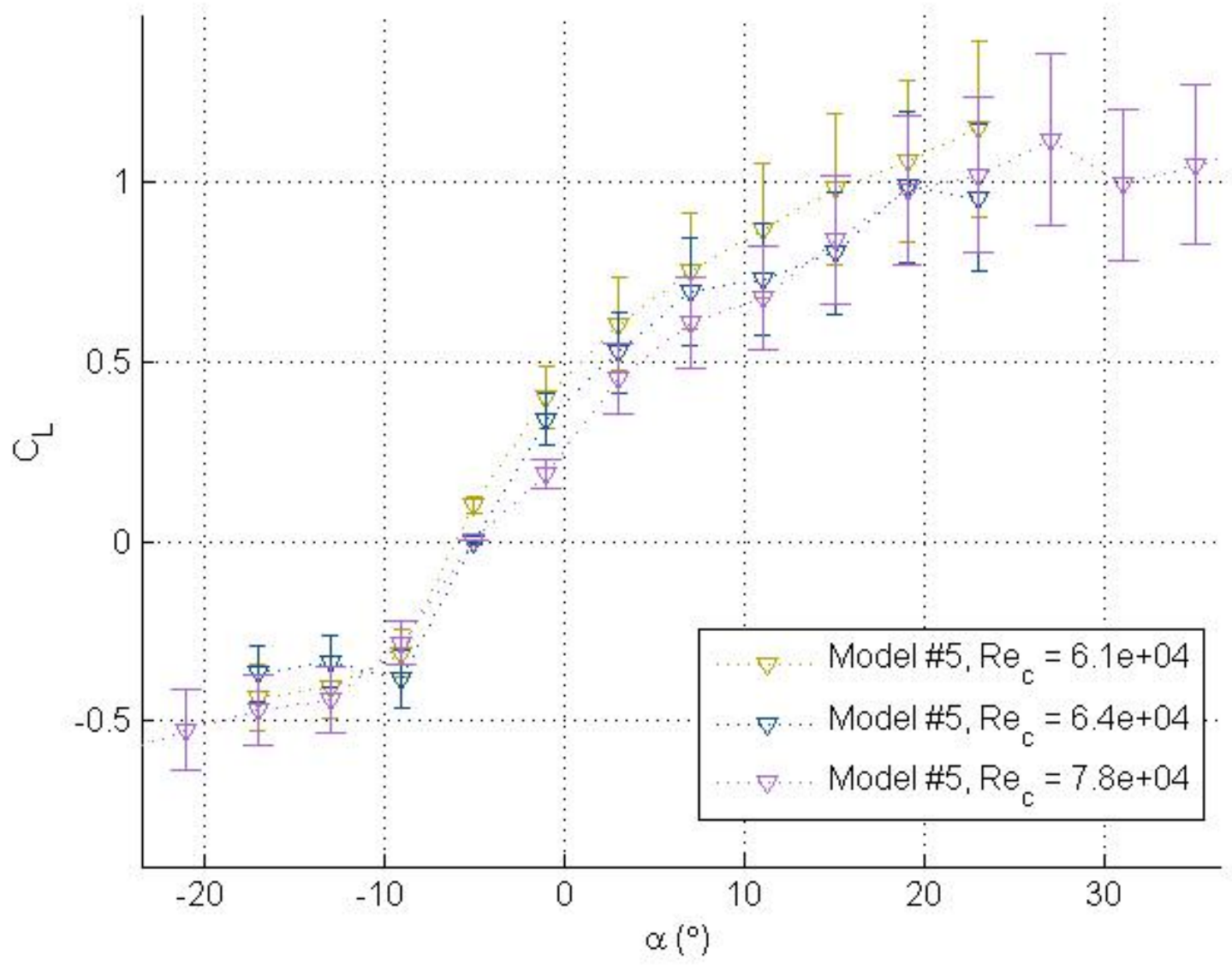

Figure 80: Effects of the Reynolds Number on $C_{L}$ of the Plasti Dipped Wing Model Below 80,000 Re

An increase in $C_{L}$ was also observed over the same $\alpha$ range when the $R e$ was increased from 93,000 to 104,000. $C_{L}$ then reduced when the $R e$ was increased to 109,000 . However, Figure 81 shows that most of these changes were within the uncertainty. 


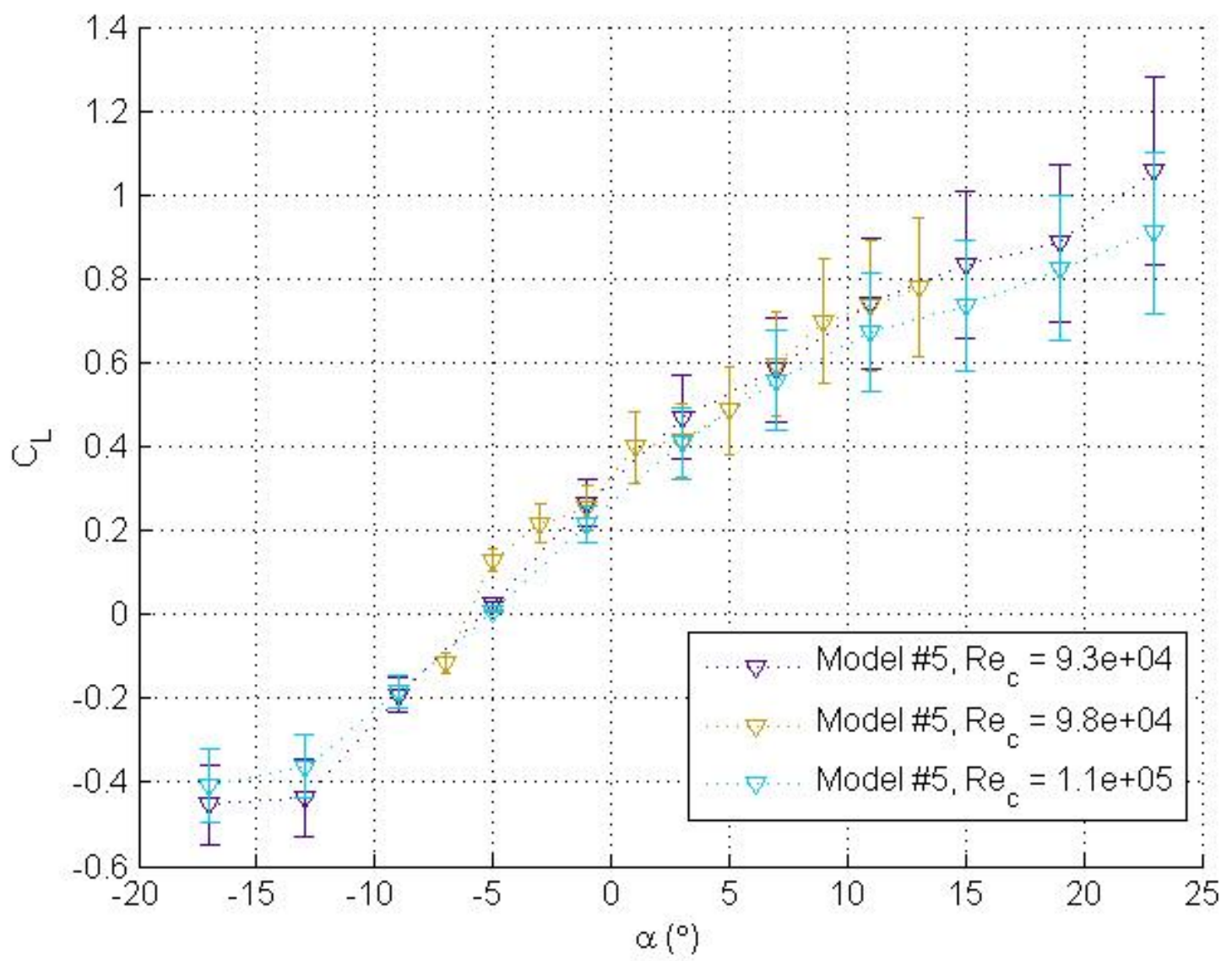

Figure 81: Effects of the Reynolds Number on $C_{L}$ of the Plasti Dipped Wing Model Above 80,000 Re

$C_{D}$ of W5 was affected by the Re similar to W4. Figure 82 shows that as the $R e$ was increased from 61,000 to $78,000, C_{D}$ increased between $-8^{\circ}$ and $-6^{\circ} \alpha$. All other changes were within the uncertainty. 


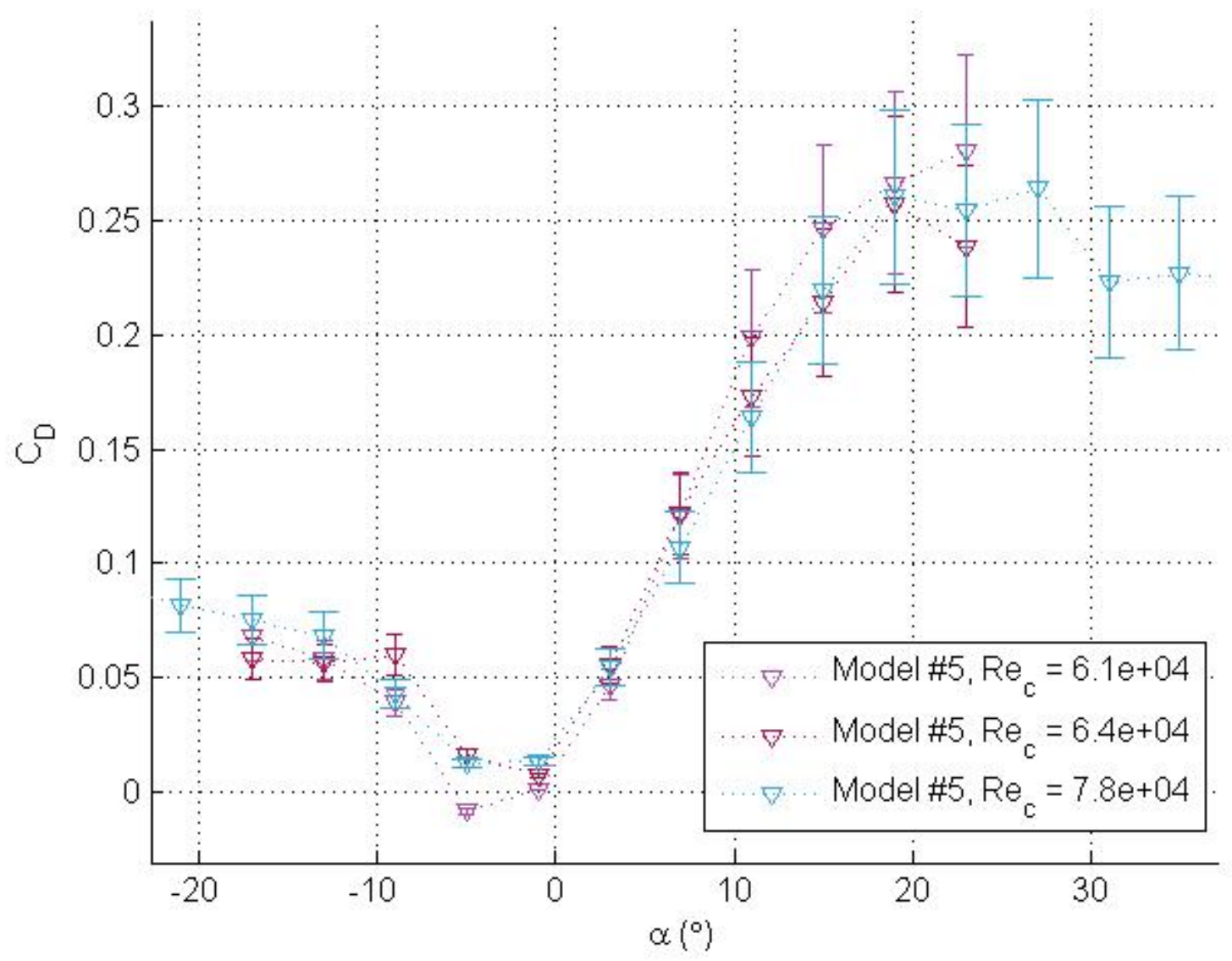

Figure 82: Effects of the Reynolds Number on $C_{D}$ of the Plasti Dipped Wing Model Below $80,000 \mathrm{Re}$

Unlike W4, a decrease in $C_{D}$ was seen between $-8^{\circ}$ and $2^{\circ} \alpha$ when the $\operatorname{Re}$ was 104,000 . This can be seen in Figure 83. An increase in $C_{L}$ also occurred under these conditions. This sudden increase in performance at 104,000 Re resembles the increased performance of W1 at 85,000 Re. 


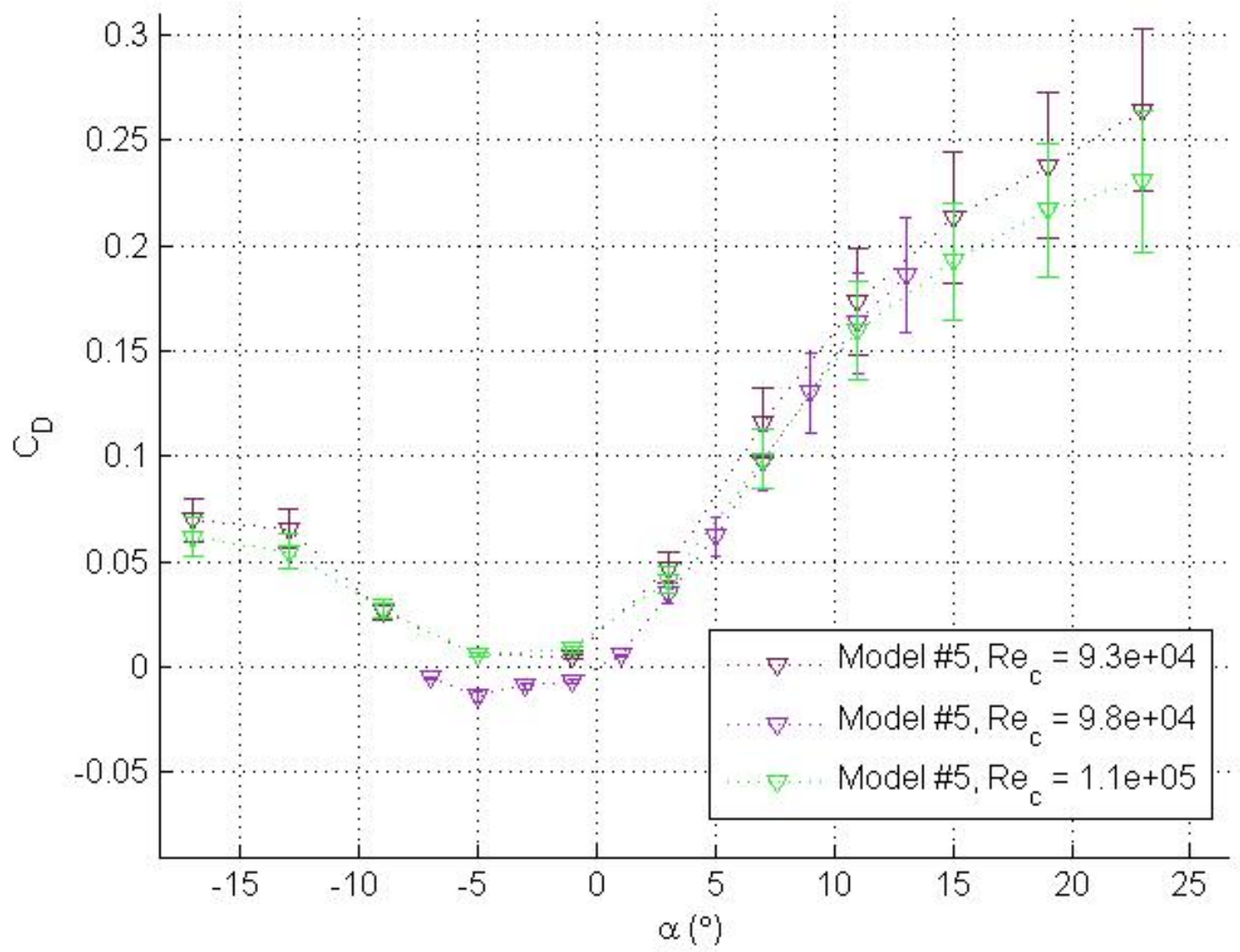

Figure 83: Effects of the Reynolds Number on $C_{D}$ of the Plasti Dipped Wing Model Above $80,000 R e$

\subsubsection{Impacts of Feather Motion}

To fully understand the impacts of feather motion, the data collected from W1, W2, W3, and W4 were compared. Each of these models exhibited a different level of feather motion. The feathers of W1 were not inhibited. Feather motion on W2 was altered moderately using hair spray. W3 feather motion was largely inhibited by the application of polyurethane. W4 was a cast of W2. Therefore, W4 provided an example of the same wing with no moving feathers. 
Preserved Wing Model vs. Hair Sprayed Wing Model

Figure 84 shows that W2 out performs W1 when the Re is below 80,000. W2 also reaches its peak performance at 78,000 Re as compared to W1 at 85,000 Re. The increased $C_{L}$ and $C_{D}$ of W2 occur above $\alpha=-8^{\circ}$ when $R e=63,000$. However, the only certain increases in these values occur between $6^{\circ}$ and $20^{\circ}$ angle of attack when $R e=78,000$. 


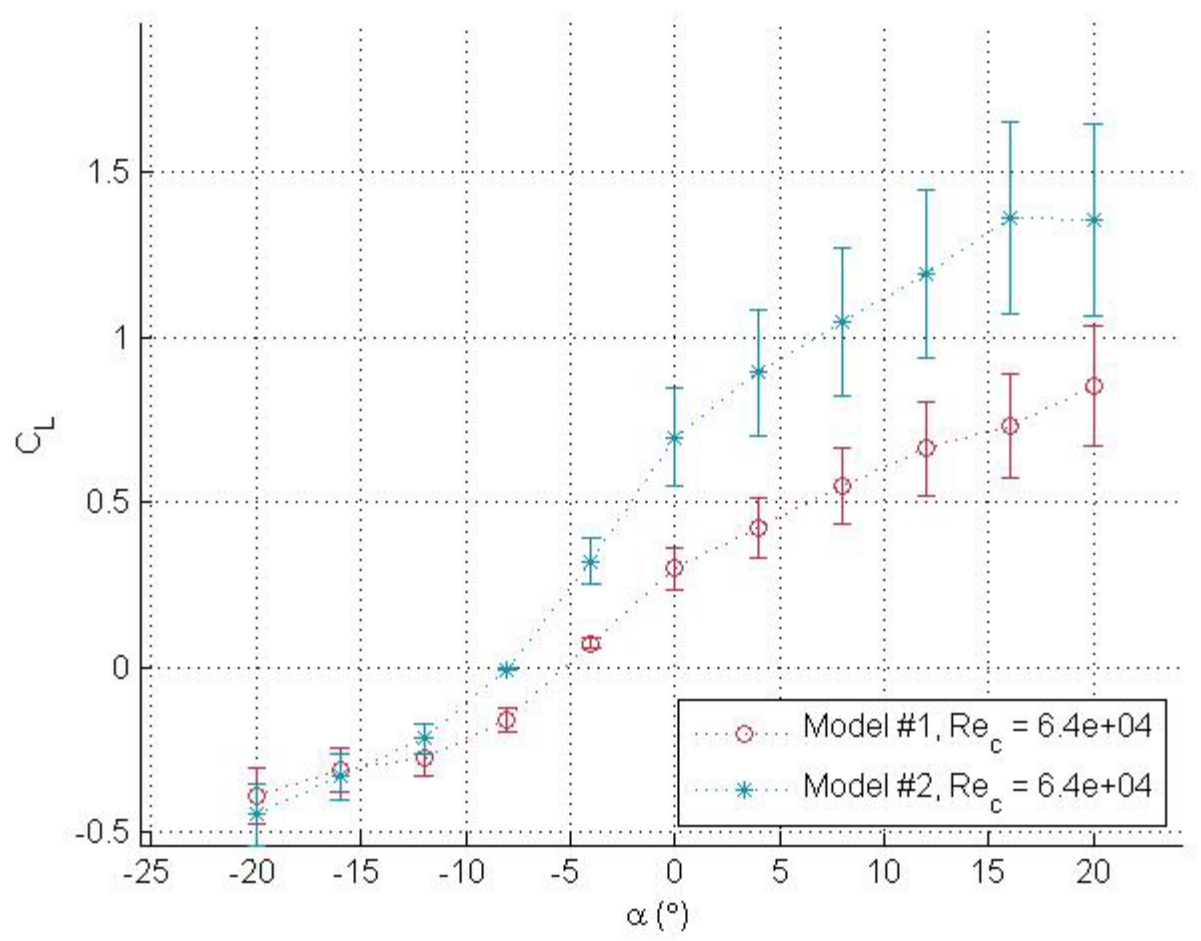

Figure 84: Comparison of the Differences in $C_{L}$ between the Preserved and Hair Sprayed Wing Models at 64,000 Re

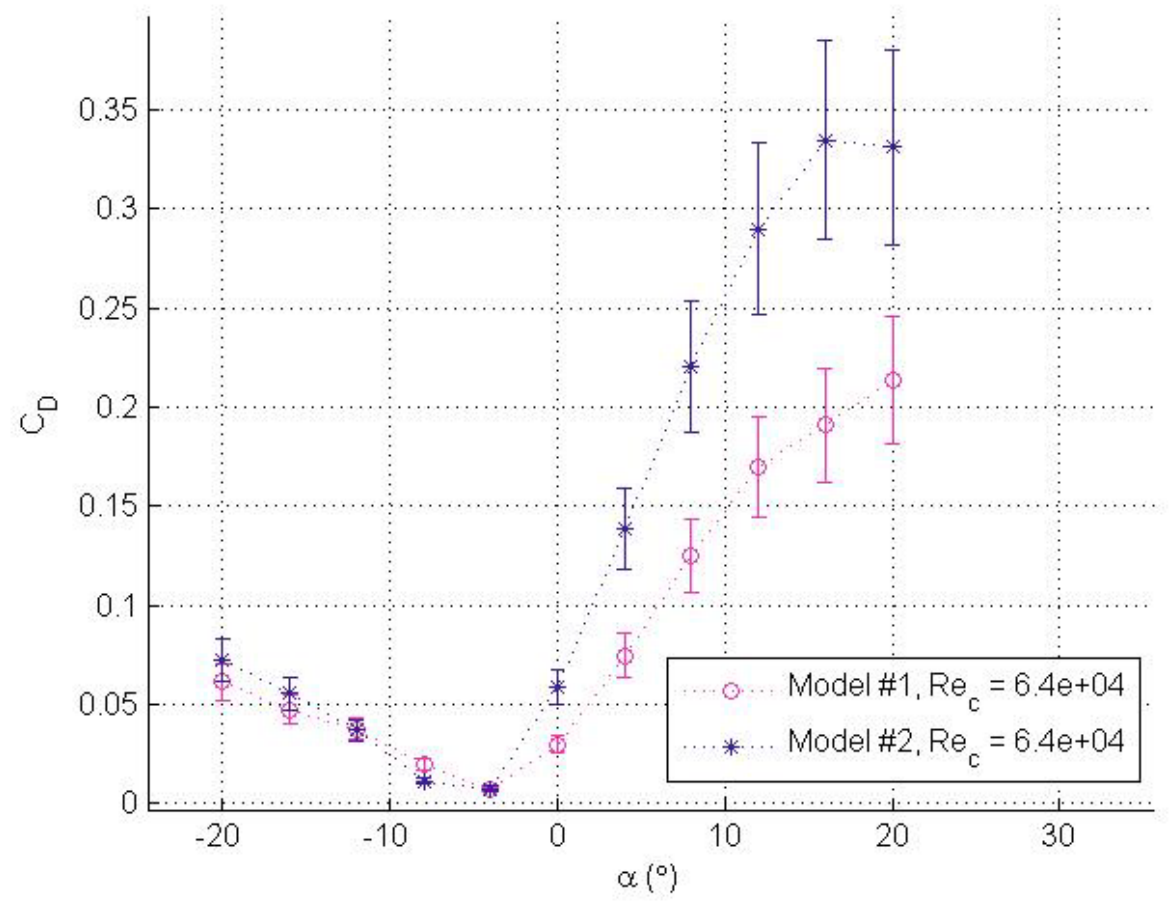

Figure 85: Comparison of the Differences in $C_{D}$ between the Preserved and Hair Sprayed Wing Models at 64,000 Re 


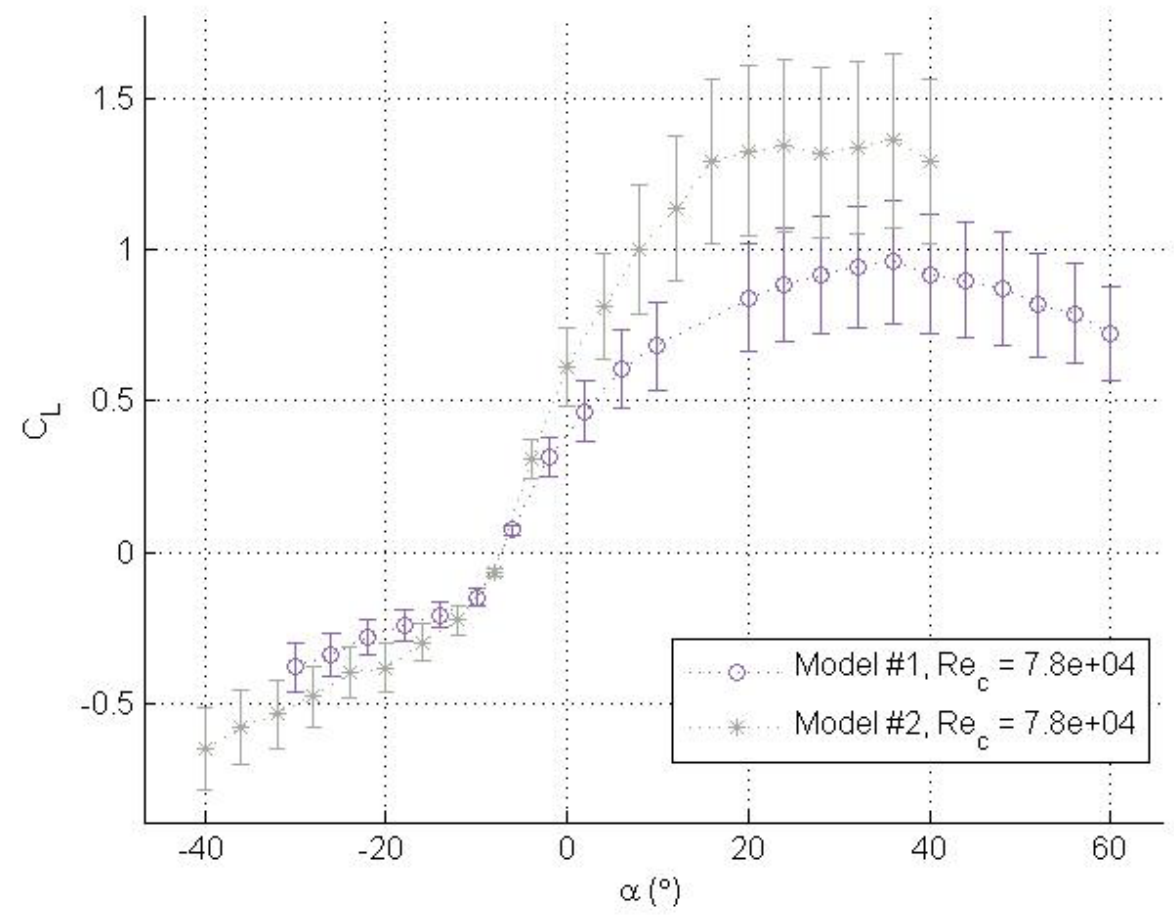

Figure 86: Comparison of the Differences in $C_{L}$ between the Preserved and Hair Sprayed Wing Models at 78,000 Re

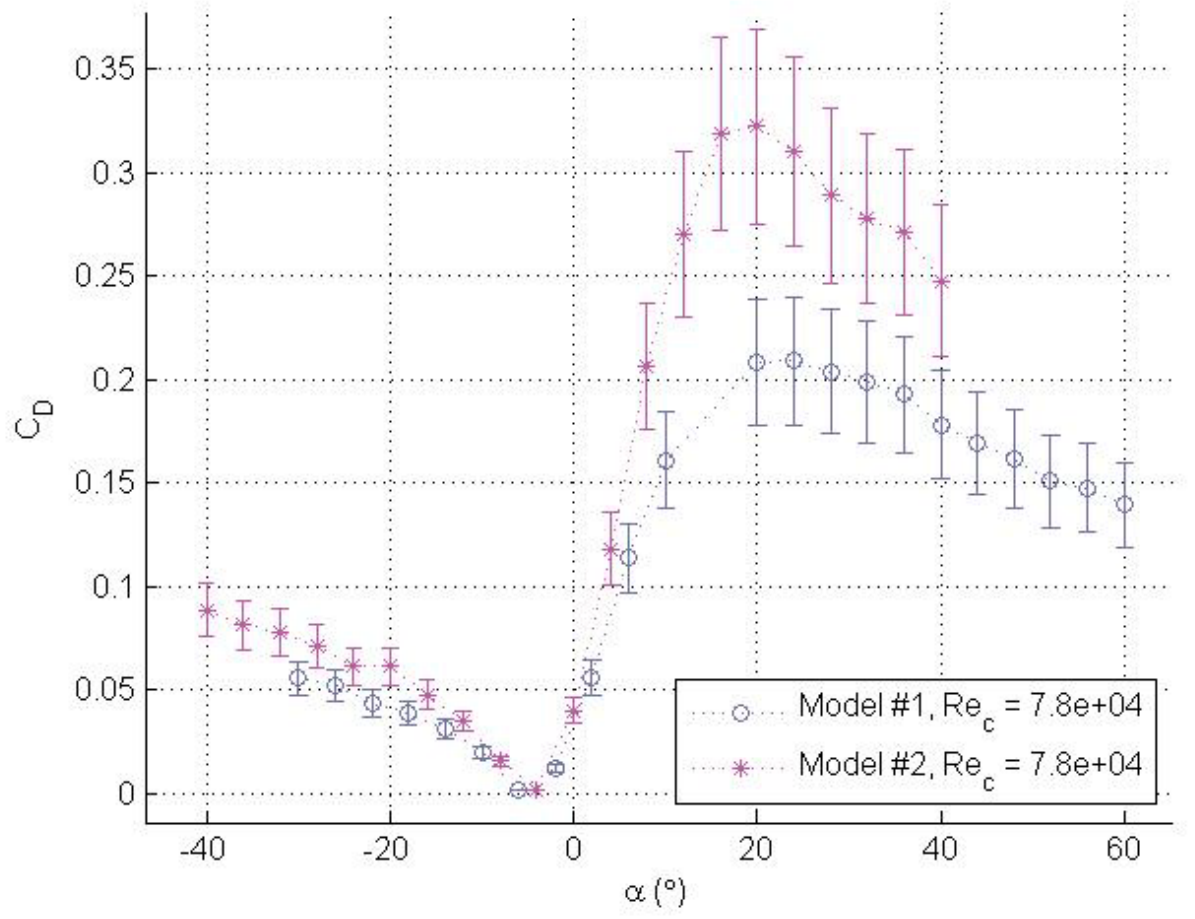

Figure 87: Comparison of the Differences in $C_{D}$ between the Preserved and Hair Sprayed Wing Models at 78,000 Re 


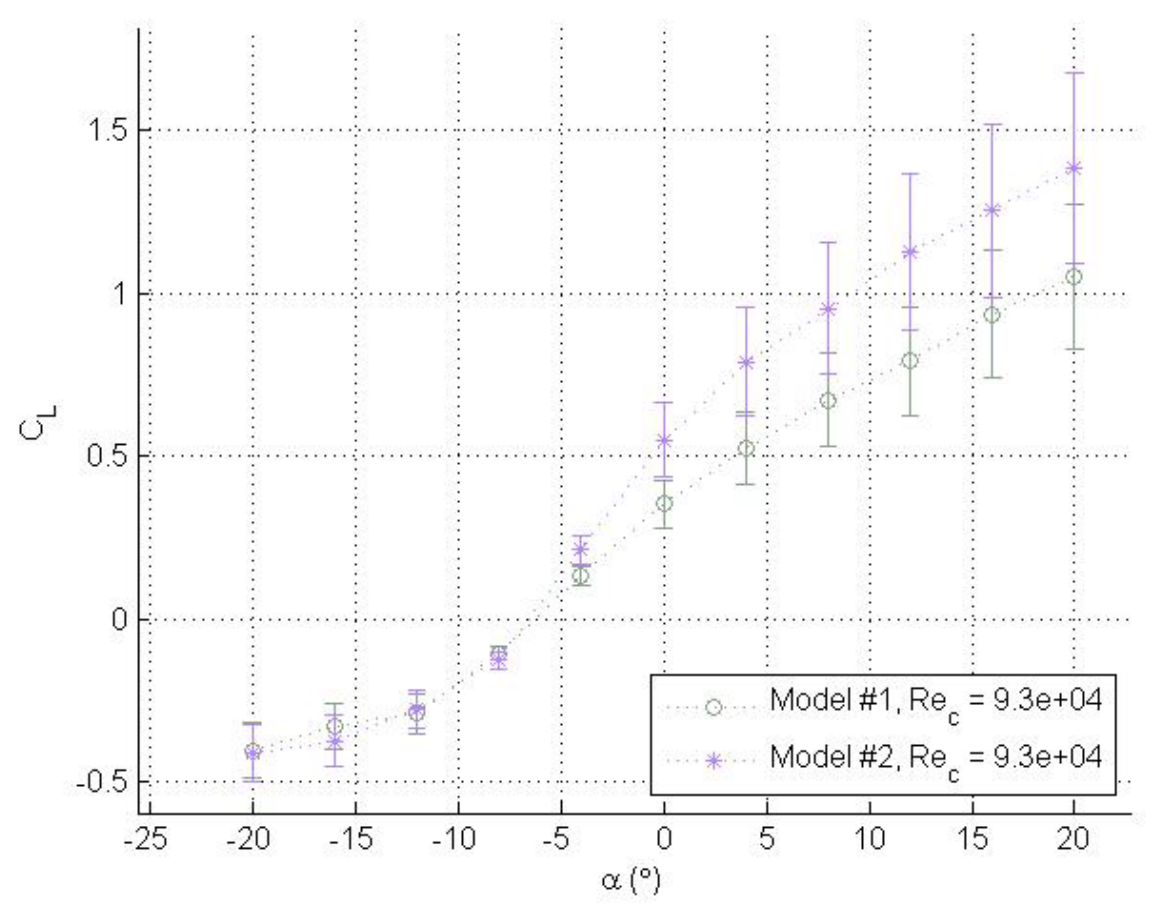

Figure 88: Comparison of the Differences in $C_{L}$ between the Preserved and Hair Sprayed Wing Models at 93,000 Re

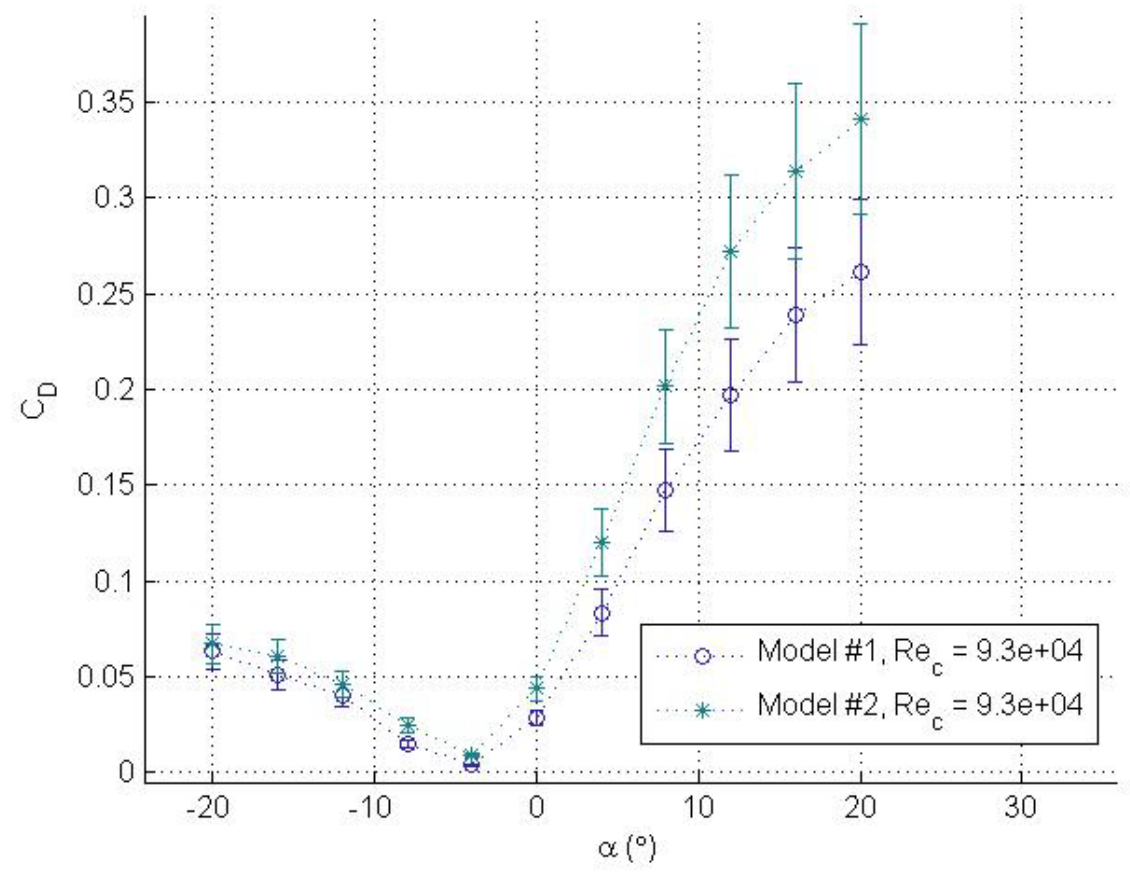

Figure 89: Comparison of the Differences in $C_{D}$ between the Preserved and Hair Sprayed Wing Models at 93,000 Re 


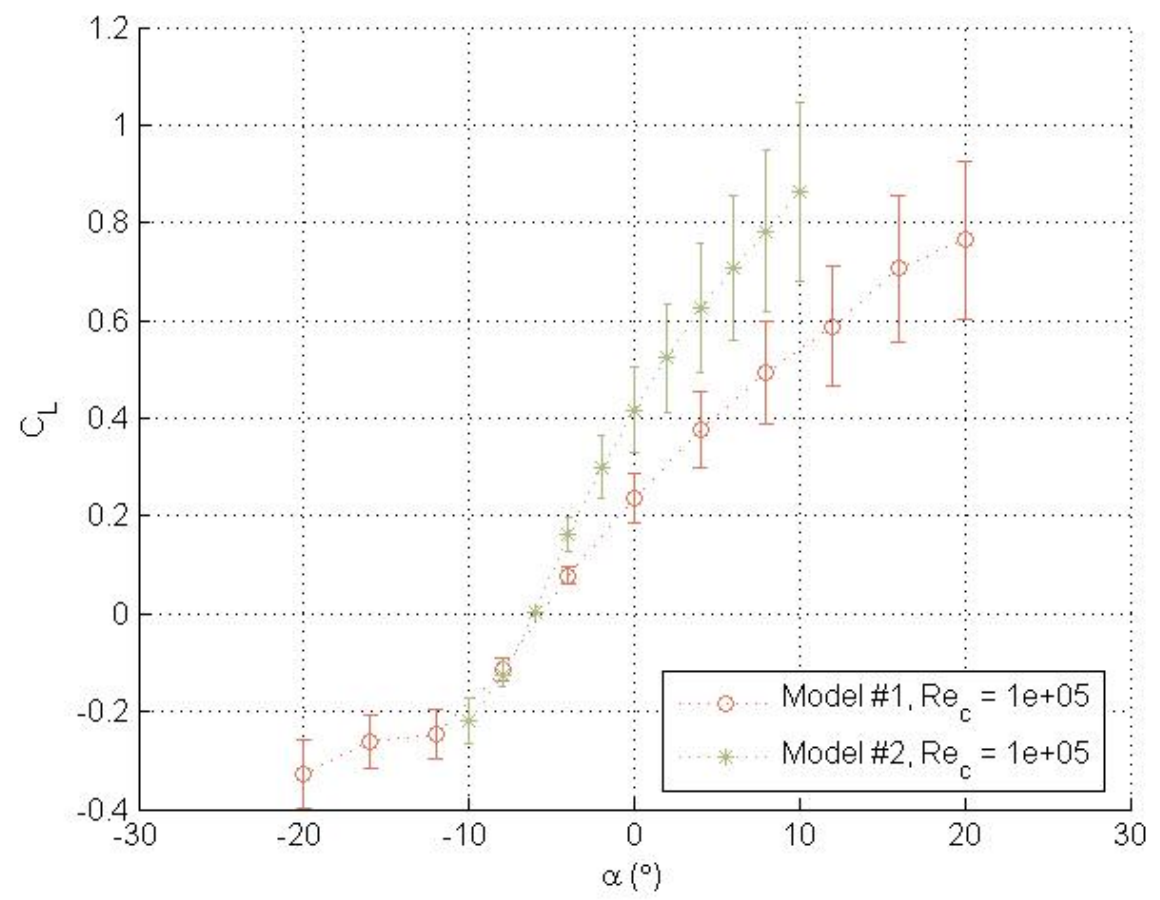

Figure 90: Comparison of the Differences in $C_{L}$ between the Preserved and Hair Sprayed Wing Models at 100,000 Re

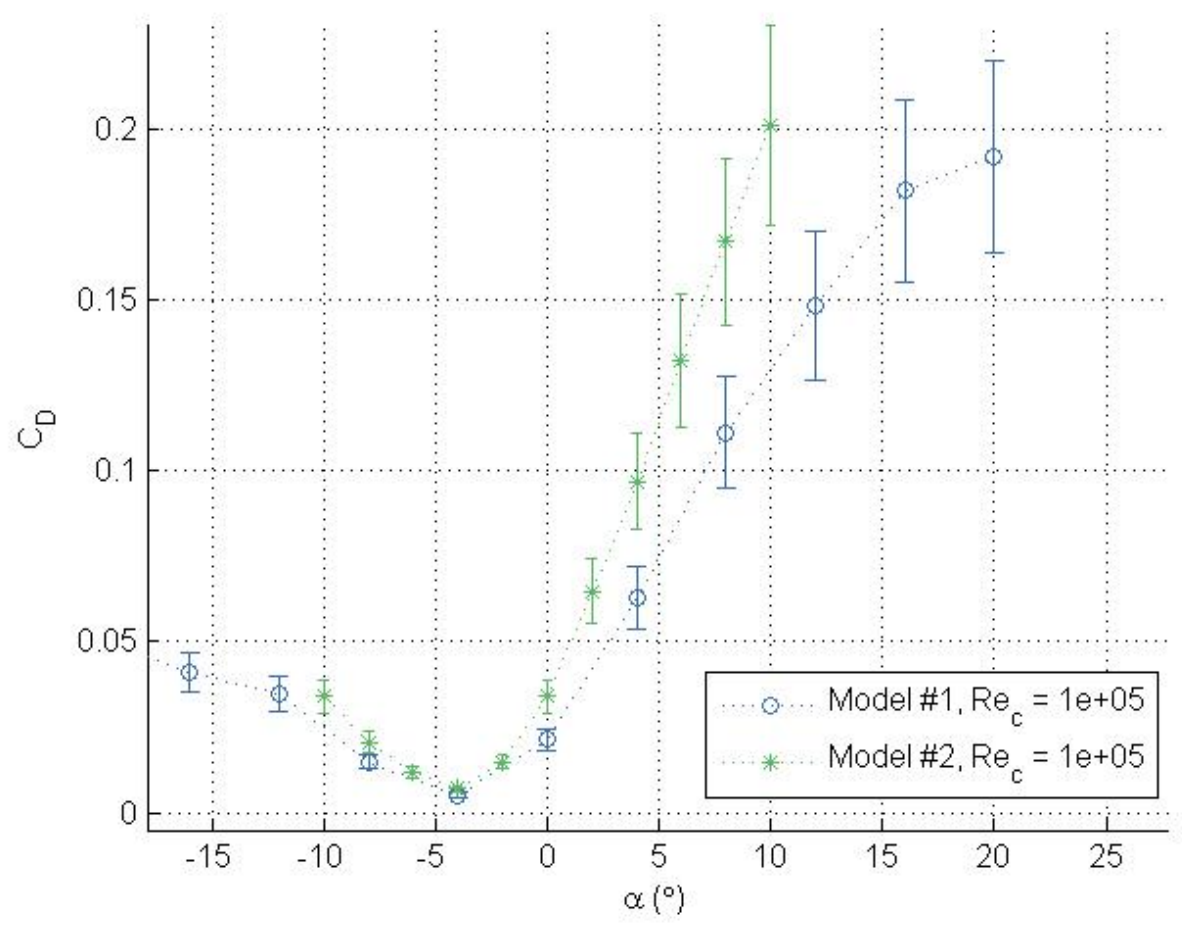

Figure 91: Comparison of the Differences in $C_{D}$ between the Preserved and Hair Sprayed Wing Models at 100,000 Re 


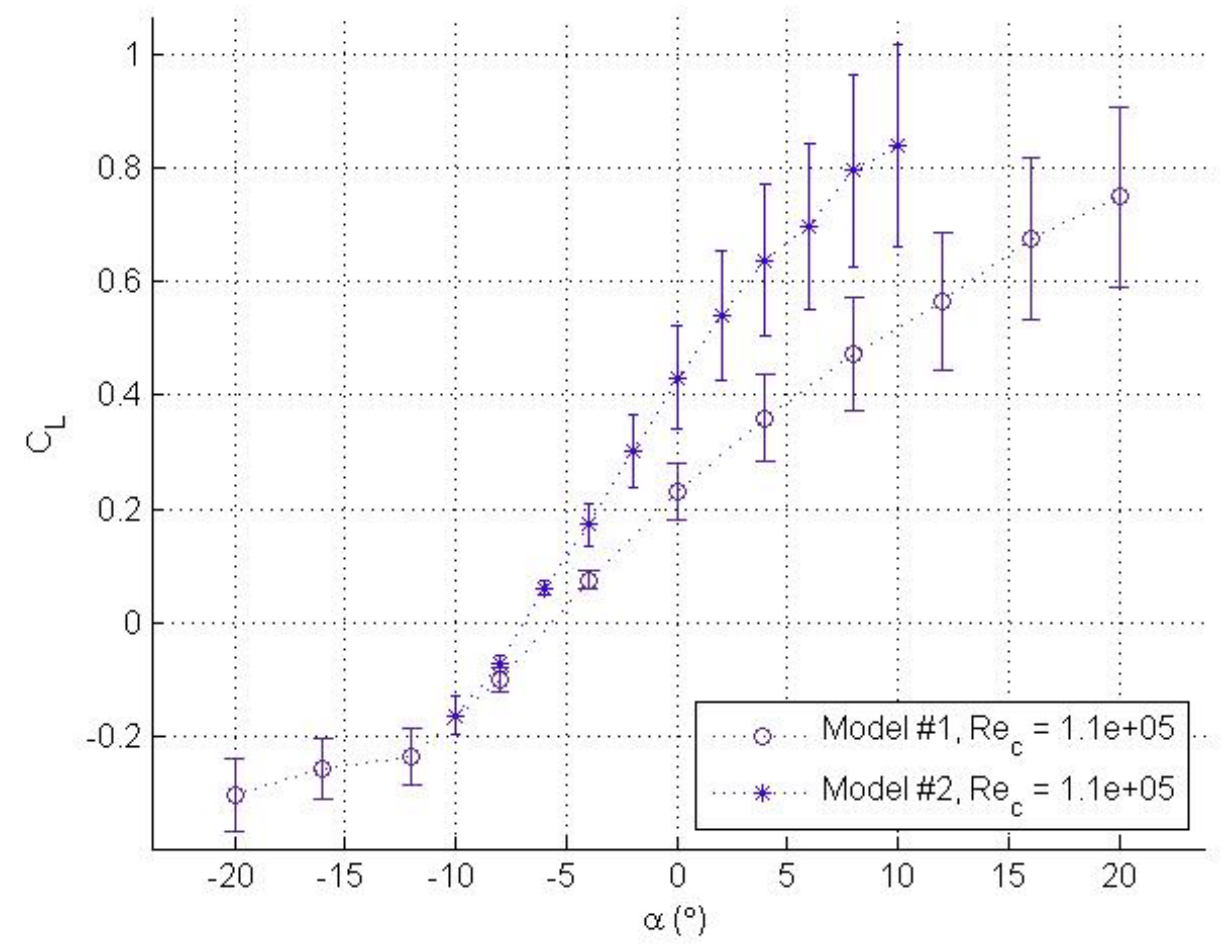

Figure 92: Comparison of the Differences in $C_{L}$ between the Preserved and Hair Sprayed Wing Models at 110,000 Re

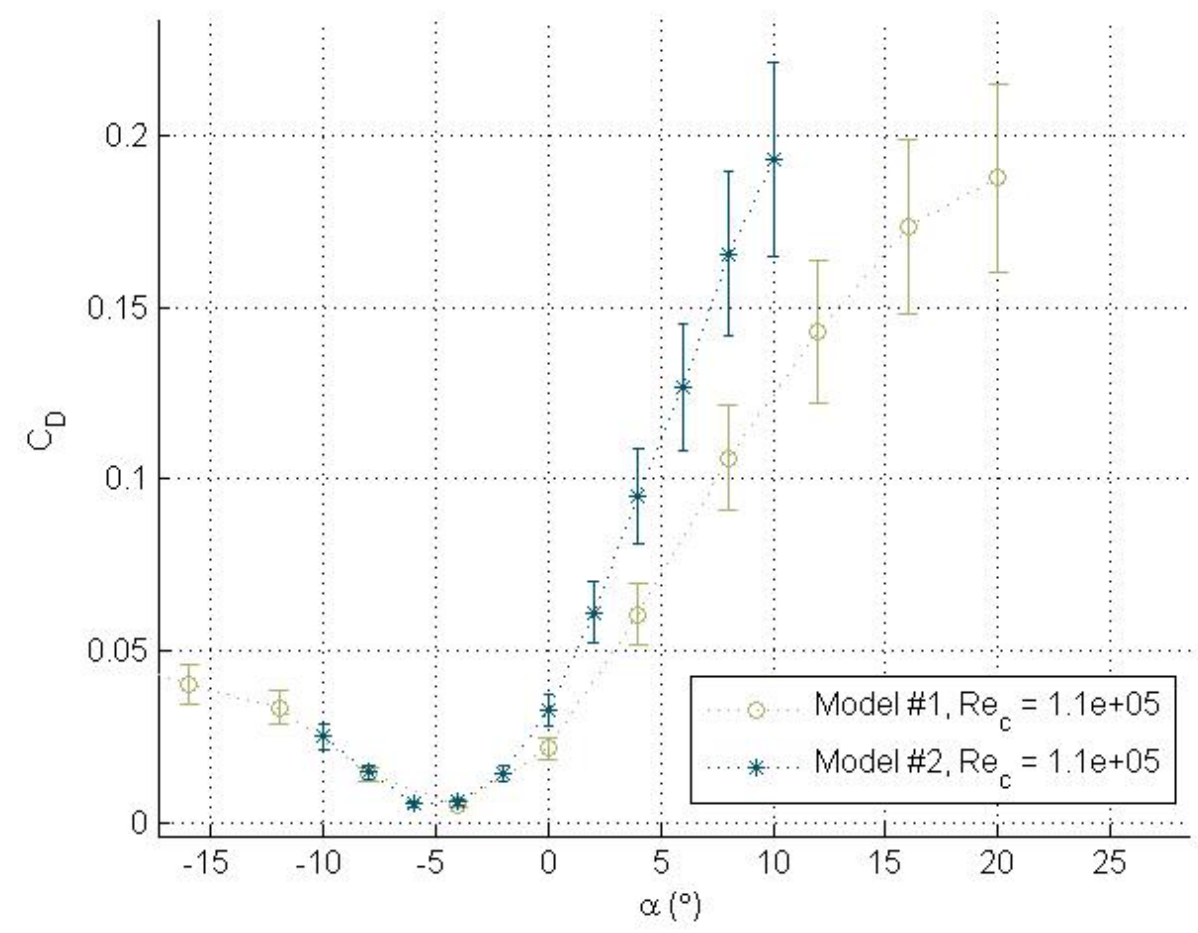

Figure 93: Comparison of the Differences in $C_{D}$ between the Preserved and Hair Sprayed Wing Models at 110,000 Re 
As the Re was increased from 80,000, the $C_{L}$ and $C_{D}$ curves of both wings decreased. This decrease occurred at different rates for each model. When compared to W1, W2 exhibited increased $C_{L}$ values between $-5^{\circ}$ and $10^{\circ}$ angle of attack for this Re range. W2 also showed higher $C_{D}$ values than $\mathrm{W} 1$, but these values were only certain when the angle of attack was between $0^{\circ}$ and $10^{\circ}$.

W2 exhibited higher values of $C_{L}$ when the angle of attack was within $-5^{\circ}$ and $20^{\circ}$. When compared to W1, W2 also showed increases in $C_{D}$ between $-5^{\circ}$ and $10^{\circ}$ angle of attack. This corresponds with the increased frequency of covert and secondary covert feathers observed on W2. When compared to the frequency of secondary covert feathers on W1, W2 showed a $40 \%$ increase when $\alpha=0^{\circ}$, but the coverts were inhibited at this position.

W2 covert feathers showed a frequency increase of $10 \%$ when $\alpha=40^{\circ}$ when compared to W1. However, the secondary coverts were immobilized at this angle of attack. When $\alpha=-40^{\circ}$, coverts were immobilized on both W1 and W2, but a 21\% increase was observed in the secondary covert frequency at this angle of attack on W2. A slight increase in the $C_{L}$ and $C_{D}$ was noticed at $\alpha=-40^{\circ}$, but this increase was still within the uncertainty.

\section{Preserved Wing Model vs. Polyurethane Coated Wing Model}

When $R e$ was below 80,000, W3 showed decreased $C_{L}$ values when compared to W1. This occurred when the angle of attack was below $-4^{\circ}$. Higher $C_{L}$ values for W1 were apparent for the rest of the plot. However, the only differences outside of the uncertainty were lower $C_{L}$ values for W3 when $\alpha=-4^{\circ}$. 


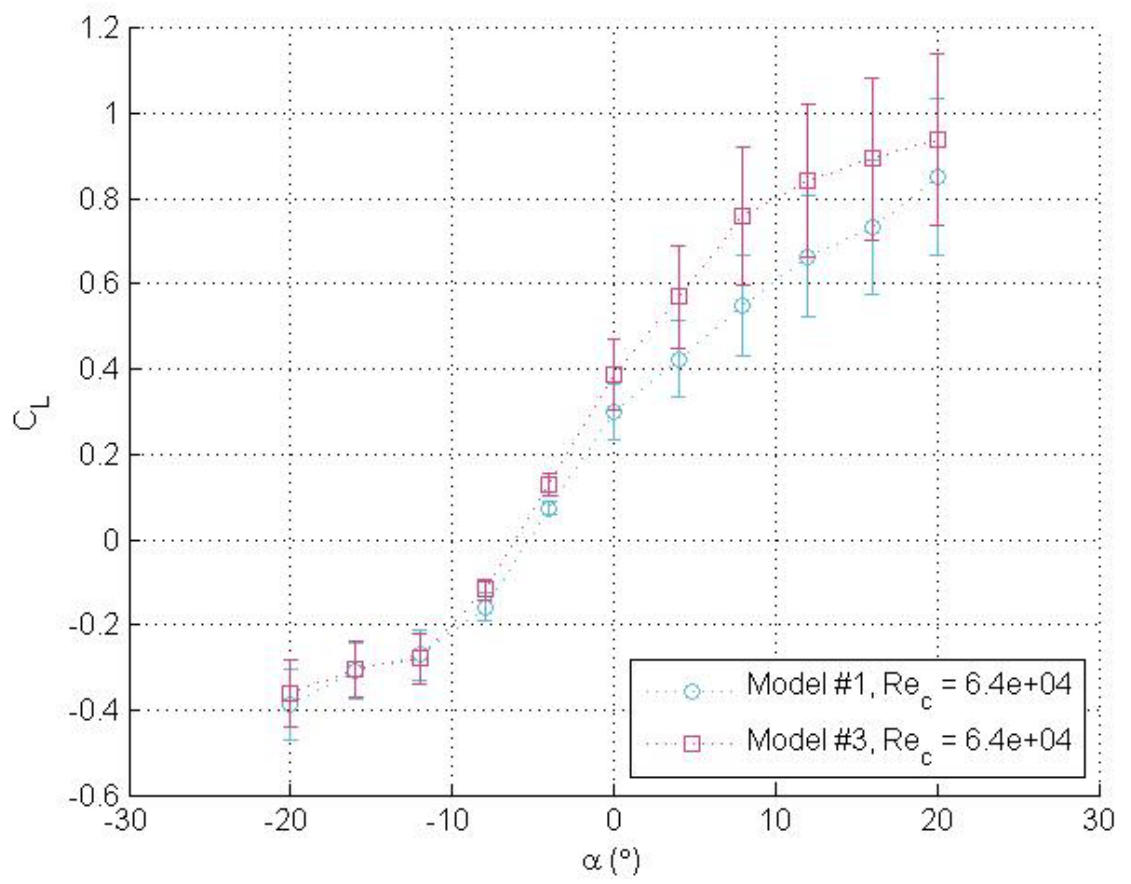

Figure 94: Comparison of the Differences in $C_{L}$ between the Preserved and Polyurethane Coated Wing Models at 64,000 Re

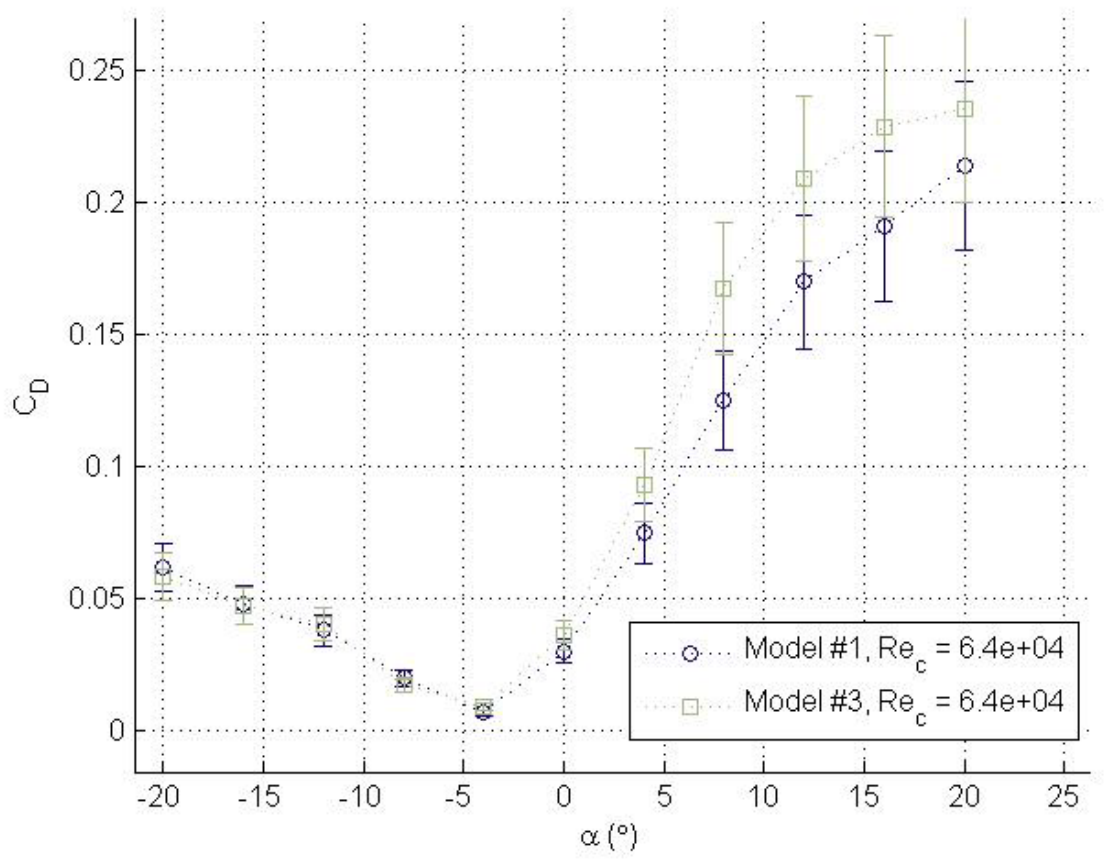

Figure 95: Comparison of the Differences in $C_{D}$ between the Preserved and Polyurethane Coated Wing Models at 64,000 Re 


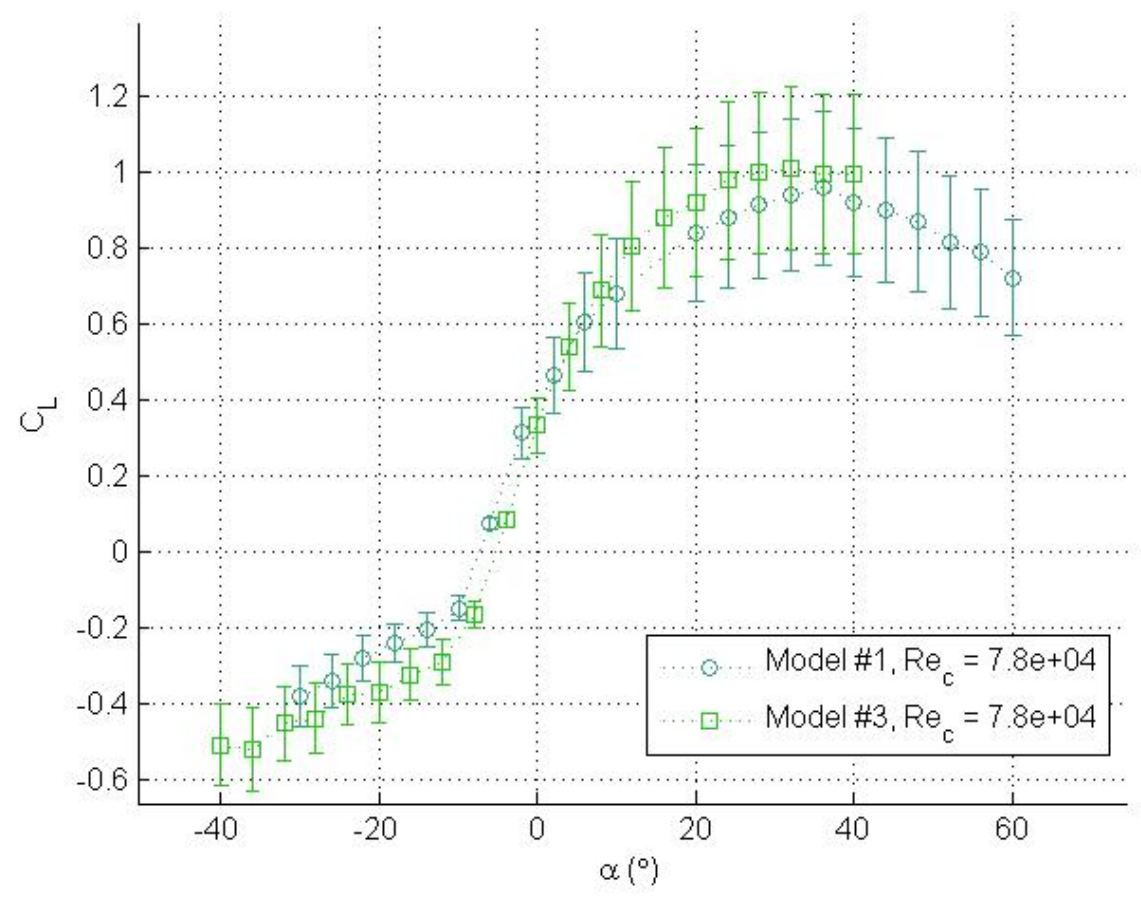

Figure 96: Comparison of the Differences in $C_{L}$ between the Preserved and Polyurethane Coated Wing Models at 78,000 Re

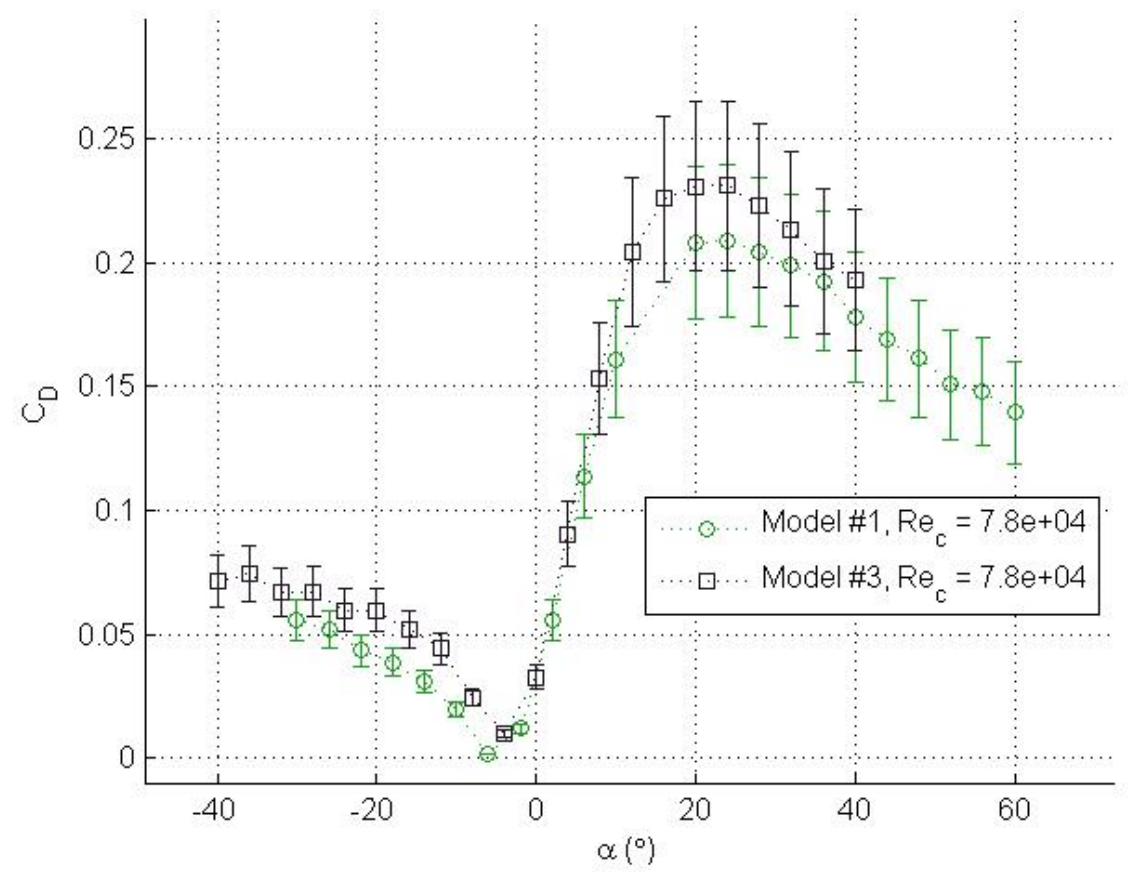

Figure 97: Comparison of the Differences in $C_{D}$ between the Preserved and Polyurethane Coated Wing Models at 78,000 Re 


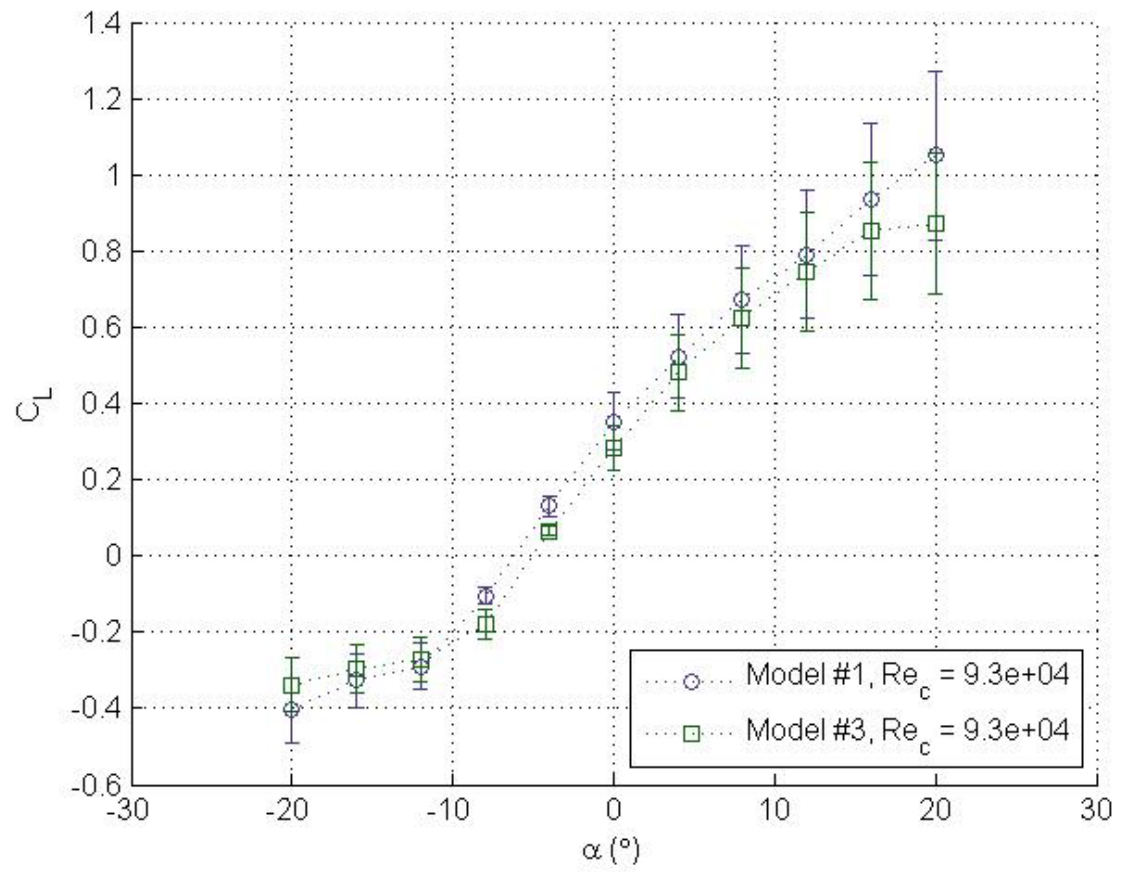

Figure 98: Comparison of the Differences in $C_{L}$ between the Preserved and Polyurethane Coated Wing Models at 93,000 Re

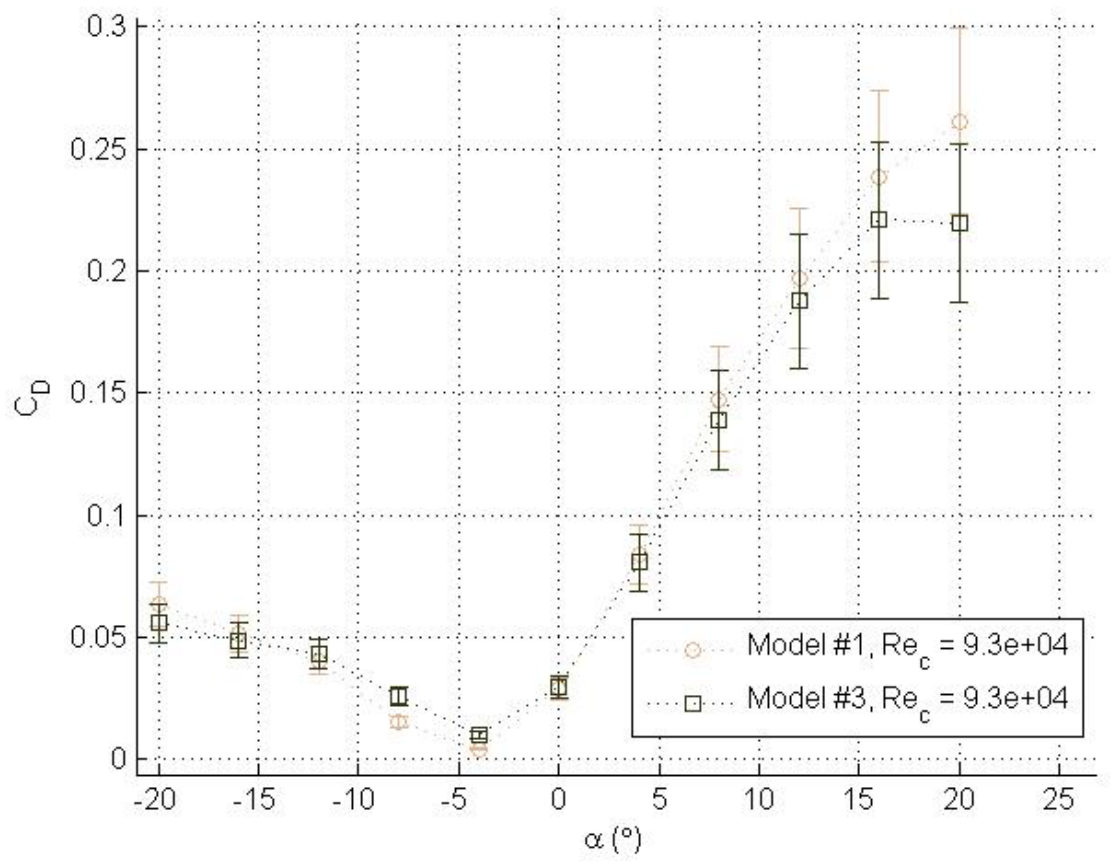

Figure 99: Comparison of the Differences in $C_{D}$ between the Preserved and Polyurethane Coated Wing Models at 93,000 Re 


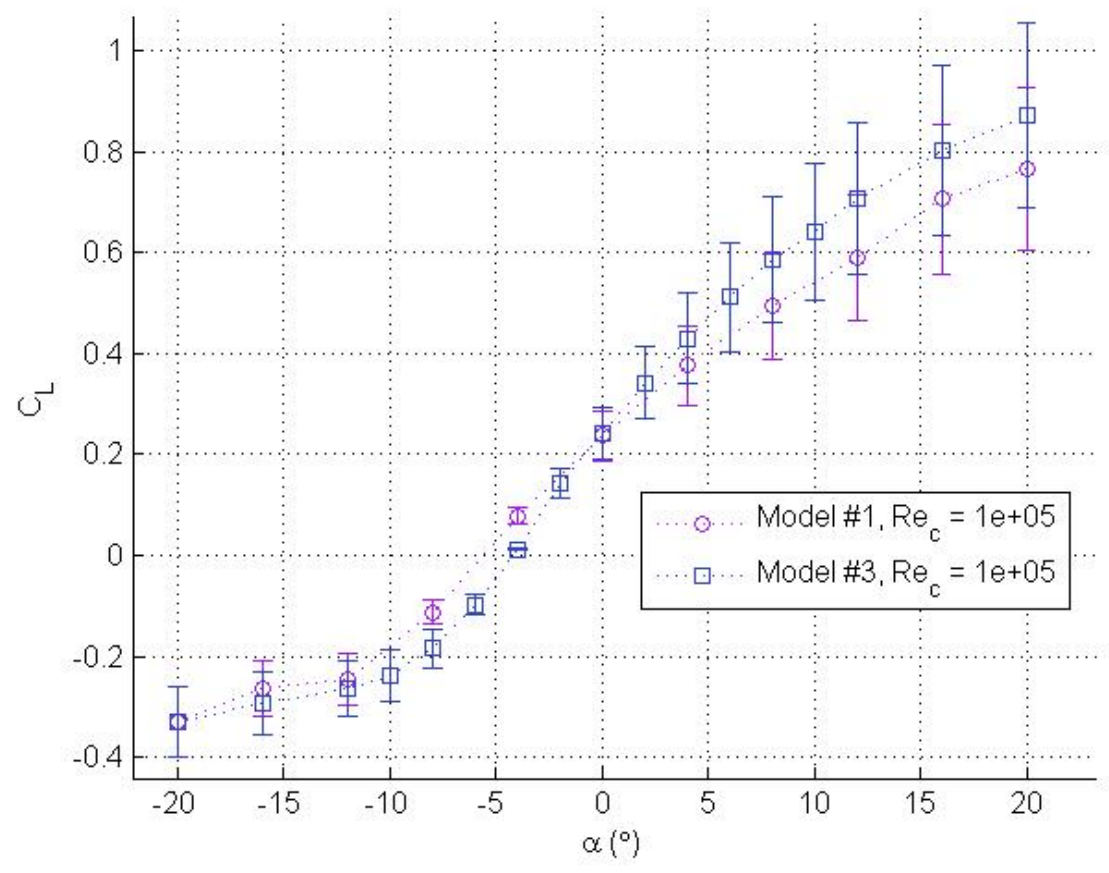

Figure 100: Comparison of the Differences in $C_{L}$ between the Preserved and Polyurethane Coated Models at 100,000 Re

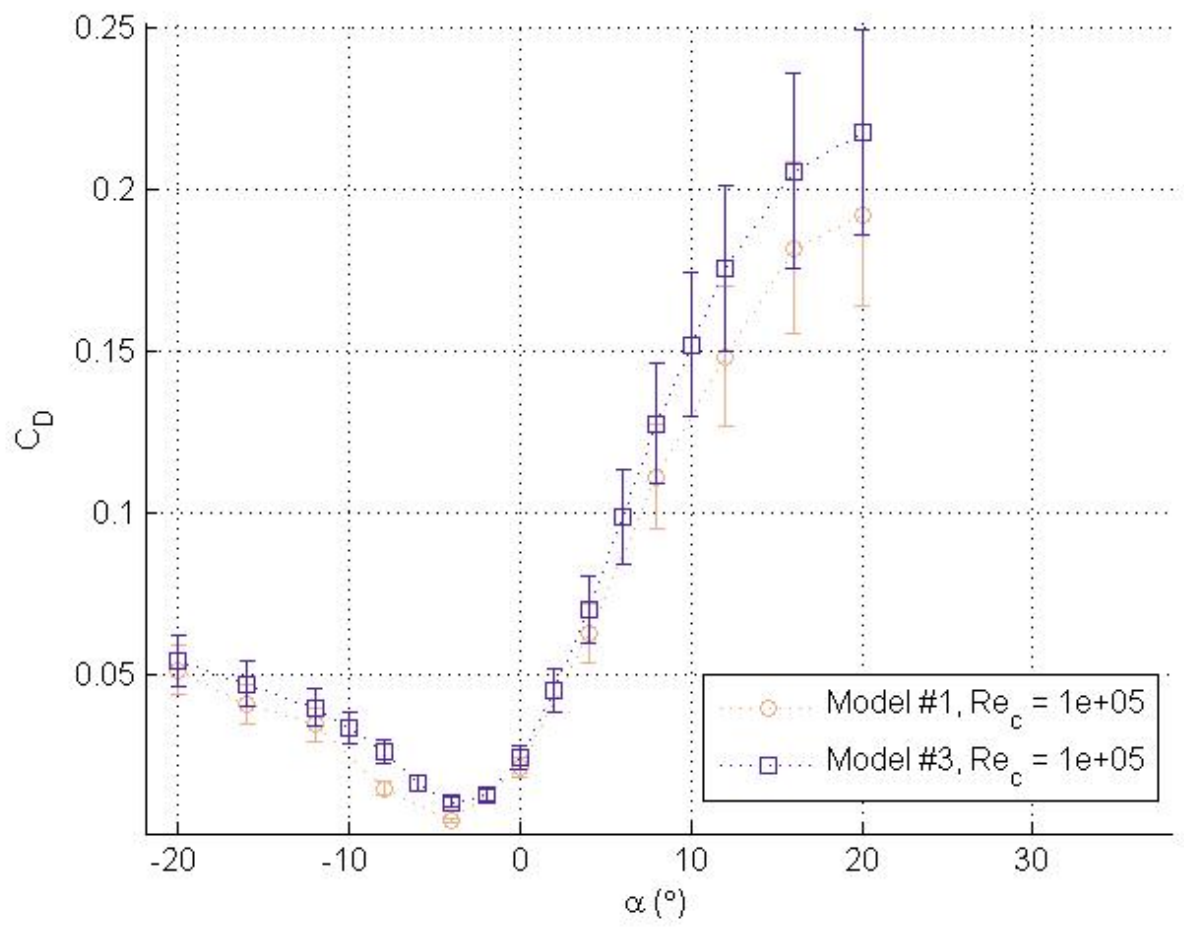

Figure 101: Comparison of the Differences in $C_{D}$ between the Preserved and Polyurethane Coated Models at 100,000 Re 


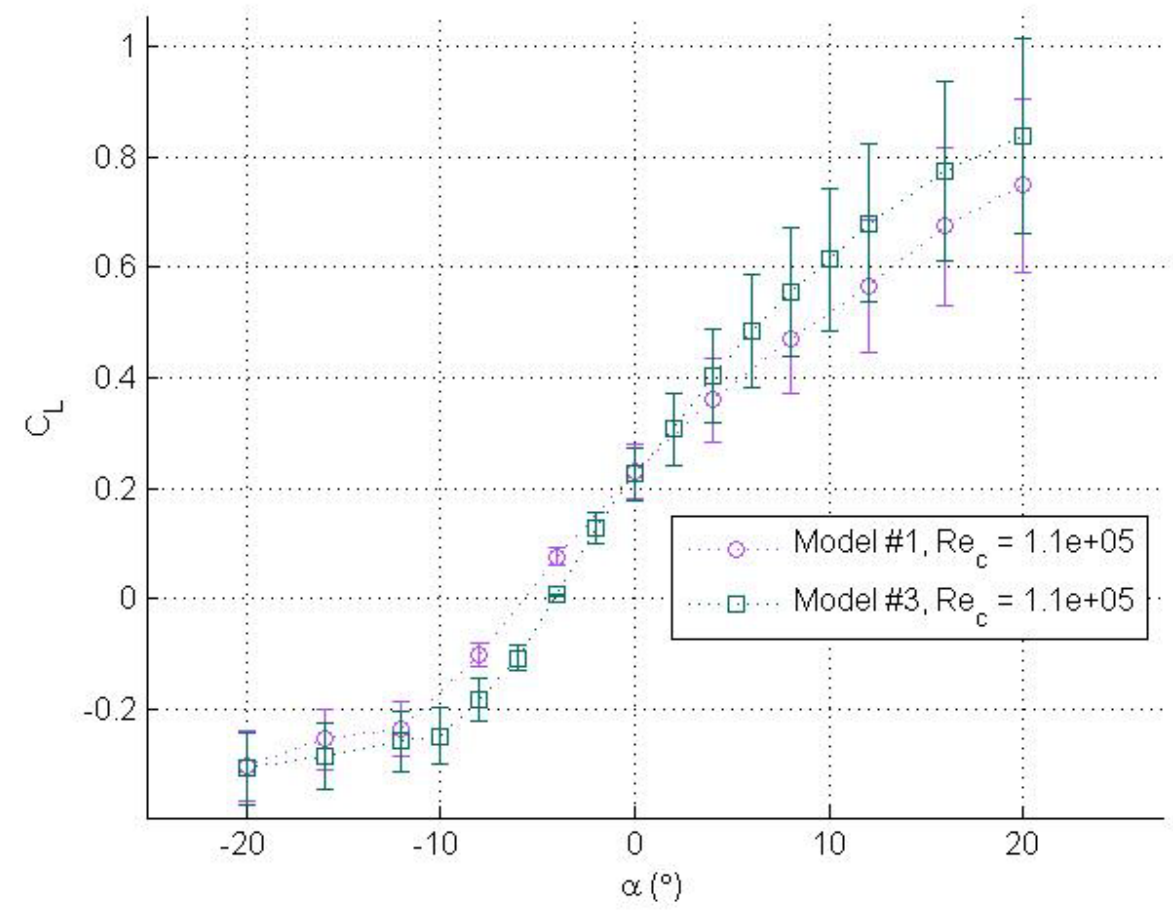

Figure 102: Comparison of the Differences in $C_{L}$ between the Preserved and Polyurethane Coated Models at 110,000 Re

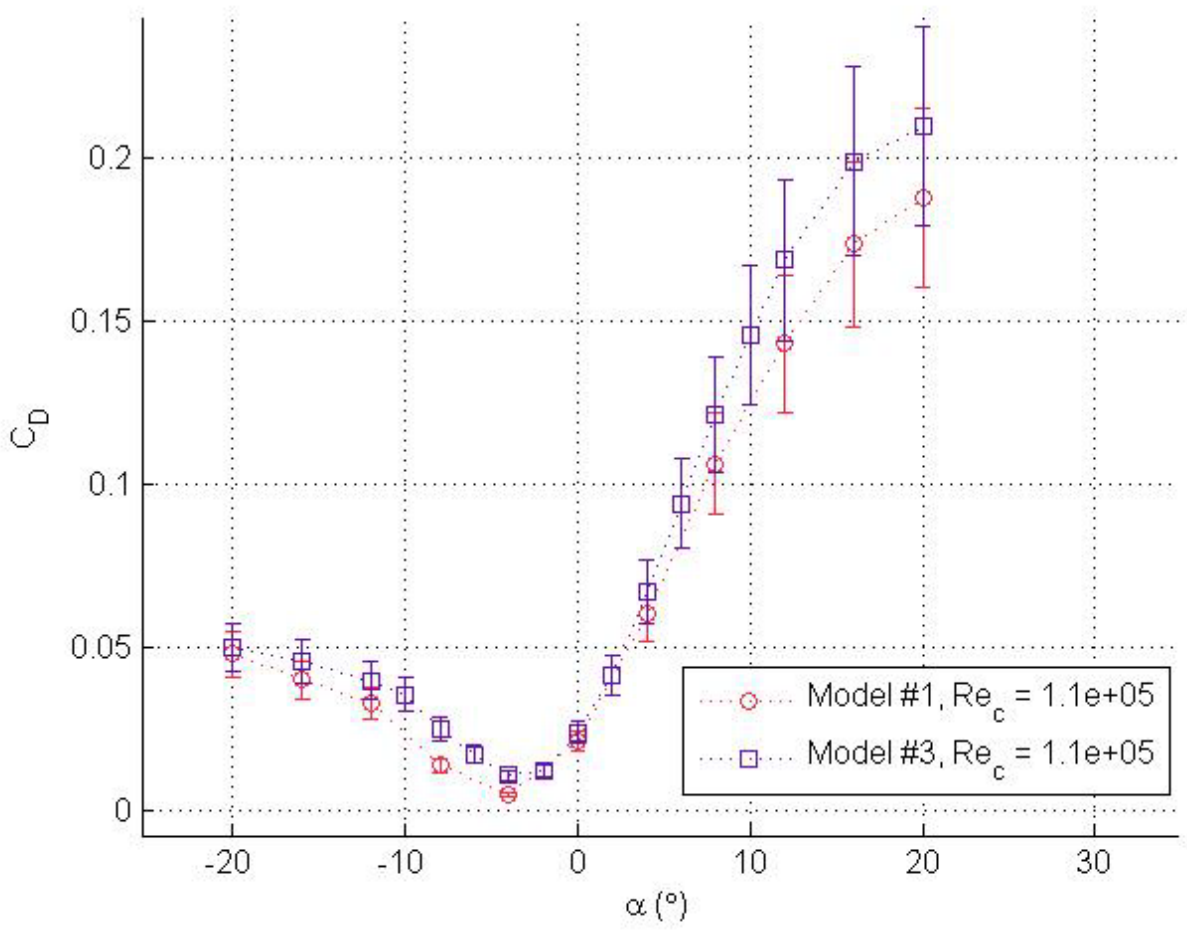

Figure 103: Comparison of the Differences in $C_{D}$ between the Preserved and Polyurethane Coated Models at 110,000 Re 
At $R e$ above 80,000, W3 continued to show a decrease in $C_{L}$ when compared to W1. However, these decreases occurred over a larger angle of attack range, from $-8^{\circ}$ to $-4^{\circ}$. $C_{D}$ values shown in Figure 96 for W3 were higher than W1 for the same angle of attack range.

The decreased performance of W3 corresponds with a decrease in secondary covert frequency and amplitude at $\alpha=0^{\circ}$ when compared to W1. At this angle of attack, both the coverts and secondary coverts were inhibited on W3. Primary and secondary feather motion was also drastically reduced at $\alpha=0^{\circ}$. The frequency of both covert feather groups was reduced by 91\% when compared to W1. The amplitude of the secondary feathers was also reduced, but the amplitude of the primary feathers was unaffected. 
Hair Sprayed Wing Model vs. Cast Wing Model

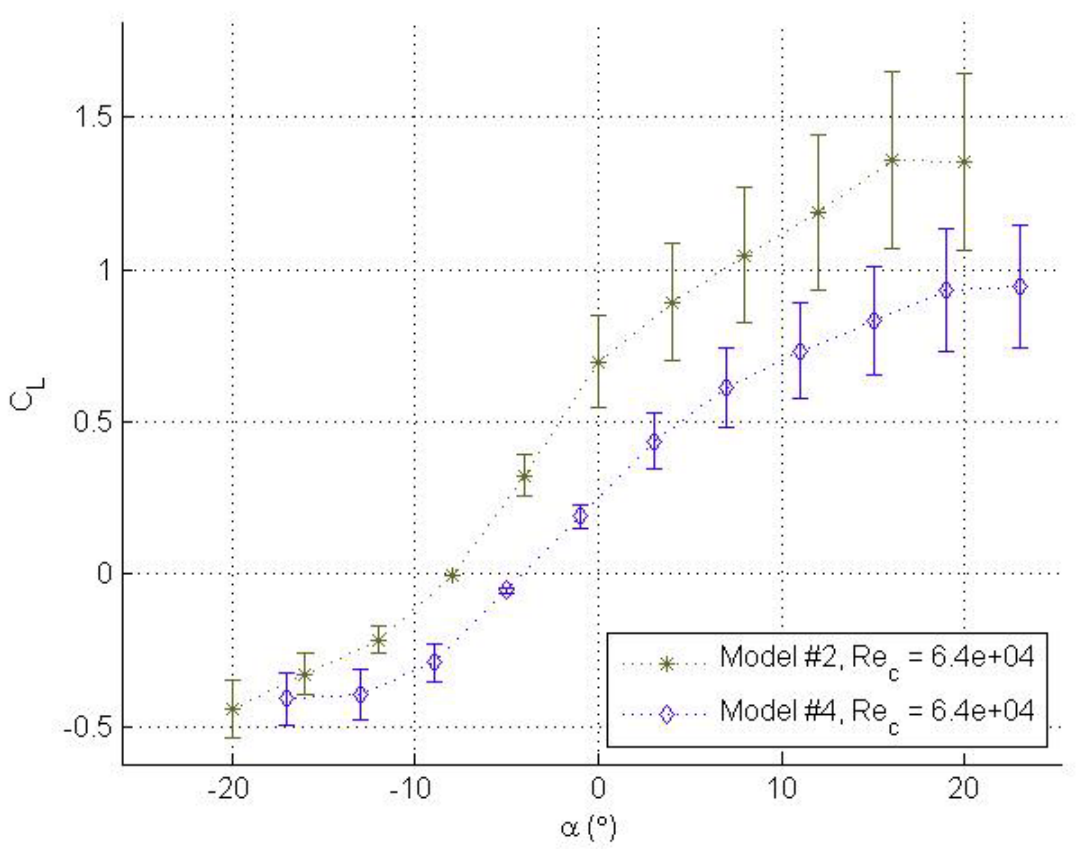

Figure 104: Comparison of the Differences in $C_{L}$ between the Hair Sprayed and Cast Wing Models at 64,000 Re

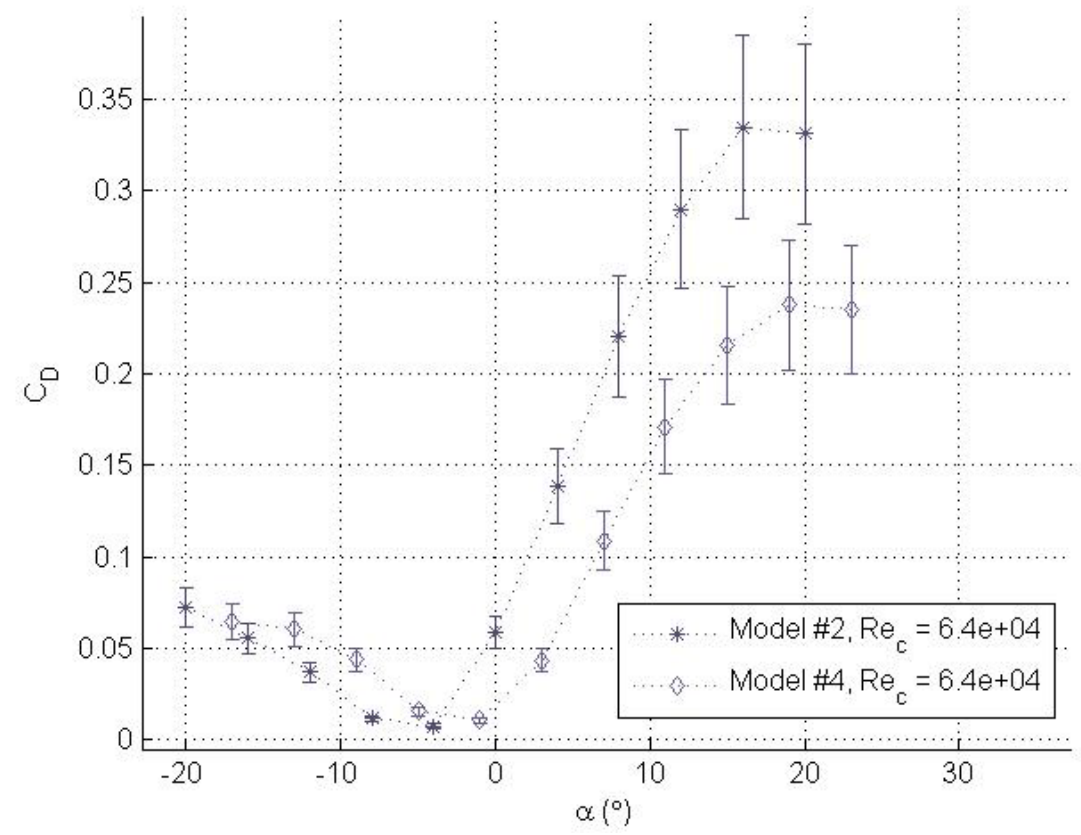

Figure 105: Comparison of the Differences in $C_{D}$ between the Hair Sprayed and Cast Wing Models at 64,000 Re 
When compared to W4, W2 showed higher $C_{L}$ values from $-12^{\circ}$ to $12^{\circ}$ angle of attack in Figure 106. W2 also exhibited higher $C_{D}$ values from $0^{\circ}$ to $24^{\circ}$ angle of attack. W2 did, however, have lower $C_{D}$ values between $-12^{\circ}$ and $-4^{\circ}$ angle of attack.

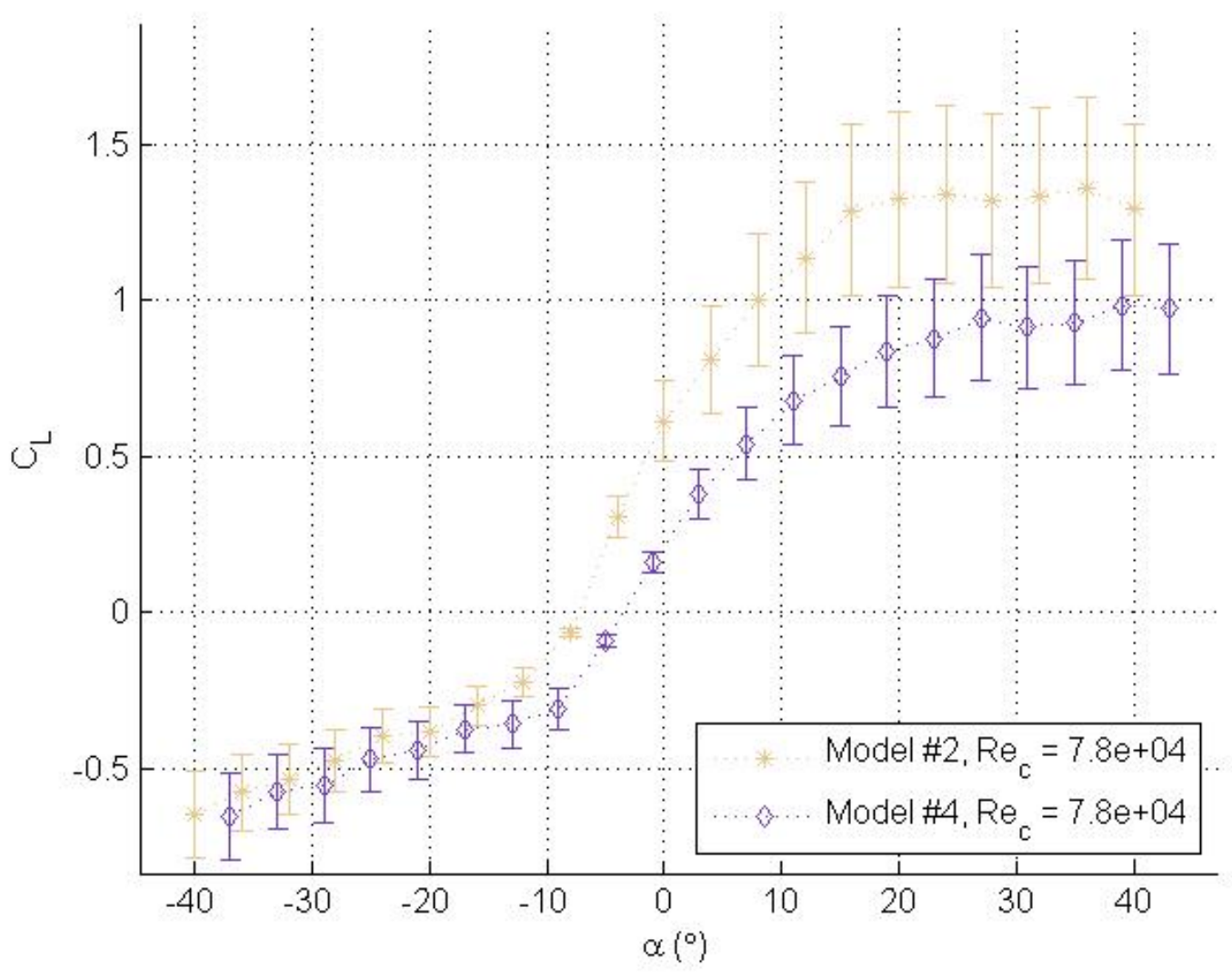

Figure 106: Comparison of the Differences in $C_{L}$ between the Hair Sprayed and Cast Wing Models at 78,000 Re 


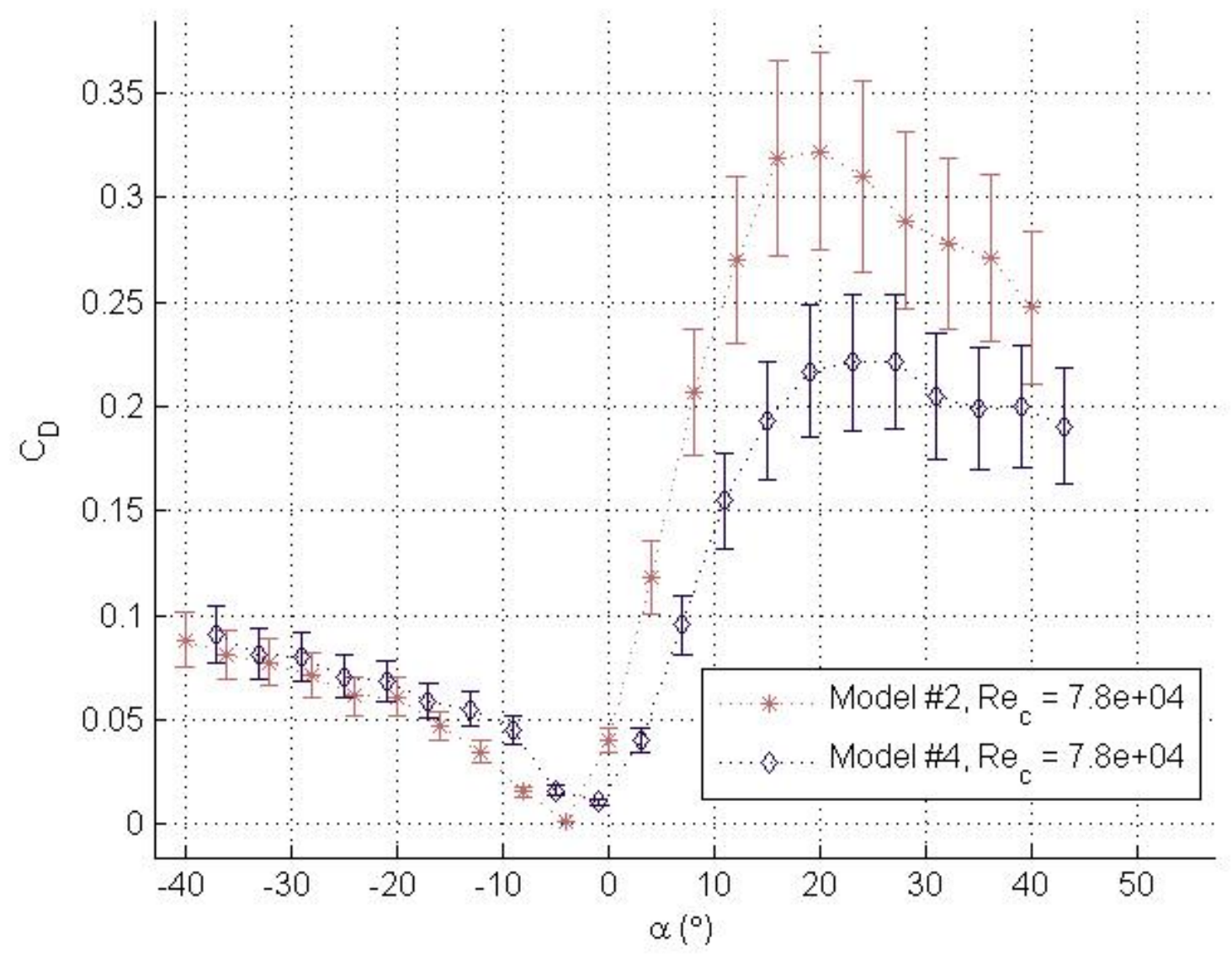

Figure 107: Comparison of the Differences in $C_{D}$ between the Hair Sprayed and Cast Wing Models at 78,000 Re

When the Re was increased from 63,000 to 78,000, the $C_{L}$ values for W3 decreased in the negative angle of attack range. As a result, the slope of the $C_{L}$ curve for W2 increased. The angle of zero lift shifted approximately $4^{\circ} \alpha$ between W2 and W4. However, this did not occur when W3 and W4 were compared. Therefore, this was not attributed to the experimental setup. 


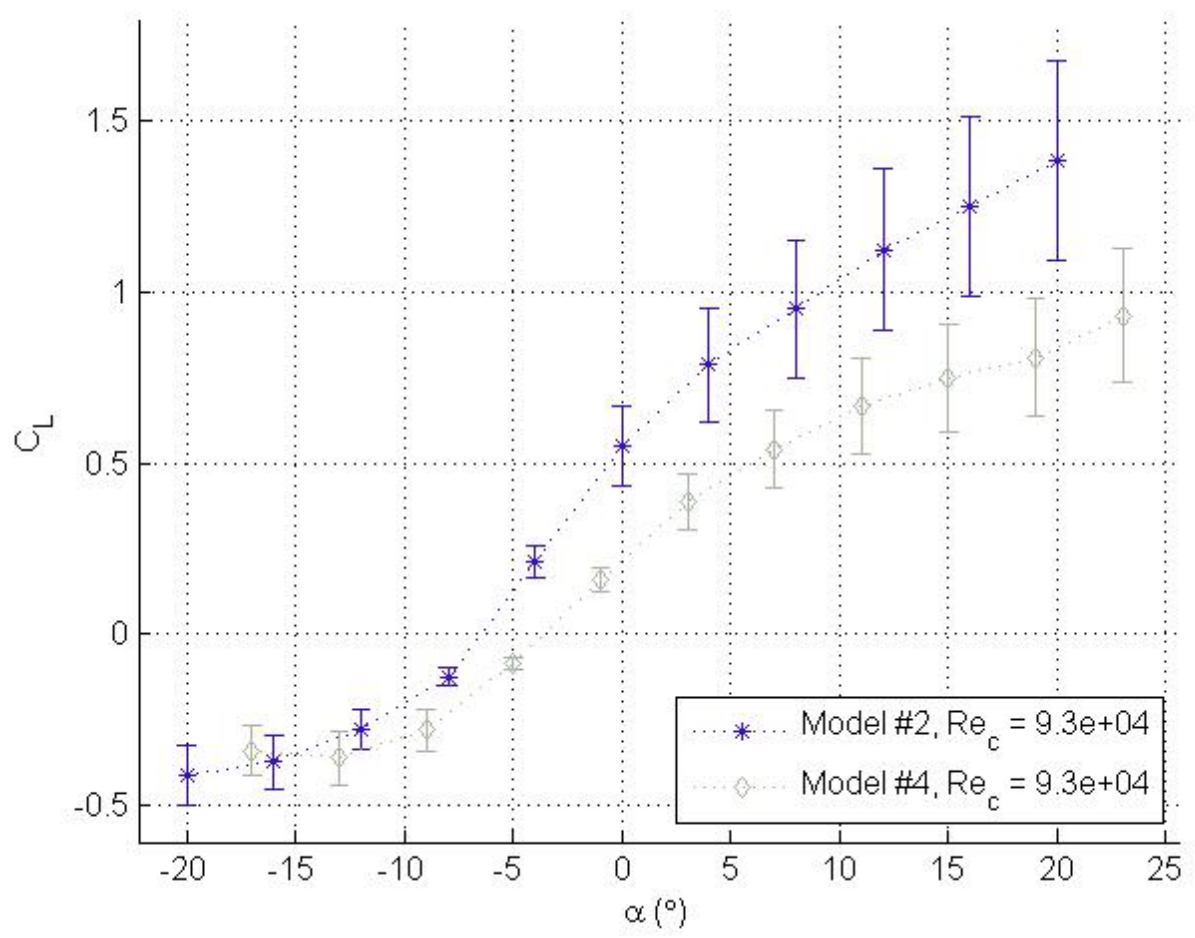

Figure 108: Comparison of the Differences in $C_{L}$ between the Hair Sprayed and Cast Wing Models at 93,000 Re

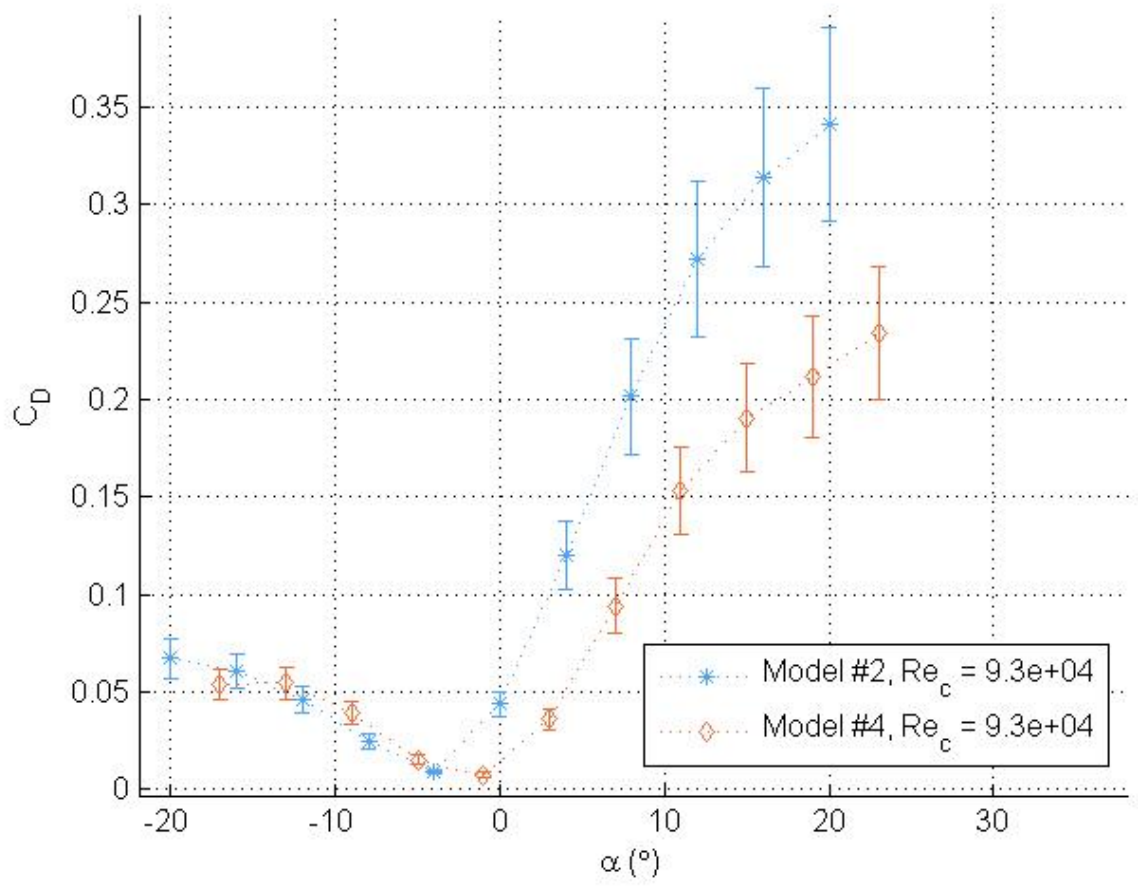

Figure 109: Comparison of the Differences in $C_{D}$ between the Hair Sprayed and Cast Wing Models at 93,000 Re 


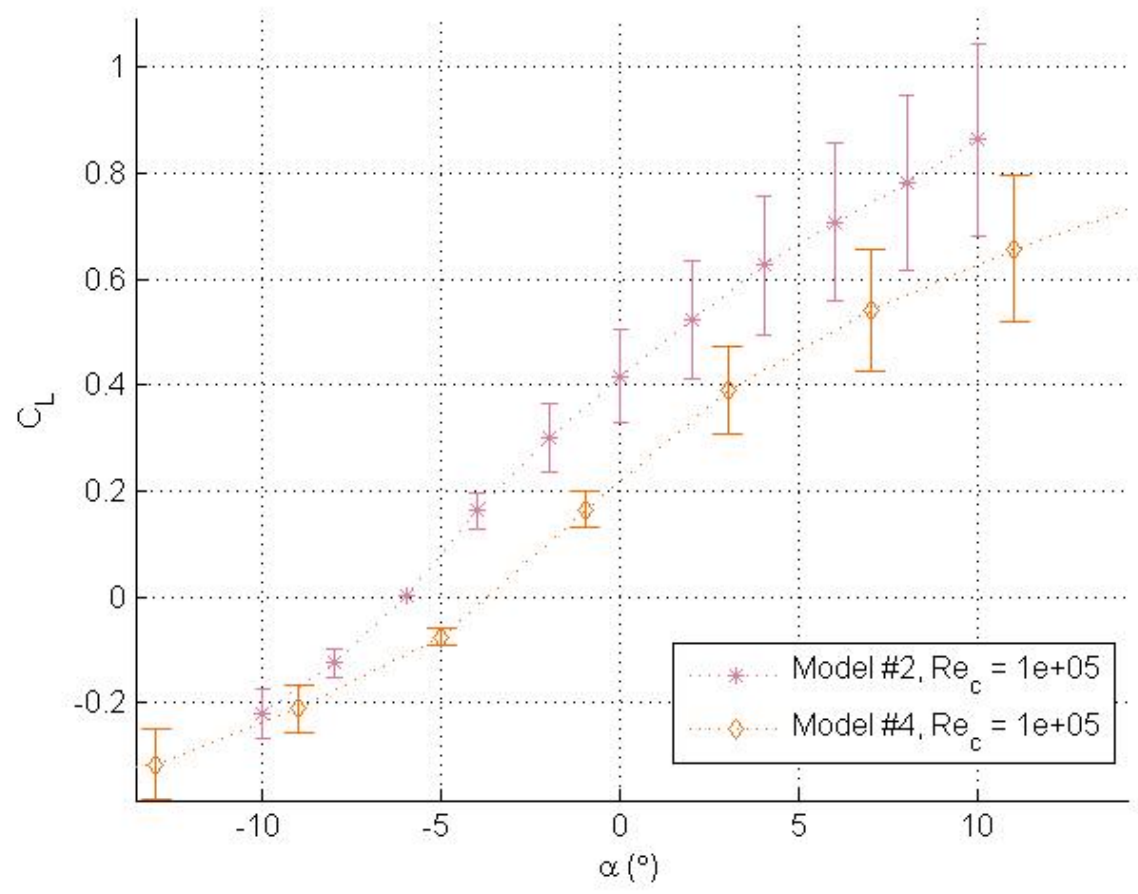

Figure 110: Comparison of the Differences in $C_{L}$ between the Hair Sprayed and Cast Wing Models at 100,000 Re

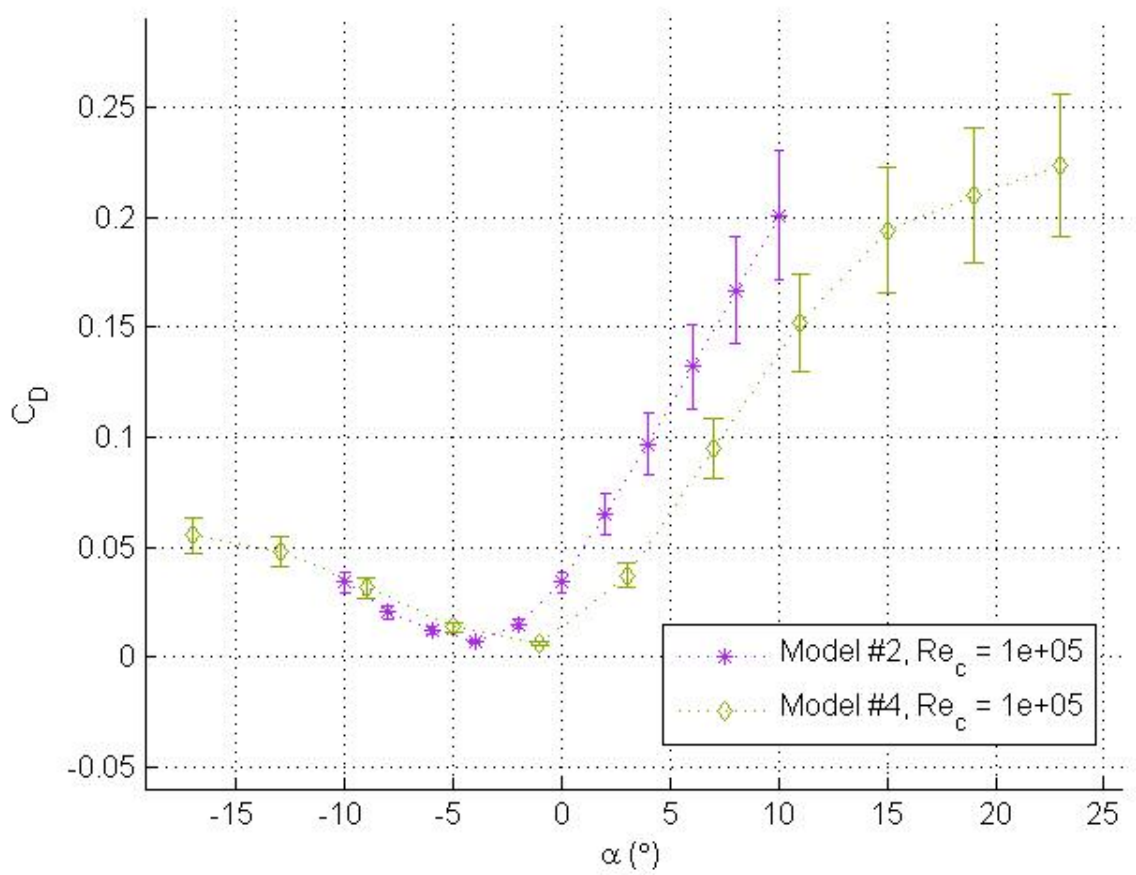

Figure 111: Comparison of the Differences in $C_{D}$ between the Hair Sprayed and Cast Wing Models at 100,000 Re 
The $C_{L}$ curve of W2 began to change as the $R e$ was increase beyond 80,000 to 93,000.

$C_{L}$ values begin to decrease and move the curve closer to the $C_{L}$ curve of W4 below $-8^{\circ}$ angle of attack. However, the $C_{L}$ values of W2 remain greater than those of W4 above $-8^{\circ}$ angle of attack. When the $R e$ was increased to 104,000 , the $C_{L}$ values of W2 increase and moved away from the $C_{L}$ curve of W4. A similar trend occurred for the $C_{D}$ curve of W2.

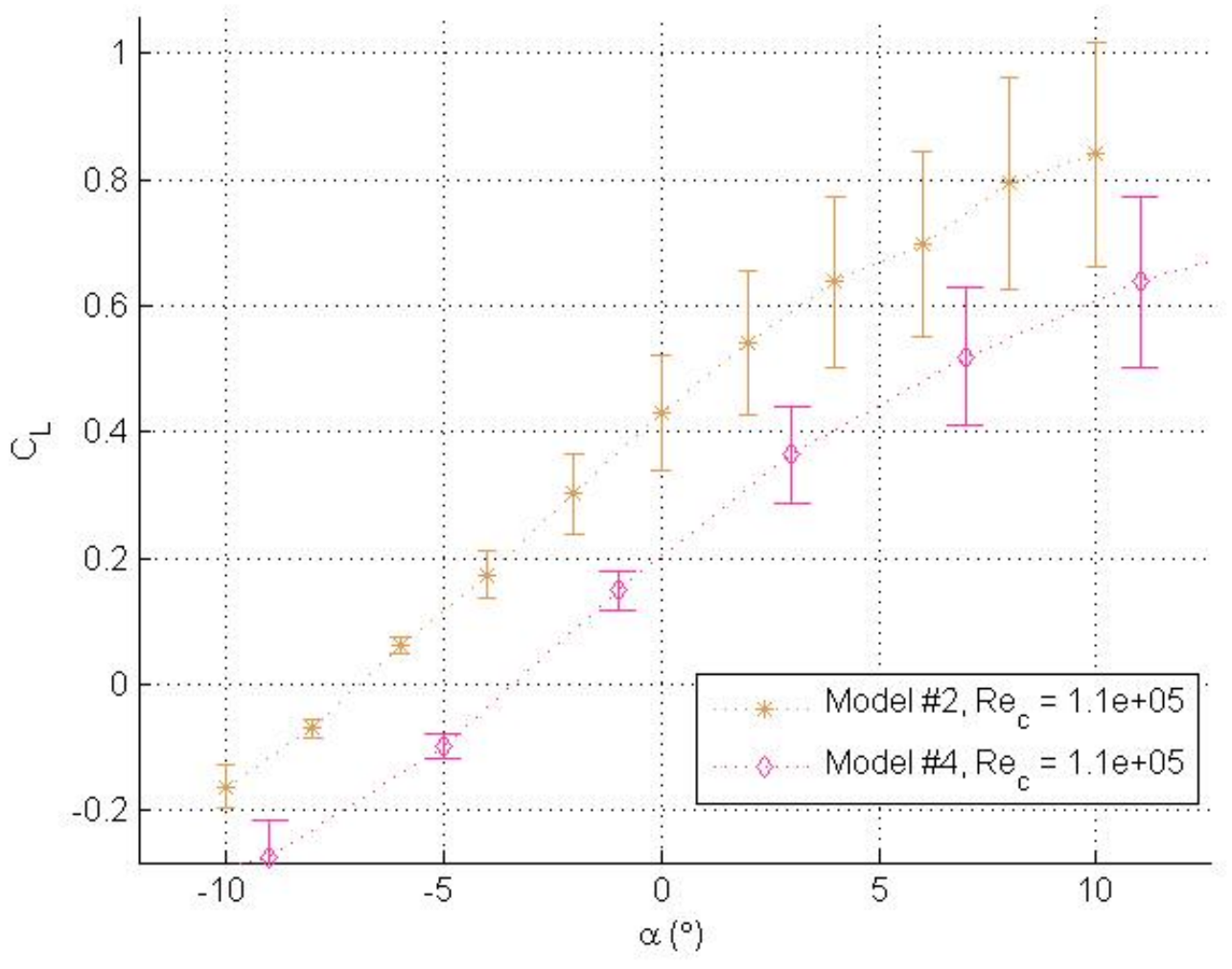

Figure 112: Comparison of the Differences in $C_{L}$ between the Hair Sprayed and Cast Wing Models at 110,000 Re 


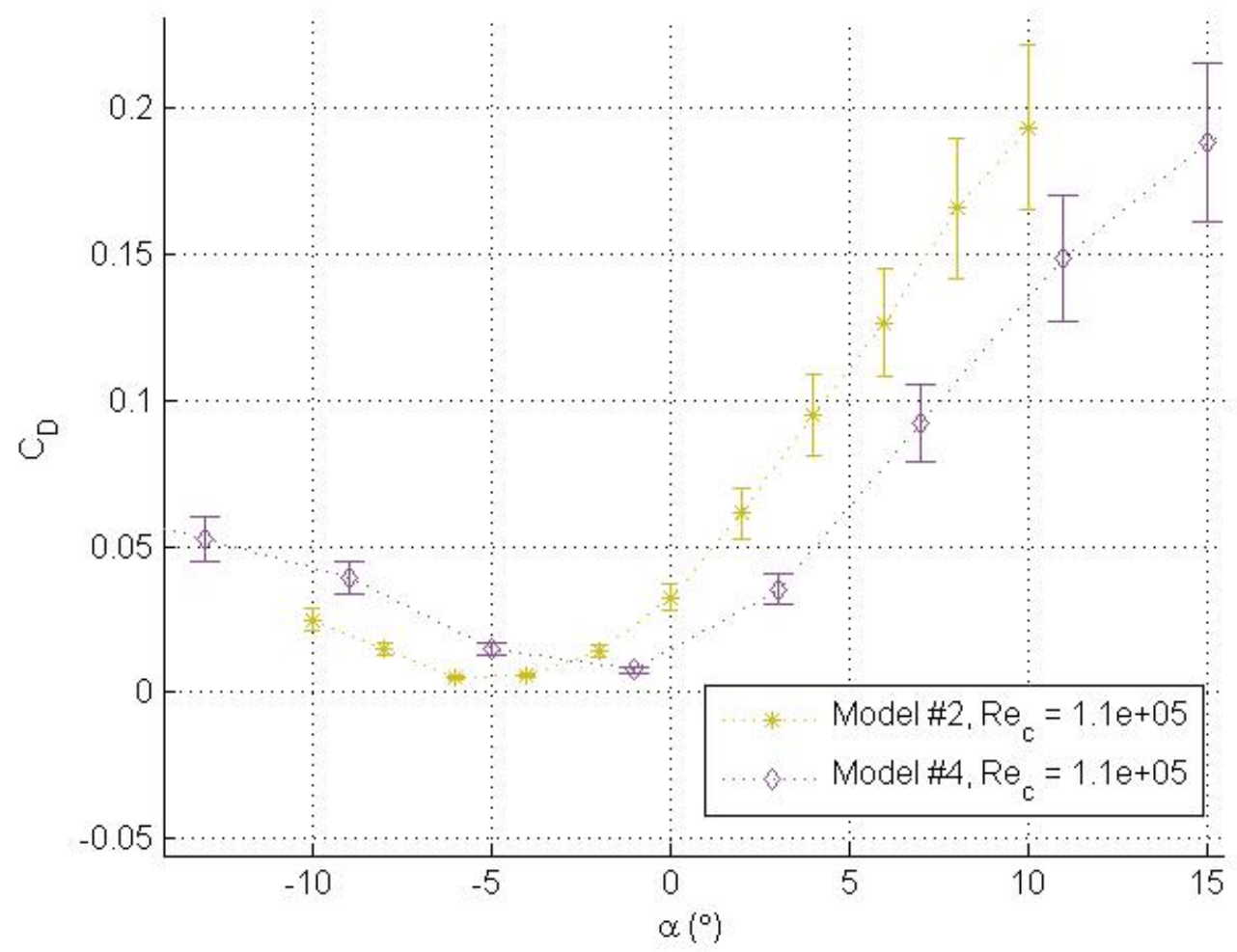

Figure 113: Comparison of the Differences in $C_{D}$ between the Hair Sprayed and Cast Wing Models at 110,000 Re

The increased performance of W2 begins at the same angles of attack that secondary covert feather frequency increases on W2 as seen in Figure 47. Increased performance for W2 continues as the angle of attack increases beyond $0^{\circ}$. At this point, secondary covert feather motion on $\mathrm{W} 2$ reduces and the covert feathers become active. Between $-12^{\circ}$ and $-4^{\circ}$, W2 shows lower $C_{D}$ values and higher $C_{L}$ values than $\mathrm{W} 4$ with no feather motion. 
W2 and W3 were compared to provide more information on the effects of feather motion. At $R e$ below 80,000, W2 showed higher $C_{L}$ values than W3 when the angle of attack was between $-8^{\circ}$ and $4^{\circ}$. Higher $C_{D}$ values were also exhibited by W2. These occur between $0^{\circ}$ and $4^{\circ}$ angle of attack.

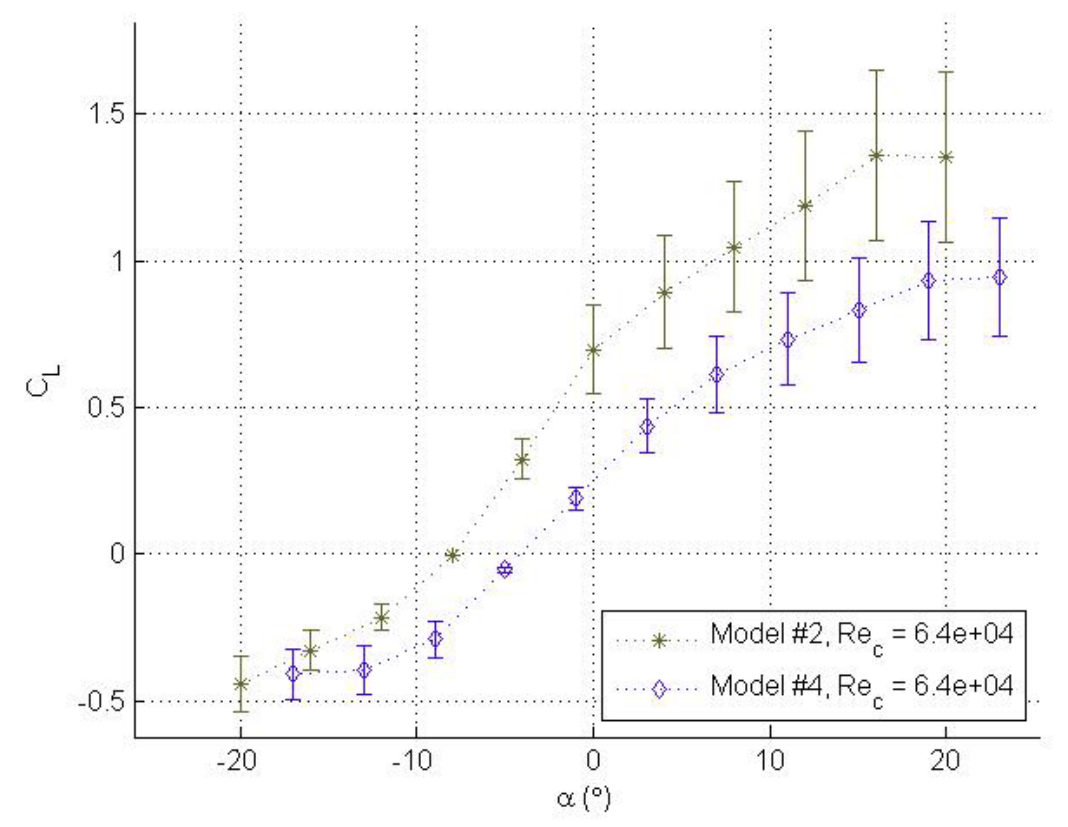

Figure 114: Comparison of the Differences in $C_{L}$ between the Hair Sprayed and Polyurethane Coated Wing Models at 64,000 Re 


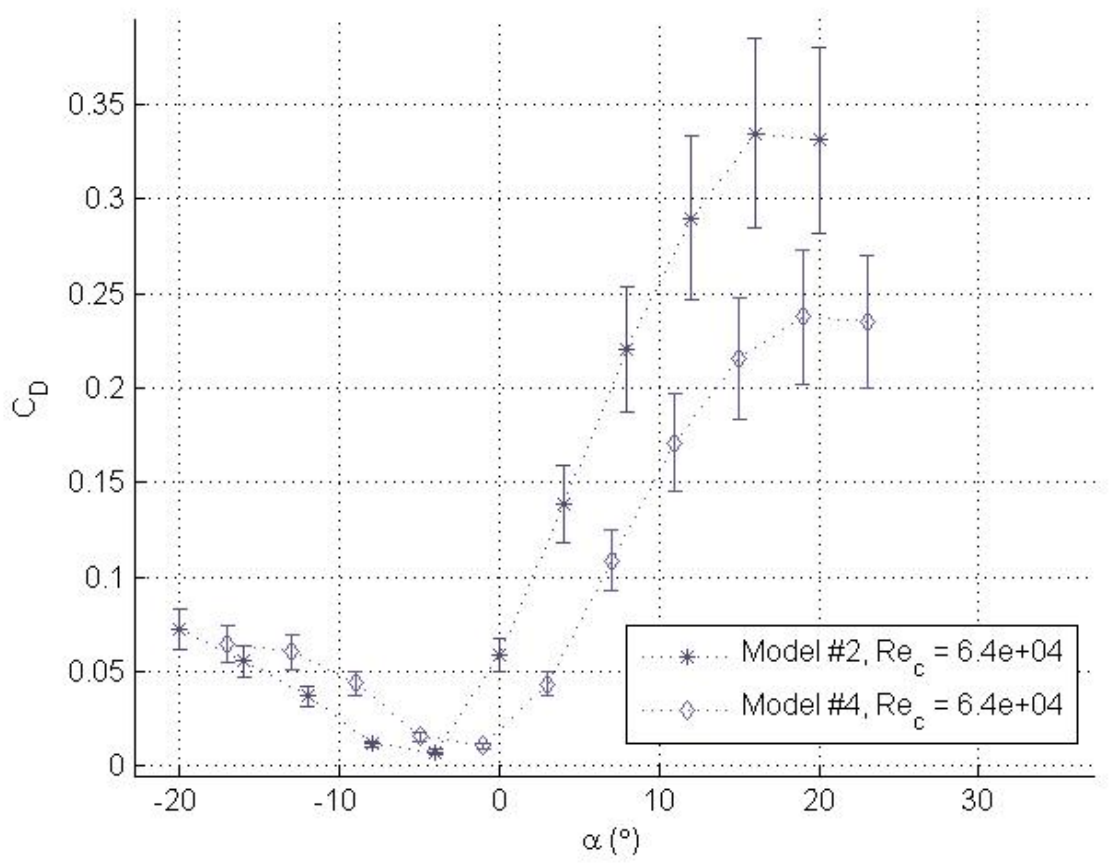

Figure 115: Comparison of the Differences in $C_{D}$ between the Hair Sprayed and Polyurethane Coated Wing Models at 64,000 Re

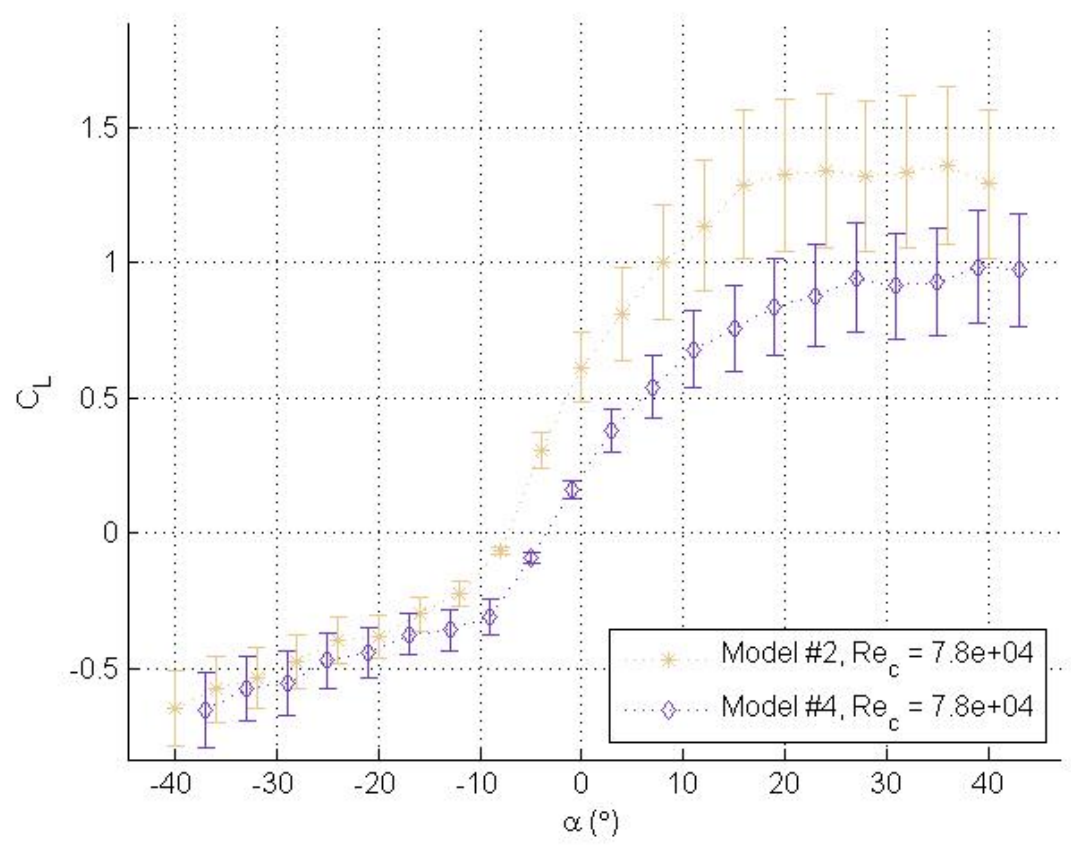

Figure 116: Comparison of the Differences in $C_{L}$ between the Hair Sprayed and Polyurethane Coated Wing Models at 78,000 Re 


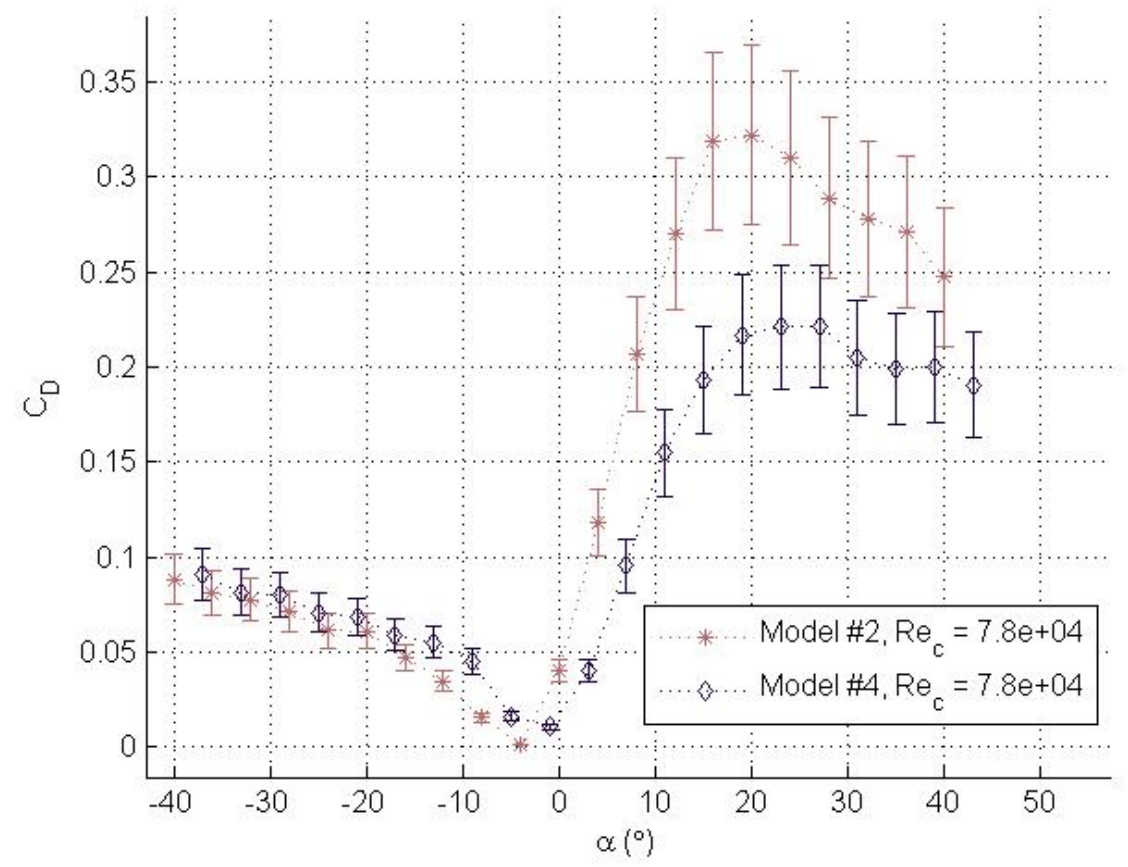

Figure 117: Comparison of the Differences in $C_{D}$ between the Hair Sprayed and Polyurethane Coated Wing Models at 78,000 Re

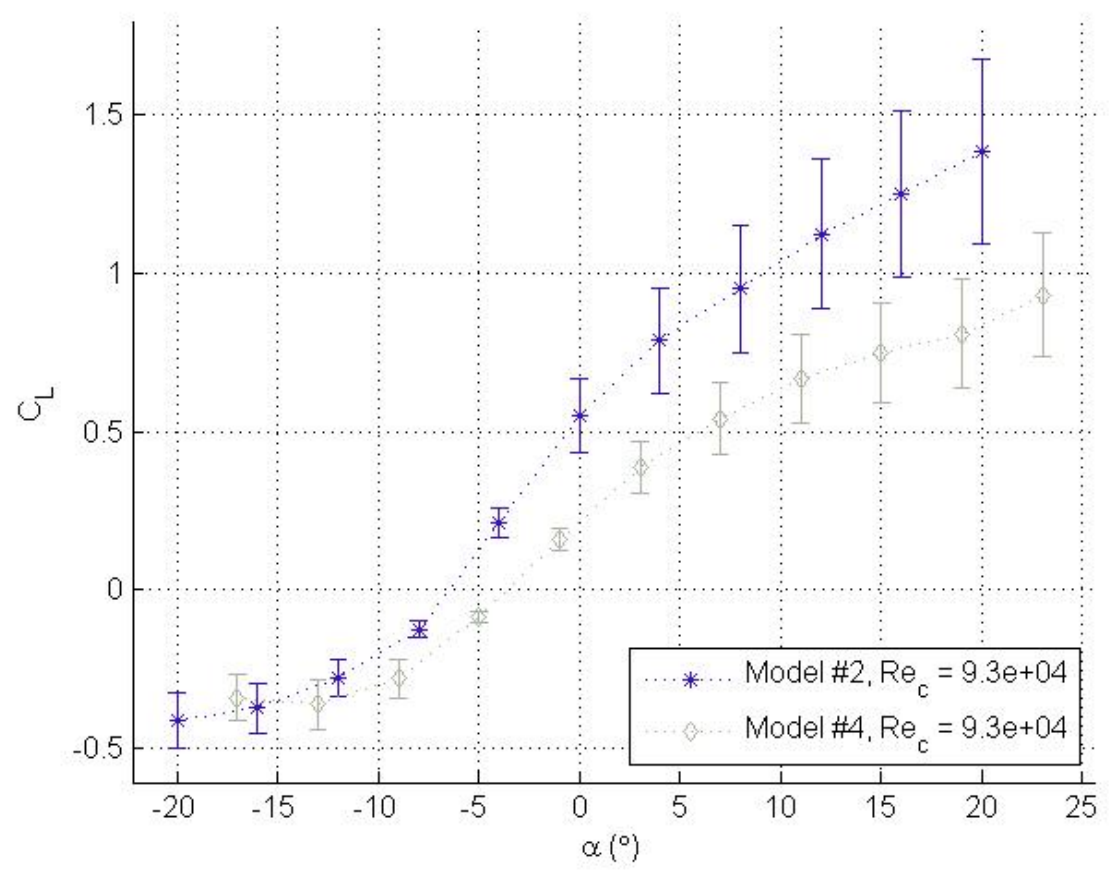

Figure 118: Comparison of the Differences in $C_{L}$ between the Hair Sprayed and Polyurethane Coated Wing Models at 93,000 Re 


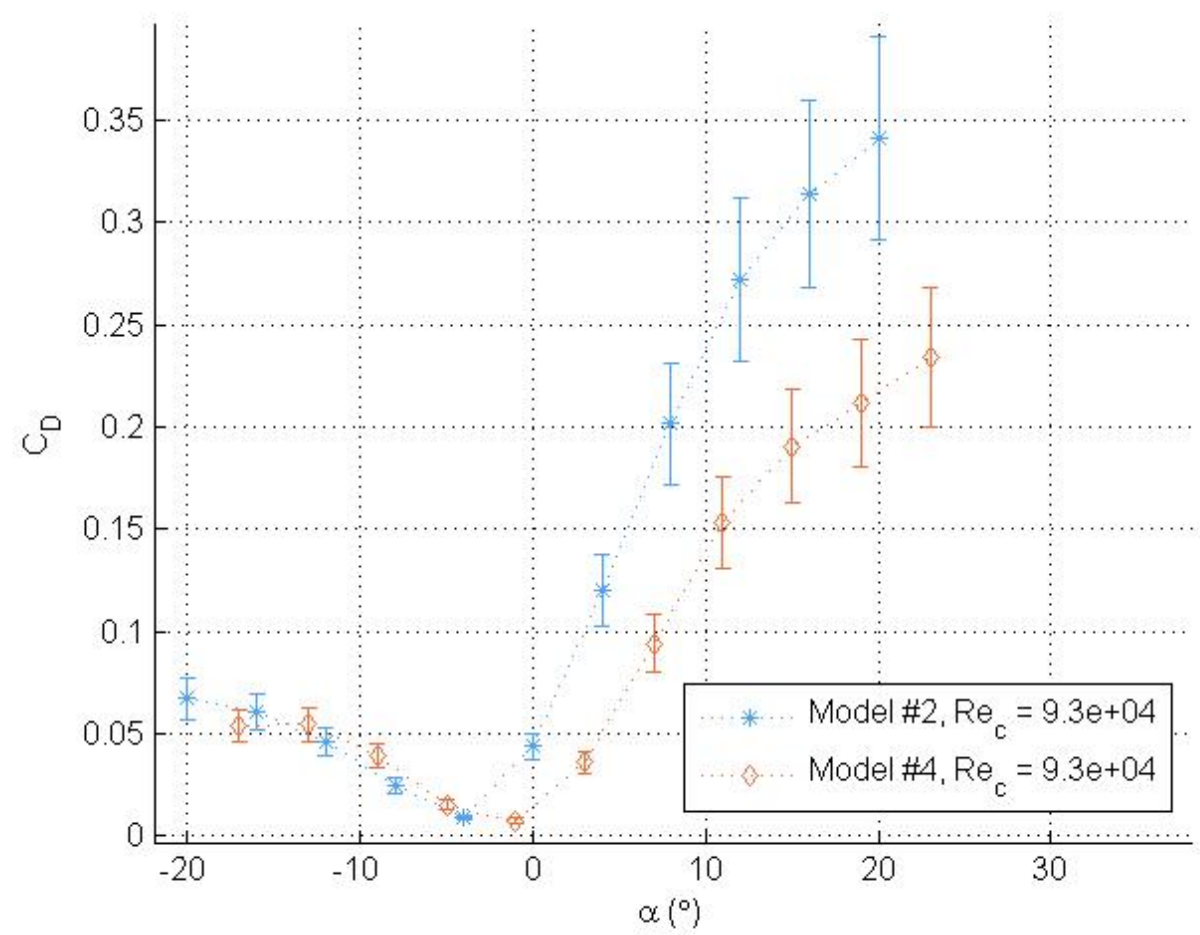

Figure 119: Comparison of the Differences in $C_{D}$ between the Hair Sprayed and Polyurethane Coated Wing Models at 93,000 Re

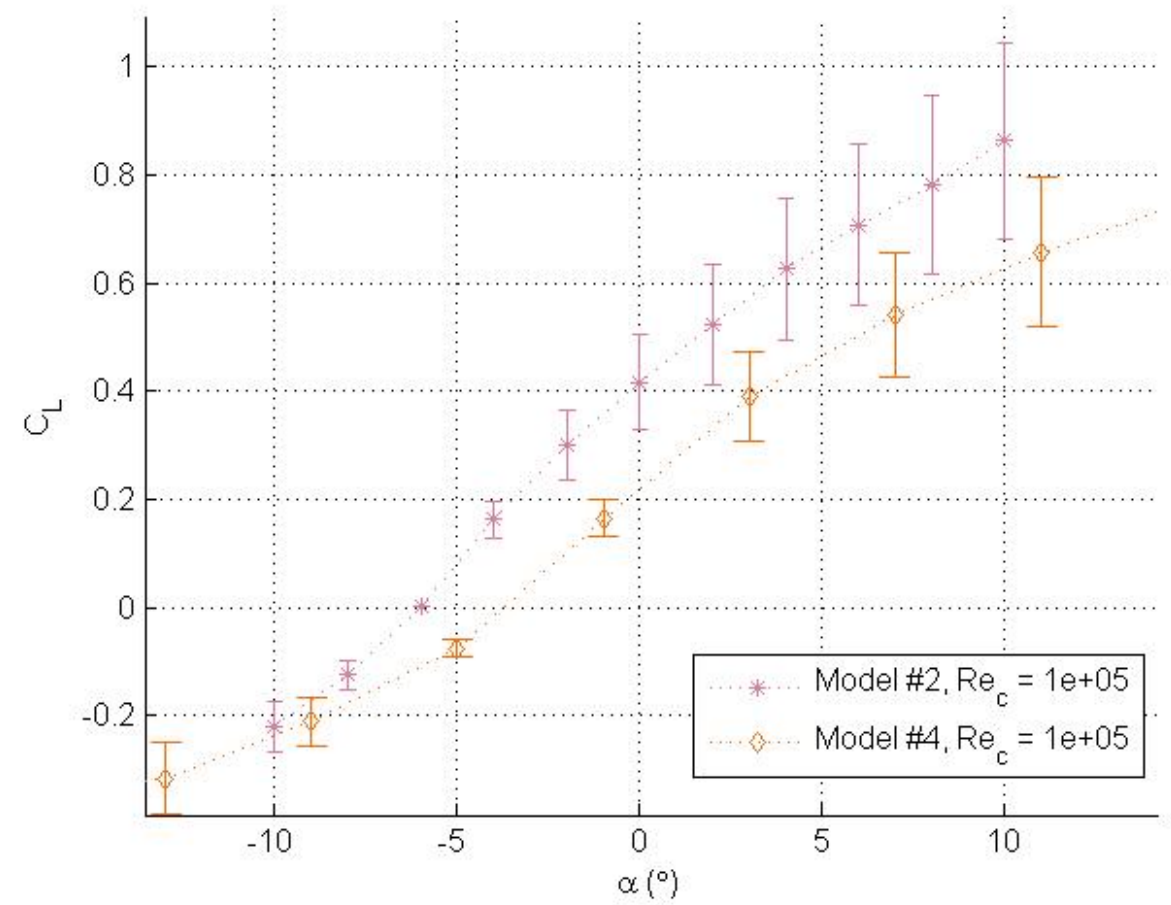

Figure 120: Comparison of the Differences in $C_{L}$ between the Hair Sprayed and Polyurethane Coated Wing Models at 100,000 Re 


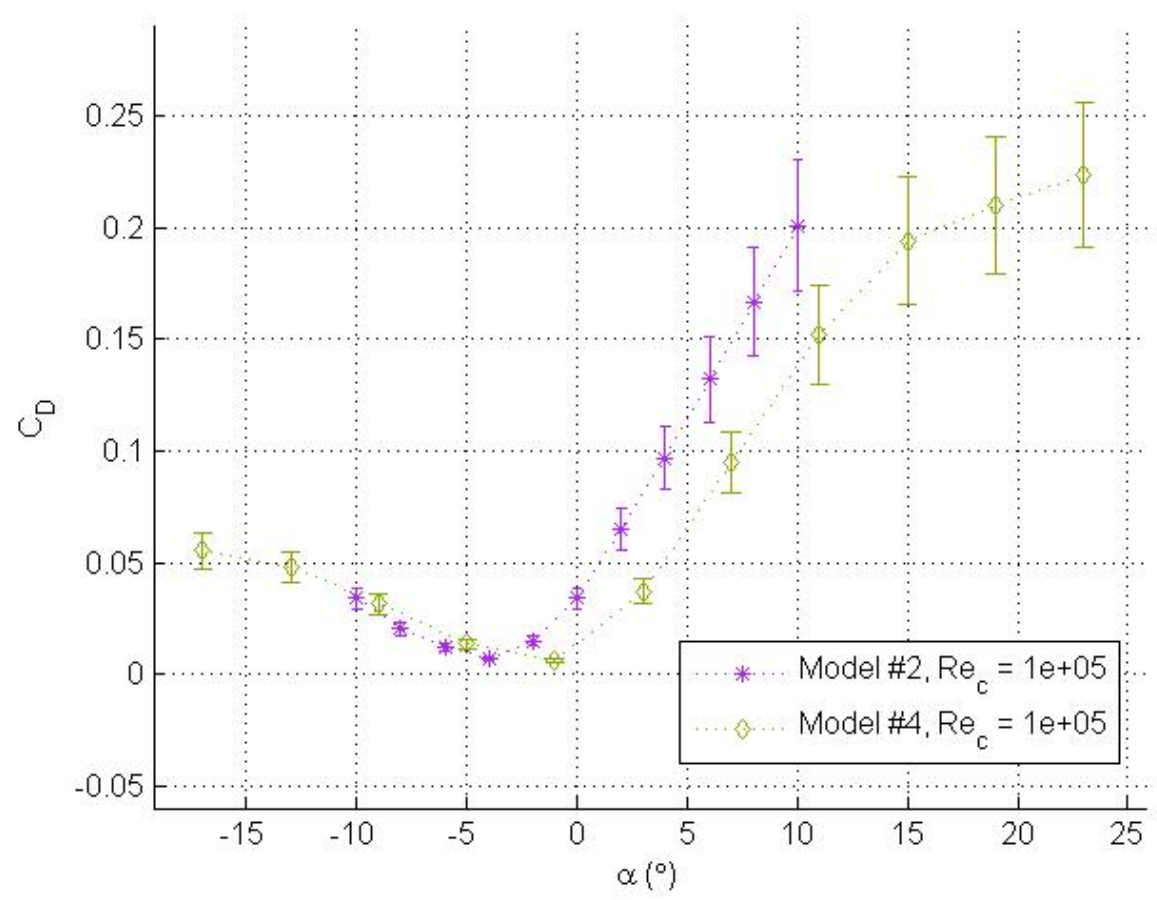

Figure 121: Comparison of the Differences in $C_{D}$ between the Hair Sprayed and Polyurethane Coated Wing Models at 100,000 Re

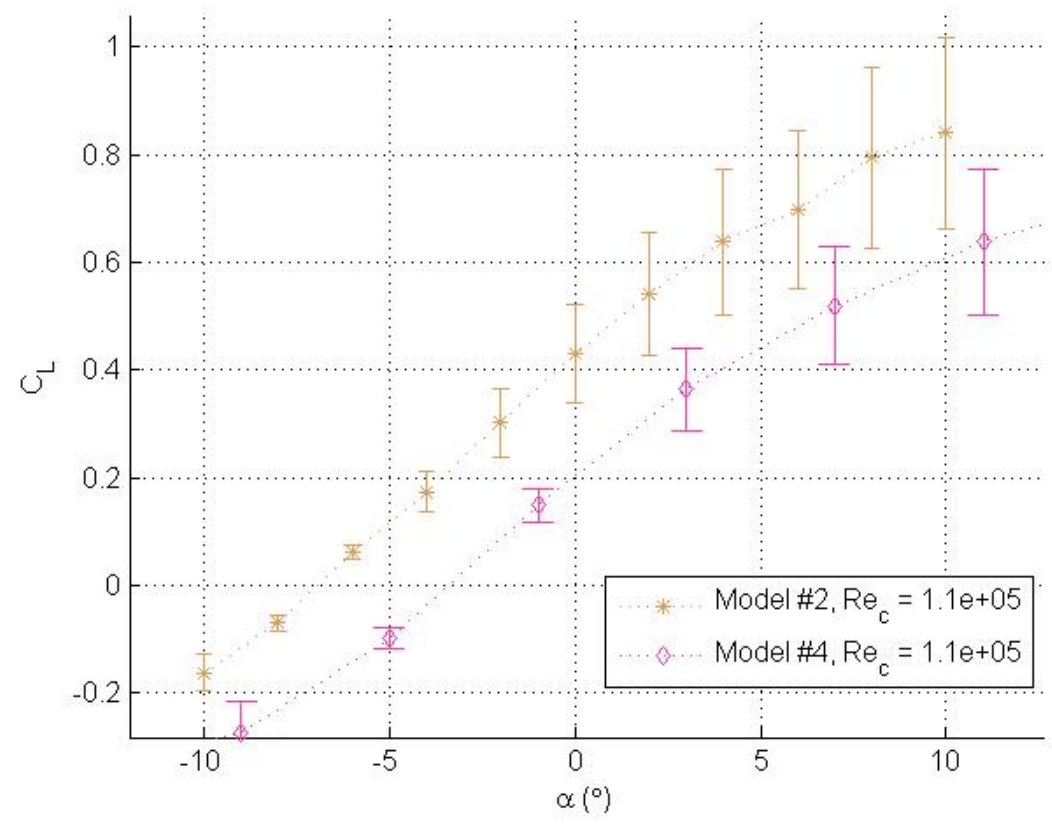

Figure 122: Comparison of the Differences in $C_{L}$ between the Hair Sprayed and Polyurethane Coated Wing Models at 110,000 Re 


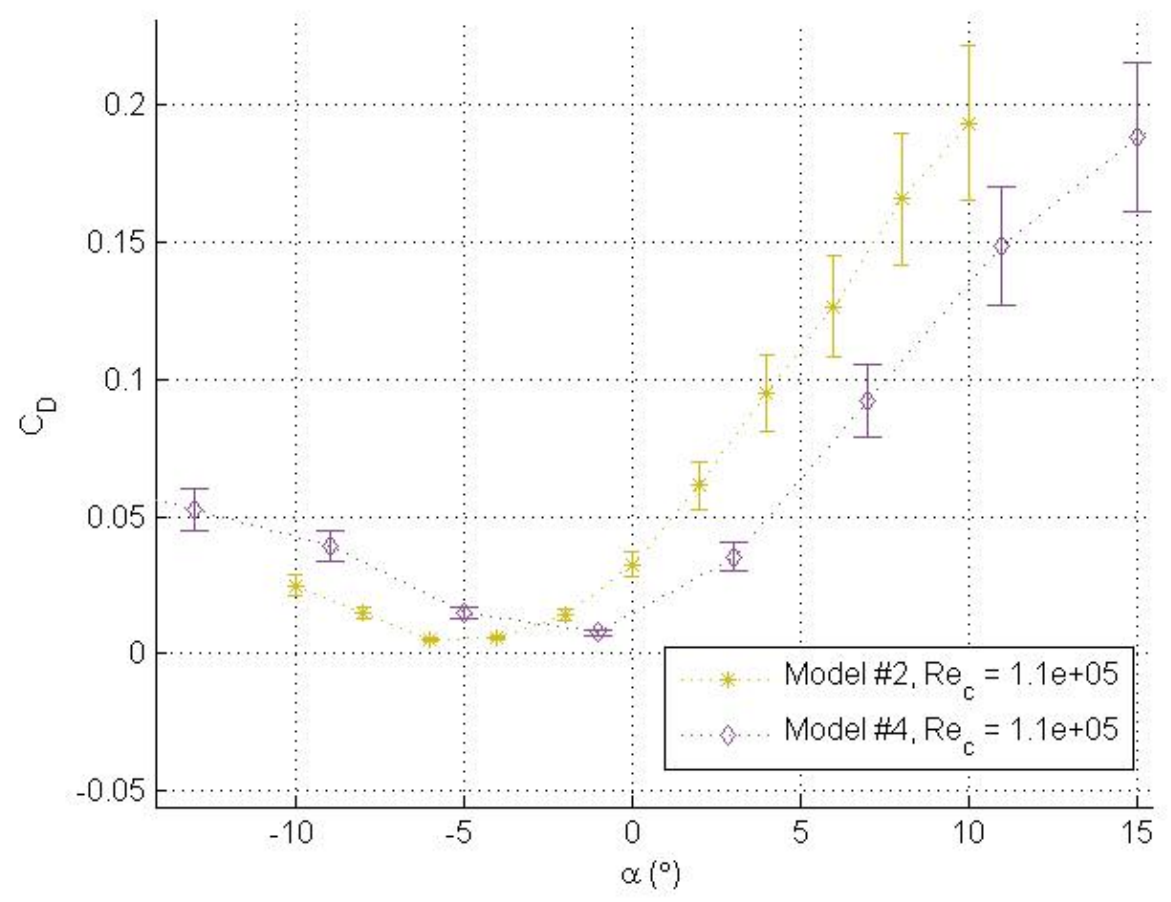

Figure 123: Comparison of the Differences in $C_{D}$ between the Hair Sprayed and Polyurethane Coated Wing Models at 110,000 Re

W2 continued to outperform W3 at $R e$ above 80,000. W2 showed increased $C_{L}$ values when compared to W3. However, the angle of attack range at which this became certain decreased from $-4^{\circ}$ to $-10^{\circ}$ as the $R e$ was increased to 109,000 . The increase in $C_{L}$ values ended at $6^{\circ}$ angle of attack for this $R e$ range.

Increases in $C_{D}$ were also noticed when the angle of attack was above $4^{\circ}$. The difference in the $C_{D}$ values of W2 and W3 decreased as the Re was increased. However, at 109,000 Re, the $C_{D}$ for W2 was lower than W3 for $-10^{\circ}$ to $-4^{\circ}$ angle of attack. W2 showed an increase in $C_{L}$ under the same conditions. 


\subsubsection{Effects of Surface Geometry}

Cast Wing Model vs. Plasti Dipped Wing Model

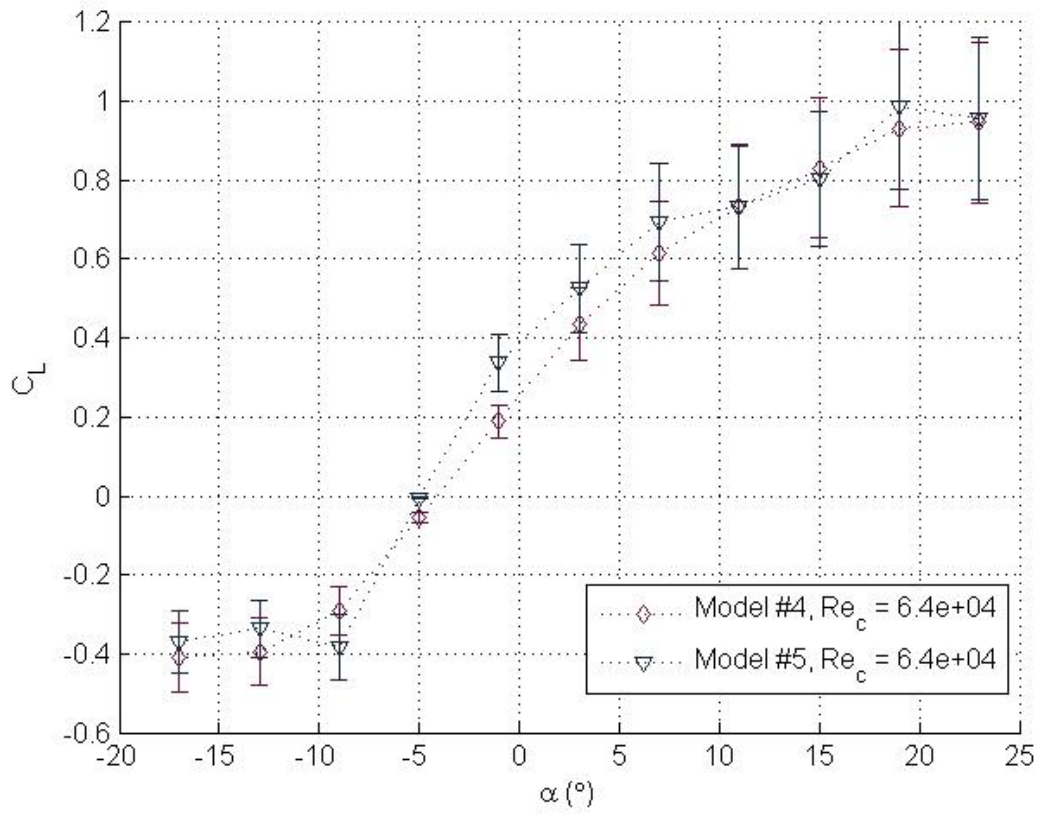

Figure 124: Comparison of the Differences in $C_{L}$ between the Cast and Plasti Dipped Wing Models at 64,000 Re

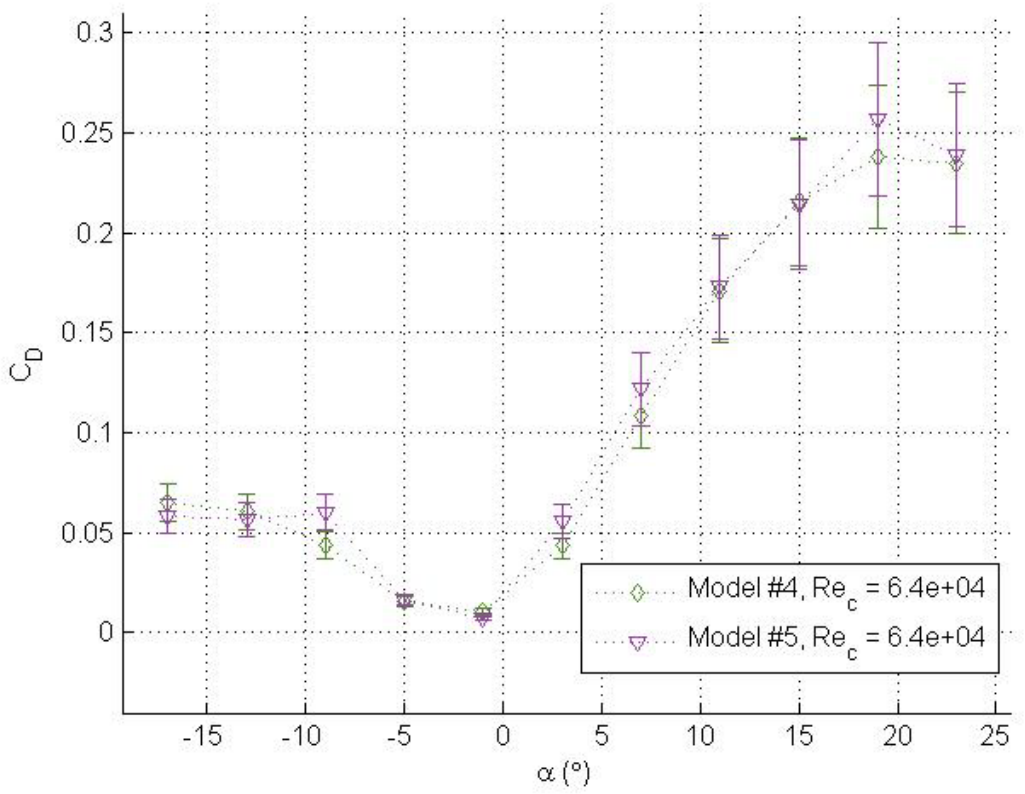

Figure 125: Comparison of the Differences in $C_{D}$ between the Cast and Plasti Dipped Wing Models at 64,000 Re 
The primary change to the surface geometry between W4 and W5 was the depth of the surface features. The application of Plasti Dip served to fill in the fine surface features of the wing. Figure 124 shows the $C_{L}$ and $C_{D}$ curves were only affected at $64,000 R e$. At this $R e$, W5 exhibited an increased $C_{L}$ value from $-5^{\circ}$ to $-1^{\circ} \alpha$. $C_{D}$ was also increased at $-8^{\circ}$ and $3^{\circ} \alpha$.

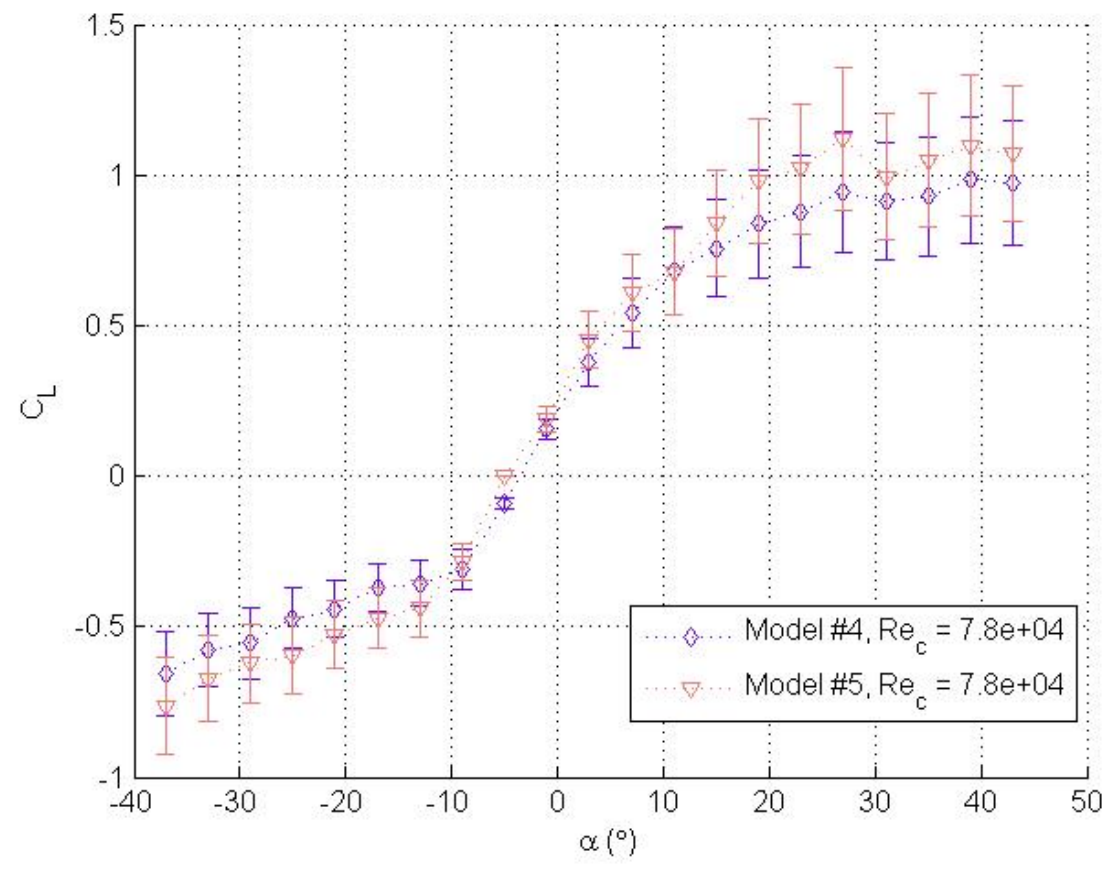

Figure 126: Comparison of the Differences in $C_{L}$ between the Cast and Plasti Dipped Wing Models at 78,000 Re 


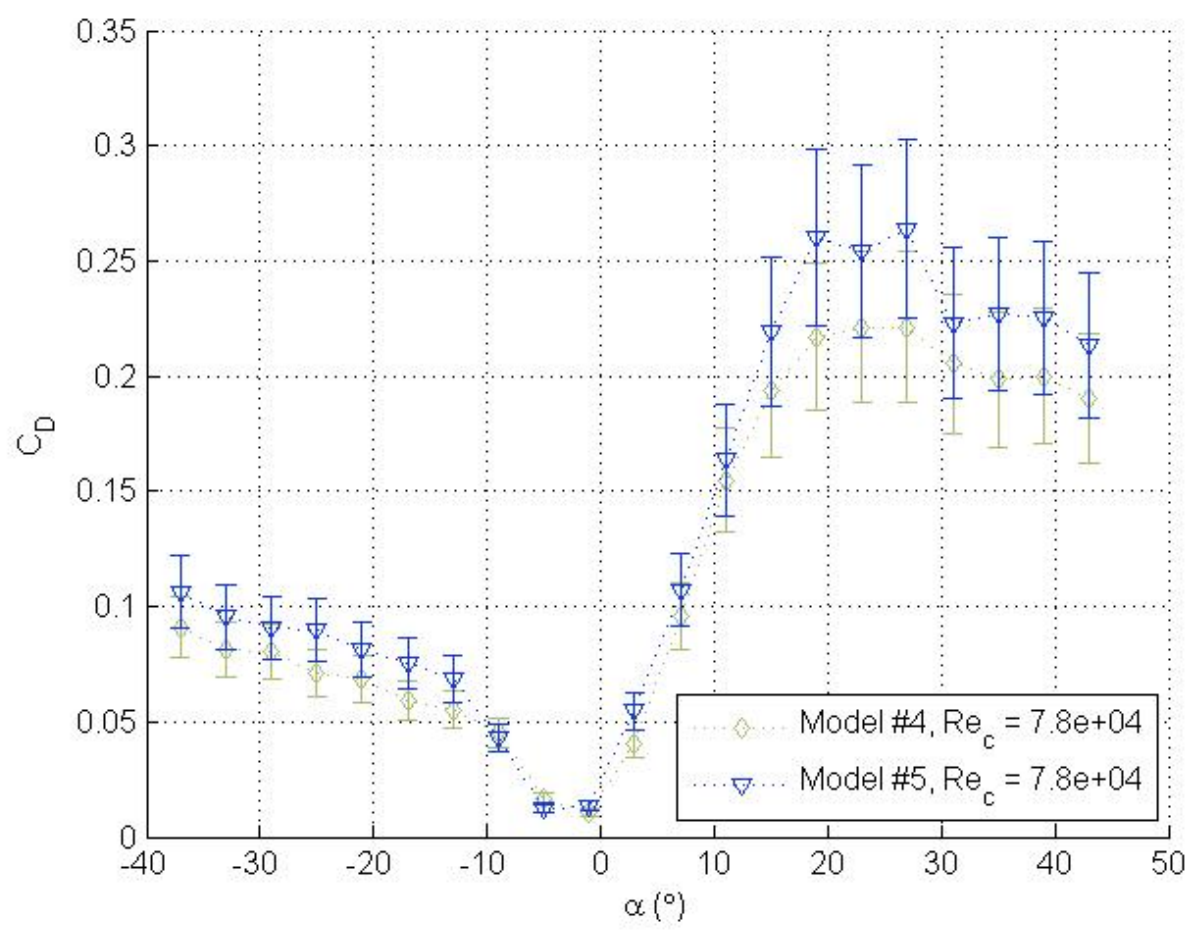

Figure 127: Comparison of the Differences in $C_{D}$ between the Cast and Plasti Dipped Wing Models at 78,000 Re

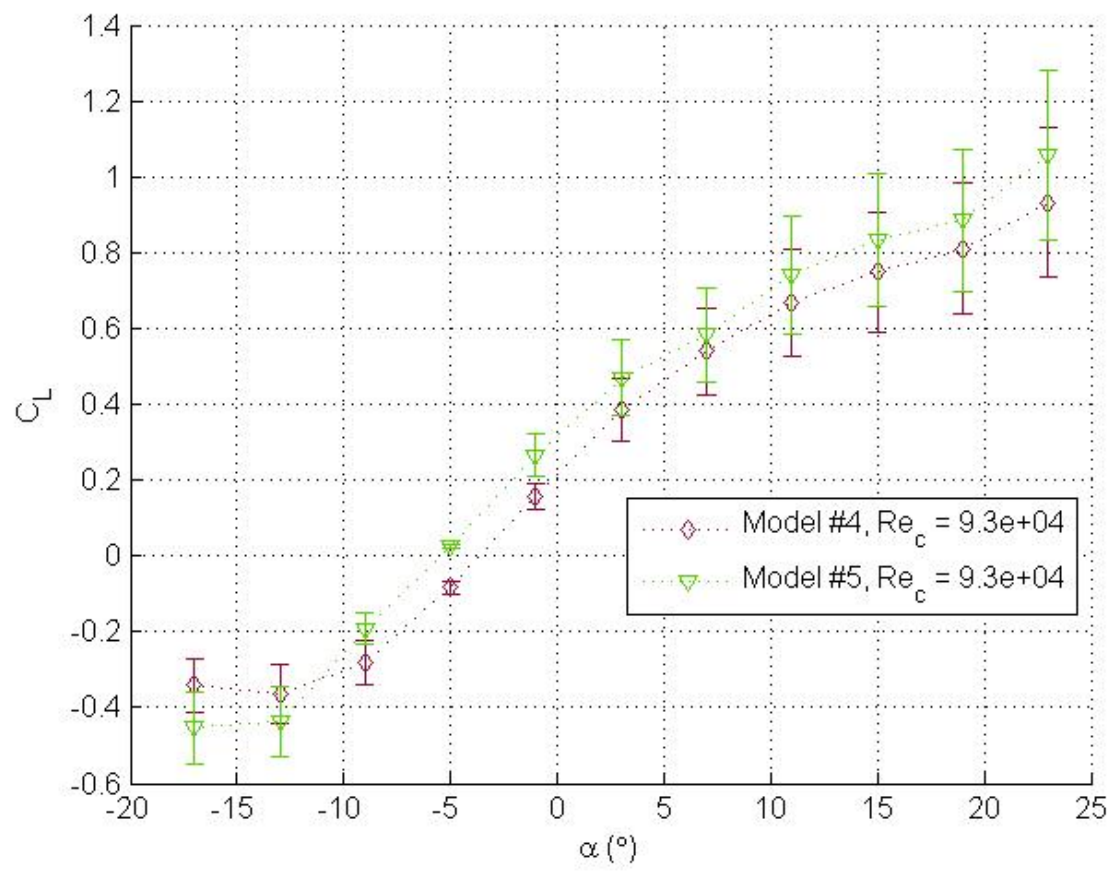

Figure 128: Comparison of the Differences in $C_{L}$ between the Cast and Plasti Dipped Wing Models at 93,000 Re 


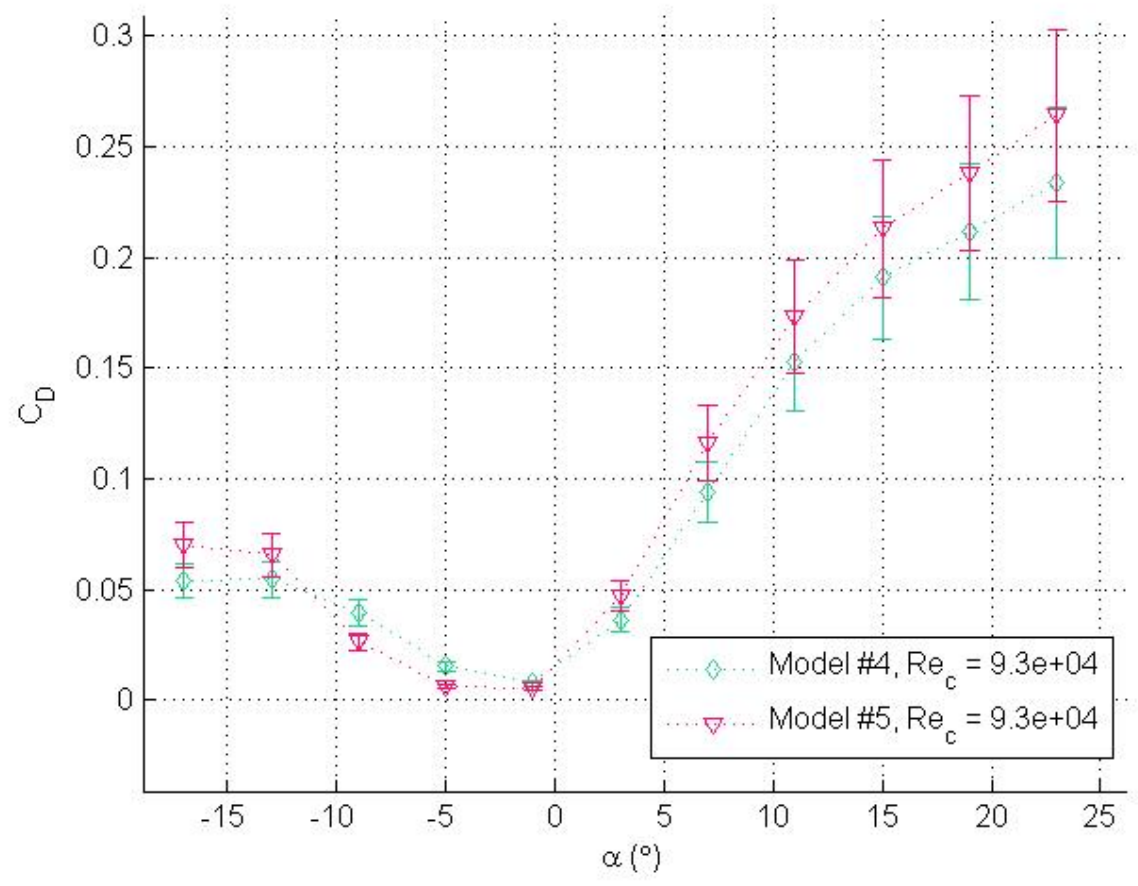

Figure 129: Comparison of the Differences in $C_{D}$ between the Cast and Plasti Dipped Wing Models at 93,000 Re

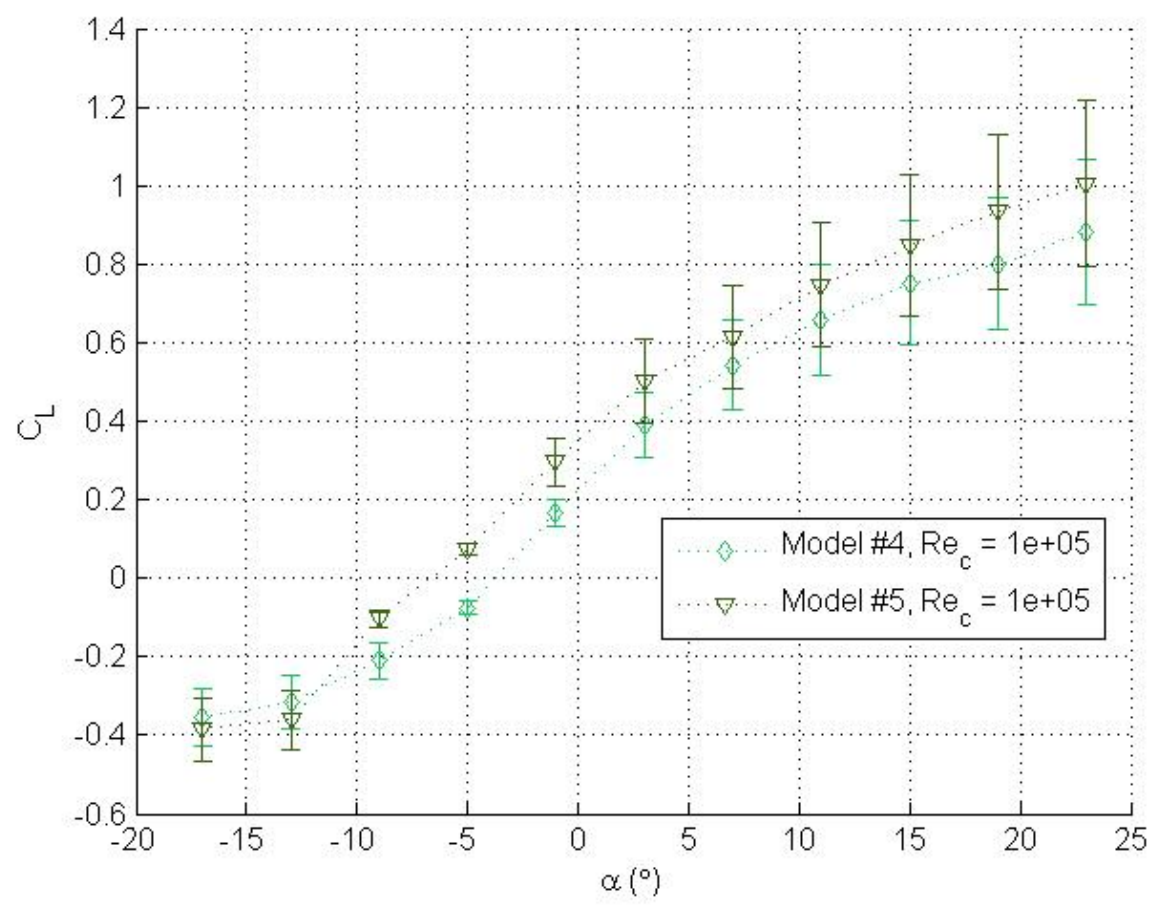

Figure 130: Comparison of the Differences in $C_{L}$ between the Cast and Plasti Dipped Wing Models at 100,000 Re 


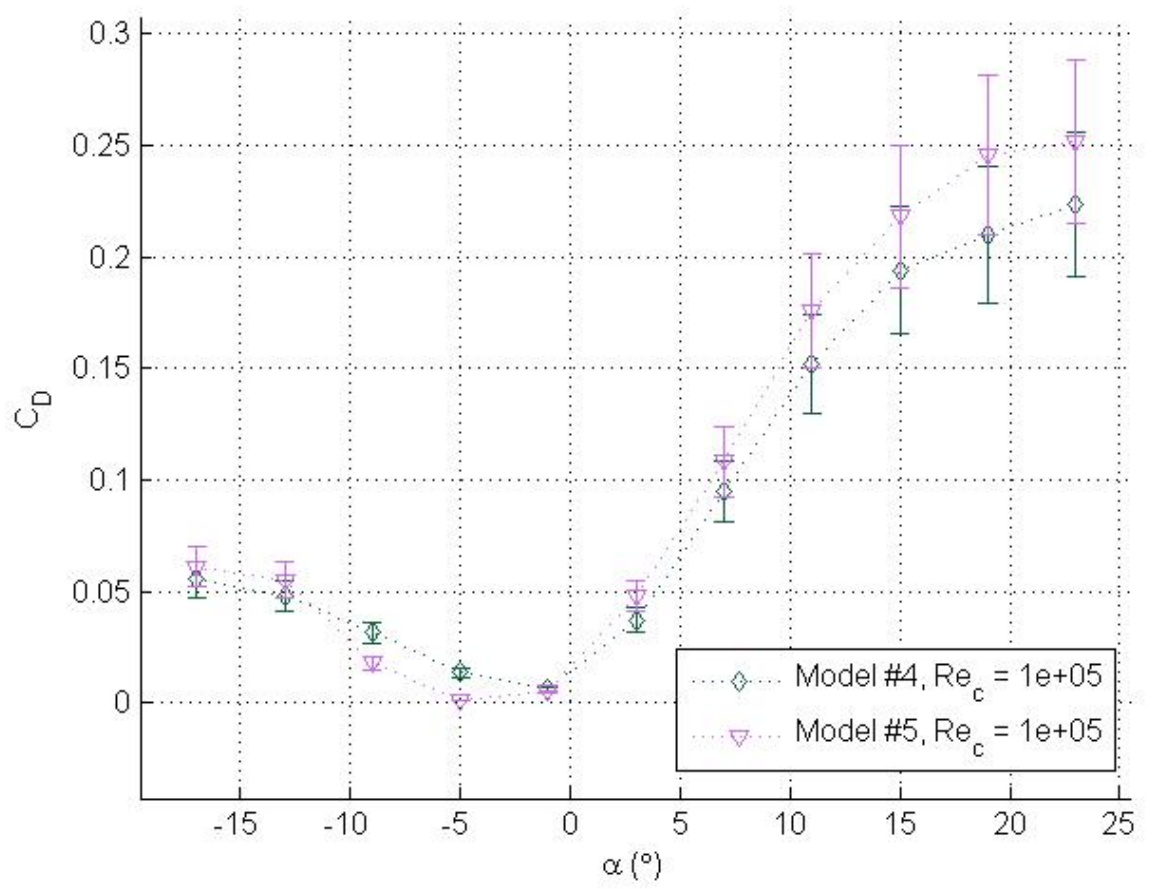

Figure 131: Comparison of the Differences in $C_{D}$ between the Cast and Plasti Dipped Wing Models at 100,000 Re

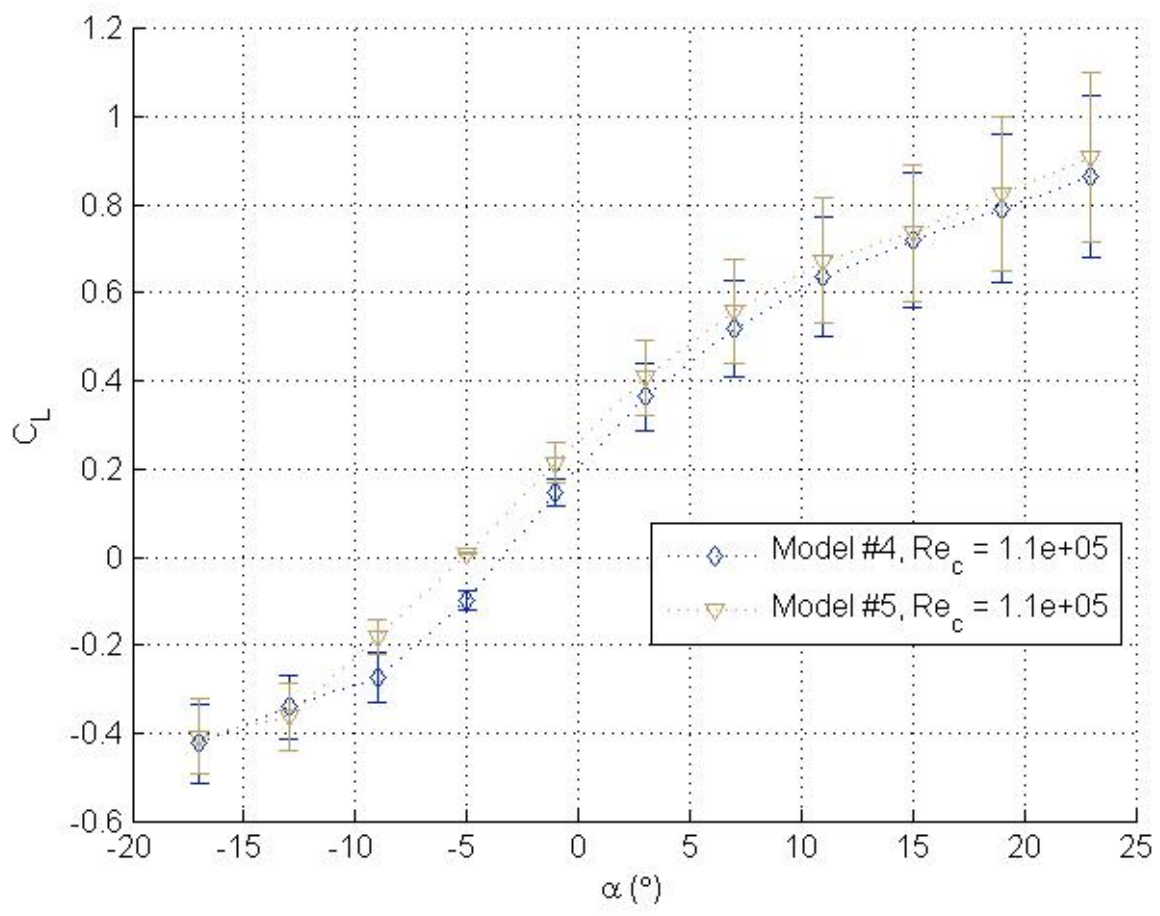

Figure 132: Comparison of the Differences in $C_{L}$ between the Cast and Plasti Dipped Wing Models at 110,000 Re 


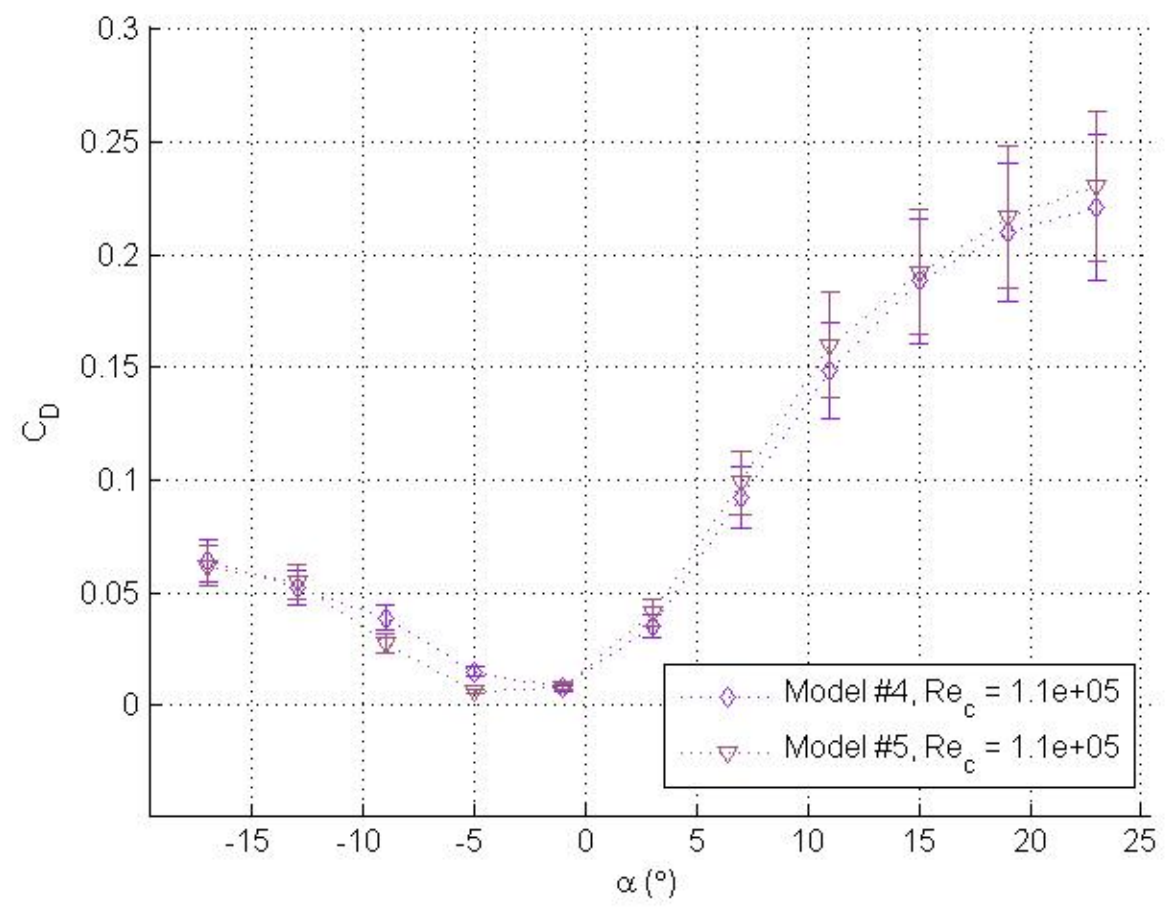

\section{Figure 133: Comparison of the Differences in $C_{D}$ between the Cast and Plasti Dipped Wing Models at 110,000 Re}

Figure 128 shows that at 93,000 Re, W5 showed higher $C_{L}$ values between $-5^{\circ}$ and $-1^{\circ} \alpha$. At this $R e$, W5 also showed lower $C_{D}$ values than $\mathrm{W} 4$ when $\alpha$ was between $-8^{\circ}$ and $-5^{\circ}$. When the $R e$ was increased to 104,000 , W5 showed increased $C_{L}$ values between $-8^{\circ}$ and $-1^{\circ} \alpha$ and decreased $C_{D}$ values when $\alpha$ was between $-8^{\circ}$ and $-5^{\circ}$ when compared to W4. This region of increased performance then shrank when the Re was increased to 109,000. At this $R e$, W5 showed a $C_{L}$ than W4 at $\alpha=-5$. W5 also showed a lower $C_{D}$ value at this $\alpha$. 


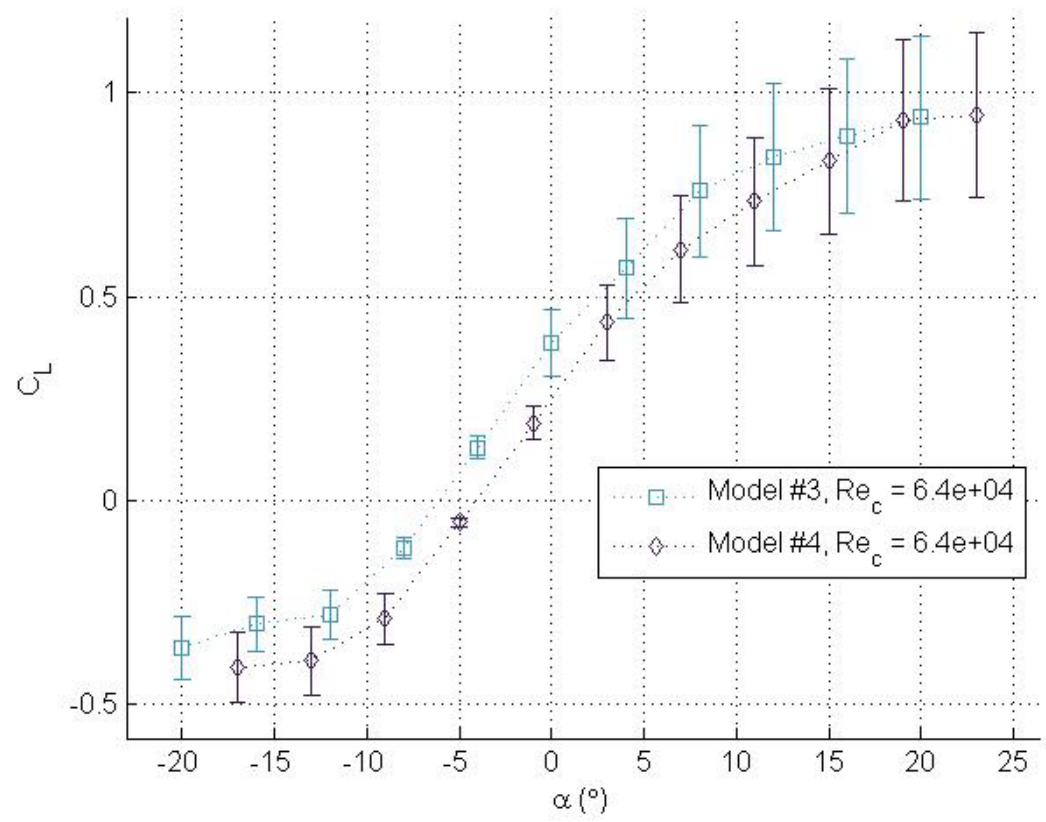

Figure 134: Comparison of the Differences in $C_{L}$ between the Polyurethane Coated and Cast Wing Models at 64,000 Re

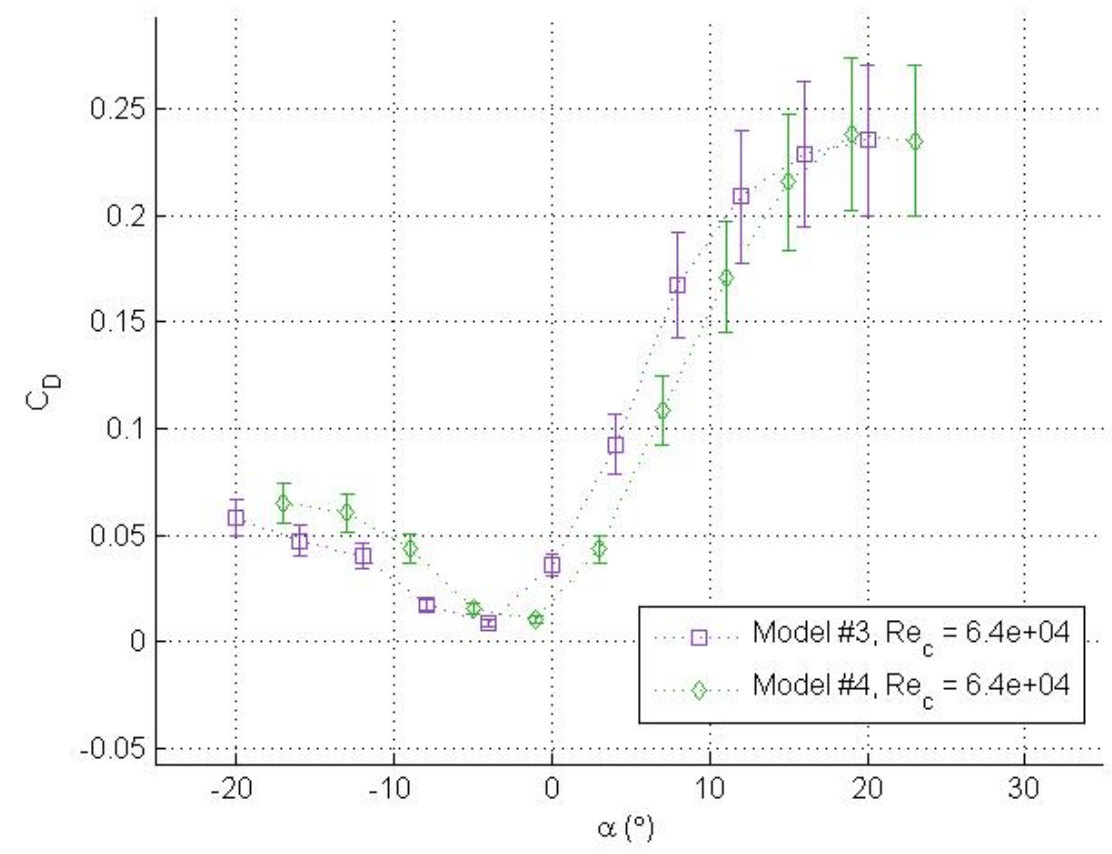

Figure 135: Comparison of the Differences in $C_{D}$ between the Polyurethane Coated and Cast Wing Models at 64,000 Re 


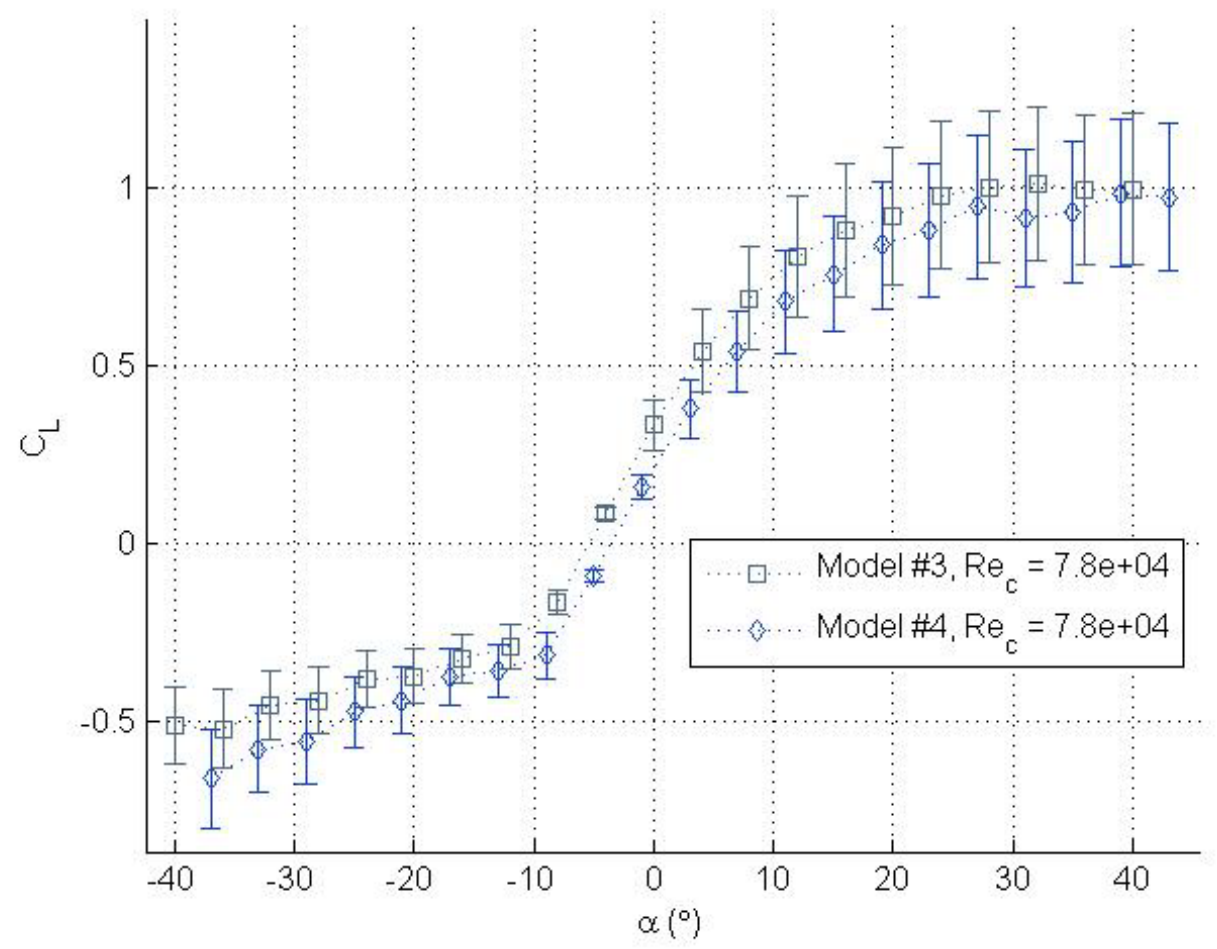

Figure 136: Comparison of the Differences in $C_{L}$ between the Polyurethane Coated and Cast Wing Models at 78,000 Re

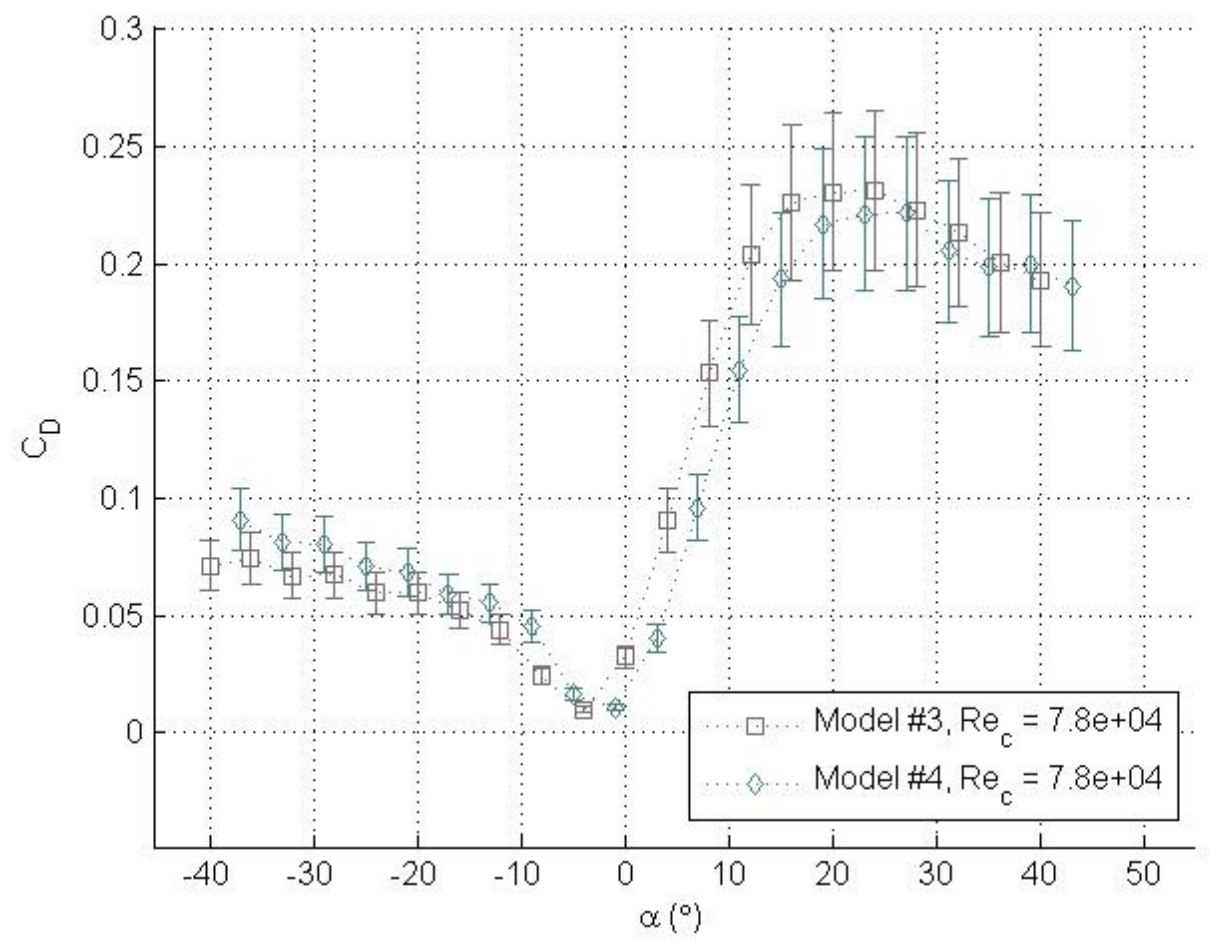

Figure 137: Comparison of the Differences in $C_{L}$ between the Polyurethane Coated and Cast Wing Models at 78,000 Re 
At $R e$ below 80,000 the two wings performed similarly. W3 showed increased $C_{L}$ values at $\alpha=-8^{\circ}$ and $\alpha=-4^{\circ}$. W3 also showed decreased $C_{D}$ values at $\alpha=-8^{\circ}$. However, W4 showed lower $C_{D}$ values when $\alpha$ was between $-1^{\circ}$ and $3^{\circ}$.

Figure 138 shows that the performance differences similar to those shown in Figure 134 are also present when the $R e$ is 93,000. The higher $C_{L}$ values of W3 disappear at $R e$ above 93,000. However, the lower $C_{D}$ values of $\mathrm{W} 4$ between $-1^{\circ}$ and $3^{\circ}$ remain when $R e$ is 93,000 . Above 93,000 Re, no differences in $C_{L}$ are present between $\mathrm{W} 3$ and $\mathrm{W} 4$, but W4 shows lower $C_{D}$ values at $\alpha=-1^{\circ}$.

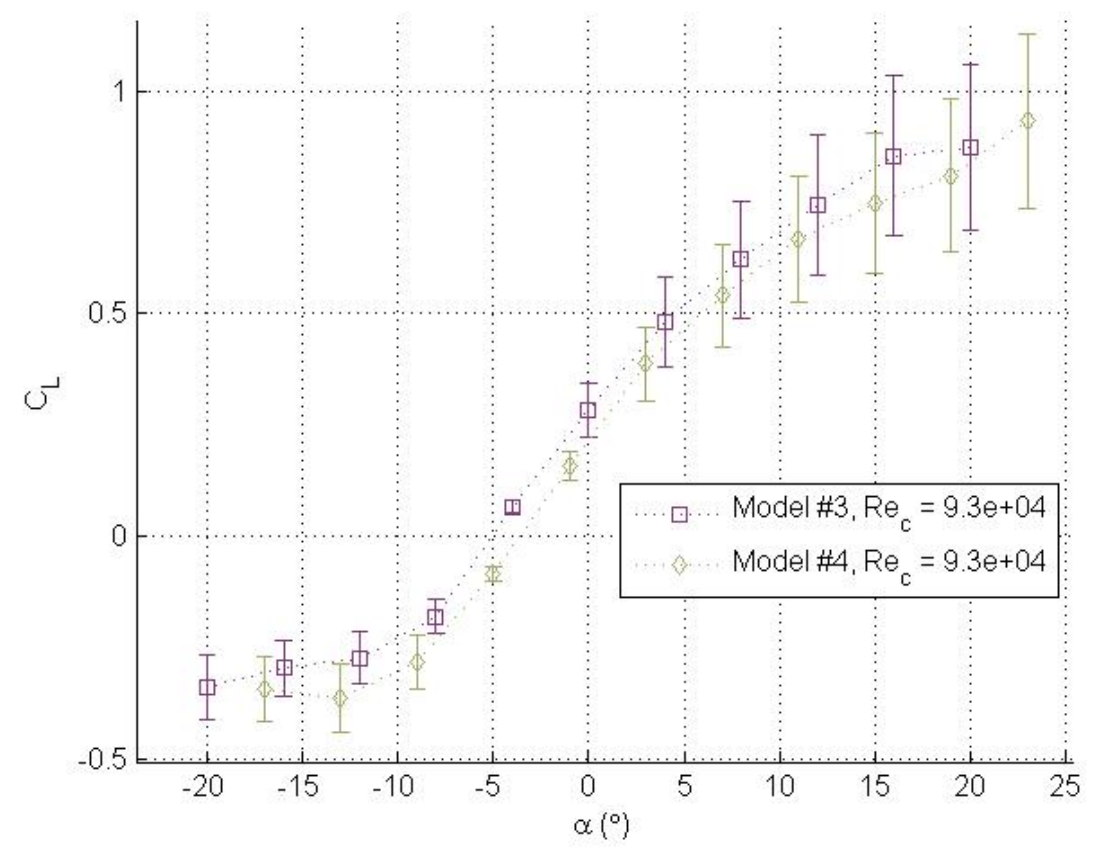

Figure 138: Comparison of the Differences in $C_{L}$ between the Polyurethane Coated and Cast Wing Models at 93,000 Re 


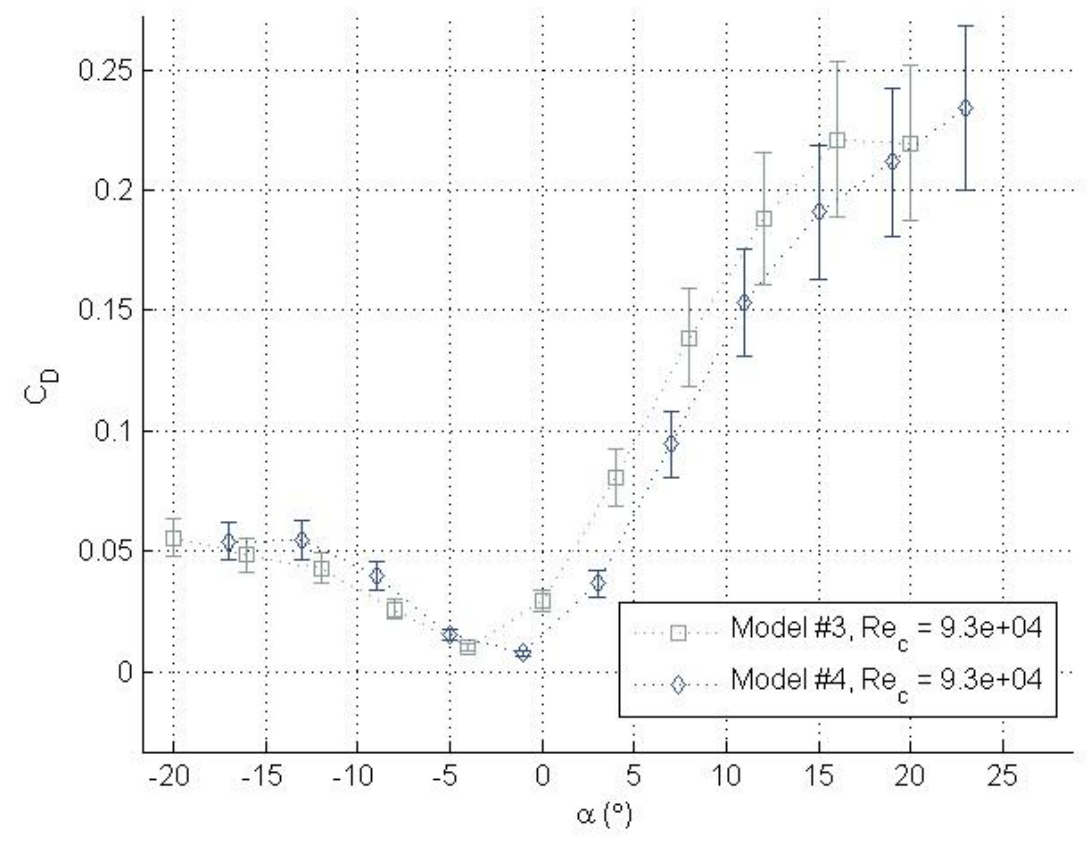

Figure 139: Comparison of the Differences in $C_{D}$ between the Polyurethane Coated and Cast Wing Models at 93,000 Re

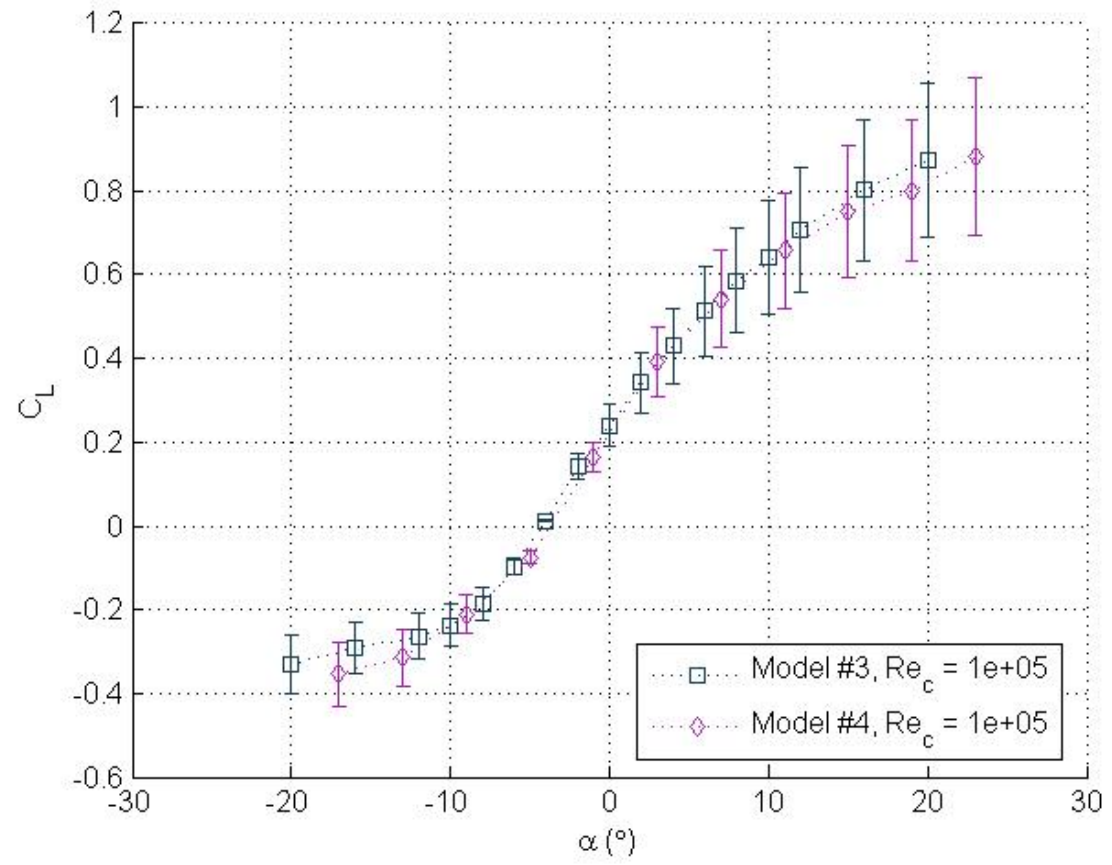

Figure 140: Comparison of the Differences in $C_{L}$ between the Polyurethane Coated and Cast Wing Models at 100,000 Re 


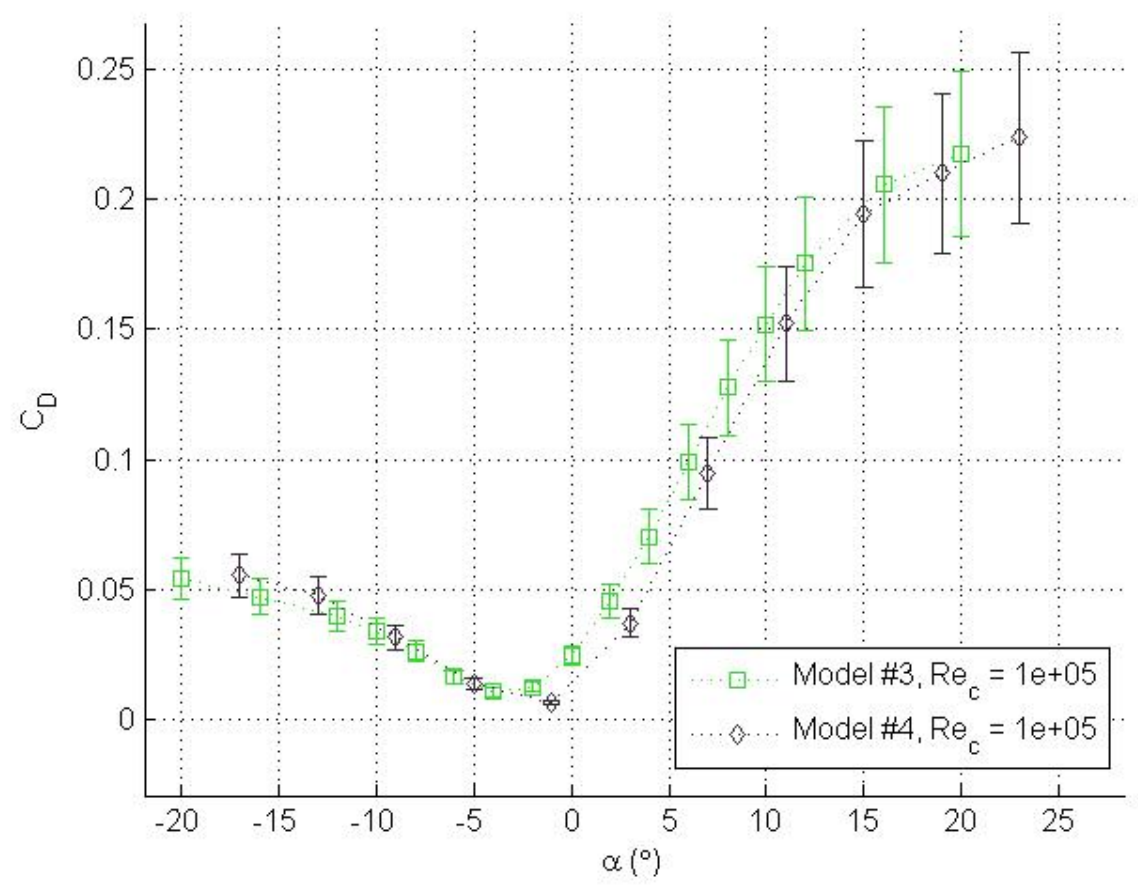

Figure 141: Comparison of the Differences in $C_{D}$ between the Polyurethane Coated and Cast Wing Models at 100,000 Re

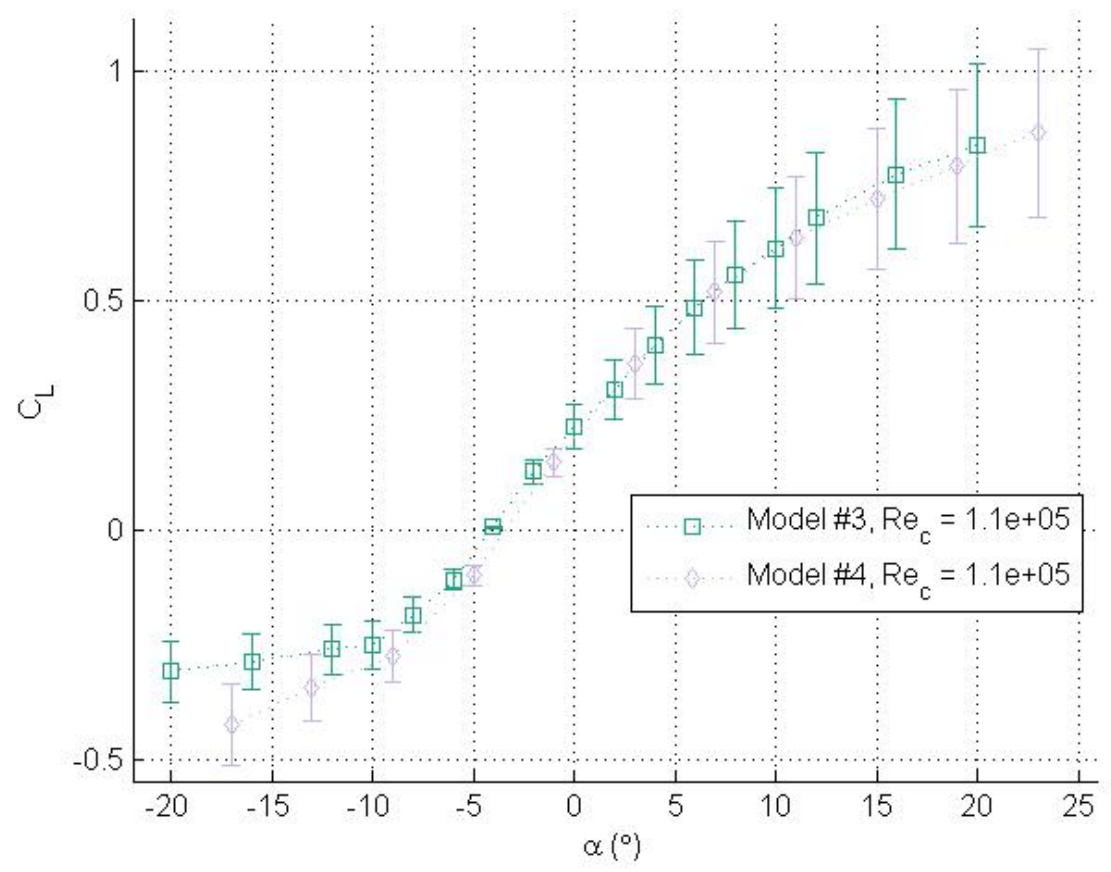

Figure 142: Comparison of the Differences in $C_{L}$ between the Polyurethane Coated and Cast Wing Models at 110,000 Re 


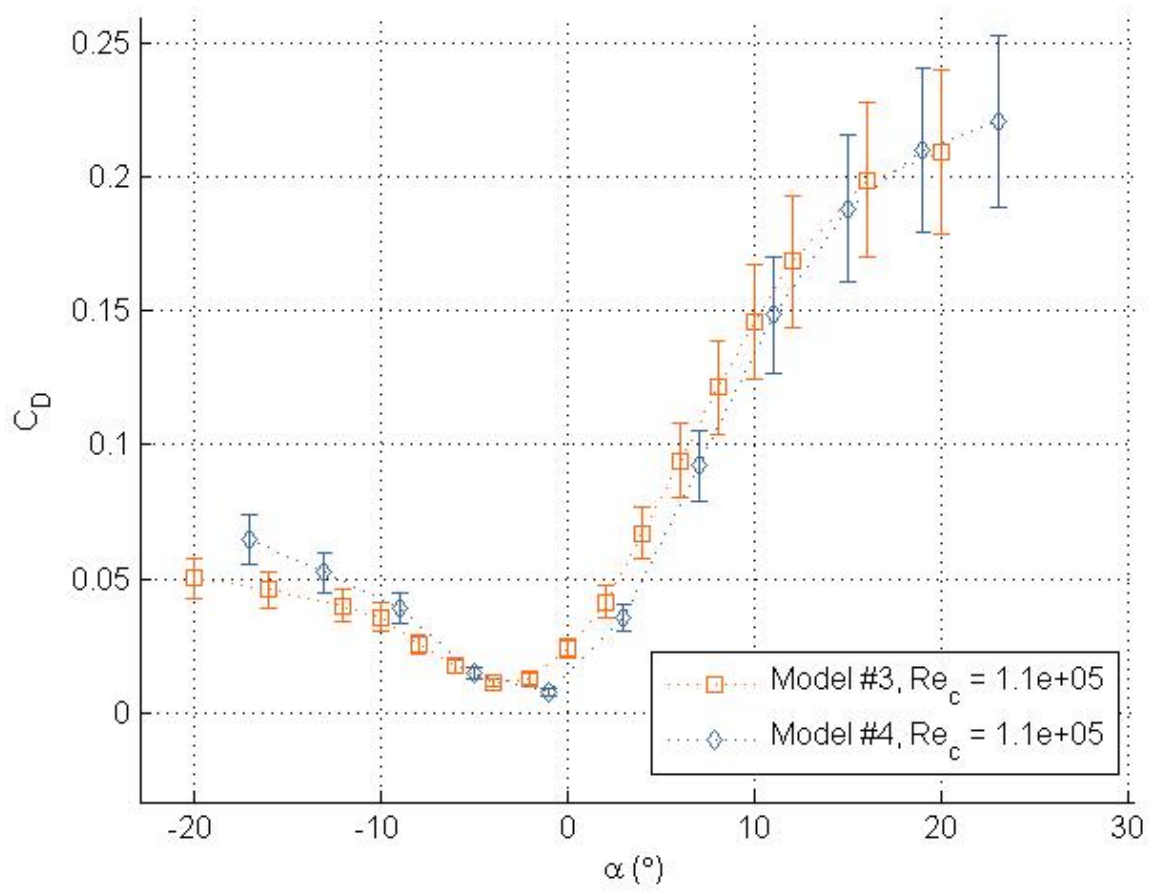

Figure 143: Comparison of the Differences in $C_{D}$ between the Polyurethane Coated and Cast Wing Models at 110,000 Re

Polyurethane Coated Wing Model vs. Plasti Dipped Wing Model

The main difference noticed between the performance of W3 and W5 in Figure 144 was the $C_{D}$ curve. W5 showed a lower $C_{D}$ value than W3 at $\alpha=-1^{\circ}$ for each curve when the $\operatorname{Re}$ was below 80,000. As the Re increased from 64,000, the $C_{L}$ and $C_{D}$ curves of the two wings began to converge. 


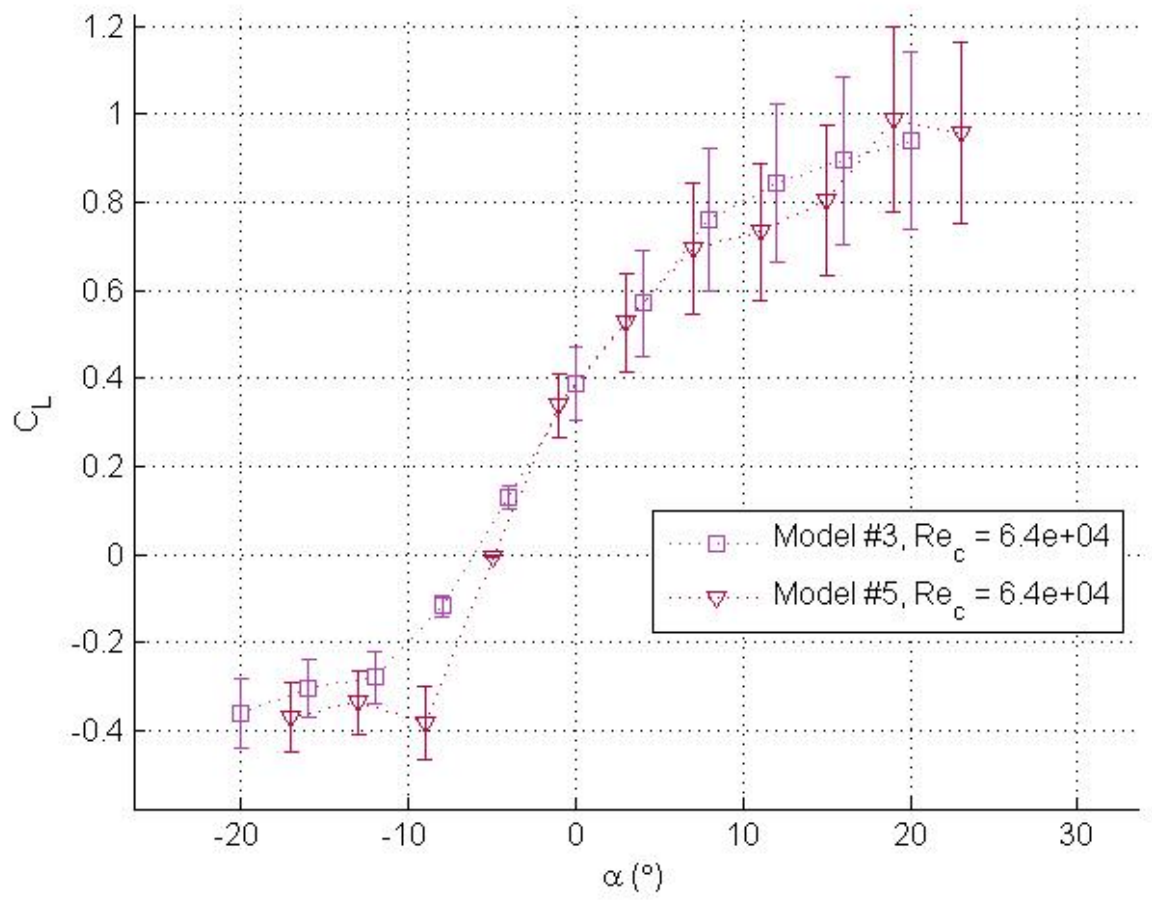

Figure 144: Comparison of the Differences in $C_{L}$ between the Polyurethane Coated and Plasti Dipped Wing Models at 64,000 Re

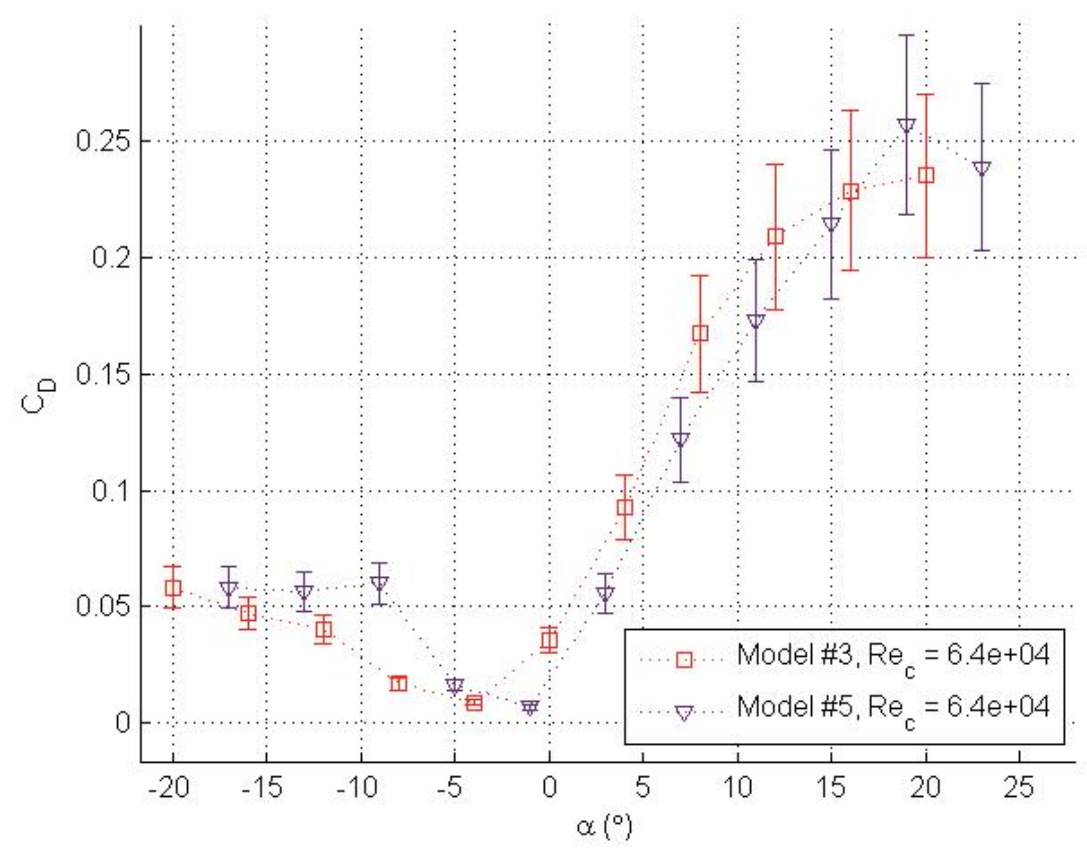

Figure 145: Comparison of the Differences in $C_{D}$ between the Polyurethane Coated and Plasti Dipped Wing Models at 64,000 Re 


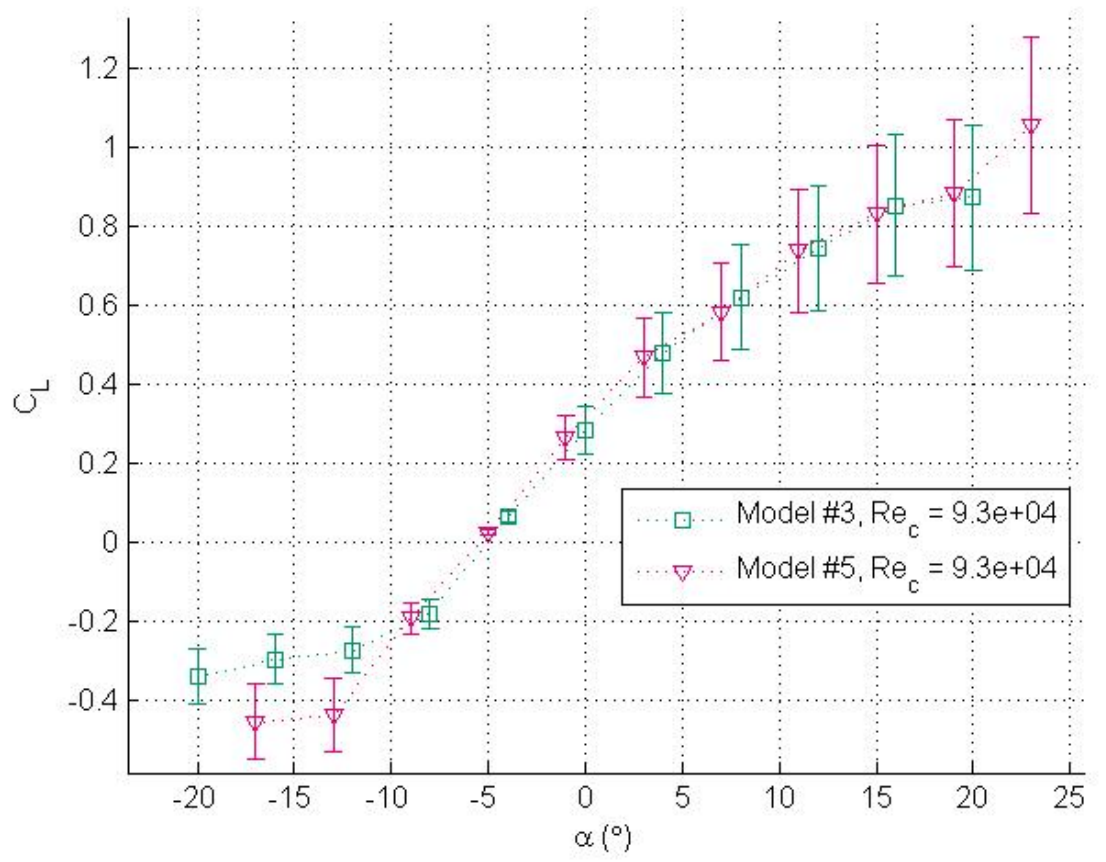

Figure 146: Comparison of the Differences in $C_{L}$ between the Polyurethane Coated and Plasti Dipped Wing Models at 93,000 Re

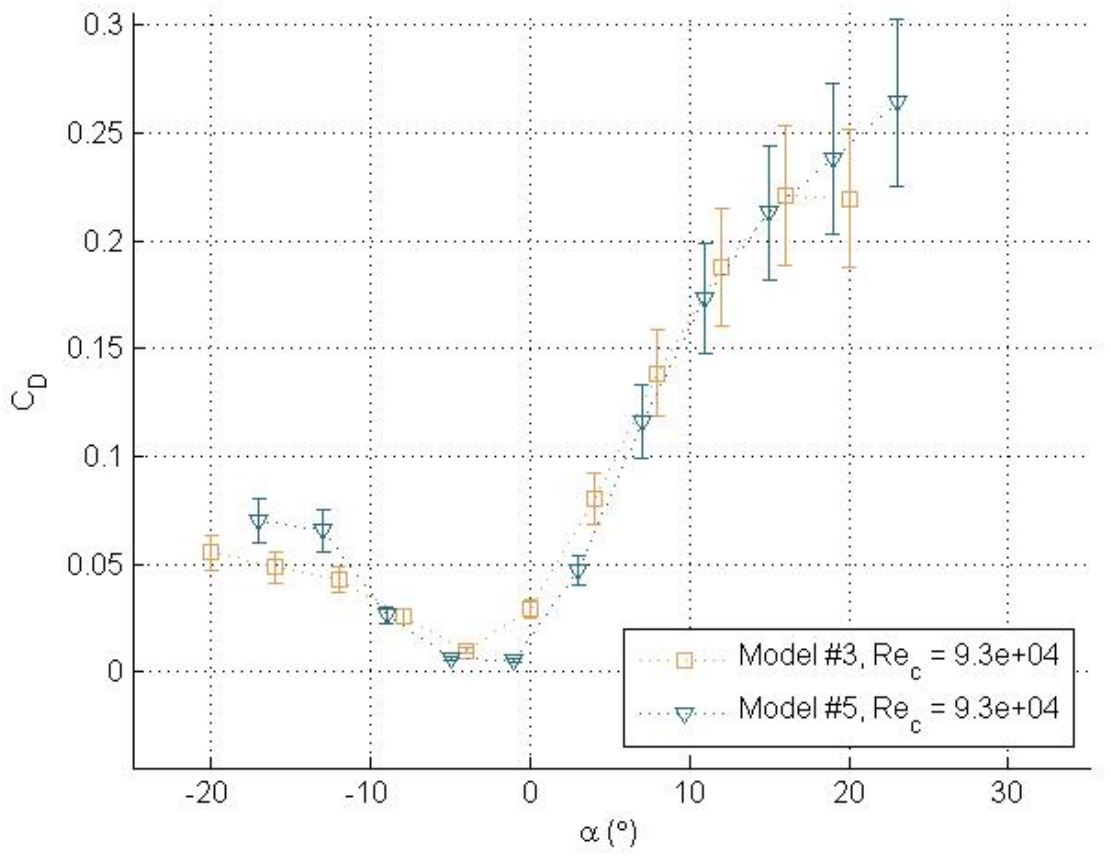

Figure 147: Comparison of the Differences in $C_{D}$ between the Polyurethane Coated and Plasti Dipped Wing Models at 93,000 Re 


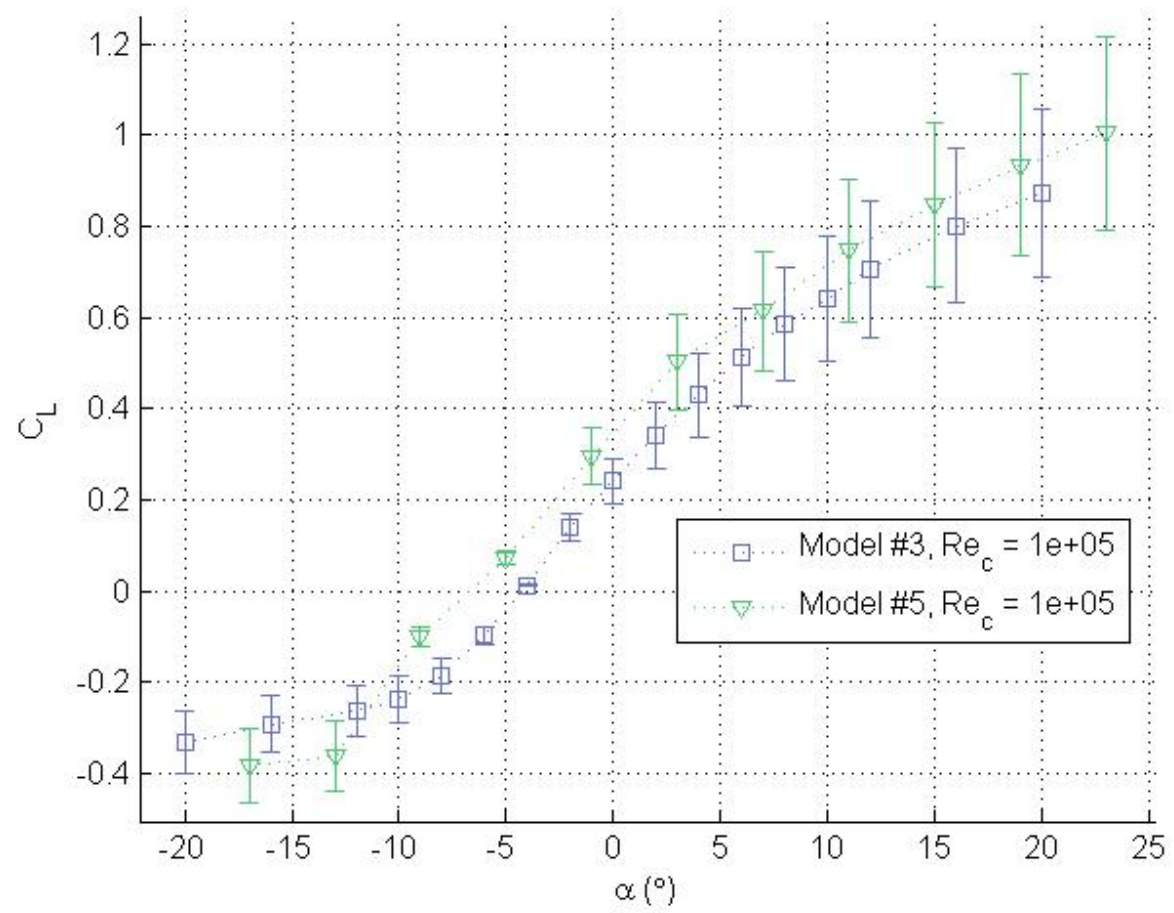

Figure 148: Comparison of the Differences in $C_{L}$ between the Polyurethane Coated and Plasti Dipped Wing Models at 100,000 Re

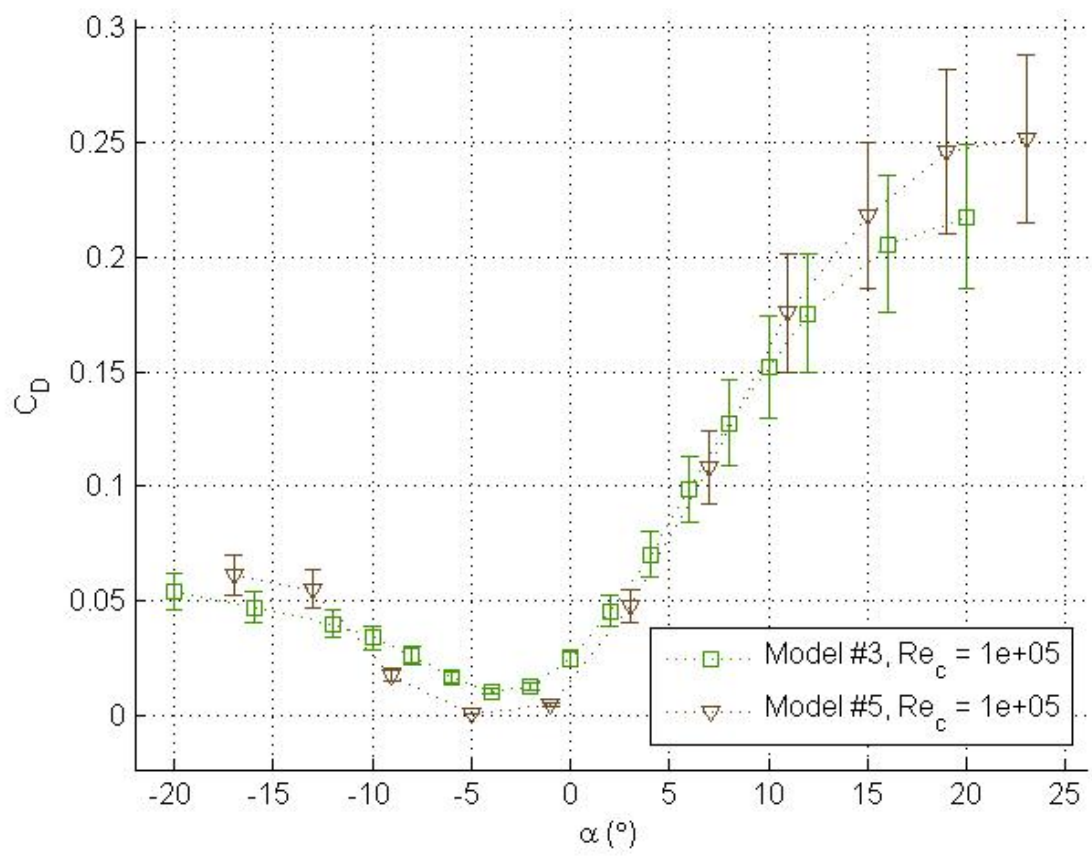

Figure 149: Comparison of the Differences in $C_{D}$ between the Polyurethane Coated and Plasti Dipped Wing Models at 100,000 Re 


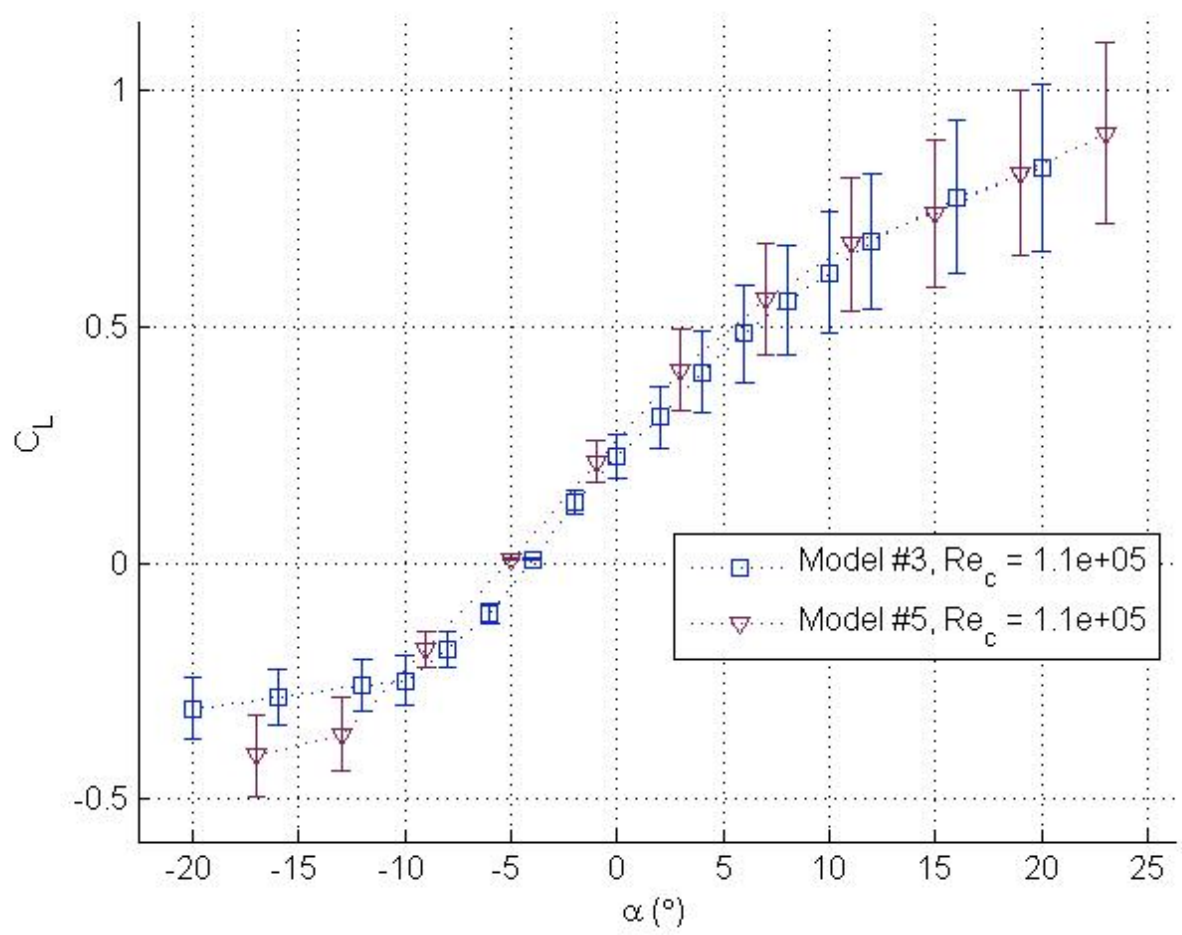

Figure 150: Comparison of the Differences in $C_{L}$ between the Polyurethane Coated and Plasti Dipped Wing Models at 110,000 Re

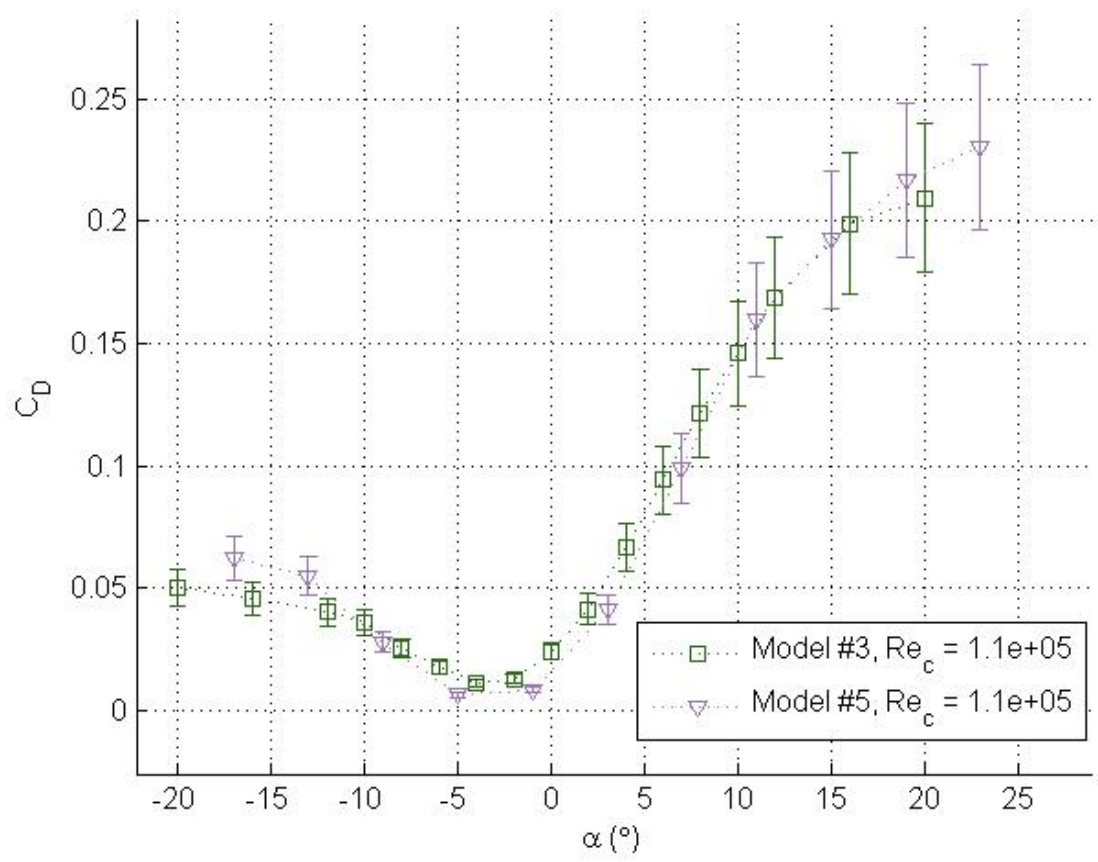

Figure 151: Comparison of the Differences in $C_{D}$ between the Polyurethane Coated and Plasti Dipped Wing Models at 110,000 Re 
As the $R e$ was increased above 90,000 the range of lower $C_{D}$ values for W5 began to increase. By the time Re was $95,000, C_{D}$ values for $\mathrm{W} 5$ were lower than $\mathrm{W} 3$ from $-5^{\circ}$ to $3^{\circ} \alpha$. Above 95,000 the range of $\alpha$ at which this occurred began to decrease and shift left to include $\alpha$ from $-10^{\circ}$ to $-1^{\circ}$.

Differences in $C_{L}$ between W3 and W5 were inconsistent across the tested Re range.

However, W5 exhibited lower $C_{D}$ and higher $C_{L}$ values than W3 when $\alpha$ was between $-8^{\circ}$ and $-1^{\circ}$ and $R e$ was 104,000 . This also occurred at $\alpha=-5^{\circ}$ when $R e$ was 109,000 . 


\subsubsection{Overall Comparison}

Figure 152 shows that when compared to all the other wing models, W2 showed the highest $C_{L}$ values. W1 and W3 also performed very similarly at lower angles of attack when $R e$ was 64,000 . This changed as the angle of attack increased. Above $3^{\circ} \alpha$, the $C_{L}$ curve of W1 shows lower $C_{L}$ values.

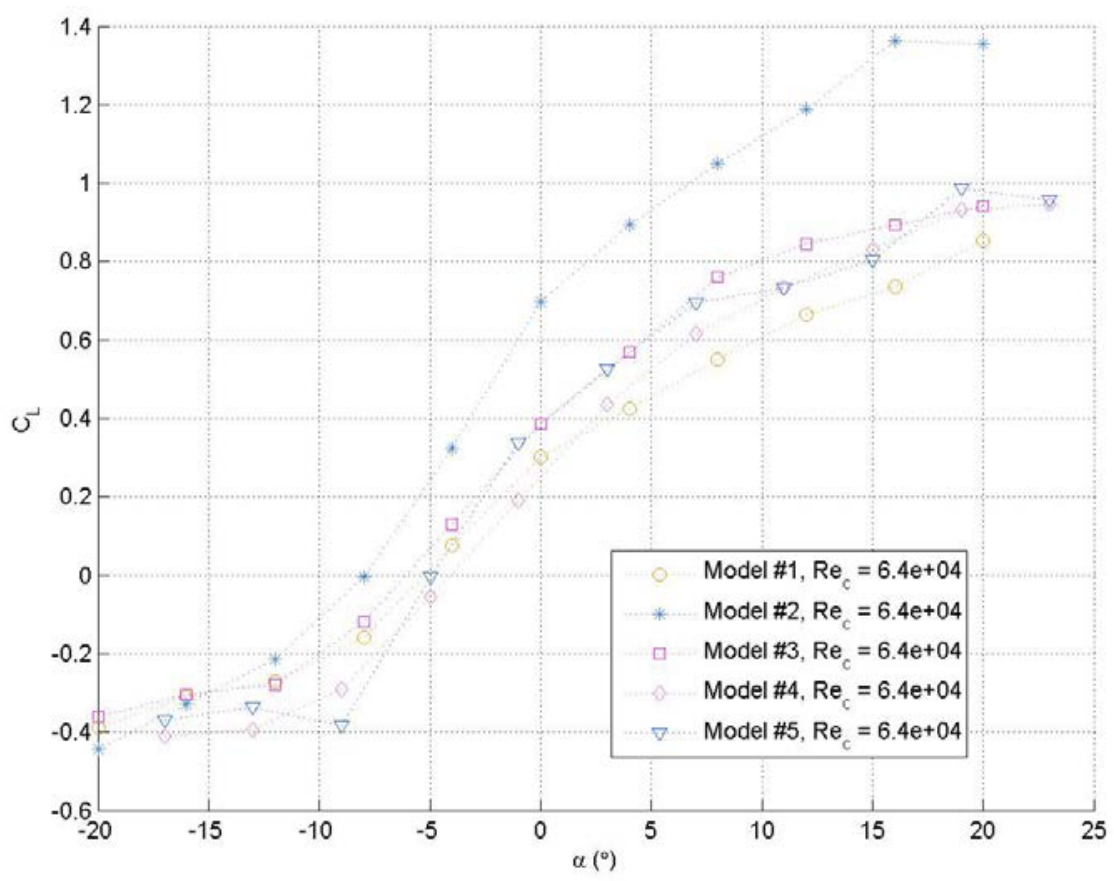

Figure 152: A Comparison of the $C_{L}$ Curve for each Model at 64,000 Re

W2 had the highest $C_{D}$ values as seen in Figure 153. W2 also had the most predictable curve. The $C_{D}$ curve for the other models did not show the same smooth transitions. 


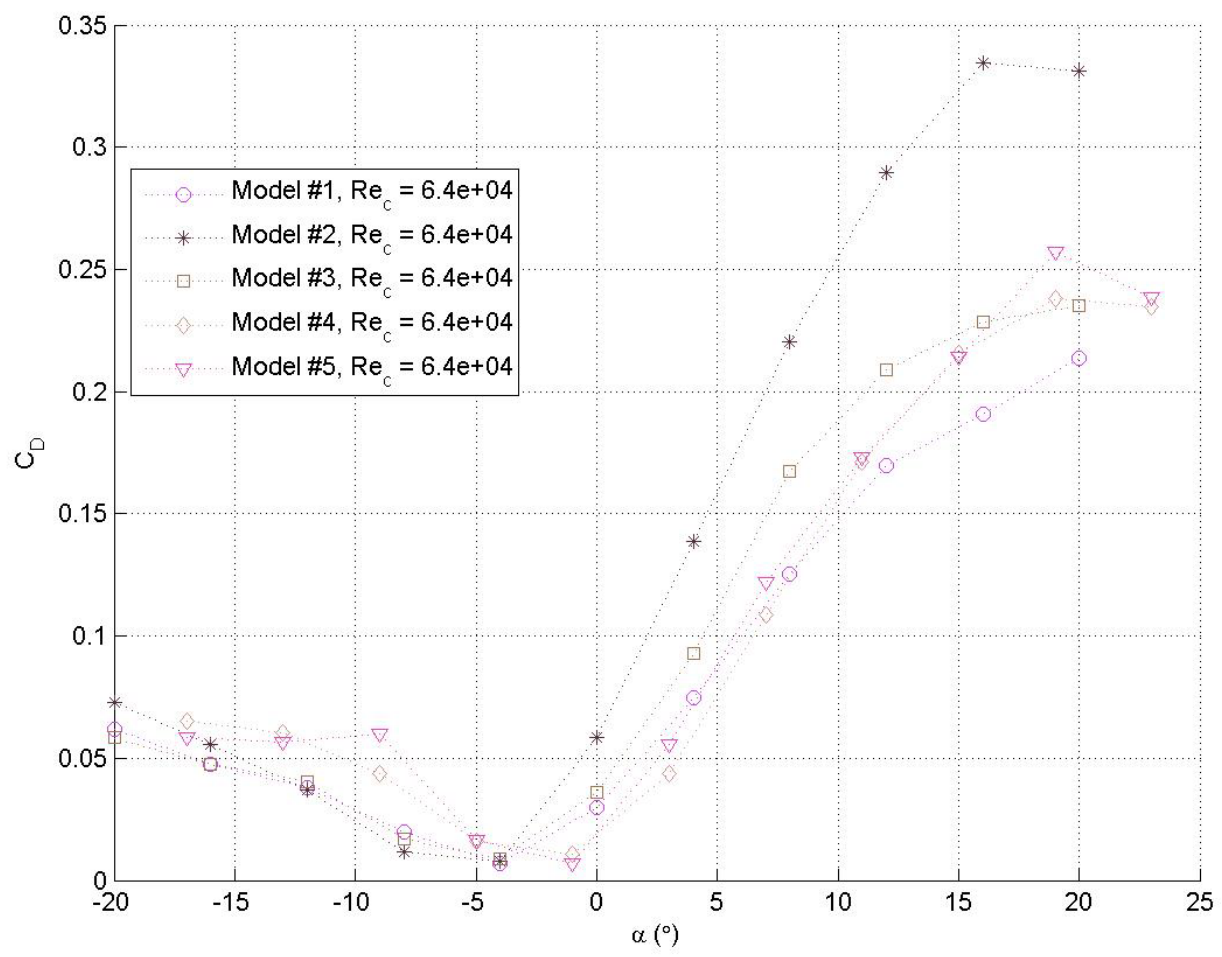

Figure 153: A Comparison of the $C_{D}$ Curve for each Model at 64,000 Re

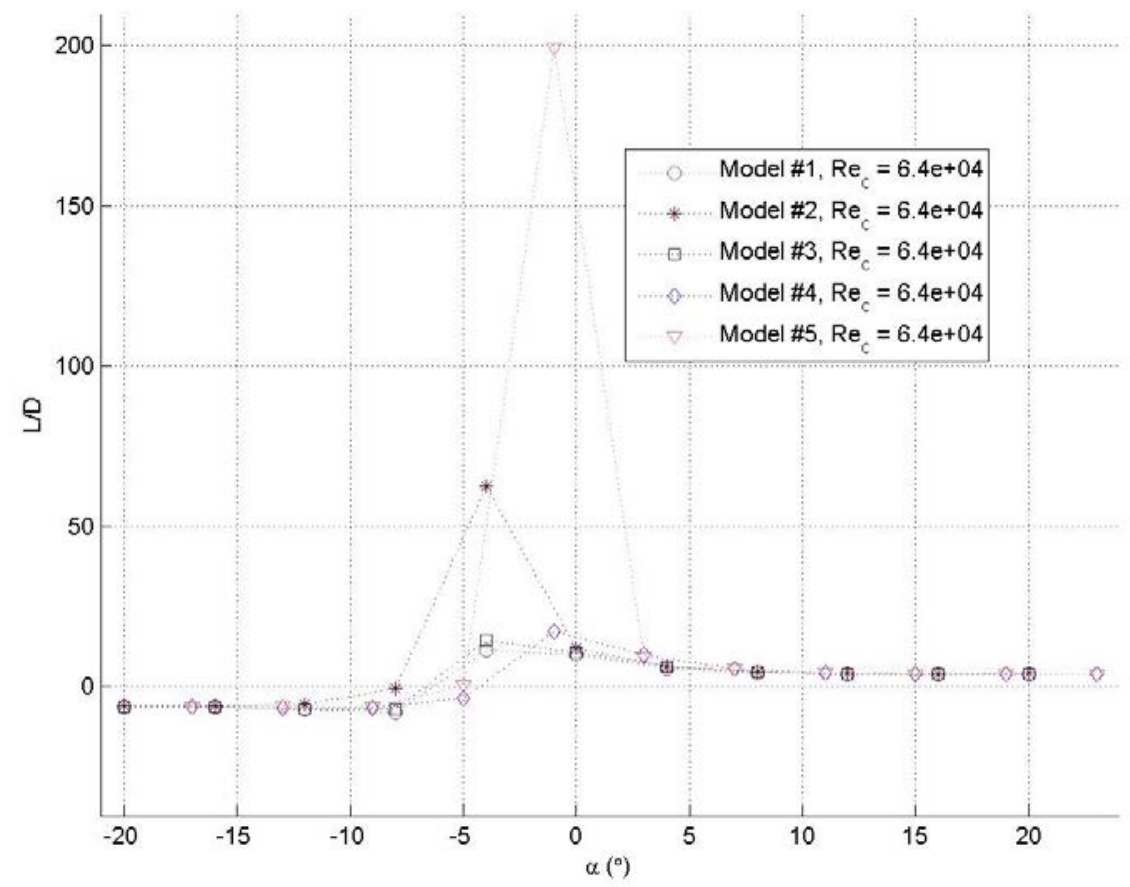

Figure 154: A Comparison of the $L / D$ Curve for each Model at 64,000 Re 
Figure 155 shows that W2 continued to outperform the other models even at the highest Re tested. W1 performed the worst out of all the models at $\alpha$ above $3^{\circ}$ for this $R e$. This figure also shows that W3 and W4 performed similarly above $-10^{\circ} \alpha$.

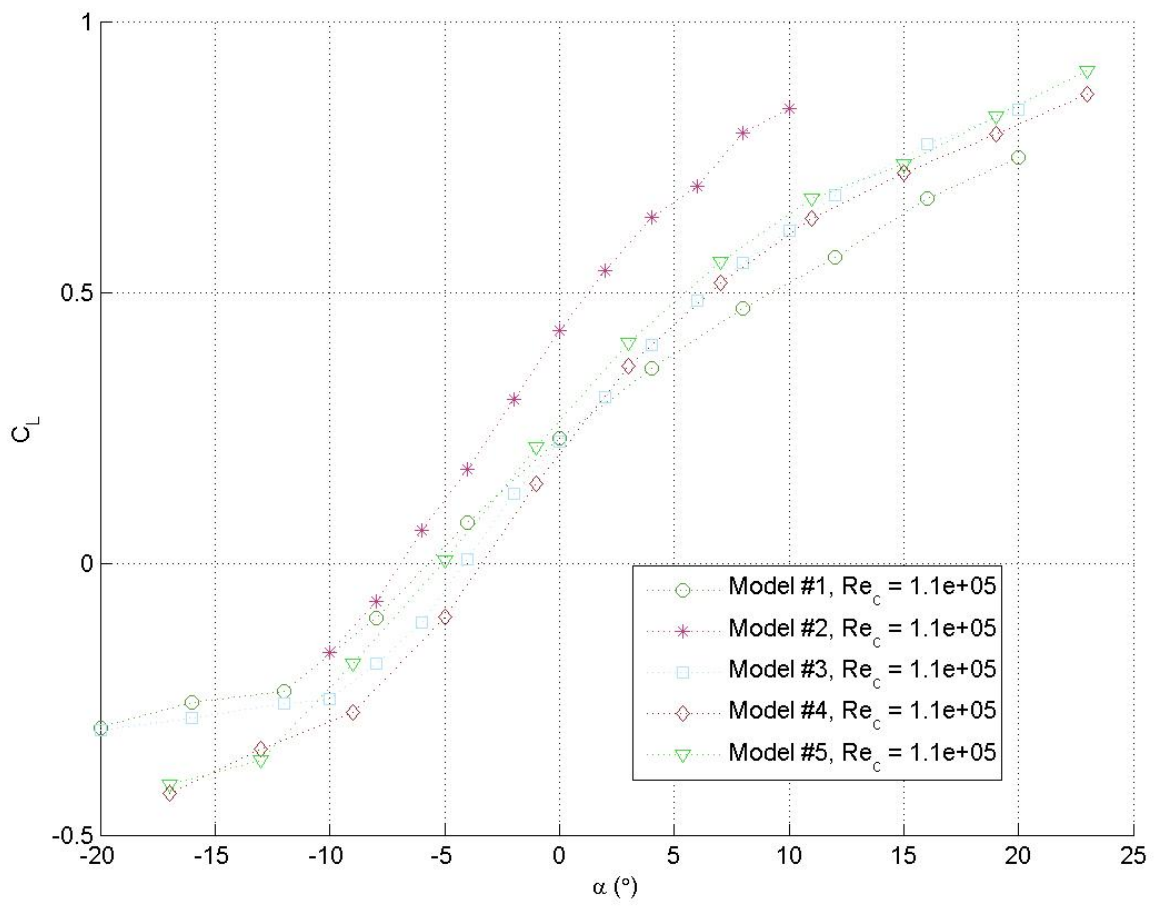

Figure 155: A Comparison of the $C_{L}$ Curve for each Model at 110,000 Re

At $110,000 R e, \mathrm{~W} 2$ also showed the highest and lowest $C_{D}$ value between $-10^{\circ}$ and $10^{\circ} \alpha$. Overall, W1 showed the lowest $C_{D}$ values in Figure 156 . W3, W4, and W5 showed similar $C_{D}$ values. However, W5 did show noticeably lower $C_{D}$ values when $\alpha$ was between $-5^{\circ}$ and $-2^{\circ}$. 


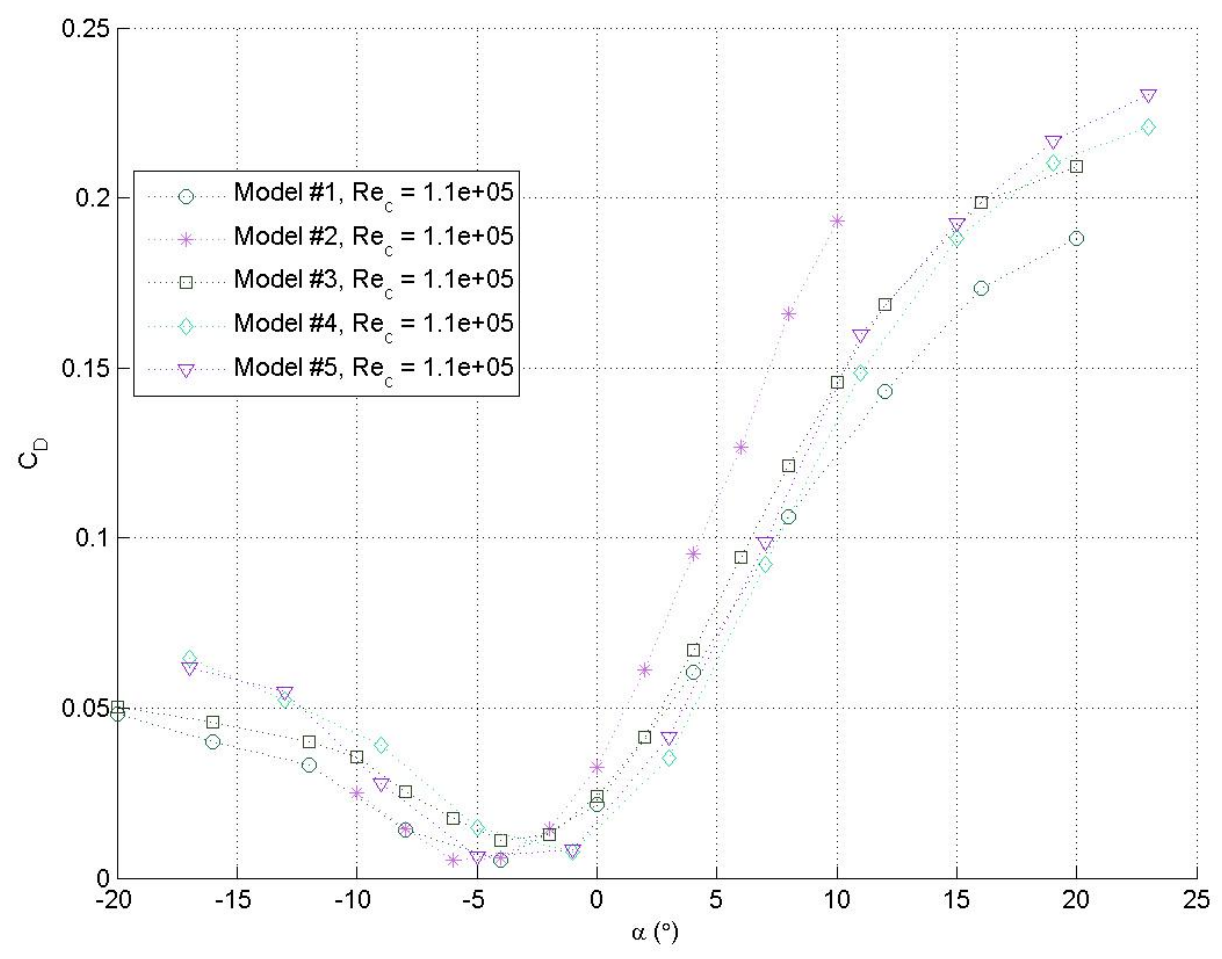

Figure 156: A Comparison of the $C_{D}$ Curve for each Model at 110,000 Re

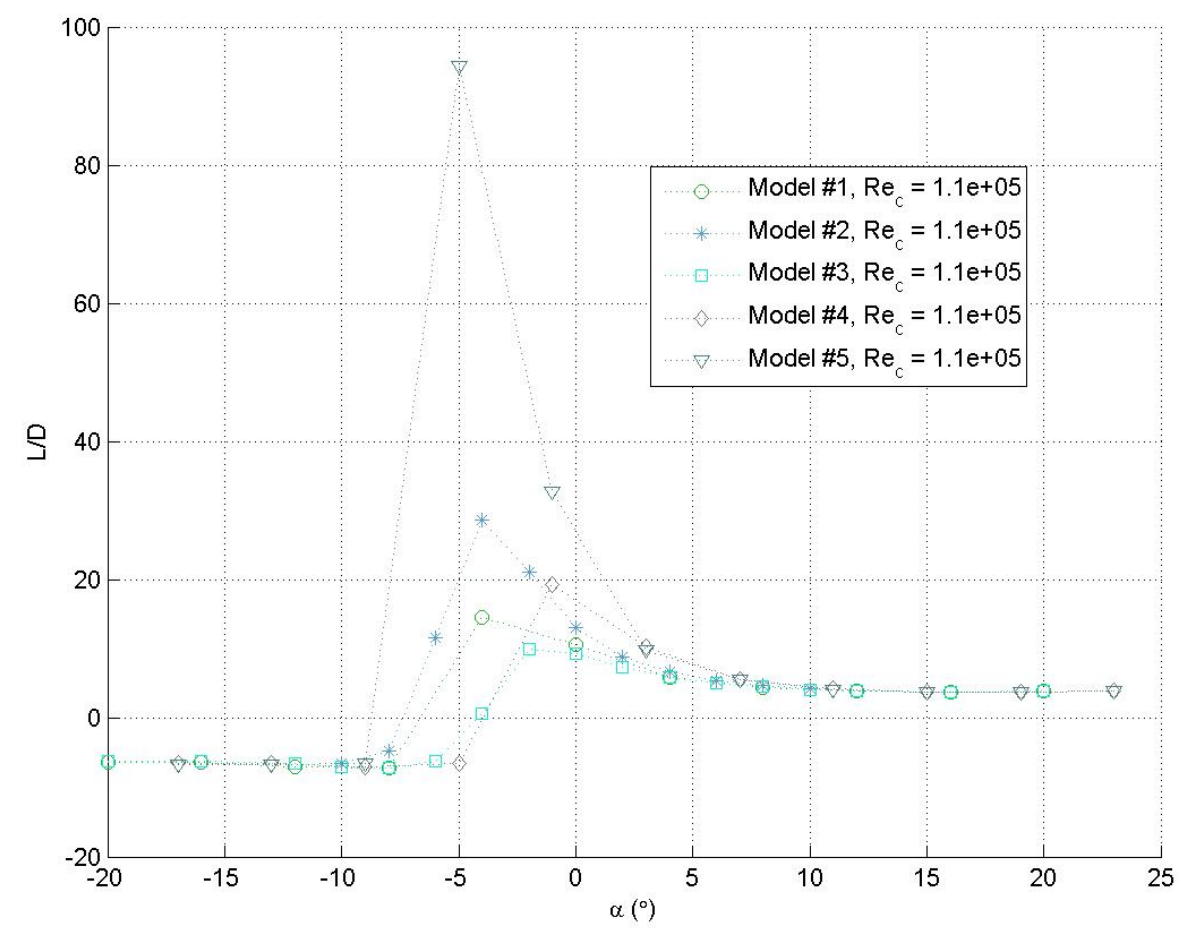

Figure 157: A Comparison of the $L / D$ Curve for each Model at 110,000 $R e$ 


\section{Chapter 6: Conclusions}

\section{Feather Motion}

Of all the wing models, W1 and W2 exhibited the smallest changes in feather motion and surface geometry. By comparing the aerodynamic performance of W1 and W2, it was noticed that W2 had a clear performance advantage. Through further investigation, it was found that W2 showed favorable aerodynamic performance over W1 when the covert or secondary covert feathers of W2 showed increased frequencies and lower amplitudes.

Although W3 exhibited reduced feather motion overall and W4 had no feather motion, their performance was similar to $\mathrm{W} 1$ and $\mathrm{W} 2$ at negative $\alpha$ at which the covert and secondary coverts of the wings were not active. Yet, once covert and secondary covert feathers of W1 and W2 began to move, the performance of W1 and W2 were increased when compared to W3 and W4.

However, these benefits were only observed at $\alpha$ where both the covert and secondary covert feathers exhibited some motion. For example, at $\alpha$ where secondary coverts of W2 showed higher frequencies than those of W1, no performance benefit was observed. Yet, if both feather groups exhibited motion, performance benefits were found. These effects were also exhibited by W1, when compared to W3 and when W2 was compared to W4. This supports that higher levels of covert and secondary covert feather frequency and lower amplitude, increased the performance of the wing. Performance effects related to primary and secondary feather motion were not found.

Applying hair spray to individual feathers was found to decrease the resonant frequency of the feathers. Hair spray was also found to cause the amplitude of covert and secondary covert 
feathers on the hair sprayed wing to decrease. The application of hairspray therefore, caused feather motion to occur much closer to the wing itself. These conclusions correspond with the effects of dynamic surface roughness using high frequency and low amplitude oscillations. "Low amplitude, high frequency surface actuation, in the form of vibrating surface roughness, can be used to accelerate the laminar boundary layer, thereby suppressing certain forms of laminar separation and producing an attached flow in situations that would otherwise be separated" [28]. It is believed that the decreased amplitude and increased frequency of the covert feathers on W2 have a similar effect.

\section{Surface Geometry}

W4 and W5 had the same macroscopic shape and no moving feathers. When these two models were compared, W5 was found to perform better than W4 overall. The primary differences in surface geometry between W4 and W5 were found to be the $58 \%$ increase in both barbule length and angle as seen in Table 5 . However, the 58\% change for both of the features was not believed to be a coincidence. W5 also showed a 9.5 $\mu \mathrm{m}$ average decrease in surface feature depth. Therefore, the 58\% change in barbule length and angle was attributed to taking the measurements of W5 on a plane $9.5 \mu$ higher than W4.

In order to better understand the effects of differences in surface geometry, the aerodynamic performance of W3 was compared to W4. W3 was chosen for this comparison since it showed the least amount of feather movement. Although Table 4 shows a small amount of primary and secondary feather motion on W3, the performance advantages of W3 did not show a direct relation to the feather movement. 
The figures provided on pages 131 through 140 show that W4 did not outperform W3 in any case. Figure 53 shows the greatest difference in the surface geometry of the two wings to be the depth of the surface features. The average depth of the surface features of W4 was found to be $70.5 \mu$ deeper than W3. The changes in surface geometry provided in Table 5 show that although the geometry of almost every surface feature was different between the two wings, only the barbule length and depth of surface features showed corresponding changes.

When the $C_{L}$ and $C_{D}$ curves of W3 and W5 were compared, W5 consistently showed lower $C_{D}$ values. When the differences in the surface geometry were examined, surface depth was only consistent change between W3, W4, and W5. The fact that differences in performance were observed between W3 and W4 as well as W3 and W5 supports that surface geometry has an effect on the performance of a wing. However, when the minimal performance differences observed between W3 and W4 are considered, it can be determined that although the depth of the surface features can affect the performance of the wing, these performance effects only occur within a limited range of surface feature depth.

\section{Chapter 7: Recommendations}

Applying synthetic structures similar to feathers to the wing surface of small UAV operating at low Reynolds numbers could improve the performance of that UAV. Increasing the frequency of the feathers located in the same area of the UAV's wing as covert and secondary covert feathers could further increase the performance of the UAV. This could be done by adjusting the material, thickness, and geometry of the feathers to increase their frequency.

Replication of the surface geometry of feathers on the synthetic feathers of a UAV could also improve the performance of the UAV by reducing the UAV's $C_{D}$. However, replicating the 
fine surface features of a feather is not believed to have a positive effect on the performance of a wing. Therefore, tooling and machining cost can be reduced by allowing lower tolerances. 


\section{Works Cited}

[1] J. Wang and J. Schluter, "Low Reynolds Number Application of Feather Inspired Passive High Lift Device on Finite Wing," AIAA, 2013.

[2] U. L. Norberg, "Structure, Form, and Function of Flight in Engineering and the Living World," Journal of Morphology, pp. 52-81, 2002.

[3] L. De Vinci and E. MacCurdy, Leonardo Da Vinci's Note-Books, New York: Empire State Book, 1923.

[4] J. M. Benyus, Biomimicry: Innovation Inspired by Nature, New York: Morrow, 1997.

[5] R. A. Kroeger, H. D. Grushka and T. C. Helvey, "Section 1: Introduction," in Low Speed Aerodynamics for Ultra-quiet Flight, Springfield, VA, National Technical Information Service, 1972.

[6] "Leonardo Da Vinc's Dream of Flying," LeonardoDaVinci.net, [Online]. Available: http://www.leonardodavinci.net/flyingmachine.jsp. [Accessed July 2015].

[7] "Otto Lilienthal," Encyclopedia.com, 01 January 2004. [Online]. Available: http://www.encyclopedia.com/topic/Otto_Lilienthal.aspx. [Accessed 28 May 2015].

[8] M. Dickinson, "Bionics: Biological Insight into Mechanical Design," in National Academy of Sciences of the United States of America, 1999.

[9] "1903 Wright Flyer," Smithsonian National Air and Space Museum, [Online]. Available: http://airandspace.si.edu/collections/artifact.cfm?object=nasm_A19610048000. [Accessed 28 May 2015].

[10] A. C. Carruthers, G. K. Taylor, S. M. Walker and A. L. Thomas, "Use and Function of a Leading Edge Flap on the Wings of Eagles," AIAA, 2007.

[11] E. F. Blick, "The Aerodynamics of Birds," AIAA Student Journal, 1976.

[12] J. J. Videler, Avian Flight, Oxford: Oxford UP, 2005.

[13] J. J. Videler, E. J. Stamhuis and G. Povel, "Leading-Edge Vortex Lifts Swift," Science, pp. 1960-1962, 2004.

[14] D. Blood, V. P. Studdert and C. C. Saunders, Comprehensive Veterinary Dictionary, Edinbrugh: Elsevier Saunders, 2007.

[15] D. White, "Parrot Anatomy," Parrot Fun Zone, [Online]. Available: http://parrotfunzone.com/learn-about-parrots/parrot-anatomy/external-anatomy. [Accessed 21 Febuary 2016].

[16] V. A. Tucker, "GLIDING BIRDS: REDUCTION OF INDUCED DRAG BY WING TIP SLOTS BETWEEN THE PRIMARY FEATHERS," Experimental Biology, pp. 285-310, 1993.

[17] "Feathers and Flight," The University of Waikato, 16 September 2011. [Online]. Available: http://sciencelearn.org.nz/Contexts/Flight/Looking-Closer/Feathers-and-flight. [Accessed Febuary 2016].

[18] J. Meseguer, S. Franchini, I. Perez-Grande and J. L. Sanz, "On the Aerodynamics of Leading-edge High-lift Devices of Avian Wings," Journal of Aerospace Engineering, pp. 63-68, 2005. 
[19] "Red-Tailed Hawk," National Geographic, [Online]. Available: http://animals.nationalgeographic.com/animals/birds/red-tailed-hawk/. [Accessed 28 Febuary 2016].

[20] T. Prindle, "Preserving Bird Wings, Tails, or Talons".

[21] "Silicone Mold Making RTV Rubber," AeroMarine Products, [Online]. Available: http://www.aeromarineproducts.com/silicone-mold-making.htm\#125. [Accessed July 2015].

[22] "Aerodynamics Laboratory," West Virginia University, [Online]. Available: http://www.cemr.wvu.edu/research/center-details.php?id=50\&type=lab. [Accessed 1 March 2016].

[23] "4" Rotary Table," SHERLINE PRODUCTS, [Online]. Available: http://www.sherline.com/3700pg.htm. [Accessed 1 March 2016].

[24] "SLS-2 Structured Light 3D Scanner," DAVID, [Online]. Available: http://www.david3d.com/en/products/sls-2. [Accessed 3 March 2016].

[25] "AmScope SM-7 7X-90X Articulating Trinocular Zoom Microscope with Ring Light," AmScope, [Online]. Available: http://www.amscope.com/7x-90x-articulating-trinocularzoom-microscope-with-ring-light.html. [Accessed 3 March 2016].

[26] C. D. Griffin, P. H. Browning, S. D. Hamburg, J. A. Cox, E. E. Katzner, T. E. Katzner and W. W. Huebsch, "Free Flight Observations and Aerodynamic Analysis for BiologicallyInspired Optimization".

[27] R. H. Bonser and P. P. Purslow, "The Young's Modulus of Feather Keratin," The Journal of Experimental Biology, pp. 1029-1033, 1995.

[28] A. P. Rothmayer and W. W. Huebsch, "On the Modification of Laminar Boundary Layers Using Unsteady Surface Actuation," American Institute of Aeronautics and Astronautics, pp. 1229-1243, 2011.

[29] D. Churchill, "Birder's Eye View: Hawks, Vultures, Osprey Gather to Show off Their Soaring Skills," Bluffington Today, 10 October 2012. [Online]. Available: http://www.blufftontoday.com/accent/2012-10-10/birders-eye-view-hawks-vulturesosprey-gather-show-their-soaring-skills\#.VawHfvlmpFE. [Accessed 19 July 2015].

[30] E. Zolfagharifard, "Mystery of How the Wandering Albatross Travels 10,000 Miles in a Single Journey WITHOUT Flapping Its Wings Is Solved," 18 November 2013. [Online]. Available: http://www.dailymail.co.uk/sciencetech/article-2509211/Mystery-wanderingalbatross-travels-10-000-miles-single-journey-WITHOUT-flapping-wings-solved.html. [Accessed 19 July 2015].

[31] W. Muller and P. Giannino, "Air Transmissivity of Feathers," The Journal of Eperimental Biology, pp. 591-599, 199/8. 


\section{Appendix 1: Uncertainty Analysis}

In order to support and validate the findings of this study, an uncertainty analysis was conducted. This task was carried out using the common, addition of quadrature method. The following documentation outlines the primary formulas and information used to find the uncertainty of measured values.

Table 6: Uncertainty of Measured Values

\begin{tabular}{|c|c|c|c|c|c|}
\hline $\begin{array}{l}\text { Measured } \\
\text { Value }\end{array}$ & $\begin{array}{c}\text { Smallest } \\
\text { Increment of } \\
\text { Measurement } \\
(\Delta)\end{array}$ & Units & Uncertainty Formula & $\begin{array}{l}\text { Uncertainty } \\
\text { Calculation }\end{array}$ & $\begin{array}{l}\text { Calculated } \\
\text { Uncertainty }\end{array}$ \\
\hline $\begin{array}{l}\text { Temperature } \\
\text { (T) }\end{array}$ & \pm 1 & ${ }^{\circ} \mathrm{R}$ & $\frac{\Delta T}{T_{\text {avg. }}}(100 \%)$ & $\frac{1}{522}(100 \%)$ & $0.192 \%$ \\
\hline Pressure $(\mathrm{P})$ & \pm 0.025 & in. of $\mathrm{Hg}$ & $\frac{\Delta P}{P_{\text {avg. }}}(100 \%)$ & $\frac{0.025}{28.91}(100 \%)$ & $0.086 \%$ \\
\hline $\begin{array}{l}\text { Test Section } \\
\text { Head } \\
\text { Pressure (q) }\end{array}$ & \pm 0.0025 & in. of Water & $\frac{\Delta q}{q_{\min }}(100 \%)$ & $\frac{0.0025}{0.065}(100 \%)$ & $3.846 \%$ \\
\hline $\begin{array}{l}\text { Wing Model } \\
\text { Dimensions }\end{array}$ & $\pm \frac{1}{16}$ & in. & $\begin{array}{l}\frac{\Delta \text { Lenght }}{\text { Wing Length }}(100 \%) \\
\frac{\Delta \text { Width }}{\text { Wing Width }}(100 \%)\end{array}$ & $\begin{array}{l}{\left[\frac{\left(\frac{1}{16}\right)}{19}\right](100 \%)} \\
{\left[\frac{\left(\frac{1}{16}\right)}{11.5}\right](100 \%)}\end{array}$ & $0.329 \%$ \\
\hline $\begin{array}{c}\text { Angle of } \\
\text { Attack }(\alpha)\end{array}$ & \pm 0.01 & 。 & $\frac{\Delta \alpha}{\alpha_{\max }}(100 \%)$ & $\frac{0.01}{40}(100 \%)$ & $0.025 \%$ \\
\hline
\end{tabular}


The first steps in finding the required uncertainty values were to find the uncertainty of the measurements taken from each piece of basic equipment. The process and formulas used to complete this task are shown in Table 6. These uncertainty values were then used to find the uncertainty of calculated values throughout the study. Finding the uncertainty of the values calculated from the measured values of Table 6 can be seen in Table 7.

Table 7: Uncertainty of the Calculated Values

\begin{tabular}{|c|c|c|c|c|c|}
\hline $\begin{array}{l}\text { Calculated } \\
\text { Value }\end{array}$ & Formula & $\begin{array}{l}\text { Required } \\
\text { Measured } \\
\text { Values }\end{array}$ & $\begin{array}{l}\text { Uncertainty } \\
\text { Formula }\end{array}$ & $\begin{array}{l}\text { Uncertainty } \\
\text { Calculation }\end{array}$ & $\begin{array}{c}\text { Calculated } \\
\text { Uncertainty }\end{array}$ \\
\hline $\begin{array}{l}\text { Mean } \\
\text { Chord } \\
\text { Length of } \\
\text { the Wing } \\
\text { Models } \\
\text { (C) }\end{array}$ & $\begin{array}{c}\Sigma_{\text {Measurements }}^{\text {Chord }} \\
\text { Number of } \\
\text { Measurements }\end{array}$ & Width & $\frac{\frac{1}{16}}{\text { Width }_{\text {mean }}}$ & $\frac{\frac{1}{16}}{6.993}$ & $0.894 \%$ \\
\hline $\begin{array}{l}\text { Wing } \\
\text { Area (A) }\end{array}$ & $\sum_{\text {Measurement Interval }}^{\text {Chord Measurements }}$ & $\begin{array}{l}\text { Width, } \\
\text { Length }\end{array}$ & $\sqrt{u n c_{\text {Width }}^{2}}+u n c_{\text {Length }}^{2}$ & $\sqrt{0.329^{2}+0.543^{2}}$ & $0.635 \%$ \\
\hline $\begin{array}{l}\text { Air } \\
\text { Density } \\
(\rho)\end{array}$ & $\rho=\left(\frac{P}{R T}\right)$ & $\mathrm{P}, \mathrm{T}$ & $\sqrt{u n c_{P}^{2}+u n c_{T}^{2}}$ & $\sqrt{0.086^{2}+0.192^{2}}$ & $0.894 \%$ \\
\hline $\begin{array}{l}\text { Air } \\
\text { Velocity } \\
\text { (V) }\end{array}$ & $\begin{array}{l}\qquad \sqrt{\frac{q * 5.2 * 2}{\frac{P}{R T}}} \\
\text { Where } \mathrm{R}=\text { The Individual } \\
\text { Gas Constant for Air }\end{array}$ & $\mathrm{P}, \mathrm{T}, \mathrm{q}$ & $\sqrt{u n c_{P}^{2}+u n c_{T}^{2}+u n c_{q}^{2}}$ & $\begin{array}{c}\begin{array}{c}0.086^{2}+0.192^{2} \\
+3.846^{2}\end{array}\end{array}$ & $3.852 \%$ \\
\hline $\begin{array}{l}\text { Reynolds } \\
\text { Number } \\
\text { (Re) }\end{array}$ & $\begin{array}{l}\qquad R e=\frac{\rho V C}{\mu} \\
\text { Where } \mu=\text { The Dynamic } \\
\text { Viscosity of Air }\end{array}$ & $\mathrm{V}, \mathrm{C}, \rho$ & $\sqrt{u n c_{\rho}^{2}+u n c_{T}^{2}+u n c_{q}^{2}}$ & $\begin{array}{c}\begin{array}{c}0.894^{2}+0.192^{2} \\
+3.846^{2}\end{array}\end{array}$ & $3.960 \%$ \\
\hline
\end{tabular}


Lift and drag were found from the forces applied to the six-component balance.

Therefore, the uncertainty values for lift and drag had to be found another way. As a result, the standard error of the mean (SEM) was used.

For this approach, the values of lift and drag, recorded under the same conditions, with the same wing model were used. The mean and standard deviation were found and used in the following formula. Uncertainty of the coefficients of lift and drag were then found by the addition of quadrature method used in Table 7. The results of this process can be seen in 
Table 8.

$$
S E M=\frac{(1.96)(\sigma)}{\text { mean }}
$$

Where:

$$
\begin{aligned}
& \sigma=\text { The Standard Deviation of the Sample } \\
& \text { mean = The Mean of the Sample }
\end{aligned}
$$


Table 8: Aerodynamic Force Data Uncertainty

\begin{tabular}{|c|c|c|c|c|c|}
\hline $\begin{array}{l}\text { Calculated } \\
\text { Value }\end{array}$ & Formula & $\begin{array}{c}\text { Required } \\
\text { Measured } \\
\text { Values }\end{array}$ & $\begin{array}{c}\text { Uncertainty } \\
\text { Formula }\end{array}$ & $\begin{array}{l}\text { Uncertainty } \\
\text { Calculation }\end{array}$ & $\begin{array}{l}\text { Calculated } \\
\text { Uncertainty }\end{array}$ \\
\hline Lift (L) & N/A & N/A & $\begin{array}{l}\text { SEM } \\
=\frac{(1.96)(\sigma)}{\text { mean }}\end{array}$ & $\frac{(1.96)(0.2696)}{2.509}$ & $21.065 \%$ \\
\hline Drag (D) & N/A & N/A & $\begin{array}{l}\text { SEM } \\
=\frac{(1.96)(\sigma)}{\text { mean }}\end{array}$ & $\frac{(1.96)(.0133)}{0.1805}$ & $14.507 \%$ \\
\hline $\mathrm{L} / \mathrm{D}$ & $\frac{L}{D}$ & L, D & $\sqrt{u n c_{L}^{2}+u n c_{D}^{2}}$ & $\sqrt{21.065^{2}+14.507^{2}}$ & $26 \%$ \\
\hline $\begin{array}{l}\text { Coefficient } \\
\text { of Lift }\left(C_{L}\right)\end{array}$ & $\frac{L}{\mu A}$ & $\mathrm{~L}, \mathrm{~A}$ & $\sqrt{u n c_{L}^{2}}+u n c_{a}^{2}$ & $\sqrt{21.065^{2}+0.635^{2}}$ & $21.074 \%$ \\
\hline $\begin{array}{l}\text { Coefficient } \\
\text { of Drag }\left(\mathrm{C}_{\mathrm{d}}\right)\end{array}$ & $\frac{D}{\mu A}$ & $\mathrm{D}, \mathrm{A}$ & $\sqrt{u n c_{d}^{2}+u n c_{a}^{2}}$ & $\sqrt{14.507^{2}+0.635^{2}}$ & $14.521 \%$ \\
\hline
\end{tabular}




\section{Appendix 2: Additional Aerodynamic Force Data}

Table 9: Minimum $C_{D}$ Values for the Wing Models

\begin{tabular}{|r|c|c|c|c|c|}
\multicolumn{1}{|c}{ Re } & W1 & W2 & W3 & W4 & W5 \\
\hline 61,000 & 0.00205 & 0.00445 & 0.0135 & 0.0021 & -0.0075 \\
64,000 & 0.00688 & 0.00795 & 0.008 & 0.0108 & 0.007 \\
78,000 & 0.00175 & 0.00175 & 0.01 & 0.011 & 0.013 \\
\hline 85,000 & 0.0077 & 0.001115 & 0.032 & 0.00425 & 0.002 \\
93,000 & 0.0042 & 0.0093 & 0.01 & 0.0079 & 0.005 \\
\hline 96,000 & 0.00345 & 0.0061 & 0.009 & 0.00765 & -0.0082 \\
\hline 98,000 & 0.0043 & 0.0036 & 0.0125 & 0.0063 & -0.0014 \\
\hline 100,000 & 0.0052 & 0.007 & 0.011 & 0.0063 & 0.002 \\
\hline 110,000 & 0.0052 & 0.0053 & 0.012 & 0.00785 & 0.0065 \\
\hline
\end{tabular}

Table 10: Maximum $\mathrm{dC}_{L} / \mathrm{d} \alpha$ Values for the Wing Models

\begin{tabular}{|c|c|c|c|c|c|}
\hline Re & W1 & W2 & W3 & W4 & W5 \\
\hline 61,000 & 0.0335 & 0.0545 & 0.045 & 0.0465 & 0.044 \\
64,000 & 0.0399 & 0.062 & 0.052 & 0.053 & 0.045 \\
78,000 & 0.037 & 0.0585 & 0.0515 & 0.048 & 0.052 \\
\hline 85,000 & 0.0525 & 0.057 & 0.042 & 0.0475 & 0.04 \\
93,000 & 0.045 & 0.062 & 0.046 & 0.0475 & 0.039 \\
96,000 & 0.035 & 0.0572 & 0.046 & 0.048 & 0.0615 \\
98,000 & 0.036 & 0.053 & 0.0485 & 0.0515 & 0.0385 \\
100,000 & 0.0345 & 0.052 & 0.0485 & 0.048 & 0.041 \\
110,000 & 0.0335 & 0.052 & 0.045 & 0.046 & 0.0425 \\
\hline
\end{tabular}

Table 11: Maximum Values for the Wing Models

\begin{tabular}{|c|c|c|c|c|c|}
\hline Re & W1 & W2 & W3 & W4 & W5 \\
\hline 61,000 & 37 & 26.5 & 13.5 & 53 & 15.5 \\
\hline 64,000 & 12 & 63 & 14.4 & 17 & 200 \\
\hline 78,000 & 26 & 15 & 10.3 & 13 & 18 \\
\hline 85,000 & 35.5 & 40 & 10.27 & 38 & 15 \\
93,000 & 32 & 23.5 & 9 & 19.6 & 58 \\
\hline 96,000 & 37.5 & 32.5 & 14 & 23.5 & 115 \\
\hline 98,000 & 29 & 57 & 13 & 29.5 & 20 \\
\hline 100,000 & 15 & 23 & 12 & 25 & 70 \\
\hline 110,000 & 14.5 & 29 & 10.2 & 19.6 & 95 \\
\hline
\end{tabular}

